\title{
UNLLASSIFIED.
}

\section{SANITARY LANDFILL \\ GROUNDWATER \\ MONITORING REPORT (U)}

\section{KEY WORDS}

hazardous waste

LFW wells

radium

tritium

volatile organics

PUBLICATION DATE: AUGUST 1992

Authorized Derivative Classifier:

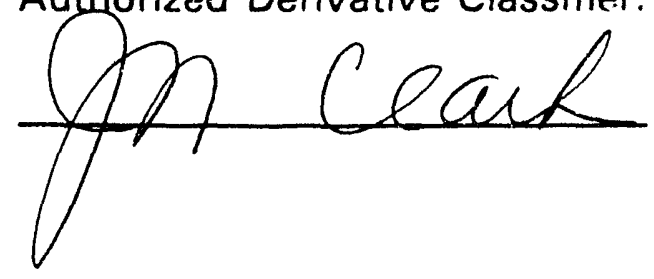




\section{SANITARY LANDFILL SECOND QUARTER 1992}

All copies of this Groundwater Quality Assessment Report transmitted to the regulatory agencies are stamped by a registered P.G. of South Carolina. Copies of the report retained at the Savannah River Site for reference do not include the registered P.G. stamp. 


\section{Second Quarter 199٪ Data Review}

This report contains analytical data for samples taken during second quarter 1992 from wells of the LFW series located at the Sanitary Landfill at the Savannah River Site. This data is submitted in reference to the Sanitary Landfill Operating Permit (DWP-087A). 
WELL LFW 6

$\begin{array}{lllllll}\text { SRS Coord. Lat/Longitude } & \text { Screen Zone Elevation } & \text { Top of Casing } & \text { Casing } & \text { Formation } \\ \text { N84537.8 } & 33.286553^{\circ} \mathrm{N} & 160.4-141.1 \mathrm{ft} \mathrm{msl} & 171.7 \mathrm{ft} \mathrm{msl} & 4^{\text {n Steel }} \\ \text { E45241.2 } & 81.711886^{\circ} \mathrm{W} & & & \end{array}$

\section{MEASUREMENTS CONDUCTED IN THE FIELD}

Sample date: 05/28/92

Depth to water: $17.59 \mathrm{ft}(5.36 \mathrm{~m})$ below TOC

Water elevation: $154.11 \mathrm{ft}(46.97 \mathrm{~m}) \mathrm{ms}$ l

Sp. conductance: $276 \mu \mathrm{S} / \mathrm{cm}$

Water evacuated before sampling: $34 \mathrm{gal}$

\section{LABORATORY ANALYSES}

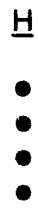

H

Analyte
pH
pH
Specific conductance
Specific conductarice
Aluminum
Aluminum
Antimony
Antimony
Arsenic
Arsenic
Barium
Barium
Benzene
Bromodichloromethane
Bromoform
Bromomethane (Methyl bromide)
Cadmium
Cadmium
Calcium
Calcium
Carbon tetrachloride
Chloride
Chlorobenzene
Chloroethane
Chloroethene (Vinyl chloride)
2-Chloroethyl vinyl ether
Chloroform
Chloromethane (Methyl chloride)
Chromium
Chromium
Copper
Copper
Dibromochloromethane
1,1 -Dichloroethane
$1,2-$ Dichloroethane
cis-1,2-Dichloroethene
1,1 -Dichloroethylene
Dichloromethane (Methylene chloride)
$2,4-$ Dichlorophenoxyacetic acid

Time: $9: 30$

$\mathrm{pH}: 5.9$

Alkalinity: $118 \mathrm{mg} / \mathrm{L}$

Water temperature: $18.0^{\circ} \mathrm{C}$

\begin{tabular}{llll} 
Result & Unit & Flag & Lab \\
\hline & & & \\
7.1 & $\mathrm{pH}$ & 0 & WA \\
7.1 & $\mathrm{pH}$ & 0 & WA \\
209 & $\mu \mathrm{S} / \mathrm{cm}$ & 0 & WA \\
209 & $\mu \mathrm{S} / \mathrm{cm}$ & 0 & WA \\
16 & $\mu \mathrm{g} / \mathrm{L}$ & 0 & WA \\
$<15$ & $\mu \mathrm{g} / \mathrm{L}$ & 0 & WA \\
8.0 & $\mu \mathrm{g} / \mathrm{L}$ & 2 & WA \\
5.5 & $\mu \mathrm{g} / \mathrm{L}$ & 2 & WA \\
5.2 & $\mu \mathrm{g} / \mathrm{L}$ & 0 & WA \\
4.9 & $\mu \mathrm{g} / \mathrm{L}$ & 0 & WA \\
12 & $\mu \mathrm{g} / \mathrm{L}$ & 0 & WA \\
11 & $\mu \mathrm{g} / \mathrm{L}$ & 0 & WA \\
1.8 & $\mu \mathrm{g} / \mathrm{L}$ & 0 & WA \\
$<5.0$ & $\mu \mathrm{g} / \mathrm{L}$ & 0 & WA \\
$<5.0$ & $\mu \mathrm{g} / \mathrm{L}$ & 0 & WA \\
$<10$ & $\mu \mathrm{g} / \mathrm{L}$ & 0 & WA \\
$<0.35$ & $\mu \mathrm{g} / \mathrm{L}$ & 0 & WA \\
1.0 & $\mu \mathrm{g} / \mathrm{L}$ & 0 & WA \\
3.790 & $\mu \mathrm{g} / \mathrm{L}$ & 0 & WA \\
3.620 & $\mu \mathrm{g} / \mathrm{L}$ & 0 & WA \\
$<5.0$ & $\mu \mathrm{g} / \mathrm{L}$ & 0 & WA \\
8.020 & $\mu \mathrm{g} / \mathrm{L}$ & 0 & WA \\
$<5.0$ & $\mu \mathrm{g} / \mathrm{L}$ & 0 & WA \\
$<10$ & $\mu \mathrm{g} / \mathrm{L}$ & 0 & WA \\
1.9 & $\mu \mathrm{g} / \mathrm{L}$ & 1 & WA \\
$<10$ & $\mu \mathrm{g} / \mathrm{L}$ & 0 & WA \\
$<5.0$ & $\mu \mathrm{g} / \mathrm{L}$ & 0 & WA \\
$<10$ & $\mu \mathrm{g} / \mathrm{L}$ & 0 & WA \\
$<1.1$ & $\mu \mathrm{g} / \mathrm{L}$ & 0 & WA \\
$<1.1$ & $\mu \mathrm{g} / \mathrm{L}$ & 0 & WA \\
$<1.1$ & $\mu \mathrm{g} / \mathrm{L}$ & 0 & WA \\
$<1.1$ & $\mu \mathrm{g} / \mathrm{L}$ & 0 & WA \\
$<5.0$ & $\mu \mathrm{g} / \mathrm{L}$ & 0 & WA \\
12 & $\mu \mathrm{g} / \mathrm{L}$ & 2 & WA \\
$<5.0$ & $\mu \mathrm{g} / \mathrm{L}$ & 0 & WA \\
$<5.0$ & $\mu \mathrm{g} / \mathrm{L}$ & 0 & WA \\
$<5.0$ & $\mu \mathrm{g} / \mathrm{L}$ & 0 & WA \\
1.6 & $\mu \mathrm{g} / \mathrm{L}$ & 0 & WA \\
$<1.1$ & $\mu \mathrm{g} / \mathrm{L}$ & 0 & WA \\
& & & \\
\hline & & 0 & 0
\end{tabular}

$\overline{- \text { exceeded holding time. }} \mathbf{a}=$ exceeded primary drinking water standard. 
WELL LFW 6 collected on 05/28/92, laboratory analyses (cont.)

H D Analyte

1,2-Dichloropropane
cis-1,3-Dichloropropene
trans-1,3-Dichloropropene
Endrin
Ethy'thenzene
Fluorile
Lead
Lead
Lindane
Magnesium
Magnesium
Manganese
Manganese
Mercury
Methoxychlor
Nickel
Nickel
Nitrate as nitrogen
Potassium
Potassium
Selenium
Selenium
Silica
Silica
Silver
Silver
Sodium
Sodium
Sulfate
1,1,2,2-Tetrachloroethane
Tetrachloroethylene
Toluene
Total organic carbon
Total organic carbon
Total organic halogens
Toxaphene
2,4,5-TP (Silvex)
1,1,1-Trichloroethane
1,1,2-Tichloroethane
Trichloroethylene
Trichlorofluoromethane
Vanadium
Vanadium
Gross alpha
Nonvolatile beta
Radium-226
Tritium

\section{Result}

$<5.0$

$<5.0$

$<5.0$

$<0.11$

$<5.0$

$<100$

$<2.0$

$<2.0$

$<0.056$

5,130

4,970

48

46

$<0.20$

$<0.56$

$<3.1$

8.1

256

1,420

1,440

$<2.0$

$<2.0$

7,650

7,470

$<0.70$

$<0.70$

4,860

4,710

5,500

$<5.0$

1.3

$<5.0$

3,840

3,840

80

$<1.1$

$<0.55$

$<5.0$

1.2

2.7

1.3

$<0.88$

$<0.88$

$4.4 E+00 \pm 1.1 E+00$

$<5.0 E+00$

1.5E + 00 $\pm 3.1 \mathrm{E}-01$

$2.3 E+00 \pm 4.1 E-01$

\begin{tabular}{|c|c|c|}
\hline Unit & Flag & $\underline{L a b}$ \\
\hline$\mu \mathrm{g} / \mathrm{L}$ & 0 & WA \\
\hline$\mu \mathrm{g} / \mathrm{L}$ & 0 & WA \\
\hline$\mu \mathrm{g} / \mathrm{L}$ & 0 & WA \\
\hline$\mu \mathrm{g} / \mathrm{L}$ & 0 & WA \\
\hline$\mu \mathrm{g} / \mathrm{L}$ & 0 & WA \\
\hline$\mu \mathrm{g} / \mathrm{L}$ & 0 & WA \\
\hline$\mu \mathrm{g} / \mathrm{L}$ & 0 & WA \\
\hline$\mu \mathrm{g} / \mathrm{L}$ & 0 & WA \\
\hline$\mu g / L$ & 0 & WA \\
\hline$\mu g / L$ & 0 & WA \\
\hline$\mu g / L$ & 0 & WA \\
\hline$\mu g / L$ & 1 & WA \\
\hline$\mu g / L$ & 1 & WA \\
\hline$\mu g / L$ & 0 & WA \\
\hline$\mu g / L$ & 0 & WA \\
\hline$\mu \mathrm{g} / \mathrm{L}$ & 0 & WA \\
\hline$\mu \mathrm{g} / \mathrm{L}$ & 0 & WA \\
\hline$\mu \mathrm{g} / \mathrm{L}$ & 0 & WA \\
\hline$\mu \mathrm{g} / \mathrm{L}$ & 0 & WA \\
\hline$\mu \mathrm{g} / \mathrm{L}$ & 0 & WA \\
\hline$\mu \mathrm{g} / \mathrm{L}$ & 0 & WA \\
\hline$\mu \mathrm{g} / \mathrm{L}$ & 0 & WA \\
\hline$\mu \mathrm{g} / \mathrm{L}$ & 0 & WA \\
\hline$\mu \mathrm{g} / \mathrm{L}$ & 0 & WA \\
\hline$\mu \mathrm{g} / \mathrm{L}$ & 0 & WA \\
\hline$\mu g / L$ & 0 & WA \\
\hline$\mu g / L$ & 0 & WA \\
\hline$\mu g / L$ & 0 & WA \\
\hline$\mu g / L$ & 0 & WA \\
\hline$\mu \mathrm{g} / \mathrm{L}$ & 0 & WA \\
\hline$\mu \mathrm{g} / \mathrm{L}$ & 0 & WA \\
\hline$\mu g / L$ & 0 & WA \\
\hline$\mu g / L$ & 0 & WA \\
\hline$\mu \mathrm{g} / \mathrm{L}$ & 0 & WA \\
\hline$\mu g / L$ & 2 & WA \\
\hline$\mu \mathrm{g} / \mathrm{L}$ & 0 & WA \\
\hline$\mu \mathrm{g} / \mathrm{L}$ & 0 & WA \\
\hline$\mu \mathrm{g} / \mathrm{L}$ & 0 & WA \\
\hline$\mu \mathrm{g} / \mathrm{L}$ & 0 & WA \\
\hline$\mu \mathrm{g} / \mathrm{L}$ & 1 & WA \\
\hline$\mu \mathrm{g} / \mathrm{L}$ & 0 & WA \\
\hline$\mu \mathrm{g} / \mathrm{L}$ & 0 & WA \\
\hline$\mu \mathrm{g} / \mathrm{L}$ & 0 & WA \\
\hline $\mathrm{pCi} / \mathrm{L}$ & 0 & $\mathrm{CN}$ \\
\hline $\mathrm{pCi} / \mathrm{L}$ & 0 & $\mathrm{CN}$ \\
\hline $\mathrm{pCi} / \mathrm{L}$ & 0 & $\mathrm{CN}$ \\
\hline $\mathrm{pCi} / \mathrm{mL}$ & 0 & $\mathrm{CN}$ \\
\hline
\end{tabular}

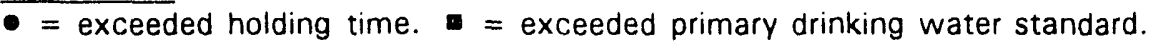


WELL LFW 7

$\begin{array}{llllll}\text { SRS Coord. } & \text { Lat/Longitude } & \text { Screen Zone Elevation } & \text { Top of Casing } & \text { Casing } & \text { Formation } \\ \text { N84310.3 } & 33.286177^{\circ} \mathrm{N} & 159.8-140.5 \mathrm{ft} \mathrm{msl} & 171.2 \mathrm{ft} \mathrm{msl} & 4 \text { "Steel } \\ \text { E45318.9 } & 81.711239^{\circ} \mathrm{W} & & & \end{array}$

MEASUREMENTS CONDUCTED IN THE FIELD

Sample date: 06/04/92

Depth to water: $19.10 \mathrm{ft}(5.82 \mathrm{~m})$ below TOC

Time: 14:00

Water elevation: $152.10 \mathrm{ft}(46.36 \mathrm{~m}) \mathrm{msl}$

Inaccessibility or pump failure prevented sample collection.

\section{WELL LFW 8}

$\begin{array}{llllll}\text { SRS Coord. } & \text { Lat/Longitude } & \text { Screen Zone Elevation } & \text { Top of Casing } & \text { Casing } & \text { Formation } \\ \text { N84032.6 } & 33.2857200^{\circ} \mathrm{N} & 159.2-139.9 \mathrm{ft} \mathrm{msl} & 170.5 \mathrm{ft} \mathrm{msl} & 4 \text { " Steel } \\ \text { E45415.3 } & 81.7104455^{\circ} \mathrm{W} & & & \end{array}$

\section{MEASUREMENTS CONDUCTED IN THE FIELD}

Sample date: 05/29/92

Depth to water: $20.65 \mathrm{ft}(6.29 \mathrm{~m})$ below TOC

W/ater elevation: $149.85 \mathrm{ft}(45.67 \mathrm{~m}) \mathrm{msl}$

Sp. conductance: $385 \mu \mathrm{S} / \mathrm{cm}$

Water evacuated before sampling: $26 \mathrm{gal}$

\section{LABORATORY ANALYSES}

Time: $10: 45$

$\mathrm{pH}: 6.6$

Alkalinity: $62 \mathrm{mg} / \mathrm{L}$

Water temperature: $18.8^{\circ} \mathrm{C}$

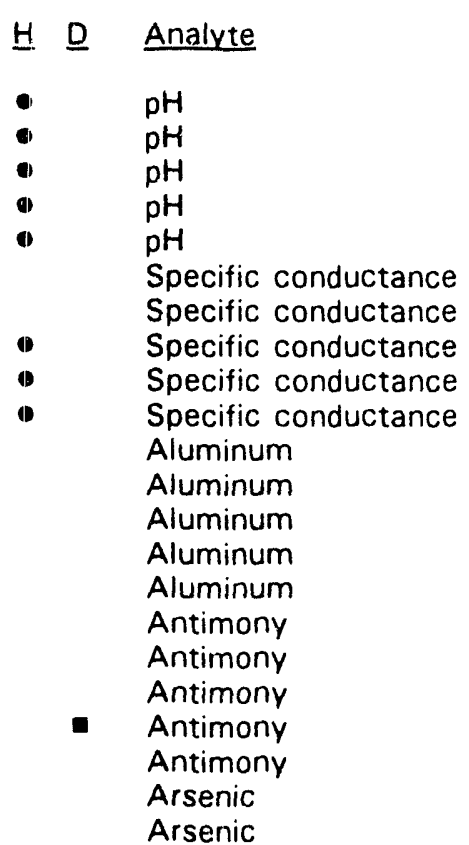

$\begin{aligned} & \text { Result } \\ & 6.3 \\ & 6.3 \\ & 6.7 \\ & 6.7 \\ & 6.1 \\ & 180 \\ & 195 \\ & 223 \\ & 224 \\ & 201 \\ &<20 \\ &<20 \\ &<20 \\ &<15 \\ &<15 \\ &<2.0 \\ &<2.0 \\ &<2.0 \\ & 9.2 \\ &<2.6 \\ & 9.4 \\ & 9.5\end{aligned}$

\begin{tabular}{llll} 
Unit & & Flag & Lab \\
\cline { 3 - 3 } $\mathrm{pH}$ & 0 & GE \\
$\mathrm{pH}$ & 0 & GE \\
$\mathrm{pH}$ & 0 & WA \\
$\mathrm{pH}$ & 0 & WA \\
$\mathrm{pH}$ & 0 & WA \\
$\mu \mathrm{S} / \mathrm{cm}$ & 0 & GE \\
$\mu \mathrm{S} / \mathrm{cm}$ & 0 & GE \\
$\mu \mathrm{S} / \mathrm{cm}$ & 0 & WA \\
$\mu \mathrm{S} / \mathrm{cm}$ & 0 & WA \\
$\mu \mathrm{S} / \mathrm{cm}$ & 0 & WA \\
$\mu \mathrm{g} / \mathrm{L}$ & 0 & GE \\
$\mu \mathrm{g} / \mathrm{L}$ & 0 & $\mathrm{GE}$ \\
$\mu \mathrm{g} / \mathrm{L}$ & 0 & $\mathrm{GE}$ \\
$\mu \mathrm{g} / \mathrm{L}$ & 0 & WA \\
$\mu \mathrm{g} / \mathrm{L}$ & 0 & WA \\
$\mu \mathrm{g} / \mathrm{L}$ & 0 & GE \\
$\mu \mathrm{g} / \mathrm{L}$ & 0 & $\mathrm{GE}$ \\
$\mu \mathrm{g} / \mathrm{L}$ & 0 & $\mathrm{GE}$ \\
$\mu \mathrm{g} / \mathrm{L}$ & 2 & WA \\
$\mu \mathrm{g} / \mathrm{L}$ & 0 & WA \\
$\mu \mathrm{g} / \mathrm{L}$ & 0 & GE \\
$\mu \mathrm{g} / \mathrm{L}$ & 0 & GE
\end{tabular}

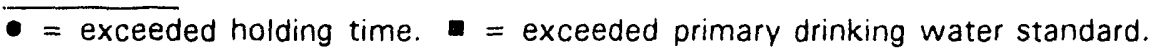


WELL LFW 8 collected on 05/29/92, laboratory analyses (cont.)

H $\underline{\text { Analyte }}$

Arsenic
Arsenic
Arsenic
Barium
Barium
Barium
Barium
Barium
Benzene
Benzene
Benzene
Benzene
Benzene
Bromodichloromethane
Bromodichloromethane
Bromodichloromethane
Bromodichloromethane
Bromodichloromethane
Bromoform
Bromoform
Bromoform
Bromoform

Bromoform

Bromomethane (Methyl bromide)

Bromomethane (Methyl bromide)

Bromomethane (Methyl bromide)

Bromomethane (Methyl bromide)

Bromomethane (Methyl bromide)

Cadmium

Cadmium

Cadmium

Cadmium

Cadmium

Calcium

Calcium

Calcium

Calcium

Calcium

Carbon tetrachloride

Carbon tetrachloride

Carbon tetrachloride

Carbon tetrachloride

Carbon tetrachloride

Chloride

Chloride

Chloride

Chloride

Chlorobenzene

Chlorobenzene

Chlorobenzene

Chlorobenzene

Chlorobenzene

Chloroethane

Chloroethane

Chloroethane

Chloroethane

\section{Result}

11

14

13

$<3.0$

$<3.0$

$<3.0$

7.0

4.3

2.6

2.7

2.7

2.8

$<5.0$

$<1.0$

$<1.0$

$<1.0$

$<5.0$

$<5.0$

$<1.0$

$<1.0$

$<1.0$

$<5.0$

$<5.0$

$<1.0$

$<1.0$

$<1.0$

$<10$

$<10$

$<2.0$

$<2.0$

$<2.0$

0.50

0.65

7,460

7,540

7,540

6,820

6,900

$<1.0$

$<1.0$

$<1.0$

$<5.0$

$<5.0$

16,700

16,800

15,900

17,500

13

13

13

11

15

7.0

7.0

8.5

$<10$

\begin{tabular}{|c|c|c|}
\hline Unit & Flag & La \\
\hline$\mu g / \mathrm{L}$ & 0 & $\mathrm{GE}$ \\
\hline$\mu \mathrm{g} / \mathrm{L}$ & 0 & $W$ \\
\hline$\mu \mathrm{g} / \mathrm{L}$ & 0 & $w$ \\
\hline$\mu \mathrm{g} / \mathrm{L}$ & 0 & $\mathrm{GE}$ \\
\hline$\mu \mathrm{g} / \mathrm{L}$ & 0 & $\mathrm{G}$ \\
\hline$\mu g / L$ & 0 & $\mathrm{GE}$ \\
\hline$\mu \mathrm{g} / \mathrm{L}$ & 0 & $W$ \\
\hline$\mu \mathrm{g} / \mathrm{L}$ & 0 & W \\
\hline$\mu \mathrm{g} / \mathrm{L}$ & 1 & G \\
\hline$\mu g / L$ & 1 & $\mathbf{G}$ \\
\hline$\mu \mathrm{g} / \mathrm{L}$ & 1 & $\mathbf{G}$ \\
\hline$\mu g / L$ & 1 & $W$ \\
\hline$\mu \mathrm{g} / \mathrm{L}$ & 0 & $w$ \\
\hline$\mu \mathrm{g} / \mathrm{L}$ & 0 & $\mathbf{G}$ \\
\hline$\mu \mathrm{g} / \mathrm{L}$ & 0 & $\mathrm{G}$ \\
\hline$\mu \mathrm{g} / \mathrm{L}$ & 0 & $\mathbf{G}$ \\
\hline$\mu \mathrm{g} / \mathrm{L}$ & 0 & W \\
\hline$\mu \mathrm{g} / \mathrm{L}$ & 0 & $w$ \\
\hline$\mu g / L$ & 0 & $\mathbf{G}$ \\
\hline$\mu \mathrm{g} / \mathrm{L}$ & 0 & $\bar{G}$ \\
\hline$\mu \mathrm{g} / \mathrm{L}$ & 0 & $\mathbf{G}$ \\
\hline$\mu \mathrm{g} / \mathrm{L}$ & 0 & $w$ \\
\hline$\mu \mathrm{g} / \mathrm{L}$ & 0 & W \\
\hline$\mu \mathrm{g} / \mathrm{L}$ & 0 & G \\
\hline$\mu g / L$ & 0 & G \\
\hline$\mu \mathrm{g} / \mathrm{L}$ & 0 & G \\
\hline$\mu \mathrm{g} / \mathrm{L}$ & 0 & $u$ \\
\hline$\mu \mathrm{g} / \mathrm{L}$ & 0 & \\
\hline$\mu \mathrm{g} / \mathrm{L}$ & 0 & G \\
\hline$\mu \mathrm{g} / \mathrm{L}$ & 0 & $\mathrm{G}$ \\
\hline$\mu \mathrm{g} / \mathrm{L}$ & 0 & G \\
\hline$\mu \mathrm{g} / \mathrm{L}$ & 0 & n \\
\hline$\mu \mathrm{g} / \mathrm{L}$ & 0 & \\
\hline$\mu \mathrm{g} / \mathrm{L}$ & 0 & G \\
\hline$\mu \mathrm{g} / \mathrm{L}$ & 0 & G \\
\hline$\mu \mathrm{g} / \mathrm{L}$ & 0 & G \\
\hline$\mu \mathrm{g} / \mathrm{L}$ & 0 & n \\
\hline$\mu \mathrm{g} / \mathrm{L}$ & 0 & \\
\hline$\mu \mathrm{g} / \mathrm{L}$ & 0 & G \\
\hline$\mu \mathrm{g} / \mathrm{L}$ & 0 & G \\
\hline$\mu \mathrm{g} / \mathrm{L}$ & 0 & $\mathrm{G}$ \\
\hline$\mu \mathrm{g} / \mathrm{L}$ & 0 & n \\
\hline$\mu \mathrm{g} / \mathrm{L}$ & 0 & \\
\hline$\mu \mathrm{g} / \mathrm{L}$ & 0 & G \\
\hline$\mu \mathrm{g} / \mathrm{L}$ & 0 & G \\
\hline$\mu \mathrm{g} / \mathrm{L}$ & 0 & u \\
\hline$\mu g / L$ & 0 & u \\
\hline$\mu \mathrm{g} / \mathrm{L}$ & 2 & G \\
\hline$\mu \mathrm{g} / \mathrm{L}$ & 2 & G \\
\hline$\mu \mathrm{g} / \mathrm{L}$ & 2 & G \\
\hline$\mu \mathrm{g} / \mathrm{L}$ & 2 & n \\
\hline$\mu \mathrm{g} / \mathrm{L}$ & 2 & n \\
\hline$\mu \mathrm{g} / \mathrm{L}$ & 1 & G \\
\hline$\mu g / L$ & $i$ & G \\
\hline$\mu \mathrm{g} / \mathrm{L}$ & $i$ & G \\
\hline & & \\
\hline
\end{tabular}

C

- =xceeded holding time. = exceeded primary drinking water standard. 
WELL LFW 8 collected on 05/29/92, laboratory analyses (cont.)

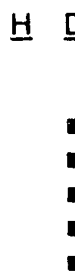

Chloroethane

- Chloroethene (Vinyl chloride)

- Chloroethene (Vinyl chloride)

- Chloroethene (Vinyl chloride)

- Chloroethene (Vinyl chloride)

Chloroethene (Vinyl chloride)

2-Chloroethyl vinyl ether

2-Chloroethyl vinyl ether

2-Chloroethyl vinyl ether

2-Chloroethyl vinyl ether

2-Chloroethyl vinyl ether

Chloroform

Chloroform

Chloroform

Chloroform

Chloroform

Chloromethane (Methyl chloride)

Chloromethane (Methyl chloride)

Chloromethane (Methyl chloride)

Chloromethane (Methyl chloride)

Chloromethane (Methyl chloride)

Chromium

Chromium

Chromium

Chromium

Chromium

Copper

Copper

Copper

Copper

Copper

Dibromochloromethane

Dibromochloromethane

Dibromochloromethane

Dibromochloromethane

Dibromochloromethane

1,1-Dichloroethane

1,1-Dichloroethane

1,1-Dichloroethane

1,1-Dichloroethane

1,1-Dichloroethane

1,2-Dichloroethane

1,2-Dichloroethane

1,2-Dichloroethane

1,2-Dichloroethane

1,2-Dichloroethane

cis-1,2-Dichloroethene

cis-1,2-Dichloroethene

1,1-Dichloroethylene

1,1-Dichloroethylene

1,1-Dichloroethylene

1,1-Dichloroethylene

1,1-Dichloroethylene

trans-1,2-Dichloroethylene

trans-1,2-Dichloroethylene

trans-1,2-Dichloroethylene

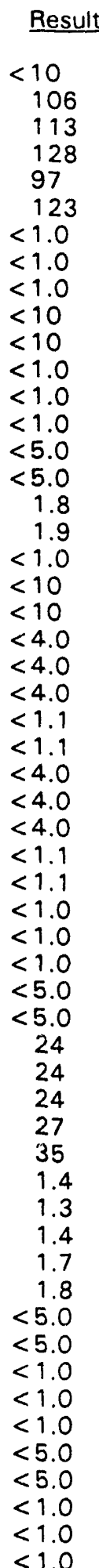

Result

Unit Flag Lab

$\mu g / L \quad 0 \quad$ WA

$\mu \mathrm{g} / \mathrm{L} \quad 2 \quad \mathrm{GE}$

$\mu g / L \quad 2 \quad$ GE

$\mu \mathrm{g} / \mathrm{L} \quad 2 \quad \mathrm{GE}$

$\mu g / L \quad 2 \quad$ WA

$\mu \mathrm{g} / \mathrm{L} \quad 2 \quad$ WA

$\mu g / L \quad 0 \quad$ GE

$\mu \mathrm{g} / \mathrm{L} \quad 0 \quad \mathrm{GE}$

$\mu g / L \quad 0 \quad$ GE

$\mu \mathrm{g} / \mathrm{L} \quad \mathrm{O} \quad$ WA

$\mu g / L \quad$ WA

$\mu \mathrm{g} / \mathrm{L} \quad 0 \quad \mathrm{GE}$

$\mu g / L \quad 0 \quad G E$

$\mu \mathrm{g} / \mathrm{L} \quad \mathrm{O} \quad \mathrm{GE}$

$\mu g / L \quad O \quad W A$

$\mu \mathrm{g} / \mathrm{L} \quad 0 \quad$ WA

$\mu g / L \quad 0 \quad$ GE

$\mu \mathrm{g} / \mathrm{L} \quad \mathrm{O} \quad \mathrm{GE}$

$\mu \mathrm{g} / \mathrm{L} \quad 0 \quad \mathrm{GE}$

$\mu \mathrm{g} / \mathrm{L} \quad \mathrm{O} \quad$ WA

$\mu g / L \quad O \quad W A$

$\mu g / L \quad 0 \quad$ GE

$\mu g / L \quad O \quad G E$

$\mu g / L \quad 0 \quad$ GE

$\mu g / L \quad O \quad W A$

$\mu g / L \quad 0 \quad$ WA

$\mu g / L \quad O \quad$ GE

$\mu g / L \quad O \quad$ GE

$\mu \mathrm{g} / \mathrm{L} \quad \mathrm{O} \quad \mathrm{GE}$

$\mu g / L \quad O \quad$ WA

$\mu g / L \quad O \quad W A$

$\mu g / L \quad 0 \quad$ GE

$\mu g / L \quad O \quad G E$

$\mu \mathrm{g} / \mathrm{L} \quad 0 \quad \mathrm{GE}$

$\mu g / L \quad O \quad W A$

$\mu \mathrm{g} / \mathrm{L} \quad 0 \quad$ WA

$\mu g / L \quad 2 \quad$ GE

$\mu \mathrm{g} / \mathrm{L} \quad 2 \quad \mathrm{GE}$

$\mu g / L \quad 2 \quad$ GE

$\mu g / L \quad 2 \quad W A$

$\mu g / L \quad 2 \quad$ WA

$\mu \mathrm{g} / \mathrm{L} \quad 0 \quad \mathrm{GE}$

$\mu g / L \quad 0 \quad G E$

$\mu \mathrm{g} / \mathrm{L} \quad \mathrm{O} \quad \mathrm{GE}$

$\mu \mathrm{g} / \mathrm{L} \quad 0 \quad$ WA

$\mu g / L \quad 0 \quad$ WA

$\mu g / L \quad 0 \quad$ WA

$\mu g / L \quad O \quad$ WA

$\mu g / L \quad O \quad G E$

$\mu \mathrm{g} / \mathrm{L} \quad \mathrm{O} \quad \mathrm{GE}$

$\mu g / L \quad O \quad G E$

$\mu g / L \quad 0 \quad W A$

$\mu g / L \quad 0 \quad$ WA

$\mu \mathrm{g} / \mathrm{L} \quad \mathrm{O} \quad \mathrm{GE}$

$\mu \mathrm{g} / \mathrm{L} \quad \mathrm{O} \quad \mathrm{GE}$

$\mu \mathrm{g} / \mathrm{L}$

$\mathrm{GE}$

- exceeded holding time. = exceeded primary drinking water standard. 
WELL LFW 8 collected on 05/29/92, laboratory analyses (cont.)

H D Analyte

Dichloromethane (Methylene chloride)

Dichloromethane (Methylene chloride)

Dichloromethane (Methylene chloride)

Dichloromethane (Methylene chloride)

Dichloromethane (Methylene chloride)

2,4-Dichlorophenoxyacetic acid

2,4-Dichlorophenoxyacetic acid

2,4-Dichlorophenoxyacetic acid

2,4-Dichlorophenoxyacetic acid

1,2-Dichloropropane

1,2-Dichloropropane

1,2-Dichloropropane

1,2-Dichloropropane

1,2-Dichloropropane

cis-1,3-Dichloropropene

cis-1,3-Dichloropropene

cis-1,3-Dichloropropene

cis-1,3-Dichloropropene

cis-1,3-Dichloropropene

trans-1,3-Dichloropropene

trans-1,3-Dichloropropene

trans-1,3-Dichloropropene

trans-1,3-Dichloropropene

trans-1,3-Dichloropropene

Endrin

Endrin

Endrin

Endrin

Endrin

Ethylbenzene

Ethylbenzene

Ethylbenzene

Ethylbenzene

Ethylbenzene

Fluoride

Fluoride

Fluoride

Fluoride

Lead

Lead

Lead

Lead

Lead

Lindane

Lindane

Lindane

Lindane

Lindane

Magnesium

Magnesium

Magnesium

Magnesium

Magnesium

Manganese

Manganese

Manganese

\section{Result}

\section{5}

5.2

3.8

3.9

5.9

$<0.30$

$<0.30$

$<1.1$

$<1.1$

$<1.0$

$<1.0$

$<1.0$

$<5.0$

$<5.0$

$<1.0$

$<1.0$

$<1.0$

$<5.0$

$<5.0$

$<1.0$

$<1.0$

$<1.0$

$<5.0$

$<5.0$

$<0.0060$

$<0.0060$

$<0.11$

$<0.22$

$<0.11$

13

13

13

11

16

200

197

175

165

$<3.0$

$<3.0$

$<3.0$

$<2.0$

$<2.0$

$<0.0050$

$<0.0050$

$<0.056$

$<0.11$

$<0.056$

11,800

12,100

11,900

11,000

11,000

26

26

26
Unit $\quad \underline{\text { Flag }} \quad \underline{\text { Lab }}$

$\mu \mathrm{g} / \mathrm{L}$

$\mu \mathrm{g} / \mathrm{L}$

$\mu \mathrm{g} / \mathrm{L}$

$\mu \mathrm{g} / \mathrm{L}$

$\mu \mathrm{g} / \mathrm{L}$

$\mu g / L$

$\mu \mathrm{g} / \mathrm{L}$

$\mu \mathrm{g} / \mathrm{L}$

$\mu g / L$

$\mu \mathrm{g} / \mathrm{L}$

$\mu \mathrm{g} / \mathrm{L}$

$\mu g / L$

$\mu \mathrm{g} / \mathrm{L}$

$\mu \mathrm{g} / \mathrm{L}$

$\mu \mathrm{g} / \mathrm{L}$

$\mu g / L$

$\mu \mathrm{g} / \mathrm{L}$

$\mu \mathrm{g} / \mathrm{L}$

$\mu \mathrm{g} / \mathrm{L}$

$\mu \mathrm{g} / \mathrm{L}$

$\mu \mathrm{g} / \mathrm{L}$

$\mu g / L$

$\mu \mathrm{g} / \mathrm{L}$

$\mu g^{\prime} / L$

$\mu \mathrm{g} / \mathrm{L}$

$\mu \mathrm{g} / \mathrm{L}$

$\mu \mathrm{g} / \mathrm{L}$

$\mu \mathrm{g} / \mathrm{L}$

$\mu \mathrm{g} / \mathrm{L}$

$\mu g / L$

$\mu g / L$

$\mu g / L$

$\mu g / L$

$\mu g / L$

$\mu \mathrm{g} / \mathrm{L}$

$\mu g / L$

$\mu g / L$

$\mu \mathrm{g} / \mathrm{L}$

$\mu \mathrm{g} / \mathrm{L}$

$\mu \mathrm{g} / \mathrm{L}$

$\mu g / L$

$\mu \mathrm{g} / \mathrm{L}$

$\mu \mathrm{g} / \mathrm{L}$

$\mu \mathrm{g} / \mathrm{L}$

$\mu \mathrm{g} / \mathrm{L}$

$\mu g / L$

$\mu \mathrm{g} / \mathrm{L}$

$\mu \mathrm{g} / \mathrm{L}$

$\mu \mathrm{g} / \mathrm{L}$

$\mu \mathrm{g} / \mathrm{L}$

$\mu \mathrm{g} / \mathrm{L}$

$\mu \mathrm{g} / \mathrm{L}$

$\mu \mathrm{g} / \mathrm{L}$

$\mu \mathrm{g} / \mathrm{L}$

$\mu \mathrm{g} / \mathrm{L}$

$\mu \mathrm{g} / \mathrm{L}$
$0 \quad \mathrm{GE}$

GE

GE

WA

WA

GE

GE

WA

WA

GE

GE

GE

WA

WA

GE

GE

GE

WA

WA

GE

GE

GE

WA

WA

GE

GE

WA

WA

WA

GE

GE

GE

WA

WA

GE

GE

WA

WA

GE

GE

GE

WA

WA

GE

$\mathrm{GE}$

WA

WA

WA

GE

GE

GE

WA

WA

GE

$\mathrm{GE}$

GE

- =xceeded holding time. $=$ exceeded primary drinking water standard. 
WELL LFW 8 collected on 05/29/92, laboratory analyses (cont.)

H $\underline{\text { Analyte }}$

Manganese
Manganese
Mercury
Mercury
Mercury
Mercury
Mercury
Methoxychlor
Methoxychlor
Methoxychlor
Methoxychlor
Methoxychlor
Methoxychlor
Nickel
Nickel
Nickel
Nickel
Nickel

Nitrate as nitrogen

Nitrate as nitrogen

Nitrate as nitrogen

Nitrate as nitrogen

Potassium

Potassium

Potassium

Potassium

Potassium

Selenium

Selenium

Selenium

Selenium

Selenium

Silica

Silica

Silica

Silica

Silica

Silver

Silver

Silver

Silver

Silver

Sodium

Sodium

Sodium

Sodium

Sodium

Sulfate

Sulfate

Sulfate

Sulfate

$1,1,2,2$-Tetrachloroethane

$1,1,2,2$-Tetrachloroethane

$1,1,2,2$-Tetrachloroethane

$1,1,2,2$-Tetrachloroethane

1,1,2,2-Tetrachloroethane

\section{Result}

21

20

$<0.20$

$<0.20$

$<0.20$

$<0.20$

$<0.20$

$<0.50$

$<0.50$

$<0.56$

$<1.1$

$<1.1$

$<0.56$

$<4.0$

$<4.0$

$<4.0$

$<3.1$

$<3.1$

70

80

$<10$

210

1,700

1,670

1,720

1,270

1,440

$<2.0$

$<2.0$

$<2.0$

$<2.0$

$<2.0$

5.720

5,680

5,640

5,050

5,000

$<2.0$

$<2.0$

$<2.0$

$<0.70$

$<0.70$

16,100

16,200

16,000

16,100

15,700

$<1,000$

$<1,000$

535

$<2,500$

$<1.0$

$<1.0$

$<1.0$

$<5.0$

$<5.0$

\begin{tabular}{|c|c|c|}
\hline Unit & Flag & $\underline{L a b}$ \\
\hline$\mu \mathrm{g} / \mathrm{L}$ & 0 & WA \\
\hline$\mu g / L$ & 0 & WA \\
\hline$\mu g / L$ & 0 & GE \\
\hline$\mu \mathrm{g} / \mathrm{L}$ & 0 & GE \\
\hline$\mu g / L$ & 0 & WA \\
\hline$\mu \mathrm{g} / \mathrm{L}$ & 0 & WA \\
\hline$\mu \mathrm{g} / \mathrm{L}$ & 0 & WA \\
\hline$\mu \mathrm{g} / \mathrm{L}$ & 0 & GE \\
\hline$\mu g / L$ & 0 & GE \\
\hline$\mu g / L$ & 0 & WA \\
\hline$\mu \mathrm{g} / \mathrm{L}$ & 0 & WA \\
\hline$\mu \mathrm{g} / \mathrm{L}$ & 0 & WA \\
\hline$\mu g / L$ & 0 & WA \\
\hline$\mu g / L$ & 0 & GE \\
\hline$\mu \mathrm{g} / \mathrm{L}$ & 0 & GE \\
\hline$\mu \mathrm{g} / \mathrm{L}$ & 0 & GE \\
\hline$\mu \mathrm{g} / \mathrm{L}$ & 0 & WA \\
\hline$\mu \mathrm{g} / \mathrm{L}$ & 0 & WA \\
\hline$\mu \mathrm{g} / \mathrm{L}$ & 0 & GE \\
\hline$\mu \mathrm{g} / \mathrm{L}$ & 0 & GE \\
\hline$\mu \mathrm{g} / \mathrm{L}$ & 0 & WA \\
\hline$\mu g / L$ & 0 & WA \\
\hline$\mu g / L$ & 0 & GE \\
\hline$\mu \mathrm{g} / \mathrm{L}$ & 0 & GE \\
\hline$\mu \mathrm{g} / \mathrm{L}$ & 0 & GE \\
\hline$\mu \mathrm{g} / \mathrm{L}$ & 0 & WA \\
\hline$\mu g / L$ & 0 & WA \\
\hline$\mu \mathrm{g} / \mathrm{L}$ & 0 & GE \\
\hline$\mu \mathrm{g} / \mathrm{L}$ & 0 & GE \\
\hline$\mu \mathrm{g} / \mathrm{L}$ & 0 & GE \\
\hline$\mu g / L$ & 0 & WA \\
\hline$\mu \mathrm{g} / \mathrm{L}$ & 0 & WA \\
\hline$\mu \mathrm{g} / \mathrm{L}$ & 0 & GE \\
\hline$\mu \mathrm{g} / \mathrm{L}$ & 0 & GE \\
\hline$\mu \mathrm{g} / \mathrm{L}$ & 0 & GE \\
\hline$\mu \mathrm{g} / \mathrm{L}$ & 0 & WA \\
\hline$\mu \mathrm{g} / \mathrm{L}$ & 0 & WA \\
\hline$\mu \mathrm{g} / \mathrm{L}$ & 0 & GE \\
\hline$\mu \mathrm{g} / \mathrm{L}$ & 0 & $\mathrm{GE}$ \\
\hline$\mu \mathrm{g} / \mathrm{L}$ & 0 & $\mathrm{GE}$ \\
\hline$\mu g / L$ & 0 & WA \\
\hline$\mu \mathrm{g} / \mathrm{L}$ & 0 & WA \\
\hline$\mu g / L$ & 0 & GE \\
\hline$\mu \mathrm{g} / \mathrm{L}$ & 0 & GE \\
\hline$\mu \mathrm{g} / \mathrm{L}$ & 0 & GE \\
\hline$\mu \mathrm{g} / \mathrm{L}$ & 0 & WA \\
\hline$\mu \mathrm{g} / \mathrm{L}$ & 0 & WA \\
\hline$\mu \mathrm{g} / \mathrm{L}$ & 0 & GE \\
\hline$\mu \mathrm{g} / \mathrm{L}$ & 0 & GE \\
\hline$\mu \mathrm{g} / \mathrm{L}$ & 0 & WA \\
\hline$\mu \mathrm{g} / \mathrm{L}$ & 0 & WA \\
\hline$\mu g / L$ & 0 & GE \\
\hline$\mu g / L$ & 0 & GE \\
\hline$\mu \mathrm{g} / \mathrm{L}$ & 0 & $\mathrm{GE}$ \\
\hline$\mu \mathrm{g} / \mathrm{L}$ & 0 & WA \\
\hline$\mu \mathrm{g} / \mathrm{L}$ & 0 & WA \\
\hline
\end{tabular}

- = exceeded holding time.

$\omega=$ exceeded primary drinking water standard. 
WELL LFW 8 collected on 05/29/92, laboratory analyses (cont.)

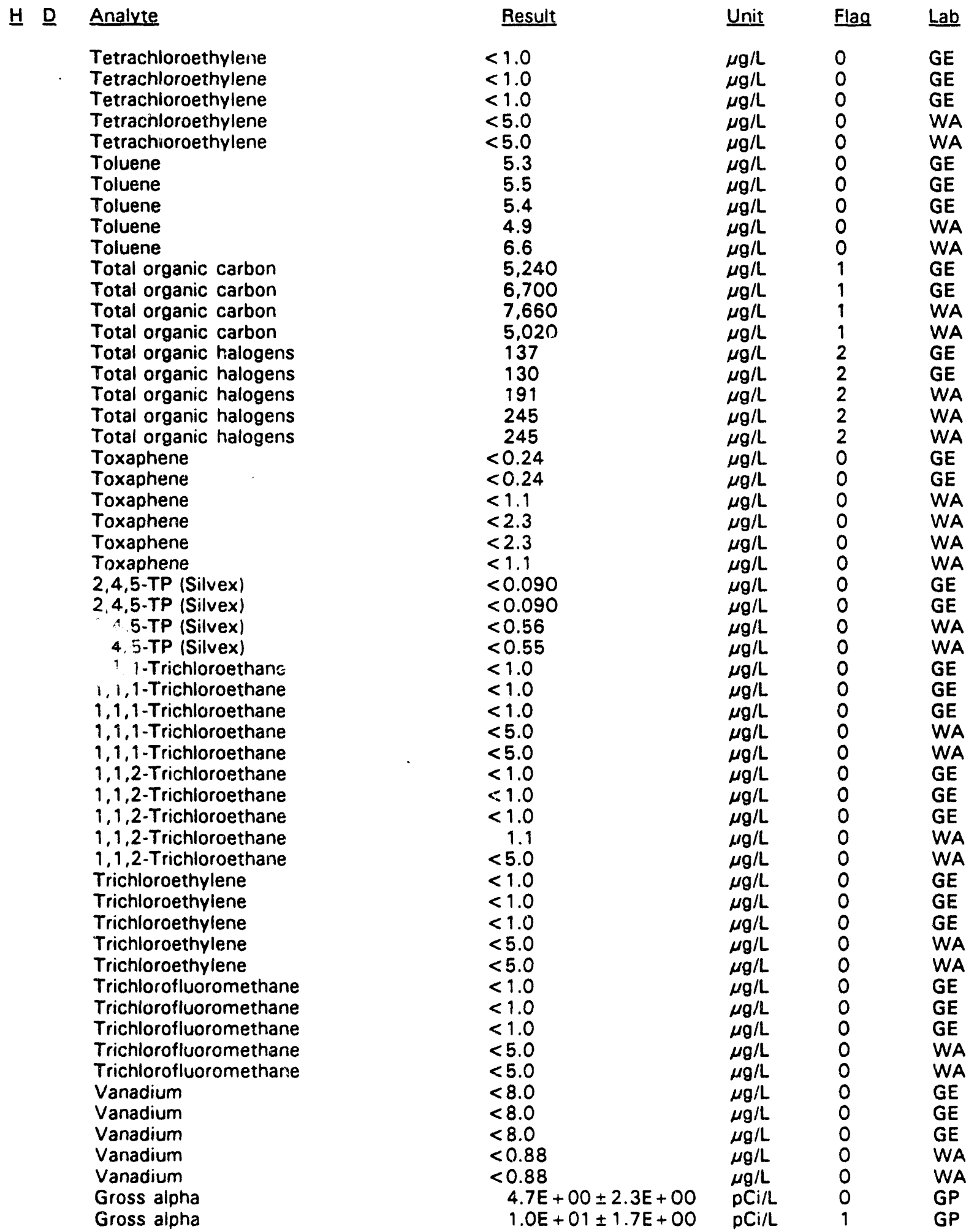

$\overline{0}$ = exceeded holding time. $=$ exceeded primary drinking water standard. 
WELL LFW 8 collected on 05/29/92, laboratory analyses (cont.)

H. Analyte
Gross alpha
Gross alpha
Nonvolatile beta
Nonvolatile beta
Nonvolatile beta
Nonvolatile beta
Radium-226
Radium-226
Radium-226
Radium-226
Tritium
Tritium
Tritium
Tritium
Tritium

$\begin{array}{llll}\text { Result } & \text { Unit } & \text { Flag } & \text { Lab } \\ 6.4 \mathrm{E}+00 \pm 6.5 \mathrm{E}-01 & \mathrm{pCi} / \mathrm{L} & 0 & \mathrm{CN} \\ 4.9 \mathrm{E}+00 \pm 5.0 \mathrm{E}-01 & \mathrm{pCi} / \mathrm{L} & 0 & \mathrm{CN} \\ 1.0 \mathrm{E}+01 \pm 2.3 \mathrm{E}+00 & \mathrm{pCi} / \mathrm{L} & 0 & \mathrm{GP} \\ 1.1 \mathrm{E}+01 \pm 1.7 \mathrm{E}+00 & \mathrm{pCi} / \mathrm{L} & 0 & \mathrm{GP} \\ 1.1 \mathrm{E}+01 \pm 1.3 \mathrm{E}+00 & \mathrm{pCi} / \mathrm{L} & 0 & \mathrm{CN} \\ 1.2 \mathrm{E}+01 \pm 1.2 \mathrm{E}+00 & \mathrm{pCi} / \mathrm{L} & 0 & \mathrm{CN} \\ 2.5 \mathrm{E}+00 \pm 3.0 \mathrm{E}-01 & \mathrm{pCi} / \mathrm{L} & 0 & \mathrm{GP} \\ <5.0 \mathrm{E}-01 & \mathrm{pCi} / \mathrm{L} & 0 & \mathrm{GP} \\ 1.6 \mathrm{E}+00 \pm 3.2 \mathrm{E}-01 & \mathrm{pCi} / \mathrm{L} & 0 & \mathrm{CN} \\ 2.3 \mathrm{E}+00 \pm 3.5 \mathrm{E}-01 & \mathrm{pCi} / \mathrm{L} & 0 & \mathrm{CN} \\ 6.5 \mathrm{E}+00 \pm 5.0 \mathrm{E}-01 & \mathrm{pCi} / \mathrm{mL} & 0 & \mathrm{GP} \\ 7.1 \mathrm{E}+00 \pm 5.0 \mathrm{E}-01 & \mathrm{pCi} / \mathrm{mL} & 0 & \mathrm{GP} \\ 7.3 \mathrm{E}+00 \pm 5.0 \mathrm{E}-01 & \mathrm{pCi} / \mathrm{mL} & 0 & \mathrm{GP} \\ 5.8 \mathrm{E}+00 \pm 5.2 \mathrm{E}-01 & \mathrm{pCi} / \mathrm{mL} & 0 & \mathrm{CN} \\ 5.9 \mathrm{E}+00 \pm 5.3 \mathrm{E}-01 & \mathrm{pCi} / \mathrm{mL} & 0 & \mathrm{CN}\end{array}$

\section{WELL LFW 10A}

$\begin{array}{llllll}\text { SRS Coord. Lat/Longitude } & \text { Screen Zone Elevation } & \text { Top of Casing } & \text { Casing } & \text { Formation } \\ \text { N84369.6 } & 33.287315^{\circ} \mathrm{N} & 159.2-129.2 \mathrm{ft} \mathrm{msl} & & 175.5 \mathrm{ft} \mathrm{msl} & \text { 4" PVC } \\ \text { E45935.6 } & 81.709731^{\circ} \mathrm{W} & & & \end{array}$

\section{MEASUREMENTS CONDUCTED IN THE FIELD}

Sample date: 05/29/92

Depth to water: $23.11 \mathrm{ft}(7.04 \mathrm{~m})$ below TOC

Water elevation: $152.39 \mathrm{ft}(46.45 \mathrm{~m}) \mathrm{msl}$

Sp. conductance: $692 \mu \mathrm{S} / \mathrm{cm}$

Water evacuated before sampling: $61 \mathrm{gal}$

\section{LABORATORY ANALYSES}

Time: 10:00

pH: 6.3

Alkalinity: $267 \mathrm{mg} / \mathrm{L}$

Water temperature: $19.1^{\circ} \mathrm{C}$

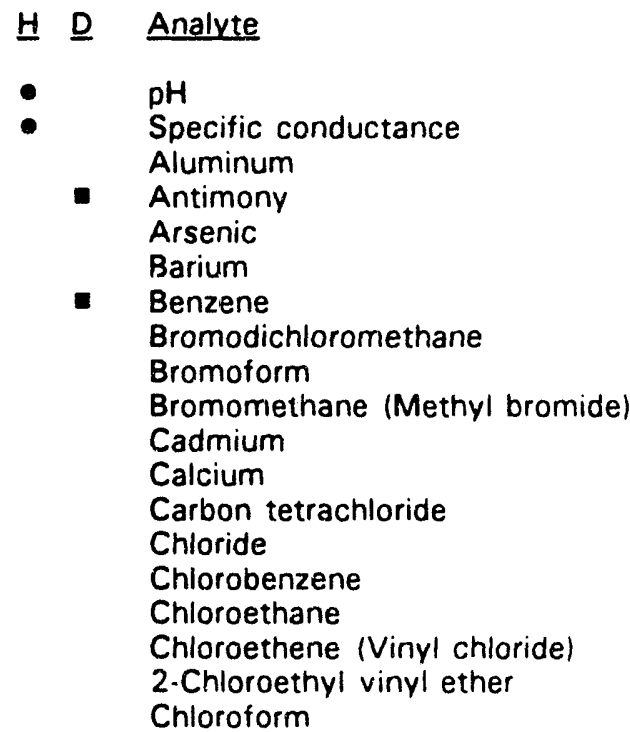

H $\underline{\text { Analyte }}$

Chloroform

$\begin{aligned} & \text { Result } \\ & 6.2 \\ & 372 \\ &< 15 \\ & 8.3 \\ & 9.0 \\ & 9.0 \\ & 8.6 \\ &< 5.0 \\ &< 5.0 \\ &< 10 \\ & 1.1 \\ & 11,400 \\ &< 5.0 \\ & 31,900 \\ &< 5.0 \\ &< 10 \\ &< 10 \\ &< 10 \\ &<5.0\end{aligned}$

\begin{tabular}{lll} 
Unit & Flag & Lab \\
\hline $\mathrm{pH}$ & 0 & WA \\
$\mu \mathrm{S} / \mathrm{cm}$ & 1 & WA \\
$\mu \mathrm{g} / \mathrm{L}$ & 0 & WA \\
$\mu \mathrm{g} / \mathrm{L}$ & 2 & WA \\
$\mu \mathrm{g} / \mathrm{L}$ & 0 & WA \\
$\mu \mathrm{g} / \mathrm{L}$ & 0 & WA \\
$\mu \mathrm{g} / \mathrm{L}$ & 2 & WA \\
$\mu \mathrm{g} / \mathrm{L}$ & 0 & WA \\
$\mu \mathrm{g} / \mathrm{L}$ & 0 & WA \\
$\mu \mathrm{g} / \mathrm{L}$ & 0 & WA \\
$\mu \mathrm{g} / \mathrm{L}$ & 0 & WA \\
$\mu \mathrm{g} / \mathrm{L}$ & 0 & WA \\
$\mu \mathrm{g} / \mathrm{L}$ & 0 & WA \\
$\mu \mathrm{g} / \mathrm{L}$ & 0 & WA \\
$\mu \mathrm{g} / \mathrm{L}$ & 0 & WA \\
$\mu \mathrm{g} / \mathrm{L}$ & 0 & WA \\
$\mu \mathrm{g} / \mathrm{L}$ & 0 & WA \\
$\mu \mathrm{g} / \mathrm{L}$ & 0 & WA \\
$\mu \mathrm{g} / \mathrm{L}$ & 0 & WA
\end{tabular}

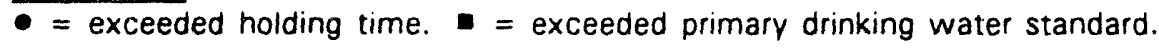


WELL LFW 1OA collected on 05/29/92, laboratory analyses (cont.)



$\overline{-}=$ exceeded holding time. $=$ exceeded primary drinking water standard. 
WELL LFW 16

$\begin{array}{llllll}\text { SRS Coord. } & \text { Lat/Longitude } & \text { Screen Zone Elevation } & \text { Top of Casing } & \text { Casing } & \text { Formation } \\ \text { N84748.9 } & 33.288018^{\circ} \mathrm{N} & 161.2-131.2 \mathrm{ft} \mathrm{msl} & 178.8 \mathrm{ft} \mathrm{msl} & 4^{\prime \prime} \mathrm{PVC} & \\ \text { E45852.6 } & 81.710687^{\circ} \mathrm{W} & & & \end{array}$

\section{MEASUREMENTS CONDUCTED IN THE FIELD}

Sample date: $05 / 28 / 92$

Depth to water: $23.43 \mathrm{ft}(7.14 \mathrm{~m})$ below TOC

Water elevation: $155.37 \mathrm{ft}(47.36 \mathrm{~m}) \mathrm{msl}$

Sp. conductance: $41 \mu \mathrm{S} / \mathrm{cm}$

Water evacuated before sampling: $63 \mathrm{gal}$

\section{LABORATORY ANALYSES}

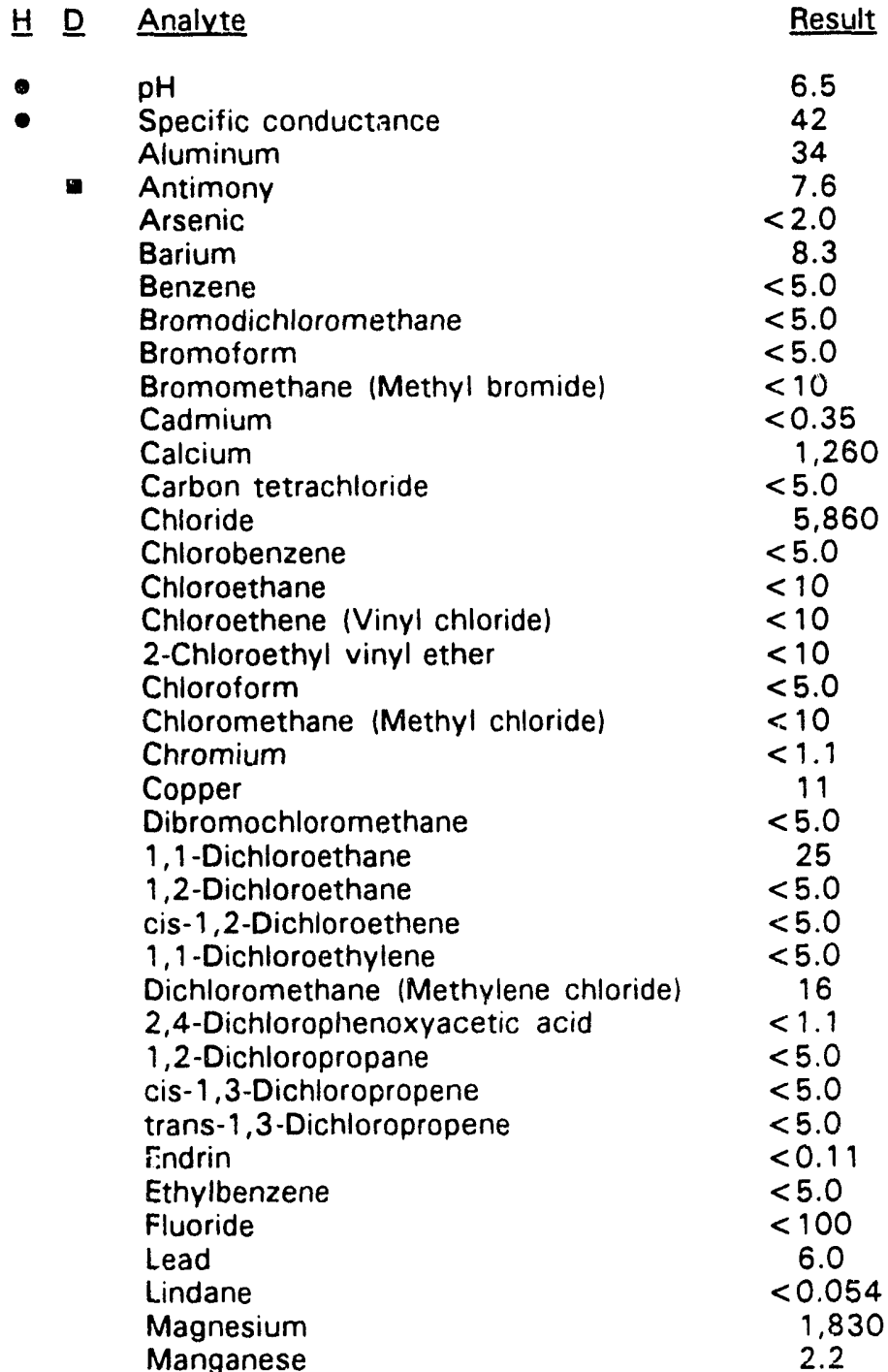

Time: $14: 35$

pH: 4.4

Alkalinity: $0 \mathrm{mg} / \mathrm{L}$

Water temperature: $19.7^{\circ} \mathrm{C}$ 
WELL LFW 16 collected on 05/28/92, laboratory analyses (cont.)

$\begin{array}{ll}\text { H. } & \text { Analyte } \\ \text { Mercury } \\ \text { Methoxychlor } \\ \text { Nickel } \\ \text { Nitrate as nitrogen } \\ \text { Potassium } \\ \text { Selenium } \\ \text { Silica } \\ \text { Silver } \\ \text { Sodium } \\ \text { Sulfate } \\ \text { 1,1,2,2-Tetrachloroethane } \\ \text { Tetrachloroethylene } \\ \text { Toluene } \\ \text { Total organic carbon } \\ \text { Total organic halogens } \\ \text { Toxaphene } \\ \text { 2,4,5-TP (Silvex) } \\ 1,1,1-\text { Trichloroethane } \\ \text { 1,1,2-Trichloroethane } \\ \text { Trichloroethylene } \\ \text { Trichlorofluoromethane } \\ \text { Vanadium } \\ \text { Gross alpha } \\ \text { Nonvolatile beta } \\ \text { Radium-226 } \\ \text { Tritium }\end{array}$

$\begin{aligned} & \text { Result } \\ &< 0.20 \\ &< 0.54 \\ & 6.1 \\ & 675 \\ & 424 \\ &<2.0 \\ & 8,820 \\ &<0.70 \\ & 1.910 \\ &<2,500 \\ &<5.0 \\ & 2.7 \\ &<5.0 \\ & 1,440 \\ & 89 \\ &<1.1 \\ &<0.57 \\ & 3.9 \\ & 1.1 \\ & 7.8 \\ &<5.0 \\ &<0.88 \\ &<3.0 \mathrm{E}+00 \\ &<5.0 \mathrm{E}+00 \\ & 5.3 \mathrm{E}-01 \pm 1.8 \mathrm{E}-01 \\ & 2.1 \mathrm{E}+01 \pm 8.6 \mathrm{E}-01\end{aligned}$

$\begin{array}{llll}\text { Unit } & \text { Flag } & \text { Lab } \\ \mu \mathrm{g} / \mathrm{L} & 0 & \text { WA } \\ \mu \mathrm{g} / \mathrm{L} & 0 & \text { WA } \\ \mu \mathrm{g} / \mathrm{L} & 0 & \text { WA } \\ \mu \mathrm{g} / \mathrm{L} & 0 & \text { WA } \\ \mu \mathrm{g} / \mathrm{L} & 0 & \text { WA } \\ \mu \mathrm{g} / \mathrm{L} & 0 & \text { WA } \\ \mu \mathrm{g} / \mathrm{L} & 0 & \text { WA } \\ \mu \mathrm{g} / \mathrm{L} & 0 & \text { WA } \\ \mu \mathrm{g} / \mathrm{L} & 0 & \text { WA } \\ \mu \mathrm{g} / \mathrm{L} & 0 & \text { WA } \\ \mu \mathrm{g} / \mathrm{L} & 0 & \text { WA } \\ \mu \mathrm{g} / \mathrm{L} & 1 & \text { WA } \\ \mu \mathrm{g} / \mathrm{L} & 0 & \text { WA } \\ \mu \mathrm{g} / \mathrm{L} & 0 & \text { WA } \\ \mu \mathrm{g} / \mathrm{L} & 2 & \text { WA } \\ \mu \mathrm{g} / \mathrm{L} & 0 & \text { WA } \\ \mu \mathrm{g} / \mathrm{L} & 0 & \text { WA } \\ \mu \mathrm{g} / \mathrm{L} & 0 & \text { WA } \\ \mu \mathrm{g} / \mathrm{L} & 0 & \text { WA } \\ \mu \mathrm{g} / \mathrm{L} & 2 & \text { WA } \\ \mu \mathrm{g} / \mathrm{L} & 0 & \text { WA } \\ \mu \mathrm{g} / \mathrm{L} & 0 & \text { WA } \\ \mathrm{pCi} / \mathrm{L} & 0 & \text { CN } \\ \mathrm{pCi} / \mathrm{L} & 0 & \text { CN } \\ \mathrm{pCi} / \mathrm{L} & 0 & \text { CN } \\ \mathrm{pCi} / \mathrm{mL} & 2 & \text { CN }\end{array}$

\section{WELL LFW 17}

$\begin{array}{llllll}\text { SRS Coord. } & \text { Lat/Longitude } & \text { Screen Zone Elevation } & \text { Top of Casing } & \text { Casing } & \text { Formation } \\ \text { N84602.8 } & 33.287295^{\circ} \mathrm{N} & 158.5-128.5 \mathrm{ft} \mathrm{msl} & 177.8 \mathrm{ft} \mathrm{msl} & 4 \text { " PVC } \\ \text { E45607.3 } & 81.711048^{\circ} \mathrm{W} & & & \end{array}$

\section{MEASUREMENTS CONDUCTED IN THE FIELD}

Sample date: 05/28/92

Depth to water: $23.09 \mathrm{ft}(7.04 \mathrm{~m})$ below TOC

Water elevation: $154.71 \mathrm{ft}(47.16 \mathrm{~m}) \mathrm{msl}$

Sp. conductance: $239 \mu \mathrm{S} / \mathrm{cm}$

Water evacuated before sampling: $69 \mathrm{gal}$

\section{LABORATORY ANALYSES}

$\begin{array}{ll}\text { H } & \text { Analyte } \\ & \text { pH } \\ \text { - } & \text { Specific conductance } \\ & \text { Aluminum } \\ \text { - Antimony } \\ \text { Arsenic } \\ \text { Barium } \\ \text { Benzene } \\ \text { Bromodichloromethane }\end{array}$

Time: 9:00

$\mathrm{pH}: 6.0$

Alkalinity: $70 \mathrm{mg} / \mathrm{L}$

Water temperature: $19.1^{\circ} \mathrm{C}$

$\begin{aligned} & \text { Result } \\ & 6.6 \\ & 187 \\ &< 15 \\ & 9.6 \\ & 14 \\ &< \\ & 4.0 \\ & 2.6 \\ &< 5.0\end{aligned}$

$\begin{array}{lll}\text { Unit } & \text { Flag } & \text { Lab } \\ \mathrm{pH} & 0 & \text { WA } \\ \mu \mathrm{S} / \mathrm{cm} & 0 & \text { WA } \\ \mu \mathrm{g} / \mathrm{L} & 0 & \text { WA } \\ \mu \mathrm{g} / \mathrm{L} & 2 & \text { WA } \\ \mu \mathrm{g} / \mathrm{L} & 0 & \text { WA } \\ \mu \mathrm{g} / \mathrm{L} & 0 & \text { WA } \\ \mu \mathrm{g} / \mathrm{L} & 1 & \text { WA } \\ \mu \mathrm{g} / \mathrm{L} & 0 & \text { WA }\end{array}$

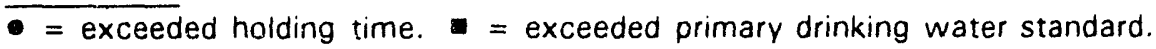


WELL LFW 17 collected on 05/28/92, laboratory analyses (cont.)

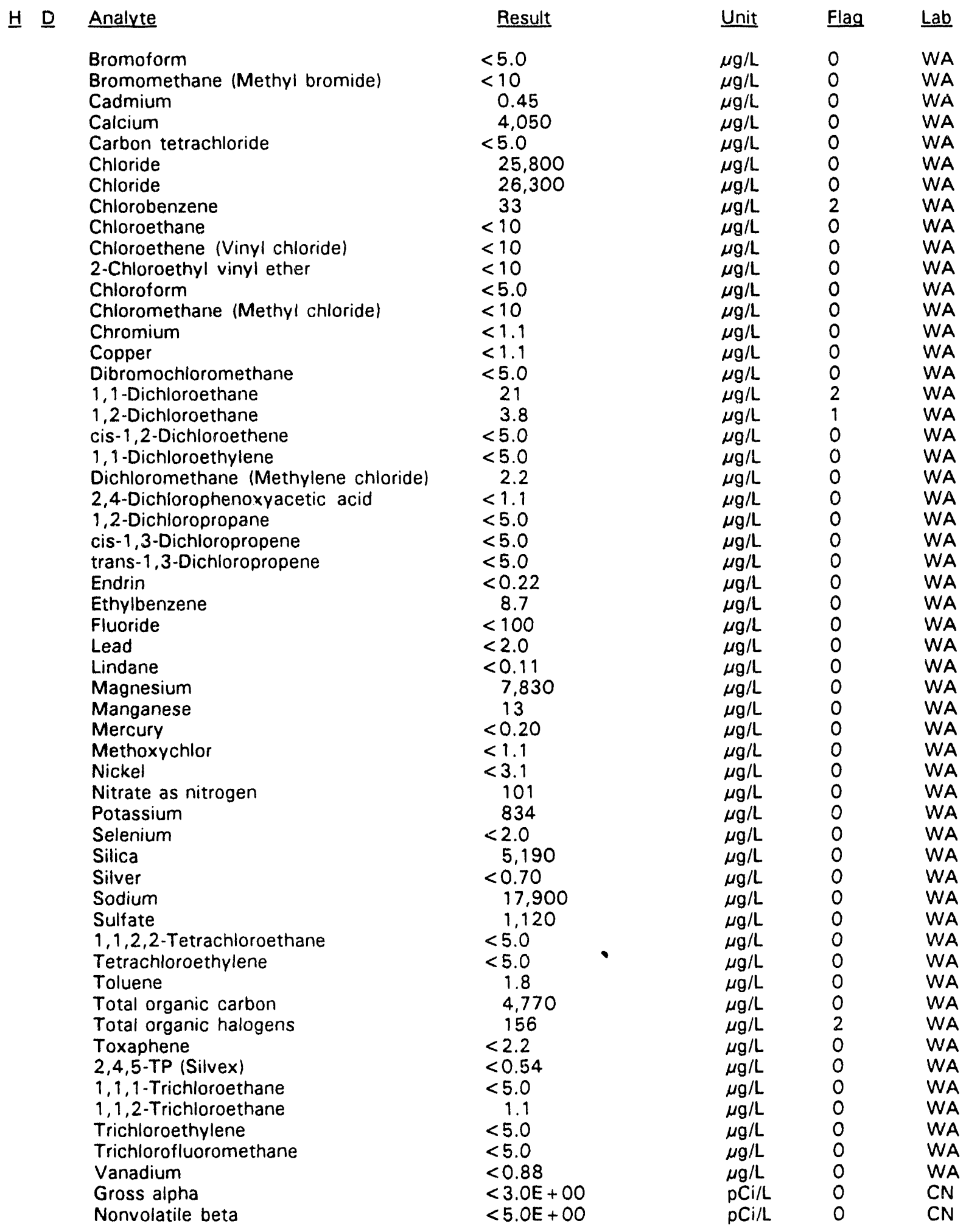

$\overline{- \text { = exceeded holding time. }} \mathbf{a}=$ exceeded primary drinking water standard. 
WELL LFW 17 collected on 05/28/92, laboratory analyses (cont.)

$\begin{array}{llllll}\underline{H} \underline{D} & \text { Analyte } & \text { Result } & \text { Unit } & \underline{\text { Flag }} & \underline{\text { Lab }} \\ & \text { Radium-226 } & 6.9 \mathrm{E}-01 \pm 2.1 \mathrm{E}-01 & \mathrm{pCi} / \mathrm{L} & 0 & \mathrm{CN} \\ \text { Tritium } & 8.4 \mathrm{E}+00 \pm 6.0 \mathrm{E}-01 & \mathrm{pCi} / \mathrm{mL} & 0 & \mathrm{CN}\end{array}$

\section{WELL LFW 18}

$\begin{array}{lllllll}\text { SRS Coord. Lat/Longitude } & \text { Screen Zone Elevation } & \text { Top of Casing } & & \text { Casing } & \text { Formation } \\ \text { N84577.3 } & 33.286997^{\circ} \mathrm{N} & 160.1-130.1 \mathrm{ft} \mathrm{msl} & 175.0 \mathrm{ft} \mathrm{msl} & \text { 4" PVC } \\ \text { E45459.4 } & 81.711388^{\circ} \mathrm{W} & & & \end{array}$

\section{MEASUREMENTS CONDUCTED IN THE FIELD}

Sample date: 05/28/92

Depth to water: $20.43 \mathrm{ft}(6.23 \mathrm{~m})$ below TOC

Water elevation: $154.57 \mathrm{ft}(47.11 \mathrm{~m}) \mathrm{msl}$

Sp. conductance: $259 \mu \mathrm{S} / \mathrm{cm}$

Water evacuated before sampling: $64 \mathrm{gal}$

\section{LABORATORY ANALYSES}

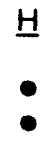

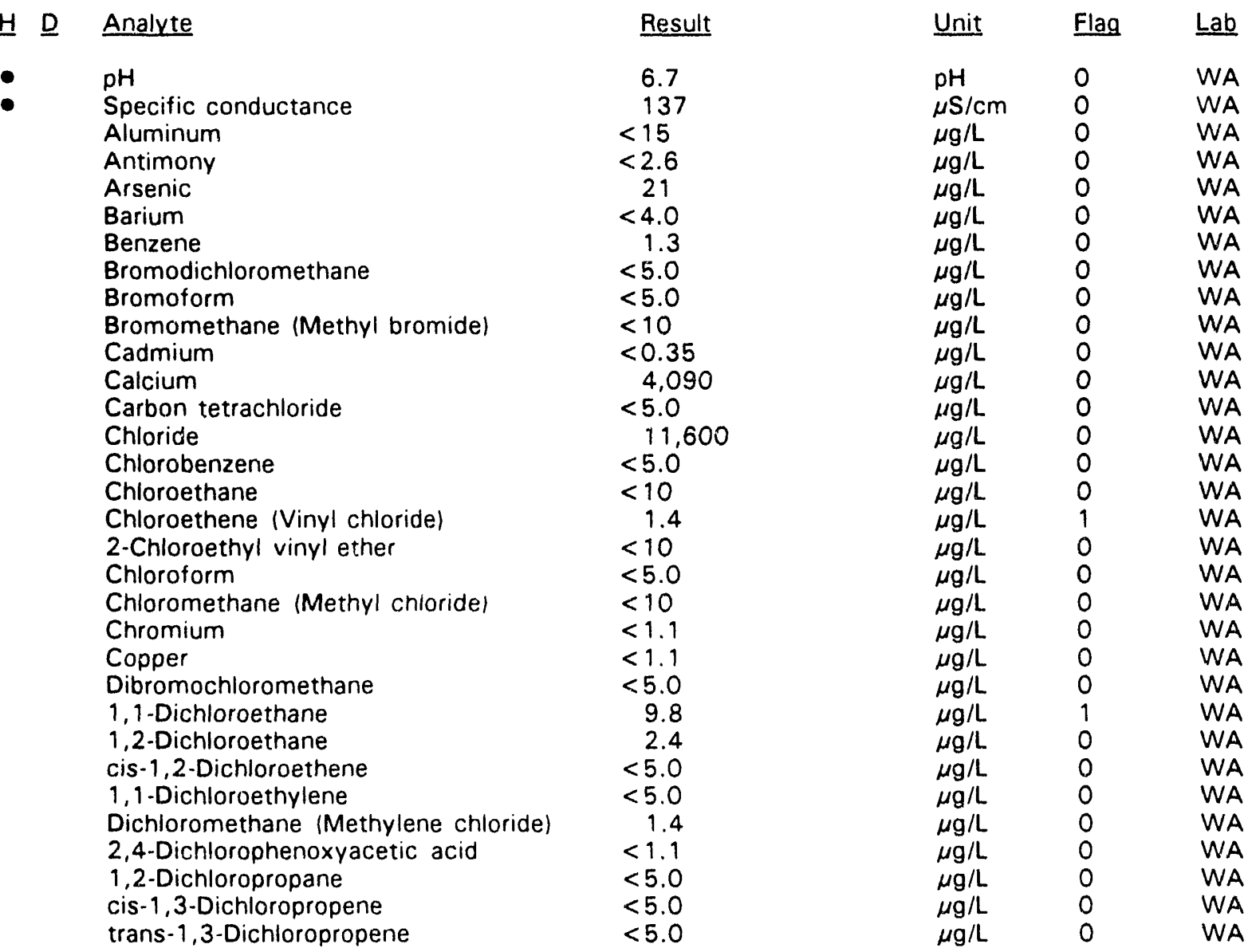

Time: $8: 15$

$\mathrm{pH}: 6.3$

Alkalinity: $95 \mathrm{mg} / \mathrm{L}$

Water temperature: $18.7^{\circ} \mathrm{C}$

$\overline{- \text { = exceeded holding time. }} \mathbf{-}=$ exceeded primary drinking water standard. 
WELL LFW 18 collected on $05 / 28 / 92$, laboratory analyses (cont.)

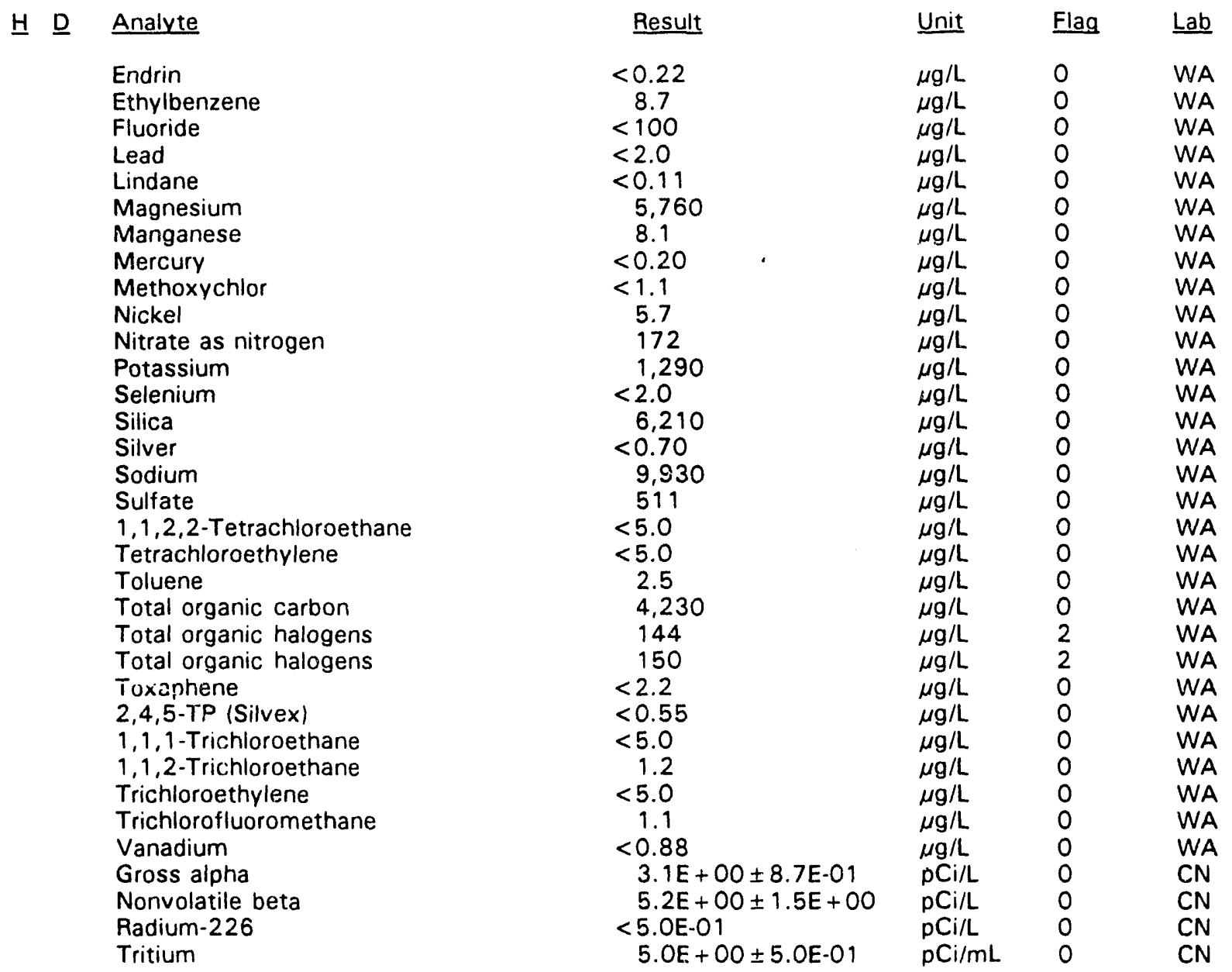

$\overline{- \text { = exceeded holding time. }} \cdot$ = exceeded primary drinking water standard. 
WELL LFW 19

$\begin{array}{llllll}\text { SRS Coord. } & \text { Lat/Longitude } & \text { Screen Zone Elevation } & \text { Top of Casing } & \text { Casing } & \text { Formation } \\ \text { N84817.2 } & 33.286998^{\circ} \mathrm{N} & 160.0-130.0 \mathrm{ft} \mathrm{msl} & 176.7 \mathrm{ft} \mathrm{msl} & 4 \text { " PVC } \\ \text { E45135.4 } & 81.712707^{\circ} \mathrm{W} & & & \end{array}$

\section{MEASUREMENTS CONDUCTED IN THE FIELD}

Sample date: 05/28/92

Depth to water: $20.73 \mathrm{ft}(6.32 \mathrm{~m})$ below TOC Water elevation: $155.97 \mathrm{ft}(47.54 \mathrm{~m}) \mathrm{msl}$

Sp. conductance: $19 \mu \mathrm{S} / \mathrm{cm}$

Water evacuated before sampling: $68 \mathrm{gal}$

\section{LABORATORY ANALYSES}

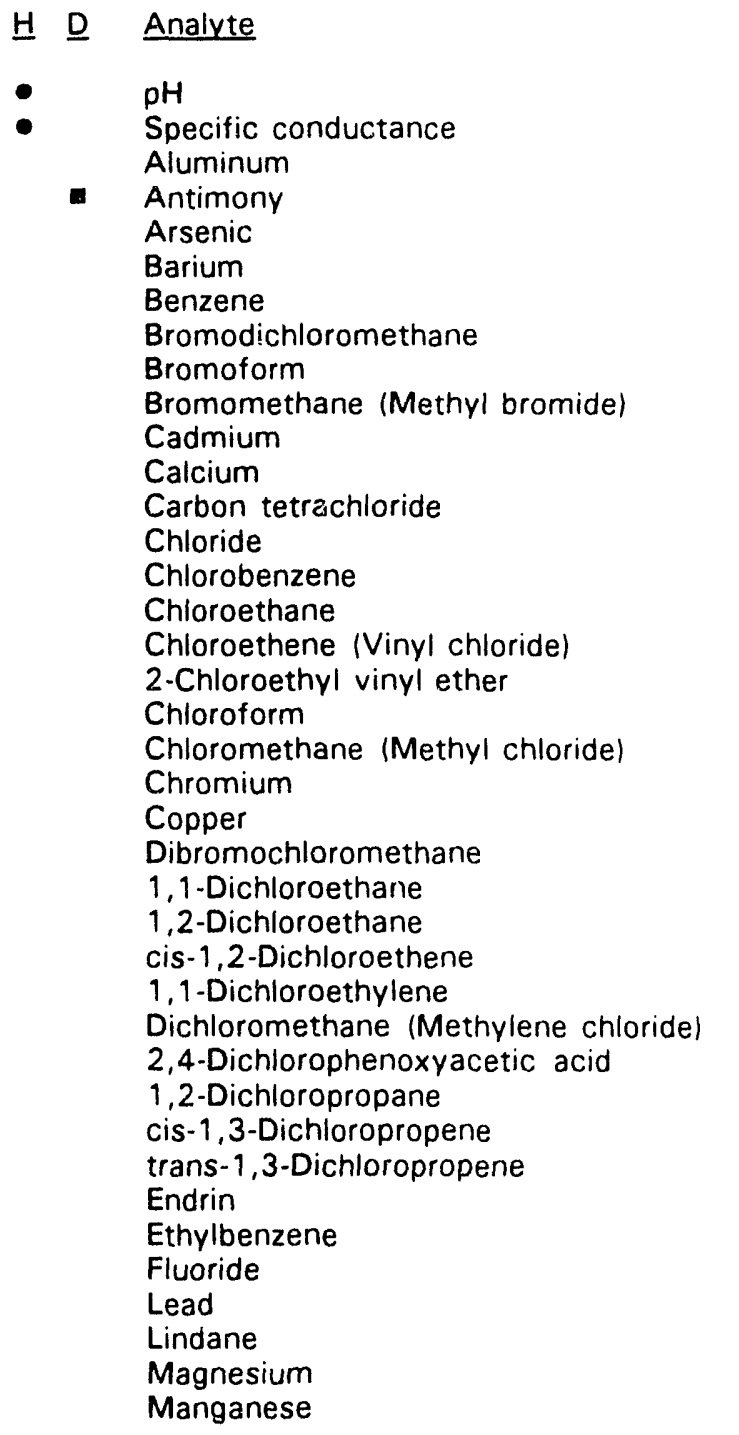

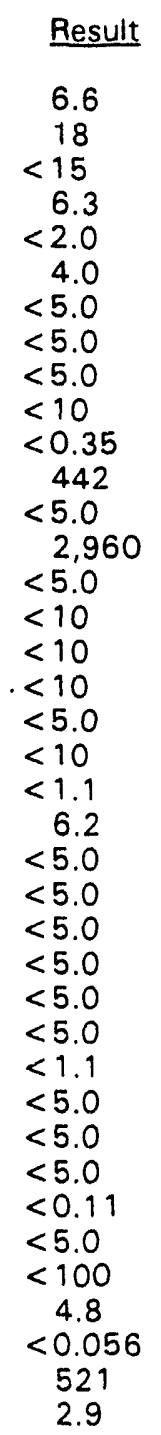

Time: $10: 00$

pH: 4.3

Alkalinity: $0 \mathrm{mg} / \mathrm{L}$

Water temperature: $18.5^{\circ} \mathrm{C}$ 
WELL LFW 19 collected on 05/28/92, laboratory analyses (cont.)

$\begin{array}{ll}\text { H. } & \text { Analyte } \\ \text { Mercury } \\ \text { Methoxychlor } \\ \text { Nickel } \\ \text { Nitrate as nitrogen } \\ \text { Potassiurn } \\ \text { Selenium } \\ \text { Silica } \\ \text { Silver } \\ \text { Sodium } \\ \text { Sulfate } \\ \text { 1,1,2,2-Tetrachloroethane } \\ \text { Tetrachloroethylene } \\ \text { Toluene } \\ \text { Total organic carbon } \\ \text { Total organic halogens } \\ \text { Toxaphene } \\ \text { 2,4,5-TP (Silvex) } \\ \text { 1,1,1-Trichloroethane } \\ \text { 1,1,2-Trichloroethane } \\ \text { Trichloroethylene } \\ \text { Trichlorofluoromethane } \\ \text { Vanadium } \\ \text { Gross alpha } \\ \text { Nonvolatile beta } \\ \text { Radium-226 } \\ \text { Tritium }\end{array}$

$\begin{array}{llll}\text { Result } & \underline{\text { Unit }} & \text { Flag } & \text { Lab } \\ <0.20 & \mu \mathrm{g} / \mathrm{L} & 0 & \text { WA } \\ <0.56 & \mu \mathrm{g} / \mathrm{L} & 0 & \text { WA } \\ <3.1 & \mu \mathrm{g} / \mathrm{L} & 0 & \text { WA } \\ 407 & \mu \mathrm{g} / \mathrm{L} & 0 & \text { WA } \\ 228 & \mu \mathrm{g} / \mathrm{L} & 0 & \text { WA } \\ <2.0 & \mu \mathrm{g} / \mathrm{L} & 0 & \text { WA } \\ 5,590 & \mu \mathrm{g} / \mathrm{L} & 0 & \text { WA } \\ <0.70 & \mu \mathrm{g} / \mathrm{L} & 0 & \text { WA } \\ 1,400 & \mu \mathrm{g} / \mathrm{L} & 0 & \text { WA } \\ 378 & \mu \mathrm{g} / \mathrm{L} & 0 & \text { WA } \\ <5.0 & \mu \mathrm{g} / \mathrm{L} & 0 & \text { WA } \\ <5.0 & \mu \mathrm{g} / \mathrm{L} & 0 & \text { WA } \\ <5.0 & \mu \mathrm{g} / \mathrm{L} & 0 & \text { WA } \\ 3,370 & \mu \mathrm{g} / \mathrm{L} & 0 & \text { WA } \\ 36 & \mu \mathrm{g} / \mathrm{L} & 1 & \text { WA } \\ <1.1 & \mu \mathrm{g} / \mathrm{L} & 0 & \text { WA } \\ <0.55 & \mu \mathrm{g} / \mathrm{L} & 0 & \text { WA } \\ <5.0 & \mu \mathrm{g} / \mathrm{L} & 0 & \text { WA } \\ 1.0 & \mu \mathrm{g} / \mathrm{L} & 0 & \text { WA } \\ <5.0 & \mu \mathrm{g} / \mathrm{L} & 0 & \text { WA } \\ <5.0 & \mu \mathrm{g} / \mathrm{L} & 0 & \text { WA } \\ <0.88 & \mu \mathrm{g} / \mathrm{L} & 0 & \text { WA } \\ 4.3 \mathrm{E}+00 \pm 7.7 \mathrm{E}-01 & \mathrm{pCi} / \mathrm{L} & 0 & \text { CN } \\ 6.9 \mathrm{E}+00 \pm 1.2 \mathrm{E}+00 & \mathrm{pCi} / \mathrm{L} & 0 & \text { CN } \\ 1.2 \mathrm{E}+00 \pm 2.6 \mathrm{E}-01 & \mathrm{pCi} / \mathrm{L} & 0 & \text { CN } \\ 2.4 \mathrm{E}+00 \pm 4.1 \mathrm{E}-01 & \mathrm{pCi} / \mathrm{mL} & 0 & \text { CN }\end{array}$

\section{WELL LFW 20}

\begin{tabular}{|c|c|c|c|c|}
\hline SRS Coord. & Lat/Longitude & Screen Zone Elevation & Top of Casing & Casing \\
\hline $\begin{array}{l}N 85262.6 \\
E 45582.9\end{array}$ & $\begin{array}{l}33.288714^{\circ} \mathrm{N} \\
81.712395^{\circ} \mathrm{W}\end{array}$ & $165.0-135.0 \mathrm{ft} \mathrm{msl}$ & $180.5 \mathrm{ft} \mathrm{msl}$ & VC \\
\hline
\end{tabular}

\section{MEASUREMENTS CONDUCTED IN THE FIELD}

Sample date: 05/29/92

Time: $14: 25$

Depth to water: $21.55 \mathrm{ft}(6.57 \mathrm{~m})$ below TOC

Water elevation: $158.95 \mathrm{ft}(48.45 \mathrm{~m}) \mathrm{msl}$

No water was evacuated before sampling.

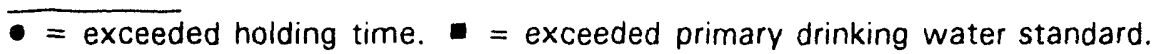


WELL LFW 21

\begin{tabular}{|c|c|c|c|c|c|}
\hline SRS Coord. & Lat/Longitude & Screen Zone Elevation & Top of Casing & Casing & Formation \\
\hline $\begin{array}{l}\text { N84178.3 } \\
\text { E46149.4 }\end{array}$ & $\begin{array}{l}33.287241^{\circ} \mathrm{N} \\
81.708796^{\circ} \mathrm{W}\end{array}$ & $158.9-128.9 \mathrm{ft} \mathrm{msl}$ & $175.6 \mathrm{ft} \mathrm{msl}$ & /C & \\
\hline
\end{tabular}

MEASUREMENTS CONDUCTED IN THE FIELD

Sample date: 05/29/92

Depth to water: $25.21 \mathrm{ft}(7.68 \mathrm{~m})$ below TOC Water elevation: $150.39 \mathrm{ft}(45.84 \mathrm{~m}) \mathrm{msl}$ Sp. conductance: $1413 \mu \mathrm{S} / \mathrm{cm}$

Water evacuated before sampling: $56 \mathrm{gal}$

\section{LABORATORY ANALYSES}

Time: 14:00

pH: 6.5

Alkalinity: $363 \mathrm{mg} / \mathrm{L}$

Water temperature: $18.8^{\circ} \mathrm{C}$

$$
\text { : }
$$

$\mathrm{pH}$

Specific conductance

Aluminum

Antimony

Arsenic

Barium

- Benzene

Bromodichloromethane

Bromoform

Bromomethane (Methyl bromide)

Cadmium

Calcium

Carbon tetrachloride

Chloride

Chlorobenzene

Chloroethane

Chloroethene (Vinyl chloride)

2-Chloroethyi vinyl ether

Chloroform

Chloromethane (Methyl chloride)

Chromium

Copper

Dibromochloromethane

1,1-Dichloroethane

1,2-Dichloroethane

cis-1,2-Dichloroethene

1,1-Dichloroethylene

Dichloromethane (Methylene chloride)

2,4-Dichlorophenoxyacetic acid

2,4-Dichlorophenoxyacetic acid

- 1,2-Dichloropropane

cis-1,3-Dichloropropene

trans-1,3-Dichloropropene

Endrin

Ethylbenzene

Fluoride

Fluoride

Lead

Lindane

\begin{tabular}{lll} 
Unit & Flag & Lab \\
\hline $\mathrm{pH}$ & 0 & WA \\
$\mu \mathrm{S} / \mathrm{cm}$ & 2 & WA \\
$\mu \mathrm{g} / \mathrm{L}$ & 0 & WA \\
$\mu \mathrm{g} / \mathrm{L}$ & 1 & WA \\
$\mu \mathrm{g} / \mathrm{L}$ & 0 & WA \\
$\mu \mathrm{g} / \mathrm{L}$ & 0 & WA \\
$\mu \mathrm{g} / \mathrm{L}$ & 2 & WA \\
$\mu \mathrm{g} / \mathrm{L}$ & 0 & WA \\
$\mu \mathrm{g} / \mathrm{L}$ & 0 & WA \\
$\mu \mathrm{g} / \mathrm{L}$ & 0 & WA \\
$\mu \mathrm{g} / \mathrm{L}$ & 0 & WA \\
$\mu \mathrm{g} / \mathrm{L}$ & 0 & WA \\
$\mu \mathrm{g} / \mathrm{L}$ & 0 & WA \\
$\mu \mathrm{g} / \mathrm{L}$ & 0 & WA \\
$\mu \mathrm{g} / \mathrm{L}$ & 0 & WA \\
$\mu \mathrm{g} / \mathrm{L}$ & 0 & WA \\
$\mu \mathrm{g} / \mathrm{L}$ & 0 & WA \\
$\mu \mathrm{g} / \mathrm{L}$ & 0 & WA \\
$\mu \mathrm{g} / \mathrm{L}$ & 0 & WA \\
$\mu \mathrm{g} / \mathrm{L}$ & 0 & WA \\
$\mu \mathrm{g} / \mathrm{L}$ & 0 & WA \\
$\mu \mathrm{g} / \mathrm{L}$ & 0 & WA \\
$\mu \mathrm{g} / \mathrm{L}$ & 0 & WA \\
$\mu \mathrm{g} / \mathrm{L}$ & 2 & WA \\
$\mu \mathrm{g} / \mathrm{L}$ & 1 & WA \\
$\mu \mathrm{g} / \mathrm{L}$ & 0 & WA \\
$\mu \mathrm{g} / \mathrm{L}$ & 1 & WA \\
$\mu \mathrm{g} / \mathrm{L}$ & 0 & WA \\
$\mu \mathrm{g} / \mathrm{L}$ & 0 & WA \\
$\mu \mathrm{g} / \mathrm{L}$ & 0 & WA \\
$\mu \mathrm{g} / \mathrm{L}$ & 2 & WA \\
$\mu \mathrm{g} / \mathrm{L}$ & 0 & WA \\
$\mu \mathrm{g} / \mathrm{L}$ & 0 & WA \\
$\mu \mathrm{g} / \mathrm{L}$ & 0 & WA \\
$\mu \mathrm{g} / \mathrm{L}$ & 0 & WA \\
$\mu \mathrm{g} / \mathrm{L}$ & 0 & WA \\
$\mu \mathrm{g} / \mathrm{L}$ & 0 & WA \\
$\mu \mathrm{g} / \mathrm{L}$ & 0 & WA \\
$\mu \mathrm{g} / \mathrm{L}$ & 0 & WA \\
& 0 &
\end{tabular}

- exceeded holding time. = exceeded primary drinking water standard. 
WELL LFW 21 collected on 05/29/92, laboratory analyses (cont.)

$\begin{array}{ll}\text { H. } & \text { Analyte } \\ \text { Magnesium } \\ \text { Manganese } \\ \text { Mercury } \\ \text { Methoxychlor } \\ \text { Nickel } \\ \text { Nitrate as nitrogen } \\ \text { Potassium } \\ \text { Selenium } \\ \text { Silica } \\ \text { Silver } \\ \text { Sodium } \\ \text { Sulfate } \\ \text { 1,1,2,2-Tetrachloroethane } \\ \text { Tetrachloroethylene } \\ \text { Toluene } \\ \text { Total organic carbon } \\ \text { Total organic carbon } \\ \text { Total organic halogens } \\ \text { Toxaphene } \\ 2,4,5-T P \text { (Silvex) } \\ \text { 2,4,5-TP (Silvex) } \\ \text { 1,1,1-Trichloroethane } \\ \text { 1,1,2-Trichloroethane } \\ \text { Trichloroethylene } \\ \text { Trichlorofluoromethane } \\ \text { Vanadium } \\ \text { Gross alpha } \\ \text { Nonvolatile beta } \\ \text { Radium-226 } \\ \text { Tritium }\end{array}$

$\begin{aligned} & \text { Result } \\ & 33,400 \\ 363 & <0 \\ < & 0.20 \\ < & 0.54 \\ < & 1.1 \\ < & \\ & 323 \\ < & 2.0 \\ & 7,800 \\ < & 0.70 \\ & 52,300 \\ & 421 \\ < & 5.0 \\ & 4.9 \\ & 250 \\ & 460,000 \\ & 439,000 \\ & 1.090 \\ < & 1.1 \\ < & 0.54 \\ < & 1.1 \\ & 3.8 \\ < & 5.0 \\ & 27 \\ 43 & <0.88 \\ & 1.0 \mathrm{E}+01 \pm 1.7 \mathrm{E}+00 \\ & 5.1 \mathrm{E}+00 \pm 1.0 \mathrm{E}-01 \\ & 2.3 \mathrm{E}+00 \pm 4.0 \mathrm{E}-01 \\ & 1.2 \mathrm{E}+01 \pm 6.9 \mathrm{E}-01 \\ & \end{aligned}$

$\begin{array}{llll}\text { Unit } & \text { Flag } & \text { Lab } \\ \mu \mathrm{g} / \mathrm{L} & 0 & \text { WA } \\ \mu \mathrm{g} / \mathrm{L} & 2 & \text { WA } \\ \mu \mathrm{g} / \mathrm{L} & 0 & \text { WA } \\ \mu \mathrm{g} / \mathrm{L} & 0 & \text { WA } \\ \mu \mathrm{g} / \mathrm{L} & 0 & \text { WA } \\ \mu \mathrm{g} / \mathrm{L} & 0 & \text { WA } \\ \mu \mathrm{g} / \mathrm{L} & 0 & \text { WA } \\ \mu \mathrm{g} / \mathrm{L} & 0 & \text { WA } \\ \mu \mathrm{g} / \mathrm{L} & 0 & \text { WA } \\ \mu \mathrm{g} / \mathrm{L} & 0 & \text { WA } \\ \mu \mathrm{g} / \mathrm{L} & 0 & \text { WA } \\ \mu \mathrm{g} / \mathrm{L} & 0 & \text { WA } \\ \mu \mathrm{g} / \mathrm{L} & 0 & \text { WA } \\ \mu \mathrm{g} / \mathrm{L} & 1 & \text { WA } \\ \mu \mathrm{g} / \mathrm{L} & 0 & \text { WA } \\ \mu \mathrm{g} / \mathrm{L} & 2 & \text { WA } \\ \mu \mathrm{g} / \mathrm{L} & 2 & \text { WA } \\ \mu \mathrm{g} / \mathrm{L} & 2 & \text { WA } \\ \mu \mathrm{g} / \mathrm{L} & 0 & \text { WA } \\ \mu \mathrm{g} / \mathrm{L} & 0 & \text { WA } \\ \mu \mathrm{g} / \mathrm{L} & 0 & \text { WA } \\ \mu \mathrm{g} / \mathrm{L} & 0 & \text { WA } \\ \mu \mathrm{g} / \mathrm{L} & 0 & \text { WA } \\ \mu \mathrm{g} / \mathrm{L} & 2 & \text { WA } \\ \mu \mathrm{g} / \mathrm{L} & 2 & \text { WA } \\ \mu \mathrm{g} / \mathrm{L} & 0 & \text { WA } \\ \mathrm{pC \textrm {C } / \mathrm { L }} & 1 & \text { CN } \\ \mathrm{pCi} / \mathrm{L} & 0 & \text { CN } \\ \mathrm{pCi} / \mathrm{L} & 0 & \text { CN } \\ \mathrm{pCi} / \mathrm{mL} & 1 & \text { CN }\end{array}$

\section{WELL LFW 22}

\begin{tabular}{|c|c|c|c|c|c|}
\hline SRS Coord. & Lat/Longitude & Screen Zone Elevation & Top of Casing & Casing & Formation \\
\hline $\begin{array}{l}\text { N84223.6 } \\
\text { E46325.2 }\end{array}$ & $\begin{array}{l}33.287628^{\circ} \mathrm{N} \\
81.708421^{\circ} \mathrm{W}\end{array}$ & $152.4-122.4$ & 174 & C & \\
\hline
\end{tabular}

MEASUREMENTS CONDUCTED IN THE FIELD

Sample date: 05/29/92

Depth to water: $23.47 \mathrm{ft}(7.15 \mathrm{~m})$ below TOC Water elevation: $150.73 \mathrm{ft}(45.94 \mathrm{~m}) \mathrm{msl}$

Sp. conductance: $768 \mu \mathrm{S} / \mathrm{cm}$

Water evacuated before sampling: $74 \mathrm{gal}$

\section{LABORATORY ANALYSES}

Time: $13: 30$

$\mathrm{pH}: 6.3$

Alkalinity: $234 \mathrm{mg} / \mathrm{L}$

Water temperature: $18.9^{\circ} \mathrm{C}$

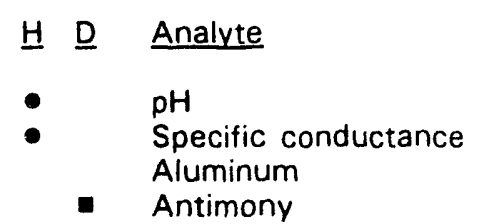

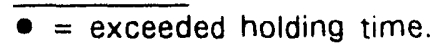

= exceeded primary drinking water standard.

Sanitary Landfill

$\begin{array}{llll}\text { Result } & \text { Unit } & \text { Flag } & \text { Lab } \\ 5.8 & & & \text { WH } \\ 459 & \mu S / \mathrm{cm} & 0 & \text { WA } \\ <15 & \mu \mathrm{g} / \mathrm{L} & 0 & \text { WA } \\ 10 & \mu \mathrm{g} / \mathrm{L} & 2 & \text { WA }\end{array}$


WELL LFW 22 collected on 05/29/92, laboratory analyses (cont.)

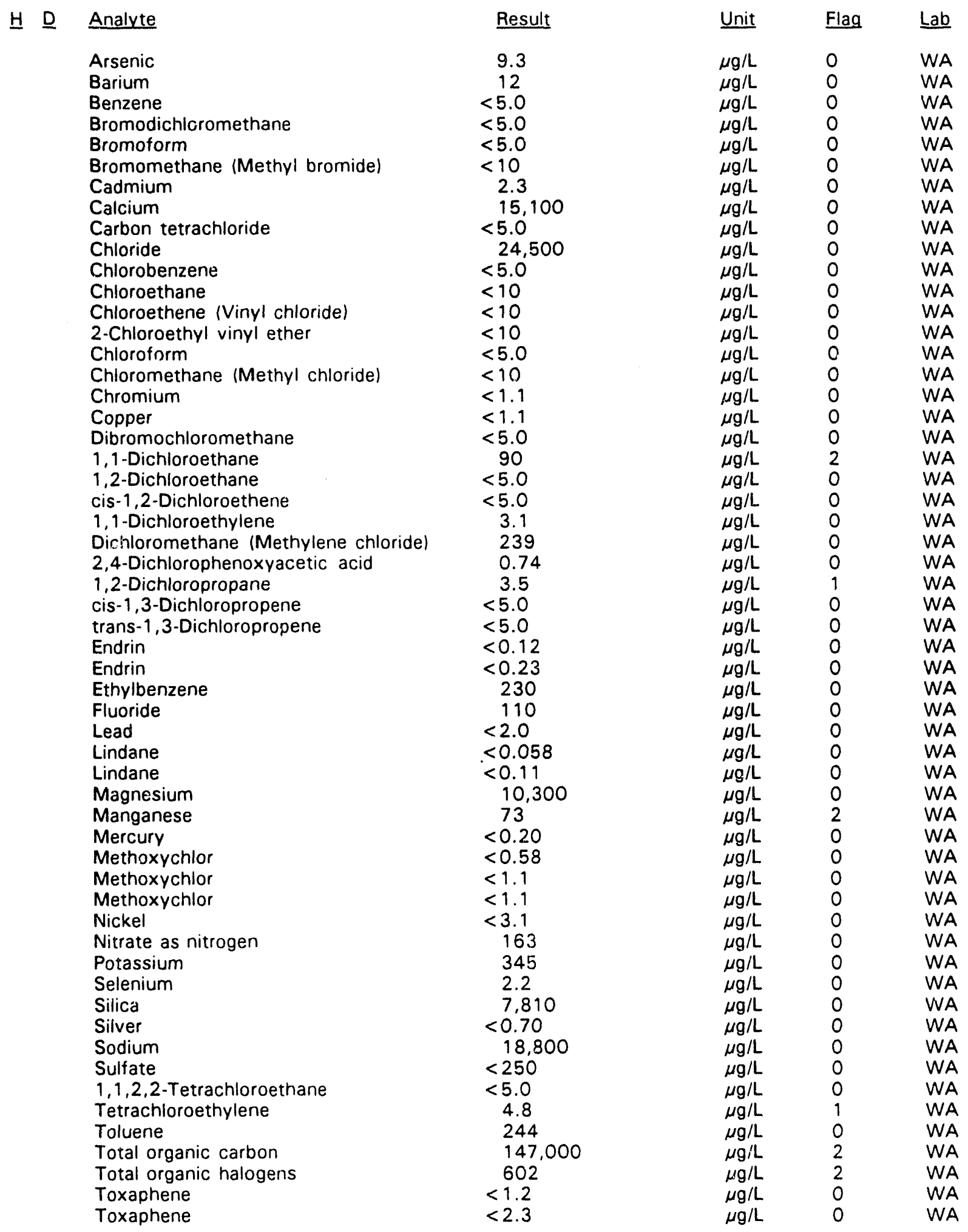

$\overline{-}=$ exceeded holding time. $=$ exceeded primary drinking water standard. 
WELL LFW 22 collected on 05/29/92, laboratory analyses (cont.)

H $\underline{\text { Analyte }}$

Toxaphene

2,4,5-TP (Silvex)

$1,1,1$-Trichloroethane

1,1,2-Trichloroethane

- Trichloroethylene

Trichlorofluoromethane

Vanadium

Gross alpha

Nonvolatile beta

Radium-226

Tritium

$\begin{aligned} & \text { Result } \\ < & 2.3 \\ < & 0.56 \\ & 5.1 \\ < & 5.0 \\ & 25 \\ & 44 \\ < & 0.88 \\ & 6.7 E+00 \pm 1.3 E+00 \\ < & 5.0 E+00 \\ & 9.5 E-01 \pm 2.4 E-01 \\ & 5.1 E+00 \pm 5.1 E-01\end{aligned}$

Unit

$\mu \mathrm{g} / \mathrm{L} \quad \mathrm{WA}$

$\mu \mathrm{g} / \mathrm{L} \quad \mathrm{O} \quad$ WA

$\mu \mathrm{g} / \mathrm{L} \quad 0 \quad$ WA

$\mu g / L \quad O \quad W A$

$\mu g / L \quad 2 \quad W A$

$\mu g / L \quad 2 \quad W A$

$\mu \mathrm{g} / \mathrm{L} \quad 0 \quad$ WA

$\mathrm{pCi} / \mathrm{L} \quad 0 \quad \mathrm{CN}$

$\mathrm{pCi} / \mathrm{L} \quad \mathrm{O} \quad \mathrm{CN}$

$\mathrm{pCi} / \mathrm{L} \quad \mathrm{O} \quad \mathrm{CN}$

$\mathrm{pCi} / \mathrm{rnL} \quad \mathrm{C} \quad \mathrm{CN}$

WELL LFW 23

$\begin{array}{lllllll}\text { SRS Coord. } & \text { Lat/Longitude } & \text { Screen Zone Elevation } & \text { Top of Casing } & \text { Casing } & \text { Formation } \\ \text { N84251.3 } & 33.287903^{\circ} \mathrm{N} & 155.1-125.1 \mathrm{ft} \mathrm{msl} & 171.8 \mathrm{ft} \mathrm{msl} & \text { 4" PVC } \\ \text { E46456.1 } & 81.708131^{\circ} \mathrm{W} & & & \end{array}$

MEASUREMENTS CONDUCTED IN THE CIELD

Sample date: 05/29/92

Depth to water: $20.85 \mathrm{ft}(6.36 \mathrm{~m})$ below TOC

Water elevation: $150.95 \mathrm{ft}(46.01 \mathrm{~m}) \mathrm{msl}$

Sp. conductance: $25 \mu \mathrm{S} / \mathrm{cm}$

Water evacuated before sampling: $68 \mathrm{gal}$

LABORATORY ANALYSES

\begin{tabular}{lc} 
- $D \quad$ Analyte & Result \\
\hline pH & 5.3 \\
Specific conductance & 22 \\
Aluminum & 21 \\
Antimony & $<2.6$ \\
Arsenic & $<2.0$ \\
Barium & $<4.0$ \\
Benzene & $<5.0$ \\
Benzene & $<5.0$ \\
Bromodichloromethane & $<5.0$ \\
Bromodichloromethane & $<5.0$ \\
Bromodichloromethane & $<5.0$ \\
Bromoform & $<5.0$ \\
Bromoform & $<5.0$ \\
Bromoform & $<5.0$ \\
Bromomethane (Methyl bromide) & $<10$ \\
Bromomethane (Methyl bromide) & $<10$ \\
Bromomethane (Methyl bromide) & $<10$ \\
Cadmium & 0.45 \\
Calcium & 416 \\
Carbon tetrachloride & $<5.0$ \\
Carbon tetrachloride & $<5.0$ \\
Carbon tetrachloride & $<5.0$ \\
Chloride & 2.070 \\
&
\end{tabular}

Time: $12: 50$

$\mathrm{pH}: 4.2$

Alkalinity: $0 \mathrm{mg} / \mathrm{L}$

Water temperature: $18.4^{\circ} \mathrm{C}$

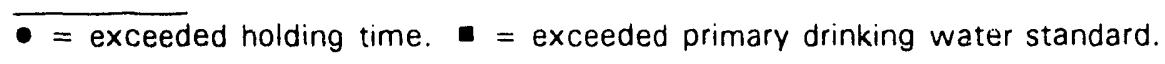

\begin{tabular}{lll} 
Unit & Flag & Lab \\
\hline $\mathrm{pH}$ & 0 & WA \\
$\mu \mathrm{S} / \mathrm{cm}$ & 0 & WA \\
$\mu \mathrm{g} / \mathrm{L}$ & 0 & WA \\
$\mu \mathrm{g} / \mathrm{L}$ & 0 & WA \\
$\mu \mathrm{g} / \mathrm{L}$ & 0 & WA \\
$\mu \mathrm{g} / \mathrm{L}$ & 0 & WA \\
$\mu \mathrm{g} / \mathrm{L}$ & 0 & WA \\
$\mu \mathrm{g} / \mathrm{L}$ & 0 & WA \\
$\mu \mathrm{g} / \mathrm{L}$ & 0 & WA \\
$\mu \mathrm{g} / \mathrm{L}$ & 0 & WA \\
$\mu \mathrm{g} / \mathrm{L}$ & 0 & WA \\
$\mu \mathrm{g} / \mathrm{L}$ & 0 & WA \\
$\mu \mathrm{g} / \mathrm{L}$ & 0 & WA \\
$\mu \mathrm{g} / \mathrm{L}$ & 0 & WA \\
$\mu \mathrm{g} / \mathrm{L}$ & 0 & WA \\
$\mu \mathrm{g} / \mathrm{L}$ & 0 & WA \\
$\mu \mathrm{g} / \mathrm{L}$ & 0 & WA \\
$\mu \mathrm{g} / \mathrm{L}$ & 0 & WA \\
$\mu \mathrm{g} / \mathrm{L}$ & 0 & WA \\
$\mu \mathrm{g} / \mathrm{L}$ & 0 & WA \\
$\mu \mathrm{g} / \mathrm{L}$ & 0 & WA \\
$\mu \mathrm{g} / \mathrm{L}$ & 0 & WA \\
$\mu \mathrm{g} / \mathrm{L}$ & 0 & WA
\end{tabular}


WFLL LFW 23 collected on 05/29/92, laboratory analyses (cont.)

H D Analyte

Chlorobenzene

Chlorobenzene

Chloroethane

Chloroethane

Chloroethane

Chloroethene (Vinyl chloricic)

Chloroethene (Vinyl chloride)

Chloroethene (Vinyl chloride)

2-Chloroethyl vinyl ether

2-Chloroethyl vinyl ether

2-Chloroethyl vinyl ether

Chloroform

Chloroform

Chloroform

Chioromethane (Methyl chloride)

Chloromethane (Methyl chloride)

Chloromethane (Methyl chloride)

Chromium

Copper

Dibromochloromethane

Dibromochloromethane

Dibromochloromethane

1.1-Diciloroethane

1,1-Dichloroethane

1.1-Dichloroethane

1,2-Dichloroethane

1,2-Dichloroethane

1,2-Dichloroethane

cis-1,2-Dichloroethene

cis-1,2-Dichloroethene

cis-1,2-Dichloroethene

1.1-Dichloroethylene

1,1-Dichloroethylene

Dichloromethane (Methylene chloride)

Dichloromethane (Methylene chloride)

Dichloromethane (Methylene chloride)

2,4-Dichlorophenoxyacetic acid

1,2-Dichloropropane

1,2-Dichloropropane

1,2-Dichloropropane

cis-1,3-Dichloropropene

cis-1,3-Dichloropropene

cis-1,3-Dichloropropene

trans-1,3-Dichloropropene

trans-1,3-Dichioropropene

trans-1,3-Dichloropropene

Endrin

Ethylbenzene

Ethylbenzene

Ethylbenzene

Fluoride

Lead

Lindane

Magnesium

Manganese

Mercury
Result

$<5.0$

$<5.0$

$<10$

$<10$

$<10$

$<10$

$<10$

$<10$

$<10$

$<10$

$<10$

$<5.0$

$<5.0$

$<5.0$

$<10$

$<10$

$<10$

$<1.1$

1.2

$<5.0$

$<5.0$

$<5.0$

1.8

27

3.5

$<5.0$

$<5.0$

$<5.0$

$<5.0$

$<5.0$

$<5.0$

$<5.0$

$<5.0$

6.1

7.9

4.4

$<1.1$

$<5.0$

$<5.0$

$<5.0$

$<5.0$

$<5.0$

$<5.0$

$<5.0$

$<5.0$

$<5.0$

$<0.11$

$<5.0$

$<5.0$

$<5.0$

$<100$

7.1

$<0.056$

635

2.2

$<0.20$

\begin{tabular}{|c|c|c|}
\hline Unit & Flag & $\underline{L a b}$ \\
\hline$\mu g / L$ & 0 & WA \\
\hline$\mu g / L$ & 0 & WA \\
\hline$\mu g / L$ & 0 & WA \\
\hline$\mu \mathrm{g} / \mathrm{L}$ & 0 & WA \\
\hline$\mu g / L$ & 0 & WA \\
\hline$\mu g / L$ & 0 & WA \\
\hline$\mu g / L$ & 0 & WA \\
\hline$\mu g / L$ & 0 & WA \\
\hline$\mu g / L$ & 0 & WA \\
\hline$\mu g / L$ & 0 & WA \\
\hline$\mu g / L$ & 0 & WA \\
\hline$\mu g / L$ & 0 & WA \\
\hline$\mu_{g} / L$ & 0 & WA \\
\hline$\mu g / L$ & 0 & WA \\
\hline$\mu g / L$ & 0 & WA \\
\hline$\mu g / L$ & 0 & WA \\
\hline$\mu g / L$ & 0 & WA \\
\hline$\mu g / L$ & 0 & WA \\
\hline$\mu \mathrm{g} / \mathrm{L}$ & 0 & WA \\
\hline$\mu g / L$ & C & WA \\
\hline$\mu g / L$ & 0 & WA \\
\hline$\mu g / L$ & 0 & WA \\
\hline$\mu \mathrm{g} / \mathrm{L}$ & 0 & WA \\
\hline$\mu \mathrm{g} / \mathrm{L}$ & 0 & WA \\
\hline$\mu g / L$ & 0 & WA \\
\hline$\mu g / L$ & 0 & WA \\
\hline$\mu g / L$ & 0 & WA \\
\hline$\mu g / L$ & 0 & WA \\
\hline$\mu g / L$ & 0 & WA \\
\hline$\mu g / L$ & 0 & WA \\
\hline$\mu g / L$ & 0 & WA \\
\hline$\mu g / L$ & 0 & WA \\
\hline$\mu g / L$ & 0 & WA \\
\hline$\mu g / L$ & 0 & WA \\
\hline$\mu g / L$ & 0 & WA \\
\hline$\mu g / L$ & 0 & WA \\
\hline$\mu g / L$ & 0 & WA \\
\hline$\mu g / L$ & 0 & WA \\
\hline$\mu g / L$ & 0 & WA \\
\hline$\mu g / L$ & 0 & WA \\
\hline$\mu g / L$ & 0 & WA \\
\hline$\mu g / L$ & 0 & WA \\
\hline$\mu g / L$ & 0 & WA \\
\hline$\mu g / L$ & 0 & WA \\
\hline$\mu g / L$ & 0 & WA \\
\hline$\mu g / L$ & 0 & WA \\
\hline$\mu g / L$ & 0 & WA \\
\hline$\mu g / L$ & 0 & WA \\
\hline$\mu g / L$ & 0 & WA \\
\hline$\mu g / L$ & 0 & WA \\
\hline$\mu g / L$ & 0 & WA \\
\hline$\mu g / L$ & 0 & WA \\
\hline$\mu g / L$ & 0 & WA \\
\hline$\mu g / L$ & 0 & WA \\
\hline$\mu g / L$ & 0 & WA \\
\hline & 0 & WA \\
\hline
\end{tabular}

- = exceeded holding time. = exceeded primary drinking water standard. 
WELL LFW 23 collected on 05/29/92, laboratory analyses (cont.)

\section{H D Analyte}

Methoxychlor

Nickel

Nitrate as nitrogen

Potassium

Selenium

Silica

Silver

Sodium

Sulfate

1,1,2,2-Tetrachloroethane

$1,1,2,2$-Tetrachloroethane

$1,1,2,2$-Tetrachloroethane

Tetrachloroethylene

Tetrachloroethylene

Tetrachloroethylene

Toluene

Toluene

Total organic carbon

Total organic halogens

Toxaphene

2,4,5-TP (Silvex)

1,1,1-Trichloroethane

1,1,1-Trichloroethane

1,1,1-Trichloroethane

1,1,2-Trichloroethane

1,1,2-Trichloroethane

1,1,2-Trichloroethane

Trichloroethylene

Trichloroethylene

Trichlorofluoromethane

Trichlorofluoromethane

Trichlorofluoromethane

Vanadium

Gross alpha

Nonvolatile beta

Radium-226

Tritium
Result

$<0.56$

4.1

816

368

$<2.0$

7,160

$<0.70$

2,010

$<250$

$<5.0$

$<5.0$

$<5.0$

$<5.0$

$<5.0$

$<5.0$

$<5.0$

$<5.0$

910

33

$<1.1$

$<0.55$

1.1

1.0

$<5.0$

$<5.0$

$<5.0$

$<5.0$

$<5.0$

$<5.0$

2.1

3.3

3.1

$<0.88$

$<3.0 E+00$

$<5.0 E+00$

1. $2 \mathrm{E}+00 \pm 2.8 \mathrm{E}-01$

$<2.0 E+O 0$

\begin{tabular}{|c|c|c|}
\hline Unit & Flag & Lab \\
\hline$\mu g / L$ & 0 & WA \\
\hline$\mu \mathrm{g} / \mathrm{L}$ & 0 & WA \\
\hline$\mu g / L$ & 0 & WA \\
\hline$\mu g / L$ & 0 & WA \\
\hline$\mu g / L$ & 0 & WA \\
\hline$\mu \mathrm{g} / \mathrm{L}$ & 0 & WA \\
\hline$\mu g / L$ & 0 & WA \\
\hline$\mu g / L$ & 0 & WA \\
\hline$\mu g / L$ & 0 & WA \\
\hline$\mu \mathrm{g} / \mathrm{L}$ & 0 & WA \\
\hline$\mu g / L$ & 0 & WA \\
\hline$\mu g / L$ & 0 & WA \\
\hline$\mu g / L$ & 0 & WA \\
\hline$\mu g / L$ & 0 & WA \\
\hline$\mu \mathrm{g} / \mathrm{L}$ & 0 & WA \\
\hline$\mu g / L$ & 0 & WA \\
\hline$\mu \mathrm{g} / \mathrm{L}$ & 0 & WA \\
\hline$\mu g / L$ & 0 & WA \\
\hline$\mu \mathrm{g} / \mathrm{L}$ & 1 & WA \\
\hline$\mu g / L$ & 0 & WA \\
\hline$\mu g / L$ & 0 & WA \\
\hline$\mu g / L$ & 0 & WA \\
\hline$\mu \mathrm{g} / \mathrm{L}$ & 0 & WA \\
\hline$\mu \mathrm{g} / \mathrm{L}$ & 0 & WA \\
\hline$\mu g / L$ & 0 & WA \\
\hline$\mu g / L$ & 0 & WA \\
\hline$\mu g / L$ & 0 & WA \\
\hline$\mu g / L$ & 0 & WA \\
\hline$\mu g / L$ & 0 & WA \\
\hline$\mu g / L$ & 0 & WA \\
\hline$\mu g / L$ & 0 & WA \\
\hline$\mu g / L$ & 0 & WA \\
\hline$\mu g / L$ & 0 & WA \\
\hline pCi/L & 0 & $\mathrm{CN}$ \\
\hline $\mathrm{pCi} / \mathrm{L}$ & 0 & $C N$ \\
\hline i/L & 0 & CN \\
\hline $\mathrm{pCi} / \mathrm{mL}$ & 0 & $\mathrm{CN}$ \\
\hline
\end{tabular}

$\overline{0}=$ exceeded holding time.

- = exceeded primary drinking water standard. 


\section{WELL LFW 24}

\begin{tabular}{|c|c|c|c|c|}
\hline SRS Coord. & Lat/Longitude & Screen Zone Elevation & Top of Casing & Casing \\
\hline $\begin{array}{l}\text { N84544.2 } \\
\text { E46520.8 }\end{array}$ & $\begin{array}{l}33.288656^{\circ} \mathrm{N} \\
81.708530^{\circ} \mathrm{W}\end{array}$ & $154.5-124.5$ & $171.3 \mathrm{ft} \mathrm{msl}$ & 4" PVC \\
\hline
\end{tabular}

\section{MEASUREMENTS CONDUCTED IN THE FIELD}

Sample date: 05/29/92

Depth to water: $17.93 \mathrm{ft}(5.47 \mathrm{~m})$ below TOC Water elevation: $153.37 \mathrm{ft}(46.75 \mathrm{~m}) \mathrm{msl}$

Sp. conductance: $17 \mu 5 / \mathrm{cm}$

Water evacuated before sampling: $76 \mathrm{gal}$

\section{LABORATORY ANALYSES}

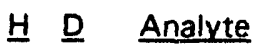

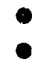

$\mathrm{pH}$
Specific conductance

Aluminum

Antimony

Arsenic

Barium

Benzene

Bromodichloromethane

Bromoform

Bromomethane (Methyl bromide)

Cadmium

Calcium

Carbon tetrachloride

Chloride

Chlorobenzene

Chloroethane

Chloroethene (Vinyl chloride)

2-Chloroethyl vinyl ether

Chloroform

Chloromethane (Methyl chloride)

Chromium

Copper

Dibromochloromethane

1,1-Dichloroethane

1,2-Dichloroethane

cis-1,2-Dichloroethene

1,1-Dichloroethylene

Dichloromethane (Methylene chloride)

2,4-Dichlorophenoxyacetic acid

1,2-Dichloropropane

cis-1,3-Dichloropropene

trans-1,3-Dichloropropene

Endrin

Ethylbenzene

Fluoride

Lead

Lindane

Magnesium

Manganese
Time: 12:15

$\mathrm{pH}: 4.2$

Alkalinity: $0 \mathrm{mg} / \mathrm{L}$

Water temperature: $18.1^{\circ} \mathrm{C}$

\begin{tabular}{llll} 
Result & Unit & Flag & Lab \\
\hline & & & \\
5.3 & $\mathrm{pH}$ & 0 & WA \\
15 & $\mu \mathrm{S} / \mathrm{cm}$ & 0 & WA \\
$<15$ & $\mu \mathrm{g} / \mathrm{L}$ & 0 & WA \\
$<2.6$ & $\mu \mathrm{g} / \mathrm{L}$ & 0 & WA \\
$<2.0$ & $\mu \mathrm{g} / \mathrm{L}$ & 0 & WA \\
$<4.0$ & $\mu \mathrm{g} / \mathrm{L}$ & 0 & WA \\
$<5.0$ & $\mu \mathrm{g} / \mathrm{L}$ & 0 & WA \\
$<5.0$ & $\mu \mathrm{g} / \mathrm{L}$ & 0 & WA \\
$<5.0$ & $\mu \mathrm{g} / \mathrm{L}$ & 0 & WA \\
$<10$ & $\mu \mathrm{g} / \mathrm{L}$ & 0 & WA \\
0.45 & $\mu \mathrm{g} / \mathrm{L}$ & 0 & WA \\
293 & $\mu \mathrm{g} / \mathrm{L}$ & 0 & WA \\
$<5.0$ & $\mu \mathrm{g} / \mathrm{L}$ & 0 & WA \\
1.860 & $\mu \mathrm{g} / \mathrm{L}$ & 0 & WA \\
$<5.0$ & $\mu \mathrm{g} / \mathrm{L}$ & 0 & WA \\
$<10$ & $\mu \mathrm{g} / \mathrm{L}$ & 0 & WA \\
$<10$ & $\mu \mathrm{g} / \mathrm{L}$ & 0 & WA \\
$<10$ & $\mu \mathrm{g} / \mathrm{L}$ & 0 & WA \\
$<5.0$ & $\mu \mathrm{g} / \mathrm{L}$ & 0 & WA \\
$<10$ & $\mu \mathrm{g} / \mathrm{L}$ & 0 & WA \\
1.3 & $\mu \mathrm{g} / \mathrm{L}$ & 0 & WA \\
11 & $\mu \mathrm{g} / \mathrm{L}$ & 0 & WA \\
$<5.0$ & $\mu \mathrm{g} / \mathrm{L}$ & 0 & WA \\
$<5.0$ & $\mu \mathrm{g} / \mathrm{L}$ & 0 & WA \\
$<5.0$ & $\mu \mathrm{g} / \mathrm{L}$ & 0 & WA \\
$<5.0$ & $\mu \mathrm{g} / \mathrm{L}$ & 0 & WA \\
$<5.0$ & $\mu \mathrm{g} / \mathrm{L}$ & 0 & WA \\
5.7 & $\mu \mathrm{g} / \mathrm{L}$ & 0 & WA \\
$<1.1$ & $\mu \mathrm{g} / \mathrm{L}$ & 0 & WA \\
$<5.0$ & $\mu \mathrm{g} / \mathrm{L}$ & 0 & WA \\
$<5.0$ & $\mu \mathrm{g} / \mathrm{L}$ & 0 & WA \\
$<5.0$ & $\mu \mathrm{g} / \mathrm{L}$ & 0 & WA \\
$<0.11$ & $\mu \mathrm{g} / \mathrm{L}$ & 0 & WA \\
$<5.0$ & $\mu \mathrm{g} / \mathrm{L}$ & 0 & WA \\
$<100$ & $\mu \mathrm{g} / \mathrm{L}$ & 0 & WA \\
4.3 & $\mu \mathrm{g} / \mathrm{L}$ & 0 & WA \\
$<0.057$ & $\mu \mathrm{g} / \mathrm{L}$ & 0 & WA \\
506 & $\mu \mathrm{g} / \mathrm{L}$ & 0 & WA \\
1.8 & & 0 & WA \\
& & &
\end{tabular}

$\overline{-=\text { exceeded holding time. }}$ = exceeded primary drinking water standard. 
WELL. LFW 24 collected on 05/29/92, laboratory analyses (cont.)

$\begin{array}{ll}\text { H. } & \text { Analyte } \\ \text { Mercury } \\ \text { Methoxychlor } \\ \text { Nickel } \\ \text { Nitrate as nitrogen } \\ \text { Nitrate as nitrogen } \\ \text { Potassium } \\ \text { Selenium } \\ \text { Silica } \\ \text { Silver } \\ \text { Sodium } \\ \text { Sulfate } \\ 1,1,2,2-\text { Tetrachloroethane } \\ \text { Tetrachloroethylene } \\ \text { Toluene } \\ \text { Total organic carbon } \\ \text { Total organic halogens } \\ \text { Toxaphene } \\ 2,4,5-T P \text { (Silvex) } \\ 1,1,1-\text { Trichloroethane } \\ \text { 1,1,2-Trichloroethane } \\ \text { Trichloroethylene } \\ \text { Trichlorofluoromethane } \\ \text { Vanadium } \\ \text { Gross alpha } \\ \text { Nonvolatile beta } \\ \text { Radium-226 } \\ \text { Radium-226 } \\ \text { Tritium }\end{array}$

$\begin{array}{llll}\text { Result } & \underline{\text { Unit }} & \underline{\text { Flag }} & \underline{\text { Lab }} \\ <0.20 & \mu \mathrm{g} / \mathrm{L} & 0 & \text { WA } \\ <0.57 & \mu \mathrm{g} / \mathrm{L} & 0 & \text { WA } \\ 4.8 & \mu \mathrm{g} / \mathrm{L} & 0 & \text { WA } \\ 495 & \mu \mathrm{g} / \mathrm{L} & 0 & \text { WA } \\ 495 & \mu \mathrm{g} / \mathrm{L} & 0 & \text { WA } \\ 411 & \mu \mathrm{g} / \mathrm{L} & 0 & \text { WA } \\ <2.0 & \mu \mathrm{g} / \mathrm{L} & 0 & \text { WA } \\ 5.470 & \mu \mathrm{g} / \mathrm{L} & 0 & \text { WA } \\ <0.70 & \mu \mathrm{g} / \mathrm{L} & 0 & \text { WA } \\ 1.140 & \mu \mathrm{g} / \mathrm{L} & 0 & \text { WA } \\ <250 & \mu \mathrm{g} / \mathrm{L} & 0 & \text { WA } \\ <5.0 & \mu \mathrm{g} / \mathrm{L} & 0 & \text { WA } \\ <5.0 & \mu \mathrm{g} / \mathrm{L} & 0 & \text { WA } \\ <5.0 & \mu \mathrm{g} / \mathrm{L} & 0 & \text { WA } \\ <500 & \mu \mathrm{g} / \mathrm{L} & 0 & \text { WA } \\ <20 & \mu \mathrm{g} / \mathrm{L} & 0 & \text { WA } \\ <1.1 & \mu \mathrm{g} / \mathrm{L} & 0 & \text { WA } \\ <0.56 & \mu \mathrm{g} / \mathrm{L} & 0 & \text { WA } \\ <5.0 & \mu \mathrm{g} / \mathrm{L} & 0 & \text { WA } \\ <5.0 & \mu \mathrm{g} / \mathrm{L} & 0 & \text { WA } \\ <5.0 & \mu \mathrm{g} / \mathrm{L} & 0 & \text { WA } \\ <5.0 & \mu \mathrm{g} / \mathrm{L} & 0 & \text { WA } \\ <0.88 & \mu \mathrm{g} / \mathrm{L} & 0 & \text { WA } \\ <3.0 \mathrm{E}+00 & \mathrm{pCi} / \mathrm{L} & 0 & \text { CN } \\ 5.4 \mathrm{E}+00 \pm 7.3 \mathrm{E}-01 & \mathrm{pCi} / \mathrm{L} & 0 & \text { CN } \\ 9.9 \mathrm{E}-01 \pm 3.2 \mathrm{E}-01 & \mathrm{pCi} / \mathrm{L} & 0 & \text { CN } \\ 7.0 \mathrm{E}-01 \pm 2.1 \mathrm{E}-01 & \mathrm{pCi} / \mathrm{L} & 0 & \text { CN } \\ 2.4 \mathrm{E}+00 \pm 4.2 \mathrm{E}-01 & \mathrm{pCi} / \mathrm{mL} & 0 & \text { CN }\end{array}$

\section{WELL LFW 25}

$\begin{array}{llllll}\text { SRS Coord. } & \text { Lat/Longitude } & \text { Screen Zone Elevation } & \text { Top of Casing } & \text { Casing } & \text { Formation } \\ \text { N84967.2 } & 33.2894366^{\circ} \mathrm{N} & 153.2-123.2 \mathrm{ft} \mathrm{msl} & 174.7 \mathrm{ft} \mathrm{msl} & \text { 4" PVC } \\ \text { E46425.7 } & 81.709602^{\circ} \mathrm{W} & & & \end{array}$

\section{MEASUREMENTS CONDUCTED IN THE FIELD}

Sample date: 06/02/92

Depth to water: $18.77 \mathrm{ft}(5.72 \mathrm{~m})$ below TOC

Water elevation: $155.93 \mathrm{ft}(47.53 \mathrm{~m}) \mathrm{msl}$

Sp. conductance: $16 \mu \mathrm{S} / \mathrm{cm}$

Water evacuated before sampling: $86 \mathrm{gal}$

\section{LABORATORY ANALYSES}

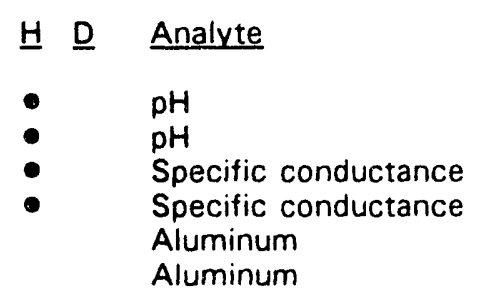

\begin{tabular}{l} 
Result \\
\hline 4.7 \\
4.7 \\
15 \\
15 \\
19 \\
18
\end{tabular}

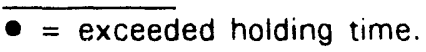

- exceeded primary drinking water standard.

Sanitary Landfill

Time: 9:00

$\mathrm{pH}: 4.0$

Alkalinity: $0 \mathrm{mg} / \mathrm{L}$

Water temperature: $18.0^{\circ} \mathrm{C}$ 
WELL LFW 25 collected on 06/02/92, laboratory analyses (cont.)

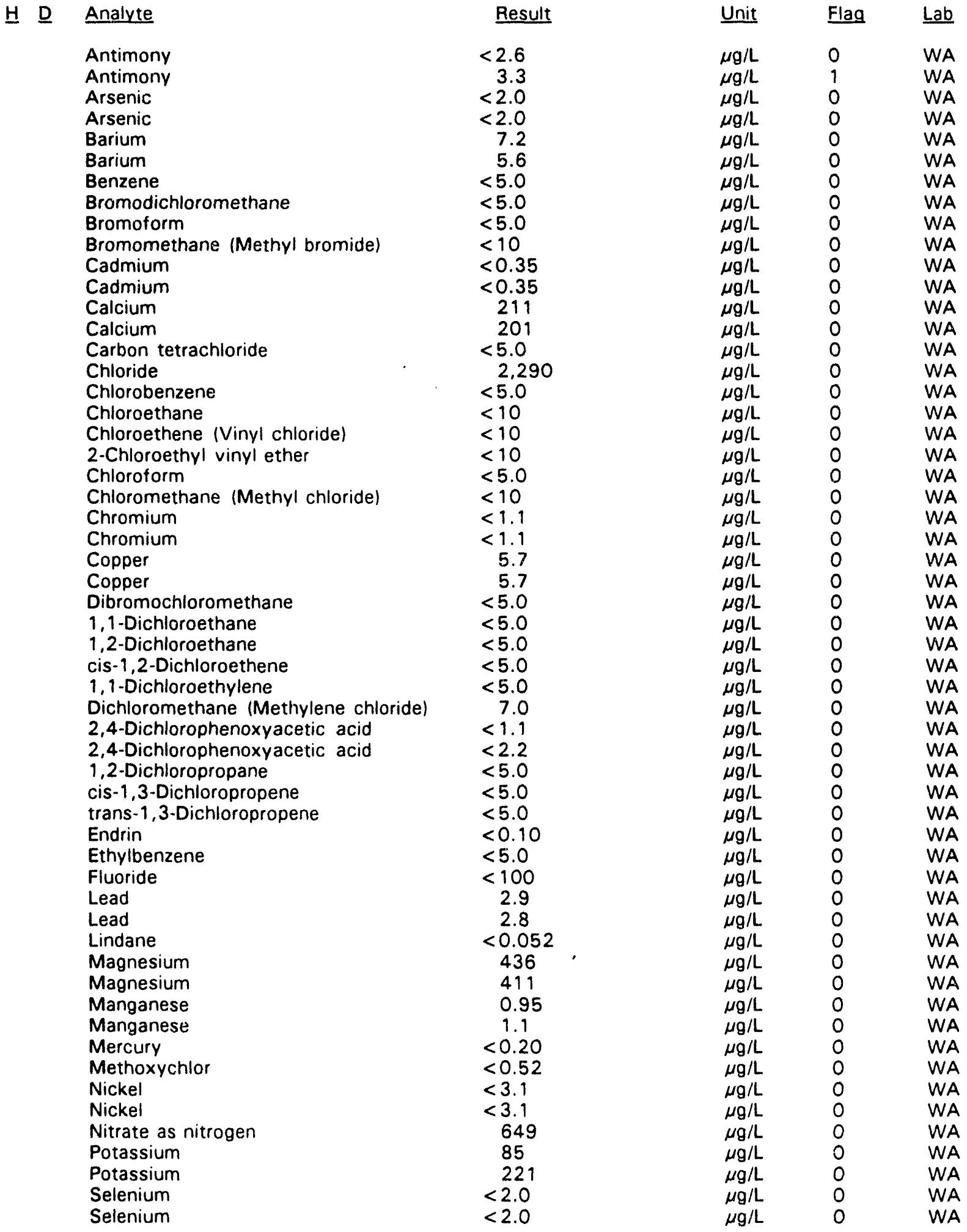

$\overline{-=}$ exceeded holding time. $\quad$ = exceeded primary drinking water standard. 
WELL LFW 25 collected on 06/02/92, laboratory analyses (cont.)

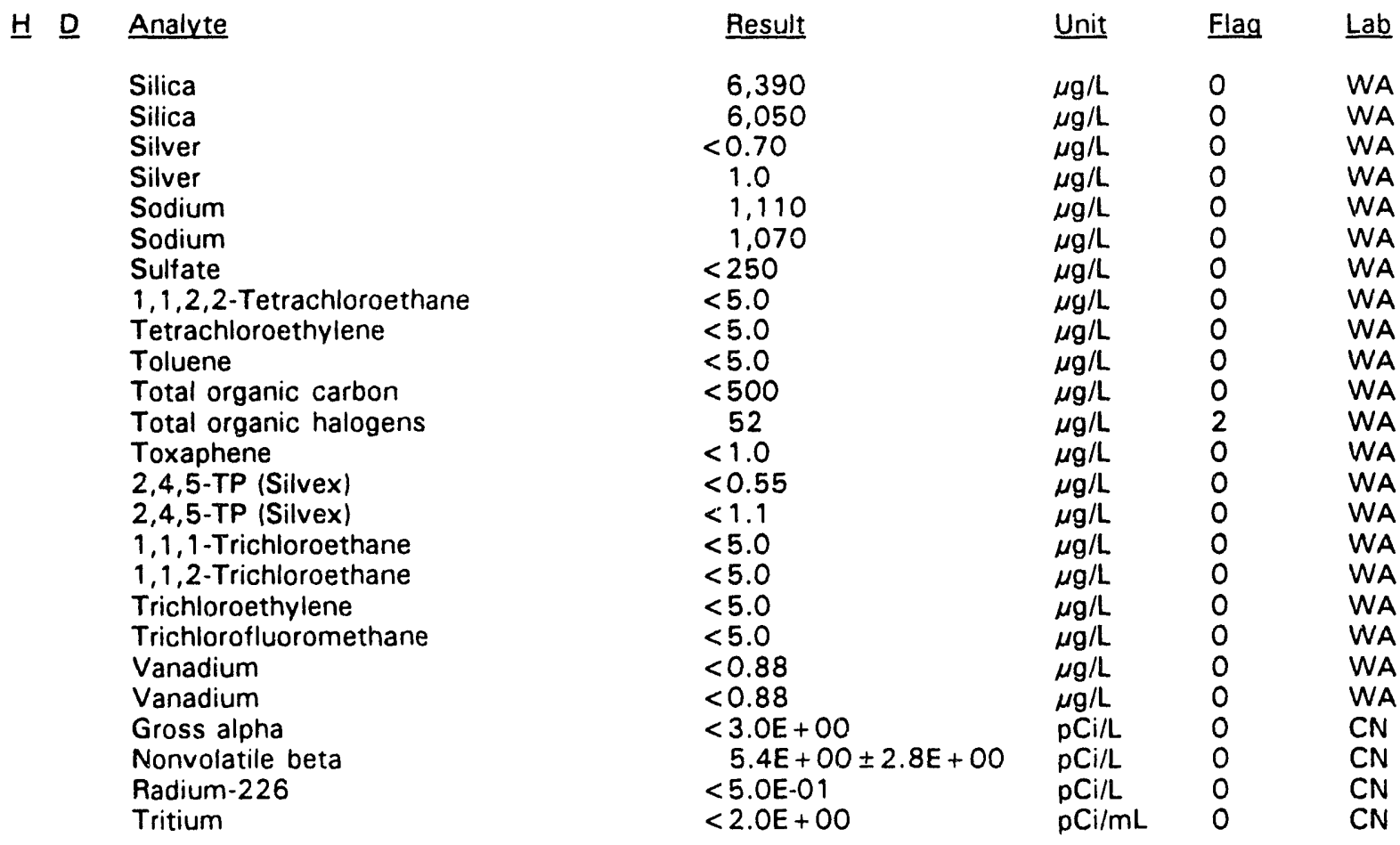

\section{WELL LFW 26}

$\begin{array}{llllll}\text { SRS Coord. } & \text { Lat/Longitude } & \text { Screen Zone Elevation } & \text { Top of Casing } & \text { Casing . Formation } \\ \text { N85654.6 } & 33.289663^{\circ} \mathrm{N} & 164.2-143.2 \mathrm{ft} \mathrm{msl} & 186.5 \mathrm{ft} \mathrm{msl} & \text { 4" PVC } \\ \text { E45633.8 } & 81.713023^{\circ} \mathrm{W} & & & \end{array}$

MEASUREMENTS CONDUCTED IN THE FIELD

Sample date: 06/02/92

Depth to water: $25.09 \mathrm{ft}(7.65 \mathrm{~m})$ below TOC

Water elevation: $161.41 \mathrm{ft}(49.20 \mathrm{~m}) \mathrm{ms}$

Sp. conductance: $13 \mu \mathrm{S} / \mathrm{cm}$

Water evacuated before sampling: $48 \mathrm{gal}$

\section{LABORATORY ANALYSES}

H $D$ Analyte

- $\mathrm{pH}$

- Specific conductance

Aluminum

Antimony

Arsenic

Barium

Benzene

Bromodichloromethane

Bromoform

$\begin{aligned} & \text { Result } \\ & 4.5 \\ 11 & \\ < & 15 \\ < & 2.6 \\ < & 2.0 \\ & 4.1 \\ < & 5.0 \\ < & 5.0 \\ < & 5.0\end{aligned}$

Time: $13: 25$

$\mathrm{pH}: 4.5$

Alkalinity: $0 \mathrm{mg} / \mathrm{L}$

Water temperature: $19.4^{\circ} \mathrm{C}$
Lab
WA
WA
WA
WA
WA
WA
WA
WA
WA
WA
WA
WA
WA
WA
WA
WA
WA
WA
WA
WA
WA
CN
CN
CN
CN 
WELL LFW 26 collected on 06/02/92, laboratory analyses (cont.)

\begin{tabular}{|c|c|c|c|c|c|}
\hline$\underline{H} \underline{D}$ & Analyte & Result & Unit & Flag & $\underline{L a b}$ \\
\hline & Bromomethane (Methyl bromide) & $<10$ & $\mu \mathrm{g} / \mathrm{L}$ & 0 & WA \\
\hline & Cadmium & $<0.35$ & $\mu g / L$ & 0 & WA \\
\hline & Calcium & 171 & $\mu \mathrm{g} / \mathrm{L}$ & 0 & WA \\
\hline & Carbon tetrachioride & $<5.0$ & $\mu \mathrm{g} / \mathrm{L}$ & 0 & WA \\
\hline & Chloride & 2,530 & $\mu g / L$ & 0 & WA \\
\hline & Chlorobenzene & $<5.0$ & $\mu g / L$ & 0 & WA \\
\hline & Chloroethane & $<10$ & $\mu \mathrm{g} / \mathrm{L}$ & 0 & WA \\
\hline & Chloroethene (Vinyl chloride) & $<10$ & $\mu \mathrm{g} / \mathrm{L}$ & 0 & WA \\
\hline & 2-Chloroethyl vinyl ether & $<10$ & $\mu \mathrm{g} / \mathrm{L}$ & 0 & WA \\
\hline & Chloroform & $<5.0$ & $\mu \mathrm{g} / \mathrm{L}$ & 0 & WA \\
\hline & Chloromethane (Methyl chloride) & $<10$ & $\mu g / L$ & 0 & WA \\
\hline & Chromium & $<1.1$ & $\mu \mathrm{g} / \mathrm{L}$ & 0 & WA \\
\hline & Copper & 2.0 & $\mu \mathrm{g} / \mathrm{L}$ & 0 & WA \\
\hline & Dibromochloromethane & $<5.0$ & $\mu \mathrm{g} / \mathrm{L}$ & 0 & WA \\
\hline & 1,1-Dichloroethane & $<5.0$ & $\mu g / L$ & 0 & WA \\
\hline & 1,2-Dichloroethane & $<5.0$ & $\mu \mathrm{g} / \mathrm{L}$ & 0 & WA \\
\hline & cis-1,2-Dichloroethene & $<5.0$ & $\mu \mathrm{g} / \mathrm{L}$ & 0 & WA \\
\hline & 1,1-Dichloroethylene & $<5.0$ & $\mu g / L$ & 0 & W:A \\
\hline & Dichloromethane (Methylene chloride) & 6.6 & $\mu g / L$ & 0 & WA \\
\hline & 2,4-Dichlorophenoxyacetic acid & $<1.1$ & $\mu \mathrm{g} / \mathrm{L}$ & 0 & WA \\
\hline & 1,2-Dichloropropane & $<5.0$ & $\mu g / L$ & 0 & WA \\
\hline & cis-1,3-Dichloropropene & $<5.0$ & $\mu \mathrm{g} / \mathrm{L}$ & 0 & WA \\
\hline & trans-1,3-Dichloropropene & $<5.0$ & $\mu \mathrm{g} / \mathrm{L}$ & 0 & WA \\
\hline & Endrin & $<0.11$ & $\mu g / L$ & 0 & WA \\
\hline & Endrin & $<0.22$ & $\mu \mathrm{g} / \mathrm{L}$ & 0 & WA \\
\hline & Ethylbenzene & $<5.0$ & $\mu \mathrm{g} / \mathrm{L}$ & 0 & WA \\
\hline & Fluoride & $<100$ & $\mu g / L$ & 0 & WA \\
\hline & Lead & $<2.0$ & $\mu g / L$ & 0 & WA \\
\hline & Lindane & $<0.054$ & $\mu \mathrm{g} / \mathrm{L}$ & 0 & WA \\
\hline & Lindane & $<0.11$ & $\mu g / L$ & 0 & WA \\
\hline & Magnesium & 238 & $\mu \mathrm{g} / \mathrm{L}$ & 0 & WA \\
\hline & Manganese & 1.1 & $\mu g / L$ & 0 & WA \\
\hline & Mercury & $<0.20$ & $\mu g / L$ & 0 & WA \\
\hline & Methoxychlor & $<0.54$ & $\mu \mathrm{g} / \mathrm{L}$ & 0 & WA \\
\hline & Methoxychlor & $<1.1$ & $\mu \mathrm{g} / \mathrm{L}$ & 0 & WA \\
\hline & Methoxychlor & $<1.1$ & $\mu \mathrm{g} / \mathrm{L}$ & 0 & WA \\
\hline & Nickel & $<3.1$ & $\mu g / L$ & 0 & WA \\
\hline & Nitrate as nitrogen & 352 & $\mu \mathrm{g} / \mathrm{L}$ & 0 & WA \\
\hline & Potassium & $<84$ & $\mu g / L$ & 0 & WA \\
\hline & Selenium & $<2.0$ & $\mu g / L$ & 0 & WA \\
\hline & Silica & 5,630 & $\mu \mathrm{g} / \mathrm{L}$ & 0 & WA \\
\hline & Silver & $<0.70$ & $\mu \mathrm{g} / \mathrm{L}$ & 0 & WA \\
\hline & Sodium & 1,010 & $\mu g / L$ & 0 & WA \\
\hline & Sulfate & $<250$ & $\mu g / L$ & 0 & WA \\
\hline & $1,1,2,2$-Tetrachloroethane & $<5.0$ & $\mu g / L$ & 0 & WA \\
\hline & Tetrachloroethylene & $<5.0$ & $\mu g / L$ & 0 & WA \\
\hline & Tolvene & $<5.0$ & $\mu \mathrm{g} / \mathrm{L}$ & 0 & WA \\
\hline & Total organic carbon & $<500$ & $\mu \mathrm{g} / \mathrm{L}$ & 0 & WA \\
\hline & Total organic halogens & $<20$ & $\mu g / L$ & 0 & WA \\
\hline & Total organic halogens & $<20$ & $\mu g / L$ & 0 & WA \\
\hline & Toxaphene & $<1.1$ & $\mu \mathrm{g} / \mathrm{L}$ & 0 & WA \\
\hline & Toxaphene & $<2.2$ & $\mu g / L$ & 0 & WA \\
\hline & Toxaphene & $<2.2$ & $\mu \mathrm{g} / \mathrm{L}$ & 0 & WA \\
\hline & 2,4,5-TP (Silvex) & $<0.55$ & $\mu \mathrm{g} / \mathrm{L}$ & 0 & WA \\
\hline & 1,1,1-Trichloroethane & $<5.0$ & $\mu \mathrm{g} / \mathrm{L}$ & 0 & WA \\
\hline & 1,1,2-Trichloroethane & $<5.0$ & $\mu \mathrm{g} / \mathrm{L}$ & 0 & WA \\
\hline
\end{tabular}

$\overline{- \text { = exceeded holding time. }}$ = exceeded primary drinking water standard. 
WELL LFW 26 collected on 06/02/92, laboratory analyses (cont.)

\begin{tabular}{|c|c|c|c|c|}
\hline \multirow[t]{8}{*}{$\underline{H} \underline{D}$} & Analyte & Result & Unit & Flag \\
\hline & Trichloroethylene & $<5.0$ & $\mu \mathrm{g} / \mathrm{L}$ & 0 \\
\hline & Trichlorofluoromethane & $<5.0$ & $\mu \mathrm{g} / \mathrm{L}$ & 0 \\
\hline & Vanadium & 1.4 & $\mu \mathrm{g} / \mathrm{L}$ & 0 \\
\hline & Gross alpha & $<3.0 E+00$ & $\mathrm{pCi} / \mathrm{L}$ & 0 \\
\hline & Nonvolatile beta & $<5.0 E+00$ & $\mathrm{pCi} / \mathrm{L}$ & 0 \\
\hline & Radium-226 & $5.9 \mathrm{E}-01 \pm 2.1 \mathrm{E}-01$ & $\mathrm{pCi} / \mathrm{L}$ & 0 \\
\hline & Tritium & $<2.0 E+00$ & $\mathrm{pCi} / \mathrm{mL}$ & 0 \\
\hline
\end{tabular}

\section{WELL LFW 27}

$\begin{array}{llllll}\text { SRS Coord. } & \text { Lat/Longitude } & \text { Screen Zone Elevation } & \text { Top of Casing } & \text { Casing } & \text { Formation } \\ \text { N85839.1 } & 33.290010^{\circ} \mathrm{N} & 163.9-142.9 \mathrm{ft} \mathrm{msl} & 189.2 \mathrm{ft} \mathrm{msl} & \text { 4" PVC } \\ \text { E45596.1 } & 81.713481^{\circ} \mathrm{W} & & & \end{array}$

MEASUREMENTS CONDUCTED IN THE FIELD

Sample date: 06/02/92

Depth to water: $26.77 \mathrm{ft}(8.16 \mathrm{~m})$ below TOC Water elevation: $162.43 \mathrm{ft}(49.51 \mathrm{~m}) \mathrm{msl}$ Sp. conductance: $14 \mu \mathrm{S} / \mathrm{cm}$

Water evacuated before sampling: $51 \mathrm{gal}$

\section{LABORATORY ANALYSES}

H $\underline{\text { Analyte }}$

$\bullet$

$\mathrm{pH}$
Specific conductance

Aluminum

- Antimony

Arsenic

Barium

Benzene

Bromodichloromethane

Bromoform

Bromomethane (Methyl bromide)

Cadmium

Calcium

Carbon tetrachloride

Chloride

Chlorobenzene

Chloroethane

Chloroethene (Vinyl chloride)

2-Chloroethyl vinyl ether

Chloroform

Chloromethane (Methyl chloride)

Chromium

Copper

Dibromochloromethane

1,1-Dichloroethane

1,2-Dichloroethane

cis-1,2-Dichloroethene

1,1-Dichloroethylene
Time: 12:55

pH: 4.6

Alkalinity: $0 \mathrm{mg} / \mathrm{L}$

Water temperature: $19.5^{\circ} \mathrm{C}$
$\underline{\operatorname{Lab}}$

WA

CN

$\mathrm{CN}$

$\mathrm{CN}$ 
WELL LFW 27 collected on 06/02/92, laboratory analyses (cont.)

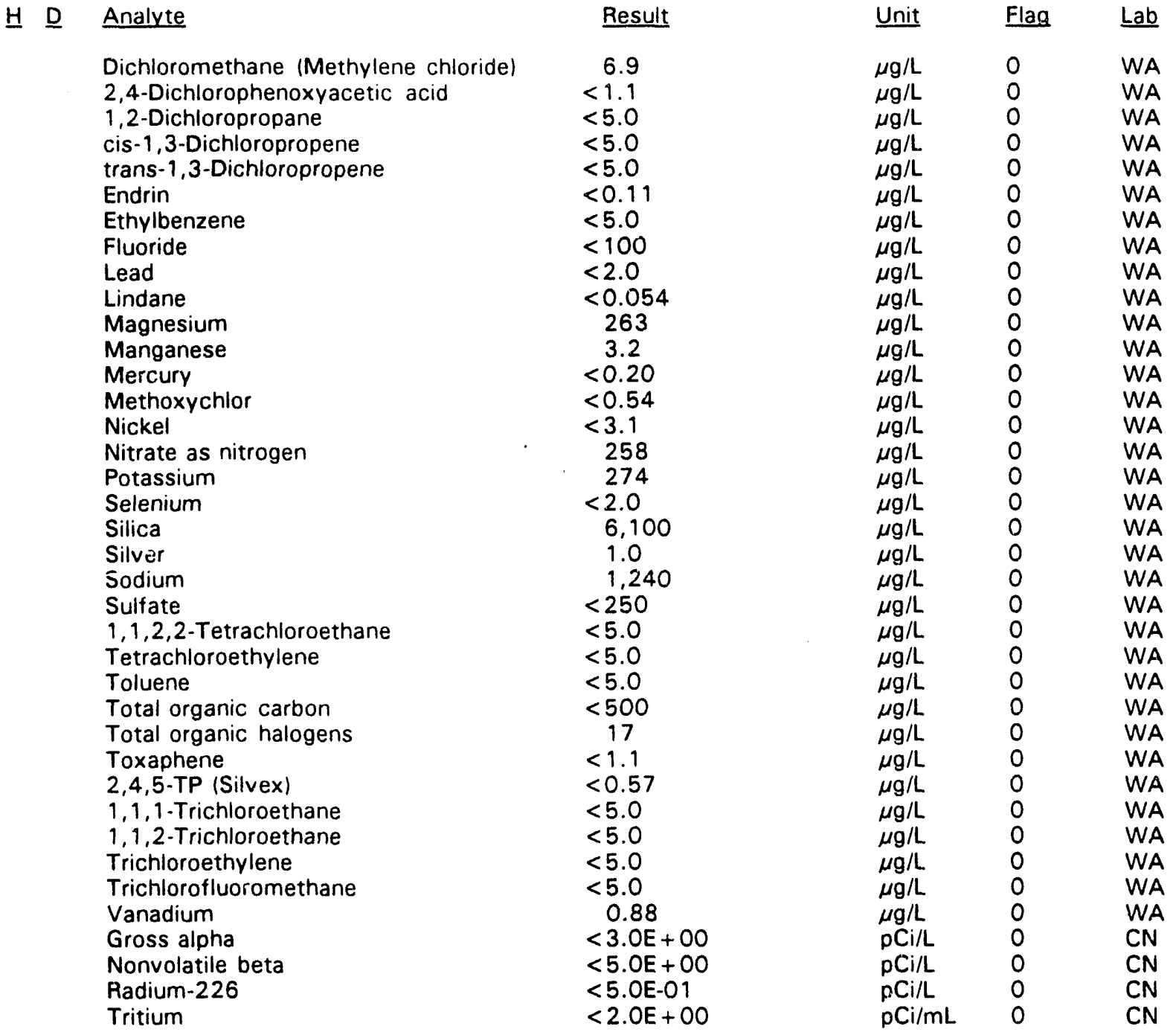

$\overline{- \text { = exceeded holding time. }} \mathbf{m}=$ exceeded primary drinking water standard. 
WELL LFW 28

$\begin{array}{llllll}\text { SRS Coord. } & \text { Lat/Longitude } & \text { Screen Zone Elevation } & \text { Top of Casing } & \text { Casing } & \text { Formation } \\ \text { N86079.6 } & 33.290475^{\circ} \mathrm{N} & 162.1-141.1 \mathrm{ft} \mathrm{msl} & 192.4 \mathrm{ft} \mathrm{msl} & \text { 4" PVC } \\ \text { E45555.3 } & 81.714056^{\circ} \mathrm{W} & & & \end{array}$

MEASUREMENTS CONDUCTED IN THE FIELD

Sample date: 06/05/92

Depth to water: $28.66 \mathrm{ft}(8.74 \mathrm{~m})$ below TOC

Water elevation: $163.74 \mathrm{ft}(49.91 \mathrm{~m} / \mathrm{msl}$

Sp. conductance: $28 \mu \mathrm{S} / \mathrm{cm}$

Water evacuated before sampling: $15 \mathrm{gal}$

The well went dry during purging.

Time: 9:15

$\mathrm{pH}: 4.3$

Alkalinity: $3 \mathrm{mg} / \mathrm{L}$

Water temperature: $19.8^{\circ} \mathrm{C}$

\section{LABORATORY ANALYSES}

$\vdots$

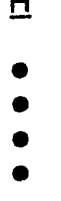

Analyte
pH
pH
Specific conductance
Specific conductance
Aluminum
Antimony
Arsenic
Barium
Benzene
Bromodichloromethane
Bromoform
Bromomethane (Methyl bromide)
Cadmium
Calcium
Carbon tetrachloride
Chloride
Chlorobenzene
Chloroethane
Chloroethene (Vinyl chloride)
2-Chloroethyl vinyl ether
Chloroform
Chloromethane (Methyl chloride)
Chromium
Copper
Dibromochloromethane
$1,1-$ Dichloroethane
$1,2-$ Dichloroethane
cis-1,2-Dichloroethene
$1,1-$ Dichloroethylene
Dichloromethane (Methylene chloride
2,4-Dichlorophenoxyacetic acid
1,2-Dichloropropane
cis-1,3-Dichloropropene
trans-1,3-Dichloropropene
Endrin
Ethylbenzene
Fluoride
Lead
Lindane

$\begin{aligned} & \text { Result } \\ & 5.6 \\ & 5.6 \\ & 24 \\ & 24 \\ & 15 \\ &< 2.6 \\ &< 2.0 \\ & 11 \\ &< 5.0 \\ &<5.0 \\ &<5.0 \\ &<10 \\ &<0.35 \\ & 2.000 \\ &<5.0 \\ & 2.090 \\ &<5.0 \\ &<10 \\ &<10 \\ &<10 \\ &<5.0 \\ &<10 \\ &<1.1 \\ &<11 \\ &<5.0 \\ &<5.0 \\ &<5.0 \\ &<5.0 \\ &<5.0 \\ & 1.8 \\ &< 1.1 \\ &<5.0 \\ &<5.0 \\ &<5.0 \\ &<0.11 \\ &<5.0 \\ &<100 \\ &<2.0 \\ &<0.057 \\ &<\end{aligned}$

\begin{tabular}{|c|c|c|}
\hline Unit & Flag & $\underline{L a b}$ \\
\hline $\mathrm{pH}$ & 0 & WA \\
\hline $\mathrm{pH}$ & 0 & WA \\
\hline$\mu \mathrm{S} / \mathrm{cm}$ & 0 & WA \\
\hline$\mu \mathrm{S} / \mathrm{cm}$ & 0 & WA \\
\hline$\mu g / L$ & 0 & WA \\
\hline$\mu \mathrm{g} / \mathrm{L}$ & 0 & WA \\
\hline$\mu \mathrm{g} / \mathrm{L}$ & 0 & WA \\
\hline$\mu \mathrm{g} / \mathrm{L}$ & 0 & WA \\
\hline$\mu \mathrm{g} / \mathrm{L}$ & 0 & WA \\
\hline$\mu \mathrm{g} / \mathrm{L}$ & 0 & WA \\
\hline$\mu \mathrm{g} / \mathrm{L}$ & 0 & WA \\
\hline$\mu \mathrm{g} / \mathrm{L}$ & 0 & WA \\
\hline$\mu \mathrm{g} / \mathrm{L}$ & 0 & WA \\
\hline$\mu \mathrm{g} / \mathrm{L}$ & .0 & WA \\
\hline$\mu \mathrm{g} / \mathrm{L}$ & 0 & WA \\
\hline$\mu \mathrm{g} / \mathrm{L}$ & 0 & WA \\
\hline$\mu \mathrm{g} / \mathrm{L}$ & 0 & WA \\
\hline$\mu \mathrm{g} / \mathrm{L}$ & 0 & WA \\
\hline$\mu \mathrm{g} / \mathrm{L}$ & 0 & WA \\
\hline$\mu \mathrm{g} / \mathrm{L}$ & 0 & WA \\
\hline$\mu \mathrm{g} / \mathrm{L}$ & 0 & WA \\
\hline$\mu \mathrm{g} / \mathrm{L}$ & 0 & WA \\
\hline$\mu \mathrm{g} / \mathrm{L}$ & 0 & WA \\
\hline$\mu \mathrm{g} / \mathrm{L}$ & 0 & WA \\
\hline$\mu \mathrm{g} / \mathrm{L}$ & 0 & WA \\
\hline$\mu g / L$ & 0 & WA \\
\hline$\mu g / L$ & 0 & WA \\
\hline$\mu \mathrm{g} / \mathrm{L}$ & 0 & WA \\
\hline$\mu \mathrm{g} / \mathrm{L}$ & 0 & WA \\
\hline$\mu \mathrm{g} / \mathrm{L}$ & 0 & WA \\
\hline$\mu \mathrm{g} / \mathrm{L}$ & 0 & WA \\
\hline$\mu \mathrm{g} / \mathrm{L}$ & 0 & WA \\
\hline$\mu \mathrm{g} / \mathrm{L}$ & 0 & WA \\
\hline$\mu \mathrm{g} / \mathrm{L}$ & 0 & WA \\
\hline$\mu \mathrm{g} / \mathrm{L}$ & 0 & WA \\
\hline$\mu \mathrm{g} / \mathrm{L}$ & 0 & WA \\
\hline$\mu \mathrm{g} / \mathrm{L}$ & 0 & WA \\
\hline$\mu \mathrm{g} / \mathrm{L}$ & 0 & WA \\
\hline$\mu g / L$ & 0 & WA \\
\hline
\end{tabular}

- = exceeded holding time. = exceeded primary drinking water standard. 
WELL LFW 28 collected on 06/05/92, laboratory analyses (cont.)

$\begin{array}{ll}\text { H. } & \text { Analyte } \\ \text { Magnesium } \\ \text { Manganese } \\ \text { Mercury } \\ \text { Methoxychlor } \\ \text { Nickel } \\ \text { Nitrate as nitrogen } \\ \text { Potassium } \\ \text { Selenium } \\ \text { Silica } \\ \text { Silver } \\ \text { Sodium } \\ \text { Suliate } \\ \text { Sulfate } \\ \text { 1,1,2,2-Tetrachloroethane } \\ \text { Tetrachloroethylene } \\ \text { Toluene } \\ \text { Total organic carbon } \\ \text { Total organic halogens } \\ \text { Toxaphene } \\ \text { 2,4,5-TP (Silvex) } \\ \text { 1,1,1-Trichloroethane } \\ \text { 1,1,2-Trichloroethane } \\ \text { Trichloroethylene } \\ \text { Trichlorofluoromethane } \\ \text { Vanadium } \\ \text { Gross alpha } \\ \text { Nonvolatile beta } \\ \text { Radium-226 } \\ \text { Tritium }\end{array}$

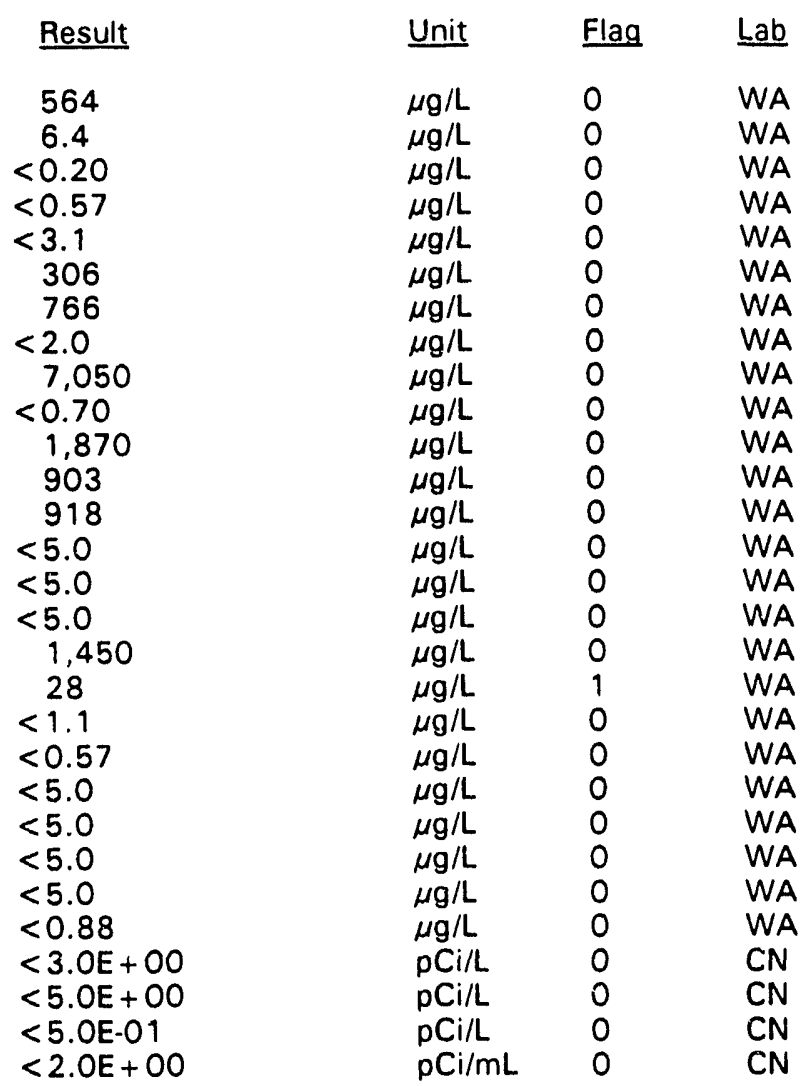

\section{WELL LFW 29}

\begin{tabular}{|c|c|c|c|c|c|}
\hline SRS Coord. & Lat/Longitude & Screen Zone Elevation & Top of Casing & Casing & Formation \\
\hline $\begin{array}{l}N 86372.7 \\
E 45503.3\end{array}$ & $\begin{array}{l}33.291038^{\circ} \mathrm{N} \\
81.714763^{\circ} \mathrm{W}\end{array}$ & $164.9-143.9 \mathrm{ft} \mathrm{msl}$ & 195 & /C & \\
\hline
\end{tabular}

MEASUREMENTS CONDUCTED IN THE FIELD

Sample date: 06/02/92

Depth to water: $30.04 \mathrm{ft}(9.16 \mathrm{~m})$ below TOC Water elevation: $165.26 \mathrm{ft}(50.37 \mathrm{~m}) \mathrm{msl}$ Sp. conductance: $22 \mu \mathrm{S} / \mathrm{cm}$.

Water evacuated before sampling: $56 \mathrm{gal}$

\section{LABORATORY ANALYSES}

Time: $12: 00$

$\mathrm{pH}: 4.3$

Alkalinity: $0 \mathrm{mg} / \mathrm{L}$

Water temperature: $18.6^{\circ} \mathrm{C}$

\begin{tabular}{|c|c|c|c|c|}
\hline$\underline{H} \underline{D}$ & Analyte & Result & $\underline{\text { Unit }}$ & Flag \\
\hline & $\begin{array}{l}\mathrm{pH} \\
\text { Specific conductance } \\
\text { Aluminum } \\
\text { Antininony } \\
\text { Arsenic }\end{array}$ & $\begin{aligned} & 4.4 \\
& 20 \\
& 18 \\
< & 2.6 \\
< & 2.0\end{aligned}$ & $\begin{array}{l}\mathrm{pH} \\
\mu \mathrm{S} / \mathrm{cm} \\
\mu \mathrm{g} / \mathrm{L} \\
\mu \mathrm{g} / \mathrm{L} \\
\mu \mathrm{g} / \mathrm{L}\end{array}$ & $\begin{array}{l}0 \\
0 \\
0 \\
0 \\
0\end{array}$ \\
\hline
\end{tabular}

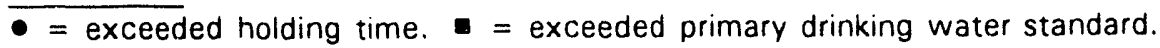


WELL LFW 29 collected on 06/02/92, laboratory analyses (cont.)

$\underline{H} \underline{D}$

Analyt

Barium

Benzene

Bromodichloromethane

Bromoform

Bromomethane (Methyl bromide)

Cadmium

Calcium

Carbon tetrachioride

Chloride

Chloride

Chlorobenzene

Chloroethane

Chloroethene (Vinyl chloride)

2-Chloroethyl vinyl ether

Chloroform

Chloromethane (Methyl chloride)

Chromium

Copper

Dibromochloromethane

1,1-Dichloroethane

1,2-Dichloroethane

cis-1,2-Dichloroethene

1,1-Dichloroethylene

Dichloromethane (Methylene chloride)

2,4-Dichlorophenoxyacetic acid

1,2-Dichloropropane

cis-1,3-Dichloropropene

trans-1,3-Dichloropropene

Endrin

Ethylbenzene

Fluoride

Lead

Lindane

Magnesium

Manganese

Mercury

Methoxychlor

Nickel

Nitrate as nitrogen

Potassium

Selenium

Silica

Silver

Sodium

Sulfate

1,1,2,2-Tetrachloroethane

Tetrachloroethylene

Tolvene

Total organic carbon

Total organic halogens

Toxaphene

2,4,5-TP (Silvex)

$1,1,1$-Trichloroethane

1,1,2-Trichloroethane

Trichloroethylene

Trichlorofluoromethane

\section{Result}

15

$<5.0$

$<5.0$

$<5.0$

$<10$

$<0.35$

482

$<5.0$

2,520

2,490

$<5.0$

$<10$

$<10$

$<10$

$<5.0$

$<10$

$<1.1$

5.5

$<5.0$

$<5.0$

$<5.0$

$<5.0$

$<5.0$

10

$<1.1$

$<5.0$

$<5.0$

$<5.0$

$<0.11$

$<5.0$

$<100$

$<2.0$

$<0.054$

480

3.1

$<0.20$

$<0.54$

$<3.1$

855

486

$<2.0$

5,320

$<0.70$

864

$<250$

$<5.0$

$<5.0$

$<5.0$

$<500$

30

$<1.1$

$<0.55$

$<5.0$

$<5.0$

$<5.0$

$<5.0$
Unit $\quad \underline{\text { Flag }} \quad \underline{\text { Lab }}$

$\mu g / L \quad 0 \quad$ WA

$\mu g / L \quad 0 \quad$ WA

$\mu g / L \quad 0 \quad$ WA

$\mu \mathrm{g} / \mathrm{L} \quad 0 \quad$ WA

$\mu g / L \quad 0 \quad$ WA

$\mu g / L \quad 0 \quad$ WA

$\mu g / L \quad$ WA

$\mu \mathrm{g} / \mathrm{L} \quad 0 \quad$ WA

$\mu g / L \quad 0 \quad$ WA

$\mu \mathrm{g} / \mathrm{L} \quad 0 \quad$ WA

$\mu g / L \quad 0 \quad$ WA

$\mu g / L \quad 0 \quad$ WA

$\mu g / L \quad$ WA

$\mu g / L \quad 0 \quad$ WA

$\mu g / L \quad$ WA

$\mu \mathrm{g} / \mathrm{L} \quad 0 \quad$ WA

$\mu g / L \quad 0 \quad$ WA

$\mu g / L \quad 0 \quad$ WA

$\begin{array}{lll}\mu / L & 0 & W A\end{array}$

$\mu g / L \quad 0 \quad$ WA

$\mu g / L \quad$ WA

$\mu g / L \quad$ WA

$\mu g / L \quad 0 \quad$ WA

$\mu g / L \quad 0 \quad$ WA

$\mu g / L \quad 0 \quad$ WA

$\mu \mathrm{g} / \mathrm{L} \quad 0 \quad$ WA

$\mu g / L \quad O \quad$ WA

$\mu \mathrm{g} / \mathrm{L} \quad 0 \quad W A$

$\mu g / L \quad O \quad W A$

$\mu g / L \quad 0 \quad$ WA

$\mu g / L \quad O \quad W A$

$\mu \mathrm{g} / \mathrm{L} \quad 0 \quad$ WA

$\mu g / L \quad 0 \quad$ WA

$\mu \mathrm{g} / \mathrm{L} \quad 0 \quad$ WA

$\mu g / L \quad 0 \quad$ WA

$\mu \mathrm{g} / \mathrm{L} \quad 0 \quad$ WA

$\mu g / L \quad 0 \quad$ WA

$\mu g / L \quad 0 \quad$ WA

$\mu g / L \quad W^{\mu} \quad 0 \quad$ WA

$\mu g / L \quad 0 \quad$ WA

$\mu g / L \quad 0 \quad W A$

$\mu g / L \quad 0 \quad$ WA

$\begin{array}{lll}\mu g / L & 0 & W A\end{array}$

$\mu g / L \quad 0 \quad$ WA

$\mu g / L \quad 0 \quad$ WA

$\mu g / L \quad 0 \quad$ WA

$\mu g / L \quad 0 \quad$ WA

$\mu g / L \quad 0 \quad$ WA

$\mu g / L \quad 0 \quad W A$

$\mu g / L \quad 1 \quad$ WA

$\mu g / L \quad 0 \quad$ WA

$\mu g / L \quad 0 \quad$ WA

$\mu g / L \quad 0 \quad W A$

$\mu g / L \quad 0 \quad$ WA

$\mu \mathrm{g} / \mathrm{L} \quad 0 \quad$ WA

$\mu g / L \quad 0 \quad$ WA

- exceeded holding time. = exceeded primary drinking water standard. 
WELL LFW 29 collected on 06/02/92, laboratory analyses (cont.)

\begin{tabular}{|c|c|c|c|}
\hline$\underline{H} \underline{D}$ Analyte & Result & Unit & Flag \\
\hline $\begin{array}{l}\text { Vanadium } \\
\text { Gross alpha } \\
\text { Nonvolatile beta } \\
\text { Radium-226 } \\
\text { Tritium }\end{array}$ & $\begin{array}{l}0.97 \\
<3.0 E+00 \\
7.0 E+00 \pm 2.9 E+00 \\
1.8 E+00 \pm 3.0 E-01 \\
<2.0 E+00\end{array}$ & $\begin{array}{l}\mu \mathrm{g} / \mathrm{L} \\
\mathrm{pCi} / \mathrm{L} \\
\mathrm{pCi} / \mathrm{L} \\
\mathrm{pCi} / \mathrm{L} \\
\mathrm{pCi} / \mathrm{mL}\end{array}$ & $\begin{array}{l}0 \\
0 \\
0 \\
0 \\
0\end{array}$ \\
\hline
\end{tabular}

\section{WELL LFW 30}

$\begin{array}{llllll}\text { SRS Coord. } & \text { Lat/Longitude } & \text { Screen Zone Elevation } & \text { Top of Casing } & \text { Casing } & \text { Formation } \\ \text { N86318.4 } & 33.290375^{\circ} \mathrm{N} & 162.7-141.7 \mathrm{ft} \mathrm{msl} & & 210.0 \mathrm{ft} \mathrm{msl} & \text { 4" PVC } \\ \text { E45170.9 } & 81.715532^{\circ} \mathrm{W} & & & \end{array}$

MEASUREMENTS CONDUCTED IN THE FIELD

Sample date: 06/02/92

Depth to water: $44.66 \mathrm{ft}(13.61 \mathrm{~m})$ below TOC

Water elevation: $165.34 \mathrm{ft}(50.40 \mathrm{~m}) \mathrm{msl}$

Sp. conductance: $22 \mu \mathrm{S} / \mathrm{cm}$

Water evacuated before sampling: 62 gal

\section{LABORATORY ANALYSES}

H $\underline{\text { Analyte }}$

$\begin{array}{lc}- \text { pH } & 4.9 \\ \text { Specific conductance } & 19 \\ \text { Aluminum } & 19 \\ \text { Antimony } & <2.6 \\ \text { Arsenic } & <2.0 \\ \text { Barium } & 15 \\ \text { Benzene } & <5.0 \\ \text { Bromodichloromethane } & <5.0 \\ \text { Bromoform } & <5.0 \\ \text { Bromomethane (Methyl bromide) } & <10 \\ \text { Cadmium } & <0.35 \\ \text { Calcium } & 1,480 \\ \text { Carbon tetrachloride } & <5.0 \\ \text { Ch'-ride } & 2,240 \\ \text { Chlorobenzene } & <5.0 \\ \text { Chloroethane } & <10 \\ \text { Chloroethene (Vinyl chloride) } & <10 \\ \text { 2-Chloroethyl vinyl ether } & <10 \\ \text { Chloroform } & <5.0 \\ \text { Chloromethane (Methyl chloride) } & <10 \\ \text { Chromium } & <1.1 \\ \text { Copper } & 5.0 \\ \text { Dibromochloromethane } & <5.0 \\ \text { 1,1-Dichloroethane } & <5.0 \\ \text { 1,2-Dichloroethane } & <5.0 \\ \text { cis-1,2-Dichloroethene } & <5.0 \\ \text { 1,1-Dichloroethylene } & <5.0 \\ \text { Dichloromethane (Methylene chloride) } & 4.4 \\ \text { 2,4-Dichlorophenoxyacetic acid } & <1.1 \\ & \end{array}$

Time: $11: 25$

pH: 4.7

Alkalinity: $1 \mathrm{mg} / \mathrm{L}$

Water temperature: $18.8^{\circ} \mathrm{C}$

\begin{tabular}{|c|c|c|}
\hline Unit & Flag & $\underline{\mathrm{Lab}}$ \\
\hline $\mathrm{pH}$ & 0 & WA \\
\hline$\mu \mathrm{S} / \mathrm{cm}$ & 0 & WA \\
\hline$\mu g / L$ & 0 & WA \\
\hline$\mu \mathrm{g} / \mathrm{L}$ & 0 & WA \\
\hline$\mu \mathrm{g} / \mathrm{L}$ & 0 & WA \\
\hline$\mu g / L$ & 0 & WA \\
\hline$\mu g / L$ & 0 & WA \\
\hline$\mu g / L$ & 0 & WA \\
\hline$\mu \mathrm{g} / \mathrm{L}$ & 0 & WA \\
\hline$\mu \mathrm{g} / \mathrm{L}$ & 0 & WA \\
\hline$\mu \mathrm{g} / \mathrm{L}$ & 0 & WA \\
\hline$\mu g / L$ & 0 & WA \\
\hline$\mu \mathrm{g} / \mathrm{L}$ & 0 & WA \\
\hline$\mu \mathrm{g} / \mathrm{L}$ & 0 & WA \\
\hline$\mu g / L$ & 0 & WA \\
\hline$\mu \mathrm{g} / \mathrm{L}$ & 0 & WA \\
\hline$\mu \mathrm{g} / \mathrm{L}$ & 0 & WA \\
\hline$\mu \mathrm{g} / \mathrm{L}$ & 0 & WA \\
\hline$\mu g / L$ & 0 & WA \\
\hline$\mu g / L$ & 0 & WA \\
\hline$\mu \mathrm{g} / \mathrm{L}$ & 0 & WA \\
\hline$\mu \mathrm{g} / \mathrm{L}$ & 0 & WA \\
\hline$\mu g / L$ & 0 & WA \\
\hline$\mu \mathrm{g} / \mathrm{L}$ & 0 & WA \\
\hline$\mu \mathrm{g} / \mathrm{L}$ & 0 & WA \\
\hline$\mu \mathrm{g} / \mathrm{L}$ & 0 & WA \\
\hline$\mu \mathrm{g} / \mathrm{L}$ & 0 & WA \\
\hline$\mu \mathrm{g} / \mathrm{L}$ & 0 & WA \\
\hline$\mu g / L$ & 0 & WA \\
\hline
\end{tabular}

$\overline{0}$ = exceeded holding time. $\mathbf{a}=$ exceeded primary drinking water standard. 
WELL LFW 30 collected on 06/02/92, laboratory analyses (cont.)

\section{H $\underline{\text { Analyte }}$}

1,2-Dichloropropane cis-1,3-Dichloropropene trans-1,3-Dichloropropene Endrin

Ethylbenzene

Fluoride

Lead

Lindane

Magnesium

Manganese

Mercury

Methoxychlor

Nickel

Nitrate as nitrogen

Potassium

Selenium

Silica

Silver

Sodium

Sulfate

1,1,2,2-Tetrachloroethane

Tetrachloroethylene

Toluene

Total organic carbon

Total organic halogens

Toxaphene

2,4,5-TP (Silvex)

1,1,1-Trichloroethane

1,1,2-Trichloroethane

Trichloroethylene

Trichlorofluoromethane

Vanadium

Gross alpha

Nonvolatile beta

Radium-226

Tritium

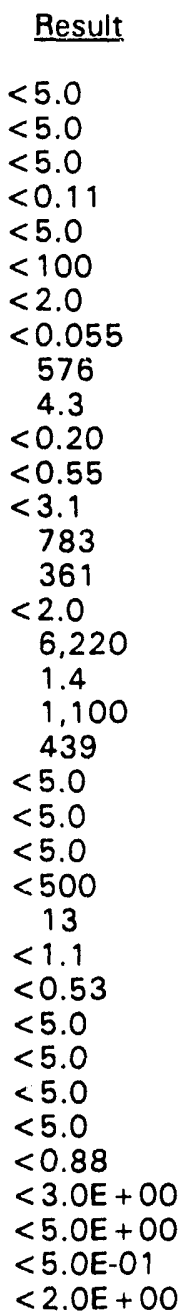

\begin{tabular}{|c|c|}
\hline Unit & Flag \\
\hline$\mu \mathrm{g} / \mathrm{L}$ & 0 \\
\hline$\mu \mathrm{g} / \mathrm{L}$ & 0 \\
\hline$\mu g / L$ & 0 \\
\hline$\mu \mathrm{g} / \mathrm{L}$ & 0 \\
\hline$\mu \mathrm{g} / \mathrm{L}$ & 0 \\
\hline$\mu \mathrm{g} / \mathrm{L}$ & 0 \\
\hline$\mu g / L$ & 0 \\
\hline$\mu \mathrm{g} / \mathrm{L}$ & 0 \\
\hline$\mu \mathrm{g} / \mathrm{L}$ & 0 \\
\hline$\mu g / L$ & 0 \\
\hline$\mu g / L$ & 0 \\
\hline$\mu g / L$ & 0 \\
\hline$\mu \mathrm{g} / \mathrm{L}$ & 0 \\
\hline$\mu g / L$ & 0 \\
\hline$\mu g / L$ & 0 \\
\hline$\mu \mathrm{g} / \mathrm{L}$ & 0 \\
\hline$\mu g / L$ & 0 \\
\hline$\mu \mathrm{g} / \mathrm{L}$ & 0 \\
\hline$\mu \mathrm{g} / \mathrm{L}$ & 0 \\
\hline$\mu \mathrm{g} / \mathrm{L}$ & 0 \\
\hline$\mu \mathrm{g} / \mathrm{L}$ & 0 \\
\hline$\mu g / L$ & 0 \\
\hline$\mu g / L$ & 0 \\
\hline$\mu \mathrm{g} / \mathrm{L}$ & 0 \\
\hline$\mu g / L$ & 0 \\
\hline$\mu g / L$ & 0 \\
\hline$\mu \mathrm{g} / \mathrm{L}$ & 0 \\
\hline$\mu \mathrm{g} / \mathrm{L}$ & 0 \\
\hline$\mu \mathrm{g} / \mathrm{L}$ & 0 \\
\hline$\mu \mathrm{g} / \mathrm{L}$ & 0 \\
\hline$\mu \mathrm{g} / \mathrm{L}$ & 0 \\
\hline$\mu g / L$ & 0 \\
\hline pCi/L & 0 \\
\hline pCi/L & 0 \\
\hline $\begin{array}{l}\mathrm{pCi} / \mathrm{L} \\
\mathrm{pCi} / \mathrm{mL}\end{array}$ & 0 \\
\hline
\end{tabular}

$\overline{- \text { exceeded holding time. }}$ = exceeded primary drinking water standard. 
WELL LFW 31

\begin{tabular}{|c|c|c|c|c|c|}
\hline SRS Coord. & Lat/Longitude & Screen Zone Elevation & Top of Casing & Casing & Formation \\
\hline $\begin{array}{l}\text { N86262.2 } \\
\text { E44869.0 }\end{array}$ & $\begin{array}{l}33.289758^{\circ} \mathrm{N} \\
81.716217^{\circ} \mathrm{W}\end{array}$ & $166.0-145.0 \mathrm{ft} \mathrm{msl}$ & $229.3 \mathrm{ft} \mathrm{msl}$ & $4^{n}$ PVC & \\
\hline
\end{tabular}

\section{MEASUREMENTS CONDUCTED IN THE FIELD}

Sample date: 06/02/92

Depth to water: $64.33 \mathrm{ft}(19.61 \mathrm{~m})$ below TOC

Water elevation: $164.97 \mathrm{ft}(50.28 \mathrm{~m}) \mathrm{msl}$

Sp. conductance: $22 \mu \mathrm{S} / \mathrm{cm}$

Water evacuated before sampling: $52 \mathrm{gal}$

\section{LABORATORY ANALYSES}

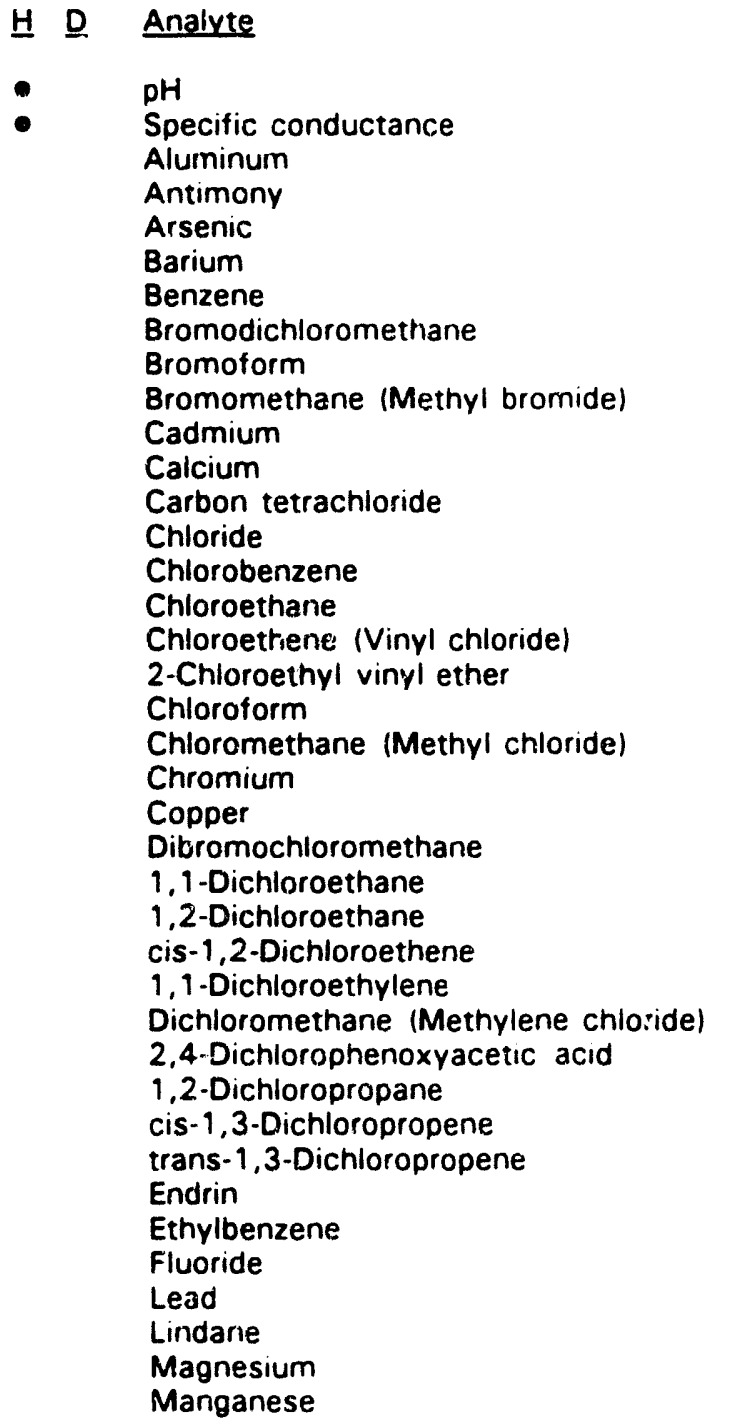

Time: $10: 50$

pH: 4.4

Alkalinity: $0 \mathrm{mg} / \mathrm{L}$

Water temperature: $18.3^{\circ} \mathrm{C}$

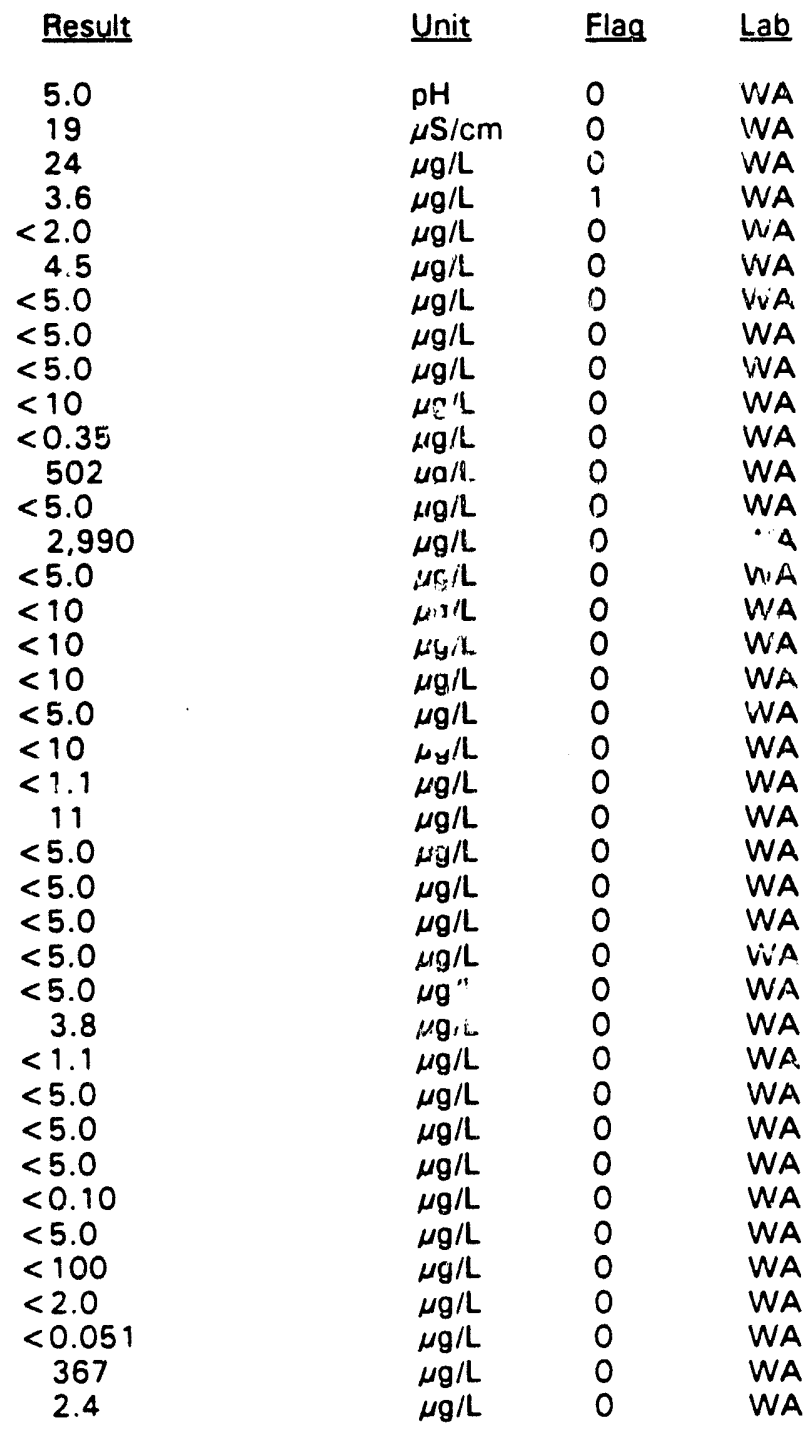

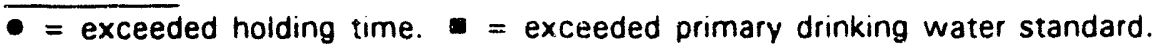


WELL LFW 31 collected on 06/02/92, laboratory analyses (cont.)

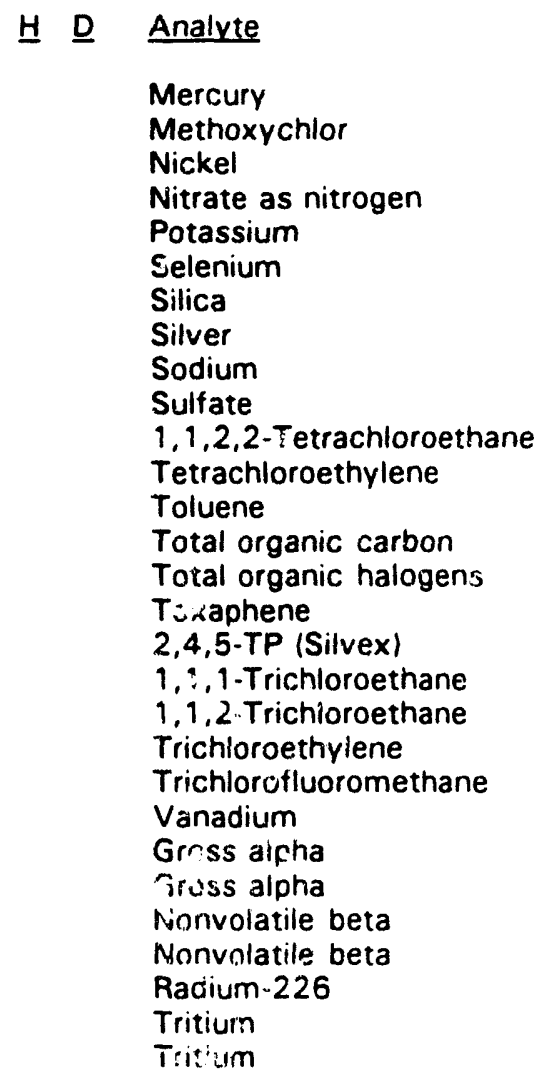

$\begin{aligned} & \text { Result } \\ &<0.20 \\ &< 0.51 \\ &< 3.1 \\ & 427 \\ & 720 \\ &< 2.0 \\ & 6.260 \\ & 1.6 \\ & 1.320 \\ & 894 \\ &< 5.0 \\ &< 5.0 \\ &<5.0 \\ &<500 \\ &< 10 \\ &< 1.0 \\ &<0.55 \\ &<5.0 \\ &<5.0 \\ &<5.0 \\ &<5.0 \\ &<0.88 \\ &<3.0 E+00 \\ &<3.0 E+00 \\ &<5.0 E+00 \\ &<5.0 E+00 \\ & 1.1 E+00 \pm 2.4 E-01 \\ & 2.1 E+00 \pm 4.1 E-01 \\ & 2.1 E+00 \pm 4.1 E-01\end{aligned}$

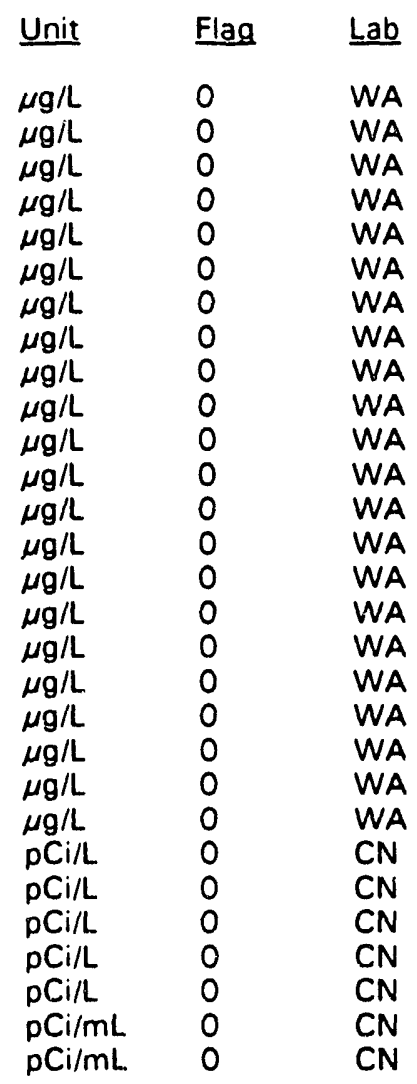

\section{WELL LFW 32}

$\begin{array}{llllll}\text { SRS Coord. } & \text { Lat/Longitude } & \text { Sc.een Zone Elevation } & \text { Top of Casing } & \text { Casing } & \text { Formation } \\ \text { N85836.8 } & 33.288927 \mathrm{vh} & 165.3-144.3 \mathrm{ft} \mathrm{msl} & 223.7 \mathrm{ft} \mathrm{msl} & 4^{\text {" PVC }} \\ \text { E44935.9 } & 81.715214^{\circ} \mathrm{W} & & & \end{array}$

MEASUREMENTS CONDUTTED IN THE FIELD

Sample date: 06/02/92

Depth to water: $60.35 \mathrm{ft}(18.39 \mathrm{~m})$ below TOC

Water elevation: $163.35 \mathrm{ft}(49.79 \mathrm{~m}) \mathrm{msl}$

Sp. conductance: $19 \mu \mathrm{S} / \mathrm{cm}$

Water evacuated before sampling: $50 \mathrm{gal}$

\section{LABORATORY ANALYSES}

므 Analyte

$\mathrm{pH}$

Specific conductance

Aluminum

Antimony

Arsenic
Time: 10:20

pH: 4.3

Alkalinity: $0 \mathrm{mg} / \mathrm{L}$

Water temperature: $18.4^{\circ} \mathrm{C}$
A

WA

WA

WA

A

WA

WA

WA

WA

WA

WA

WA

$\mathrm{CN}$

$\mathrm{CN}$

$\mathrm{CN}$

$\mathrm{CN}$

$\mathrm{CN}$ 
WELL LFW 32 collected on 06/02/92, laboratory analyses (cont.)

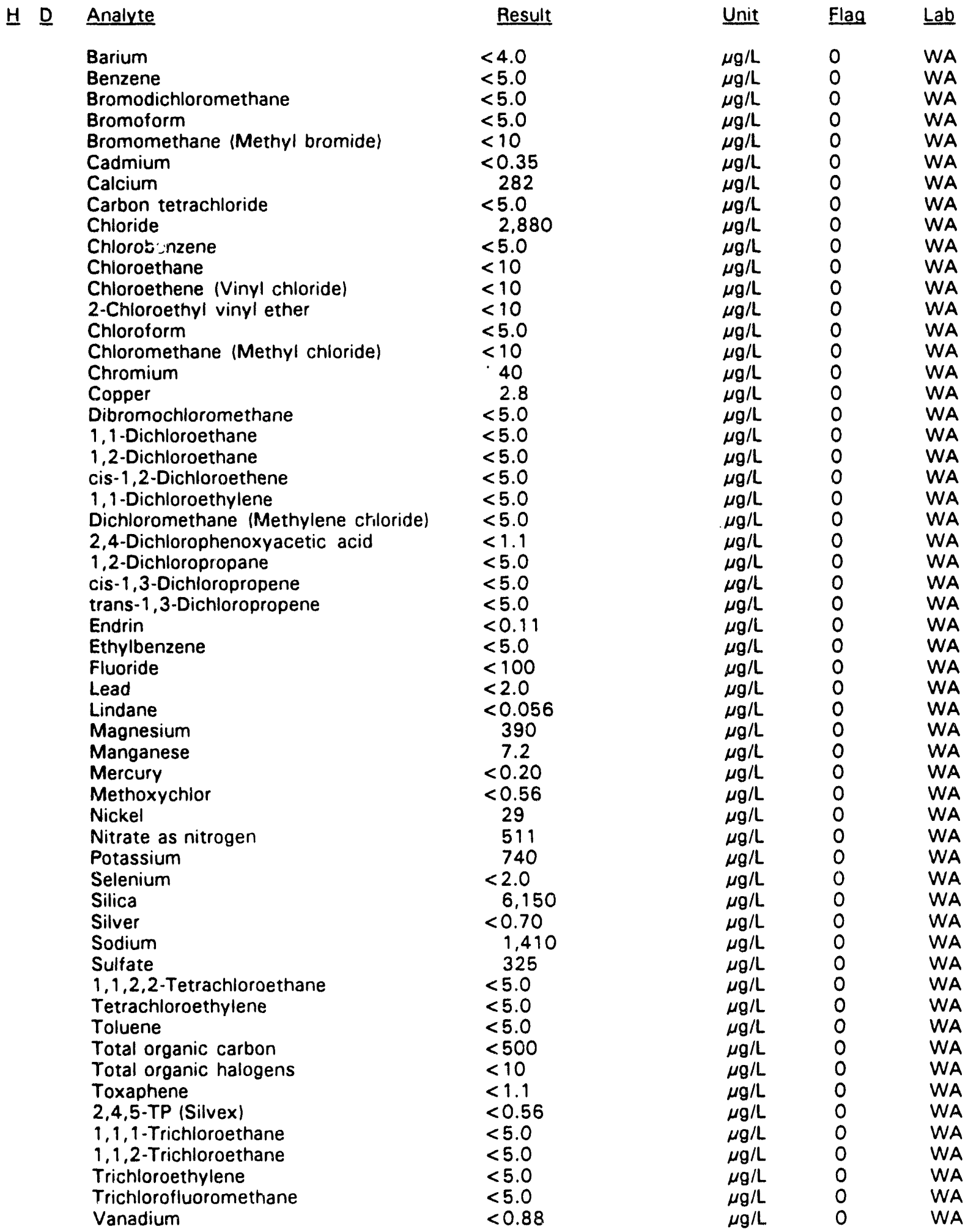

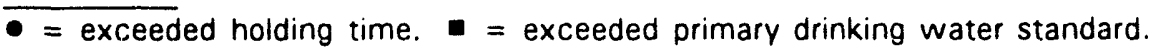


WELL LFW 32 collected on 06/02/92, laboratory analyses (cont.)

H $\underline{\text { Analyte }}$

Gross alpha

Nonvolatile beta

Radium-226

Tritium
Result

$$
\begin{aligned}
<3 . O E+00 \\
<5.0 E+00 \\
1.1 E+00 \pm 2.5 E-01 \\
\\
2.8 E+00 \pm 4.3 E-01
\end{aligned}
$$

$\underline{\text { Unit }} \quad \underline{\text { Flag }} \quad \underline{\text { Lab }}$

$\mathrm{pCi} / \mathrm{L} \quad \mathrm{C} \quad \mathrm{CN}$ $\mathrm{pCi} / \mathrm{L} \quad 0 \quad \mathrm{CN}$ $\mathrm{pCi} / \mathrm{L} \quad \mathrm{C} \quad \mathrm{CN}$ $\mathrm{pCi} / \mathrm{mL} \quad \mathrm{O} \quad \mathrm{CN}$

\section{WELL LFW 33}

$\begin{array}{llllll}\text { SRS Coord. } & \text { Lat/Longitude } & \text { Screen Zone Elevation } & \text { Top of Casing } & \text { Casing } & \text { Formation } \\ \text { N85633.8 } & \begin{array}{llll}33.288538^{\circ} \mathrm{N} \\ \text { E44973.0 }\end{array} & 81.714722^{\circ} \mathrm{W} & 165.4-144.4 \mathrm{ft} \mathrm{msl} & 213.7 \mathrm{ft} \mathrm{msl} & \text { 4" PVC }\end{array}$

MEASUREMENTS CONDUCTED IN THE FIELD

Sample date: 06/04/92

Depth to water: $52.58 \mathrm{ft}(16.03 \mathrm{~m})$ below TOC

Water elevation: $161.12 \mathrm{ft}(49.11 \mathrm{~m}) \mathrm{msl}$

Sp. conductance: $20 \mu \mathrm{S} / \mathrm{cm}$

Water evacuated before sampling: $44 \mathrm{gal}$

\section{LABORATORY ANALYSES}

H D Analyte

$\mathrm{pH}$

$\mathrm{pH}$

$\mathrm{pH}$

$\mathrm{pH}$

$\mathrm{pH}$

Specific conductance

Specific conductance

Specific conductance

Specific conductance

Specific conductance

Aluminum

Aluminum

Aluminum

Aluminum

Antimony

Antimony

Antimony

Antimony

Arsenic

Arsenic

Arsenic

Arsenic

Barium

Barium

Barium

Barium

Benzene

Benzene

Benzene

Benzene
Time: 9:20

pH: 4.3

Alkalinity: $0 \mathrm{mg} / \mathrm{L}$

Water temperature: $18.9^{\circ} \mathrm{C}$

\begin{tabular}{llll} 
Result & Unit & Flag & Lab \\
\hline 5.2 & $\mathrm{pH}$ & 0 & $\mathrm{GE}$ \\
5.2 & $\mathrm{pH}$ & 0 & $\mathrm{GE}$ \\
5.3 & $\mathrm{pH}$ & 0 & WA \\
5.3 & $\mathrm{pH}$ & 0 & WA \\
5.6 & $\mathrm{pH}$ & 0 & WA \\
20 & $\mu \mathrm{S} / \mathrm{cm}$ & 0 & $\mathrm{GE}$ \\
20 & $\mu \mathrm{S} / \mathrm{cm}$ & 0 & $\mathrm{GE}$ \\
17 & $\mu \mathrm{S} / \mathrm{cm}$ & 0 & WA \\
17 & $\mu \mathrm{S} / \mathrm{cm}$ & 0 & WA \\
16 & $\mu \mathrm{S} / \mathrm{cm}$ & 0 & WA \\
$<20$ & $\mu \mathrm{g} / \mathrm{L}$ & 0 & $\mathrm{GE}$ \\
$<20$ & $\mu \mathrm{g} / \mathrm{L}$ & 0 & $\mathrm{GE}$ \\
15 & $\mu \mathrm{g} / \mathrm{L}$ & 0 & WA \\
$<15$ & $\mu \mathrm{g} / \mathrm{L}$ & 0 & WA \\
$<2.0$ & $\mu \mathrm{g} / \mathrm{L}$ & 0 & $\mathrm{GE}$ \\
$<2.0$ & $\mu \mathrm{g} / \mathrm{L}$ & 0 & $\mathrm{GE}$ \\
$<2.6$ & $\mu \mathrm{g} / \mathrm{L}$ & 0 & WA \\
$<2.6$ & $\mu \mathrm{g} / \mathrm{L}$ & 0 & WA \\
$<2.0$ & $\mu \mathrm{g} / \mathrm{L}$ & 0 & $\mathrm{GE}$ \\
$<2.0$ & $\mu \mathrm{g} / \mathrm{L}$ & 0 & $\mathrm{GE}$ \\
$<2.0$ & $\mu \mathrm{g} / \mathrm{L}$ & 0 & WA \\
$<2.0$ & $\mu \mathrm{g} / \mathrm{L}$ & 0 & WA \\
$<3.0$ & $\mu \mathrm{g} / \mathrm{L}$ & 0 & $\mathrm{GE}$ \\
3.1 & $\mu \mathrm{g} / \mathrm{L}$ & 0 & GE \\
$<4.0$ & $\mu \mathrm{g} / \mathrm{L}$ & 0 & WA \\
$<4.0$ & $\mu \mathrm{g} / \mathrm{L}$ & 0 & WA \\
$<1.0$ & $\mu \mathrm{g} / \mathrm{L}$ & 0 & GE \\
$<1.0$ & $\mu \mathrm{g} / \mathrm{L}$ & 0 & $\mathrm{GE}$ \\
$<5.0$ & $\mu \mathrm{g} / \mathrm{L}$ & 0 & WA \\
$<5.0$ & $\mu \mathrm{g} / \mathrm{L}$ & 0 & WA \\
& & &
\end{tabular}

$\overline{-=\text { exceeded holding time. }} \cdot$ = exceeded primary drinking water standard. 
WELL LFW 33 collected on 06/04/92, laboratory analyses (cont.)

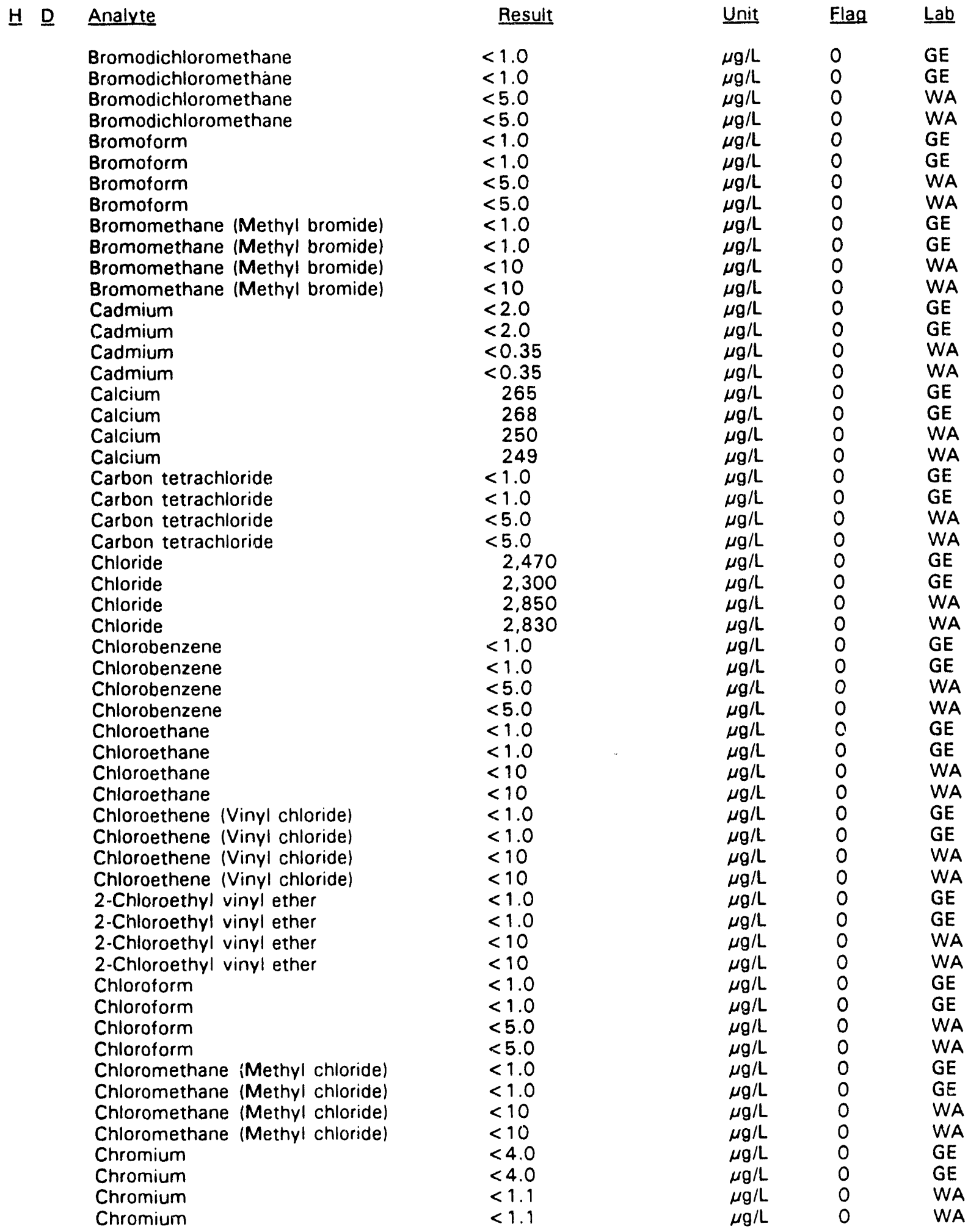

$\overline{- \text { = exceeded holding time. }} \mathbf{a}=$ exceeded primary drinking water standard. 
WELL LFW 33 collected on 06/04/92, laboratory analyses (cont.)

Analyte
Copper
Copper
Copper
Copper

Result

Unit Flag Lab

Dibromochloromethane

Dibromochloromethane

Dibromochloromethane

Dibromochloromethane

$<4.0$

$<4.0$

2.0

$<1.1$

$<1.0$

$<1.0$

1,1-Dichloroethane

$<5.0$

$<5.0$

1,1-Dichloroethane

1,1-Dichloroethane

1,1-Dichloroethane

$<1.0$

$<1.0$

1,2-Dichloroethane

$<5.0$

$<5.0$

1,2-Dichloroethane

$<1.0$

$<1.0$

1,2-Dichloroethane

$<5.0$

1,2-Dichloroethane

$<5.0$

cis-1,2-Dichloroethene

$<5.0$

cis-1,2-Dichloroethene

$<5.0$

1,1-Dichloroethylene

$<1.0$

1.1-Dichloroethylene

1,1-Dichloroethylene

$<1.0$

1,1-Dichloroethylene

$<5.0$

$<5.0$

trans-1,2-Dichloroethylene

$<1.0$

$<1.0$

Dichloromethane (Methylene chloride) $<1.0$

Dichloromethane (Methylene chloride) 4.1

Dichloromethane (Methylene chloride) 2.9

Dichloromethane (Methylene chloride) 2.3

2,4-Dichlorophenoxyacetic acid

$<0.30$

$<0.30$

$<1.1$

2,4-Dichlorophenoxyacetic acid

$<1.1$

$<1.0$

$<1.0$

$<5.0$

$<5.0$

$<1.0$

$<1.0$

$<5.0$

$<5.0$

$<1.0$

$<1.0$

$<5.0$

$<5.0$

$<0.0060$

$<0.0060$

$<0.0060$

$<0.11$

$<0.11$

$<1.0$

$<1.0$

$<5.0$

$<5.0$

$<100$

$<100$

$<100$

\begin{tabular}{|c|c|c|}
\hline$\mu g / L$ & 0 & GE \\
\hline$\mu \mathrm{g} / \mathrm{L}$ & 0 & $\mathrm{GE}$ \\
\hline$\mu \mathrm{g} / \mathrm{L}$ & 0 & WA \\
\hline$\mu \mathrm{g} / \mathrm{L}$ & 0 & WA \\
\hline$\mu g / L$ & 0 & GE \\
\hline$\mu \mathrm{g} / \mathrm{L}$ & 0 & GE \\
\hline$\mu \mathrm{g} / \mathrm{L}$ & 0 & WA \\
\hline$\mu g / L$ & 0 & WA \\
\hline$\mu \mathrm{g} / \mathrm{L}$ & 0 & $\mathrm{GE}$ \\
\hline$\mu \mathrm{g} / \mathrm{L}$ & 0 & $\mathrm{GE}$ \\
\hline$\mu \mathrm{g} / \mathrm{L}$ & 0 & WA \\
\hline$\mu g / L$ & 0 & WA \\
\hline$\mu \mathrm{g} / \mathrm{L}$ & 0 & GE \\
\hline$\mu \mathrm{g} / \mathrm{L}$ & 0 & GE \\
\hline$\mu \mathrm{g} / \mathrm{L}$ & 0 & WA \\
\hline$\mu \mathrm{g} / \mathrm{L}$ & 0 & WA \\
\hline$\mu \mathrm{g} / \mathrm{L}$ & 0 & WA \\
\hline$\mu \mathrm{g} / \mathrm{L}$ & 0 & WA \\
\hline$\mu g / L$ & 0 & $\mathrm{GE}$ \\
\hline$\mu \mathrm{g} / \mathrm{L}$ & 0 & $\mathrm{GE}$ \\
\hline$\mu \mathrm{g} / \mathrm{L}$ & 0 & WA \\
\hline$\mu \mathrm{g} / \mathrm{L}$ & 0 & WA \\
\hline$\mu \mathrm{g} / \mathrm{L}$ & 0 & GE \\
\hline$\mu \mathrm{g} / \mathrm{L}$ & 0 & GE \\
\hline$\mu \mathrm{g} / \mathrm{L}$ & 0 & GE \\
\hline$\mu \mathrm{g} / \mathrm{L}$ & 0 & GE \\
\hline$\mu \mathrm{g} / \mathrm{L}$ & 0 & WA \\
\hline$\mu \mathrm{g} / \mathrm{L}$ & 0 & WA \\
\hline$\mu g / L$ & 0 & GE \\
\hline$\mu \mathrm{g} / \mathrm{L}$ & 0 & GE \\
\hline$\mu \mathrm{g} / \mathrm{L}$ & 0 & WA \\
\hline$\mu \mathrm{g} / \mathrm{L}$ & 0 & WA \\
\hline$\mu g / L$ & 0 & GE \\
\hline$\mu \mathrm{g} / \mathrm{L}$ & 0 & GE \\
\hline$\mu \mathrm{g} / \mathrm{L}$ & 0 & WA \\
\hline$\mu \mathrm{g} / \mathrm{L}$ & 0 & WA \\
\hline$\mu \mathrm{g} / \mathrm{L}$ & 0 & GE \\
\hline$\mu \mathrm{g} / \mathrm{L}$ & 0 & GE \\
\hline$\mu \mathrm{g} / \mathrm{L}$ & 0 & WA \\
\hline$\mu \mathrm{g} / \mathrm{L}$ & 0 & WA \\
\hline$\mu \mathrm{g} / \mathrm{L}$ & 0 & GE \\
\hline$\mu \mathrm{g} / \mathrm{L}$ & 0 & GE \\
\hline$\mu \mathrm{g} / \mathrm{L}$ & 0 & WA \\
\hline$\mu \mathrm{g} / \mathrm{L}$ & 0 & WA \\
\hline$\mu g / L$ & 0 & GE \\
\hline$\mu g / L$ & 0 & GE \\
\hline$\mu \mathrm{g} / \mathrm{L}$ & 0 & GE \\
\hline$\mu g / \mathrm{L}$ & 0 & WA \\
\hline$\mu \mathrm{g} / \mathrm{L}$ & 0 & WA \\
\hline$\mu g / L$ & 0 & GE \\
\hline$\mu \mathrm{g} / \mathrm{L}$ & 0 & $\mathrm{GE}$ \\
\hline$\mu \mathrm{g} / \mathrm{L}$ & 0 & WA \\
\hline$\mu \mathrm{g} / \mathrm{L}$ & 0 & WA \\
\hline$\mu \mathrm{g} / \mathrm{L}$ & 0 & $\mathrm{GE}$ \\
\hline$\mu g / L$ & 0 & $\mathrm{GE}$ \\
\hline$\mu \mathrm{g} / \mathrm{L}$ & 0 & W \\
\hline
\end{tabular}

$\overline{- \text { = exceeded holding time. }} \mathbf{\square}=$ exceeded primary drinking water standard. 
WELL LFW 33 collected on 06/04/92, laboratory analyses (cont.)

\begin{tabular}{|c|c|c|c|c|c|}
\hline$\underline{H} \quad \underline{D}$ & Analyte & Result & Unit & Flag & Lab \\
\hline & Fluoride & $<100$ & $\mu \mathrm{g} / \mathrm{L}$ & 0 & WA \\
\hline & Lead & $<3.0$ & $\mu \mathrm{g} / \mathrm{L}$ & 0 & GE \\
\hline & Lead & $<3.0$ & $\mu \mathrm{g} / \mathrm{L}$ & 0 & $\mathrm{GE}$ \\
\hline & Lead & $<2.0$ & $\mu \mathrm{g} / \mathrm{L}$ & 0 & WA \\
\hline & Lead & $<2.0$ & $\mu g / L$ & 0 & WA \\
\hline & Lindane & $<0.0050$ & $\mu \mathrm{g} / \mathrm{L}$ & 0 & GE \\
\hline & Lindane & $<0.0050$ & $\mu g / L$ & 0 & $\mathrm{GE}$ \\
\hline & Lindane & $<0.0050$ & $\mu g / L$ & $D$ & GE \\
\hline & Lindane & $<0.054$ & $\mu g / L$ & 0 & WA \\
\hline & Lindane & $<0.055$ & $\mu g / L$ & 0 & WA \\
\hline & Magnesium & 371 & $\mu g / L$ & 0 & $\mathrm{GE}$ \\
\hline & Magnesium & 376 & $\mu g / L$ & 0 & GE \\
\hline & Magnesium & 342 & $\mu g / L$ & 0 & WA \\
\hline & Magnesium & 337 & $\mu g / L$ & 0 & WA \\
\hline & Manganese & $<2.0$ & $\mu g / L$ & 0 & GE \\
\hline & Manganese & $<2.0$ & $\mu \mathrm{g} / \mathrm{L}$ & 0 & GE \\
\hline & Manganese & 0.85 & $\mu \mathrm{g} / \mathrm{L}$ & 0 & WA \\
\hline & Manganese & 0.67 & $\mu g / L$ & 0 & WA \\
\hline & Mercury & $<0.20$ & $\mu \mathrm{g} / \mathrm{L}$ & 0 & GE \\
\hline & Mercury & $<0.20$ & $\mu \mathrm{g} / \mathrm{L}$ & 0 & GE \\
\hline & Mercury & $<0.20$ & $\mu \mathrm{g} / \mathrm{L}$ & 0 & WA \\
\hline & Mercury & $<0.20$ & $\mu g / L$ & 0 & WA \\
\hline & Methoxychlor & $<0.50$ & $\mu g / L$ & 0 & GE \\
\hline & Methoxychlor & $<0.50$ & $\mu g / L$ & 0 & GE \\
\hline & Methoxychlor & $<0.50$ & $\mu g / L$ & 0 & GE \\
\hline & Methoxychlor & $<0.54$ & $\mu \mathrm{g} / \mathrm{L}$ & 0 & WA \\
\hline & Methoxychlor & $<0.55$ & $\mu \mathrm{g} / \mathrm{L}$ & 0 & WA \\
\hline & Nickel & $<4.0$ & $\mu \mathrm{g} / \mathrm{L}$ & 0 & GE \\
\hline & Nickel & $<4.0$ & $\mu \mathrm{g} / \mathrm{L}$ & 0 & GE \\
\hline & Nickel & $<3.1$ & $\mu \mathrm{g} / \mathrm{L}$ & 0 & WA \\
\hline & Nickel & $<3.1$ & $\mu \mathrm{g} / \mathrm{L}$ & 0 & WA \\
\hline & Nitrate as nitrogen & 460 & $\mu g / L$ & 0 & GE \\
\hline & Nitrate as nitrogen & 490 & $\mu g / L$ & 0 & GE \\
\hline & Nitrate as nitrogen & 500 & $\mu g / L$ & 0 & GE \\
\hline & Nitrate as nitrogen & 473 & $\mu g / L$ & 0 & WA \\
\hline & Nitrate as nitrogen & 415 & $\mu \mathrm{g} / \mathrm{L}$ & 0 & WA \\
\hline & Potassium & 583 & $\mu g / L$ & 0 & GE \\
\hline & Potassium & 642 & $\mu g / L$ & 0 & GE \\
\hline & Potassium & 567 & $\mu \mathrm{g} / \mathrm{L}$ & 0 & WA \\
\hline & Potassium & 586 & $\mu \mathrm{g} / \mathrm{L}$ & 0 & WA \\
\hline & Selenium & $<2.0$ & $\mu g / L$ & 0 & GE \\
\hline & Selenium & $<2.0$ & $\mu \mathrm{g} / \mathrm{L}$ & 0 & GE \\
\hline & Selenium & $<2.0$ & $\mu \mathrm{g} / \mathrm{L}$ & 0 & WA \\
\hline & Selenium & $<2.0$ & $\mu \mathrm{g} / \mathrm{L}$ & 0 & WA \\
\hline & Silica & 6,370 & $\mu g / L$ & 0 & $\mathrm{GE}$ \\
\hline & Silica & 6,450 & $\mu g / L$ & 0 & GE \\
\hline & Silica & 6.050 & $\mu g / L$ & 0 & WA \\
\hline & Silica & 6.140 & $\mu g / L$ & 0 & WA \\
\hline & Silver & $<2.0$ & $\mu g / L$ & 0 & GE \\
\hline & Silver & $<2.0$ & $\mu \mathrm{g} / \mathrm{L}$ & 0 & $\mathrm{GE}$ \\
\hline & Silver & 1.0 & $\mu \mathrm{g} / \mathrm{L}$ & 0 & WA \\
\hline & Silver & $<0.70$ & $\mu g / L$ & 0 & WA \\
\hline & Sodium & 1,330 & $\mu g / L$ & 0 & GE \\
\hline & Sodium & 1,360 & $\mu g / L$ & 0 & GE \\
\hline & Sodium & 1,370 & $\mu g / L$ & 0 & WA \\
\hline & Sodium & 1,390 & $\mu g / L$ & 0 & WA \\
\hline
\end{tabular}

$\overline{-=\text { exceeded holding time. }}$ - = exceeded primary drinking water standard. 
WELL LFW 33 collected on 06/04/92, laboratory analyses (cont.)

\begin{tabular}{|c|c|c|c|c|}
\hline$\underline{H} \underline{D} \quad$ Analyte & Result & $\underline{\text { Unit }}$ & Flag & $\underline{L a b}$ \\
\hline Sulfate & $<1,000$ & $\mu \mathrm{g} / \mathrm{L}$ & 0 & GE \\
\hline Sulfate & $<1,000$ & $\mu \mathrm{g} / \mathrm{L}$ & 0 & GE \\
\hline Sulfate & 402 & $\mu \mathrm{g} / \mathrm{L}$ & 0 & WA \\
\hline Sulfate & 377 & $\mu \mathrm{g} / \mathrm{L}$ & 0 & WA \\
\hline $1,1,2,2$-Tetrachloroethane & $<1.0$ & $\mu g / L$ & 0 & GE \\
\hline $1,1,2,2$-Tetrachloroethane & $<1.0$ & $\mu \mathrm{g} / \mathrm{L}$ & 0 & GE \\
\hline $1,1,2,2$-Tetrachloroethane & $<5.0$ & $\mu g / L$ & 0 & WA \\
\hline $1,1,2,2$-Tetrachloroethane & $<5.0$ & $\mu g / L$ & 0 & WA \\
\hline Tetrachloroethylene & $<1.0$ & $\mu \mathrm{g} / \mathrm{L}$ & 0 & GE \\
\hline Tetrachloroethylene & $<1.0$ & $\mu g / L$ & 0 & GE \\
\hline Tetrachloroethylene & $<5.0$ & $\mu g / L$ & 0 & WA \\
\hline Tetrachloroethylene & $<5.0$ & $\mu g / L$ & 0 & WA \\
\hline Toluene & $<1.0$ & $\mu g / L$ & 0 & $\mathrm{GE}$ \\
\hline Toluene & $<1.0$ & $\mu \mathrm{g} / \mathrm{L}$ & 0 & GE \\
\hline Toluene & $<5.0$ & $\mu \mathrm{g} / \mathrm{L}$ & 0 & WA \\
\hline Toluene & $<5.0$ & $\mu \mathrm{g} / \mathrm{L}$ & 0 & WA \\
\hline Total organic carbon & $<1,000$ & $\mu g / L$ & 0 & GE \\
\hline Total organic carbon & $<1,000$ & $\mu \mathrm{g} / \mathrm{L}$ & 0 & GE \\
\hline Total organic carbon & 669 & $\mu g / L$ & 0 & WA \\
\hline Total organic carbon & 66.9 & $\mu g / L$ & 0 & WA \\
\hline Total organic halogens & $<5.0$ & $\mu g / L$ & 0 & GE \\
\hline Total organic halogens & $<5.0$ & $\mu g / L$ & 0 & GE \\
\hline Total organic halogens & 28 & $\mu \mathrm{g} / \mathrm{L}$ & 1 & WA \\
\hline Total organic halogens & 25 & $\mu \mathrm{g} / \mathrm{L}$ & 0 & Wh \\
\hline Total organic halogens & 182 & $\mu \mathrm{g} / \mathrm{L}$ & 2 & WA \\
\hline Toxaphene & $<0.24$ & $\mu \mathrm{g} / \mathrm{L}$ & $\overline{0}$ & GE \\
\hline Toxaphene & $<0.24$ & $\mu \mathrm{g} / \mathrm{L}$ & 0 & GE \\
\hline Toxaphene & $<0.24$ & $\mu g / L$ & 0 & GE \\
\hline Toxaphene & $<1.1$ & $\mu g / L$ & 0 & NA \\
\hline Toxaphene & $<1.1$ & $\mu g / L$ & 0 & WA \\
\hline 2,4,5-TP (Silvex) & $<0.090$ & $\mu g / L$ & 0 & jE \\
\hline 2,4,5-TP (Silvex) & $<0.090$ & $\mu g / L$ & 0 & 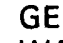 \\
\hline 2,4,5-TP (Silvex) & $<0.56$ & $\mu g / L$ & 0 & Wh \\
\hline 2,4,5-TP (Silvex) & $<0.56$ & $\mu \mathrm{g} / \mathrm{L}$ & 0 & Wh \\
\hline 1,1,1-Trichloroethane & $<1.0$ & $\mu \mathrm{g} / \mathrm{L}$ & 0 & $\mathrm{GE}$ \\
\hline $1,1,1$-Trichloroethane & $<1.0$ & $\mu \mathrm{g} / \mathrm{L}$ & 0 & $\overrightarrow{G E}$ \\
\hline 1,1,1-Trichloroethane & $<5.0$ & $\mu g / L$ & 0 & WA \\
\hline 1,1,1-Trichloroethane & $<5.0$ & $\mu g / L$ & 0 & Wr \\
\hline 1,1,2-Trichloroethane & $<1.0$ & $\mu g / L$ & 0 & $\mathrm{jE}$ \\
\hline 1,1,2-Trichloroethane & $<1.0$ & $\mu \mathrm{g} / \mathrm{L}$ & 0 & JE \\
\hline $1,1,2$-Trichloroethane & $<5.0$ & $\mu \mathrm{g} / \mathrm{L}$ & 0 & W \\
\hline 1,1,2-Trichloroethane & $<5.0$ & $\mu g / L$ & 0 & N/ \\
\hline Trichloroethylene & $<1.0$ & $\mu \mathrm{g} / \mathrm{L}$ & 0 & $E$ \\
\hline Trichloroethylene & $<1.0$ & $\mu \mathrm{g} / \mathrm{L}$ & 0 & \\
\hline Trichloroethylene & $<5.0$ & $\mu \mathrm{g} / \mathrm{L}$ & 0 & Wt \\
\hline Trichloroethylene & $<5.0$ & $\mu \mathrm{g} / \mathrm{L}$ & 0 & W \\
\hline Trichlorofluoromethane & 10 & $\mu \mathrm{g} / \mathrm{L}$ & 2 & $E$ \\
\hline Trichlorofluoromethane & $<1.0$ & $\mu \mathrm{g} / \mathrm{L}$ & 0 & \\
\hline Trichlorofluoromethane & $<5.0$ & $\mu \mathrm{g} / \mathrm{L}$ & 0 & \\
\hline Trichlorofluoromethane & $<5.0$ & $\mu \mathrm{g} / \mathrm{L}$ & 0 & \\
\hline Vanadium & $<8.0$ & $\mu g / L$ & 0 & \\
\hline Vanadium & $<8.0$ & $\mu \mathrm{g} / \mathrm{L}$ & 0 & \\
\hline Vanadium & 0.88 & $\mu \mathrm{g} / \mathrm{L}$ & 0 & \\
\hline Vanadium & $<0.88$ & $\mu g / L$ & 0 & \\
\hline Gross alpha & $6.8 E+00 \pm 1.9 E+00$ & $\mathrm{pCi} / \mathrm{L}$ & 0 & \\
\hline Gross alpha & $7.6 E+00 \pm 1.0 E+00$ & $\mathrm{pCi} / \mathrm{L}$ & 1 & \\
\hline
\end{tabular}

- = exceeded holding time. = exceeded primary drinking water standard. 
WELL LFW 33 collected on 06/04/92, laboratory analyses (cont.)

H D Analyte

Gross alpha
Gross alpha
Nonvolatile beta
Nonvolatile beta
Nonvolatile beta
Nornolatile beta
Radrin-226
Radium-226
Radium-226
Radium-226
Radium-226
Tritium
Tritium
Tritium
Tritium

Result
$<3.0 E+00$
$<3.0 E+00$
$8.9 E+00 \pm 1.9 E+00$
$1.0 E+01 \pm 1.0 E+00$
$8.9 E+00 \pm 4.0 E+00$
$2.1 E+01 \pm 4.2 E+00$
$1.6 E+00 \pm 2.4 E-01$
$<5.0 E-01$
$8.8 E-01 \pm 2.7 E-01$
$5.6 E-01 \pm 2.2 E-01$
$7.8 E-01 \pm 2.1 E-01$
$2.4 E+00 \pm 4.0 E-01$
$2.5 E+00 \pm 4.0 E-01$
$3.3 E+00 \pm 3.5 E-01$
$9.1 E+00 \pm 5.2 E-01$

Unit

$\mathrm{pCi} / \mathrm{L}$

$\mathrm{pCi} / \mathrm{L}$

$\mathrm{pCi} / \mathrm{L}$

$\mathrm{pCi} / \mathrm{L}$

$\mathrm{pCi} / \mathrm{L}$

$\mathrm{pCi} / \mathrm{L}$

$\mathrm{pCi} / \mathrm{L}$

$\mathrm{pCi/L}$

pCi/L

$\mathrm{pCi} / \mathrm{L}$

$\mathrm{pCi} / \mathrm{L}$

$\mathrm{pCi} / \mathrm{mL}$.

$\mathrm{pCi} / \mathrm{mL}$

$\mathrm{pCi} / \mathrm{mL}$

$\mathrm{pCi} / \mathrm{mL}$

$\begin{array}{ll}\text { Flag } & \text { Lab } \\ 0 & \text { CN } \\ 0 & \text { CN } \\ 0 & \text { GP } \\ 0 & \text { GP } \\ 0 & \text { CN } \\ 0 & \text { CN } \\ 0 & \text { GP } \\ 0 & \text { GP } \\ 0 & \text { CN } \\ 0 & \text { CN } \\ 0 & \text { CN } \\ 0 & \text { GP } \\ 0 & \text { GP } \\ 0 & \text { CN } \\ 0 & \text { CN }\end{array}$

Lab
CN
CN
GP
GP
CN
CN
GP
GP
CN
CN
CN
GP
GP
CN
CN

\section{WELL LFW 34}

$\begin{array}{llllll}\text { SRS Coord. } & \text { Lat/Longitude } & \text { Screen Zone Elevation } & \text { Top of Casing } & \text { Casing } & \text { Formation } \\ \text { N85409.5 } & 33.288114^{\circ} \mathrm{N} & 164.7-143.7 \mathrm{ft} \mathrm{msl} & 201.0 \mathrm{ft} \mathrm{msl} & 4^{\text {" PVC }} \\ \text { E45016.9 } & 81.714171^{\circ} \mathrm{W} & & & \end{array}$

MEASUREMENTS CONDUCTED IN THE FIELD

Sample date: 06/02/92

Depth to water: $40.89 \mathrm{ft}(12.46 \mathrm{~m})$ below TOC Water elevation: $160.11 \mathrm{ft}(48.80 \mathrm{~m}) \mathrm{msl}$

Sp. conductance: $21 \mu \mathrm{S} / \mathrm{cm}$

Water evacuated before sampling: $43 \mathrm{gal}$

\section{LABORATORY ANALYSES}

H $\underline{\text { Analyte }}$

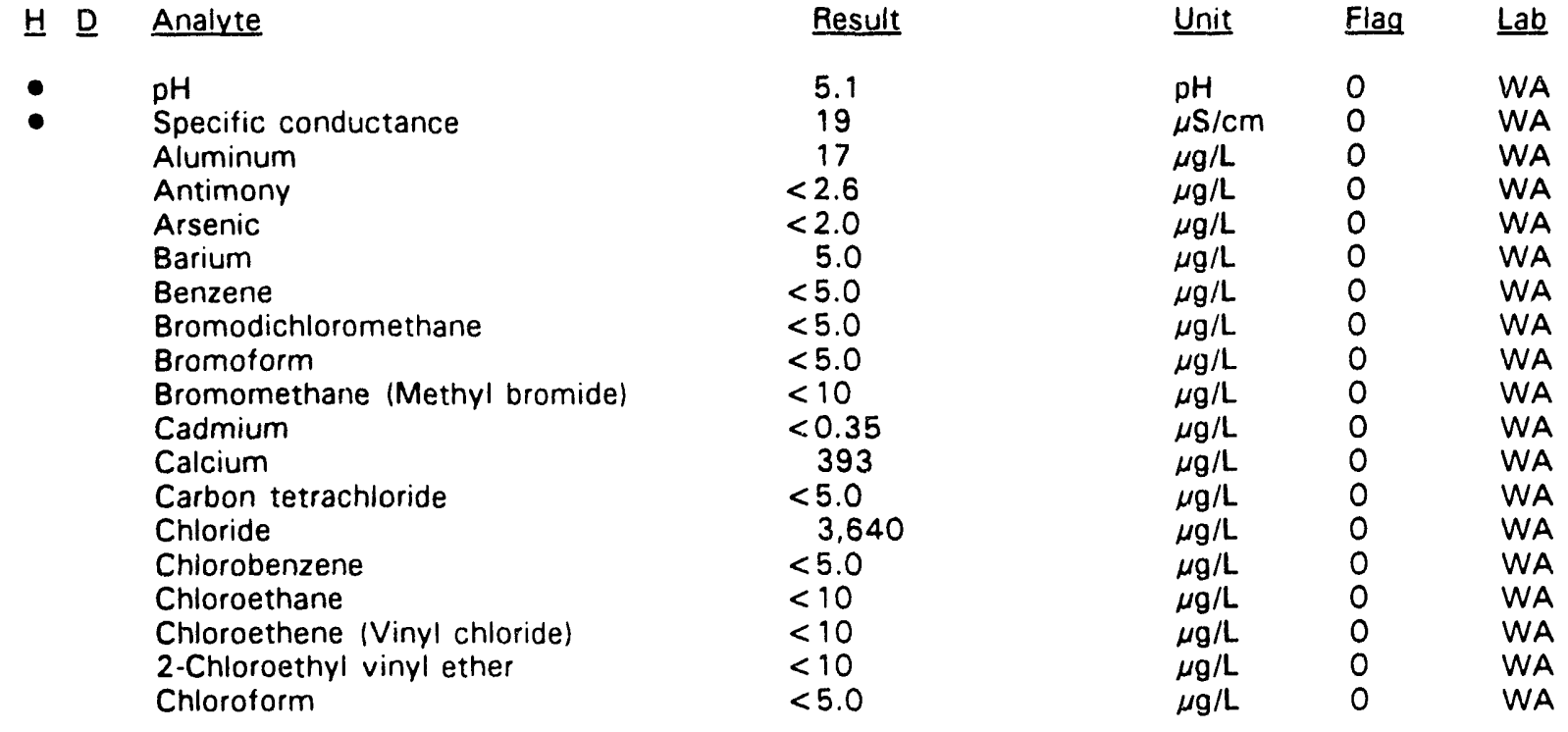

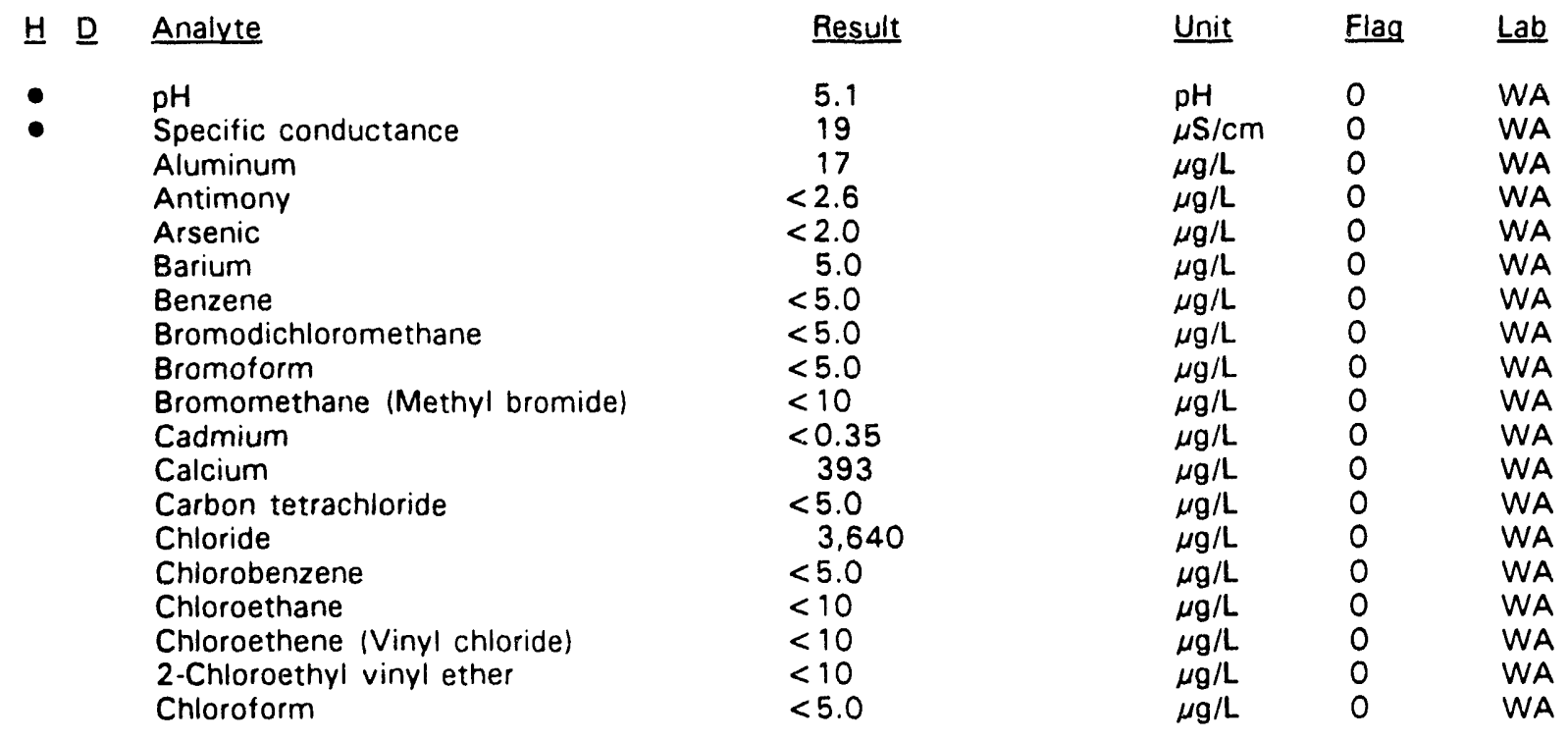

Time: 9:45

$\mathrm{pH}: 4.2$

Alkalinity: $0 \mathrm{mg} / \mathrm{L}$

Water temperature: $18.2^{\circ} \mathrm{C}$

$\overline{0}=$ exceeded holding time.

- exceeded primary drinking water standard. 
WELL LFW 34 collected on 06/02/92, laboratory analyses (cont.)

\begin{tabular}{|c|c|c|c|c|c|}
\hline$\underline{H} \underline{D}$ & Analyte & Result & $\underline{\text { Unit }}$ & Flag & $\underline{\text { L.ab }}$ \\
\hline & Chloromethane (Methyl chloride) & $<10$ & $\mu g / L$ & 0 & WA \\
\hline & Chromium & $<1.1$ & $\mu \mathrm{g} / \mathrm{L}$ & 0 & WA \\
\hline & Copper & 3.7 & $\mu g / L$ & 0 & WA \\
\hline & Dibromochloromethane & $<5.0$ & $\mu g / L$ & 0 & WA \\
\hline & 1,1-Dichloroethane & $<5.0$ & $\mu \mathrm{g} / \mathrm{L}$ & 0 & WA \\
\hline & 1,2-Dichloroethane & $<5.0$ & $\mu \mathrm{g} / \mathrm{L}$ & 0 & WA \\
\hline & cis-1,2-Dichloroethene & $<5.0$ & $\mu g / L$ & 0 & WA \\
\hline & 1,1-Dichloroethylene & $<5.0$ & $\mu g / L$ & 0 & WA \\
\hline & Dichloromethane (Methylene chloride) & 3.3 & $\mu \mathrm{g} / \mathrm{L}$ & 0 & WA \\
\hline & 2,4-Dichlorophenoxyacetic acid & $<1.1$ & $\mu \mathrm{g} / \mathrm{L}$ & 0 & WA \\
\hline & 1,2-Dichloropropane & $<5.0$ & $\mu g / L$ & 0 & WA \\
\hline & cis-1,3-Dichloropropene & $<5.0$ & $\mu g / L$ & 0 & WA \\
\hline & trans-1,3-Dichloropropene & $<5.0$ & $\mu g / L$ & 0 & WA \\
\hline & Endrin & $<0.11$ & $\mu g / L$ & 0 & WA \\
\hline & Ethylbenzene & $<5.0$ & $\mu \mathrm{g} / \mathrm{L}$ & 0 & WA \\
\hline & Fluoride & $<100$ & $\mu g / L$ & 0 & WA \\
\hline & Lead & $<2.0$ & $\mu g / L$ & 0 & WA \\
\hline & Lindane & $<0.054$ & $\mu g / L$ & 0 & WA \\
\hline & Magnesium & 400 & $\mu g / L$ & 0 & WA \\
\hline & Manganese & 2.0 & $\mu g / L$ & 0 & WA \\
\hline & Mercury & $<0.20$ & $\mu g / L$ & 0 & WA \\
\hline & Methoxychlor & $<0.54$ & $\mu g / L$ & 0 & WA \\
\hline & Nickel & $<3.1$ & $\mu \mathrm{g} / \mathrm{L}$ & 0 & WA \\
\hline & Nitrate as nitrogen & 407 & $\mu \mathrm{g} / \mathrm{L}$ & 0 & WA \\
\hline & Potassium & 600 & $\mu g / L$ & 0 & WA \\
\hline & Selenium & $<2.0$ & $\mu g / L$ & 0 & WA \\
\hline & Silica & 5.860 & $\mu g / L$ & 0 & WA \\
\hline & Silver & 0.70 & $\mu g / L$ & 0 & WA \\
\hline & Sodium & 1,490 & $\mu g / L$ & 0 & WA \\
\hline & Sulfate & 534 & $\mu g / L$ & 0 & WA \\
\hline & $1,1,2,2$-Tetrachloroethane & $<5.0$ & $\mu g / L$ & 0 & WA \\
\hline & Tetrachloroethylene & $<5.0$ & $\mu g / L$ & 0 & WA \\
\hline & Toluene & $<5.0$ & $\mu \mathrm{g} / \mathrm{L}$ & 0 & WA \\
\hline & Total organic carbon & $<500$ & $\mu g / L$ & 0 & WA \\
\hline & Total organic halogens & 21 & $\mu \mathrm{g} / \mathrm{L}$ & 0 & WA \\
\hline & Toxaphene & $<1.1$ & $\mu \mathrm{g} / \mathrm{L}$ & 0 & WA \\
\hline & 2,4,5-TP (Silvex) & $<0.54$ & $\mu \mathrm{g} / \mathrm{L}$ & 0 & WA \\
\hline & $1,1,1$-Trichloroethane & $<5.0$ & $\mu \mathrm{g} / \mathrm{L}$ & 0 & WA \\
\hline & 1,1,2-Trichloroethane & $<5.0$ & $\mu \mathrm{g} / \mathrm{L}$ & 0 & WA \\
\hline & Trichloroethylene & $<5.0$ & $\mu \mathrm{g} / \mathrm{L}$ & 0 & WA \\
\hline & Trichlorofluoromethane & $<5.0$ & $\mu \mathrm{g} / \mathrm{L}$ & 0 & WA \\
\hline & Vanadium & $<0.88$ & $\mu \mathrm{g} / \mathrm{L}$ & 0 & WA \\
\hline & Gross alpha & $<3.0 E+00$ & $\mathrm{pCi} / \mathrm{L}$ & 0 & $\mathrm{CN}$ \\
\hline & Nonvolatile beta & $<5.0 \mathrm{E}+00$ & $\mathrm{pCi} / \mathrm{L}$ & 0 & CN \\
\hline & Radium-226 & $\begin{array}{l}9.4 \mathrm{E}-01 \pm 2.3 \mathrm{E}-01 \\
2.3 \mathrm{E}+00 \pm 4.2 \mathrm{E}-01\end{array}$ & $\begin{array}{l}\mathrm{pCi} / \mathrm{L} \\
\mathrm{pCi} / \mathrm{mL}\end{array}$ & $\begin{array}{l}0 \\
0\end{array}$ & $\begin{array}{l}C N \\
C N\end{array}$ \\
\hline & 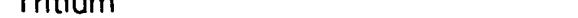 & $2.3 E+U U \pm 4 . \angle E-U 1$ & pli/mL & & CN \\
\hline
\end{tabular}

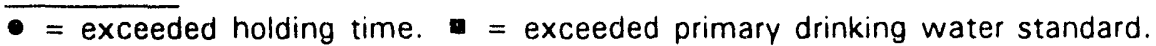


WELL LFW 35

$\begin{array}{llllll}\text { SRS Coord. } & \text { Lat/Longitude } & \text { Screen Zone Elevation } & \text { Top of Casing } & \text { Casing } & \text { Formation } \\ \text { N85237.4 } & 33.288325^{\circ} \mathrm{N} & 164.4-143.4 \mathrm{ft} \mathrm{msl} & 183.7 \mathrm{ft} \mathrm{msl} & 4^{\prime \prime} \mathrm{PVC} & \\ \text { E45378.8 } & 81.712883^{\circ} \mathrm{W} & & & \end{array}$

\section{MEASUREMENTS CONDUC TED IN THE FIELD}

Sample date: 05/29/92

Depth to water: $24.82 \mathrm{ft}(7.57 \mathrm{~m})$ below TOC

Water elevation: $158.88 \mathrm{ft}(48.43 \mathrm{~m}) \mathrm{ms}$

Sp. conductance: $25 \mu \mathrm{S} / \mathrm{cm}$

Water evacuated before sampling: $51 \mathrm{gal}$

Time: $14: 50$

$\mathrm{pH}: 4.4$

Alkalinity: $1 \mathrm{mg} / \mathrm{L}$

Water temperature: $19.7^{\circ} \mathrm{C}$

\section{LABORATORY ANALYSES}

H $\underline{\text { Analyte }}$

- $\mathrm{pH}$

- Specific conductance

Aluminum

- Antimony

Arsenic

Barium

Benzene

Bromodichloromethane

Bromoform

Bromomethane (Methyl bromide)

Cadmium

Calcium

Carbon tetrachloride

Chloride

Chlorobenzene

Chloroethane

Chloroethene (Vinyl chloride)

2-Chloroethyl vinyl ether

Chloroform

Chloromethane (Methyl chloride)

Chromium

Copper

Dibromochloromethane

1,1-Dichloroethane

1.2-Dichloroethane

cis-1,2-Dichloroethene

1,1-Dichloroethylene

Dichloromethane (Methylene chloride)

2,4-Dichlorophenoxyacetic acid

1,2-Dichloropropane

cis-1,3-Dichloropropene

trans-1,3-Dichloropropene

Endrin

Ethylbenzene

Fluoride

Lead

Lindane

Magnesium

Manganese
Result

\section{2}

23

21

10

$<2.0$

6.9

$<5.0$

$<5.0$

$<5.0$

$<10$

2.3

386

$<5.0$

3,060

$<5.0$

$<10$

$<10$

$<10$

$<5.0$

$<10$

$<1.1$

3.3

$<5.0$

$<5.0$

$<5.0$

$<5.0$

$<5.0$

$<5.0$

$<1.1$

$<5.0$

$<5.0$

$<5.0$

$<0.11$

$<5.0$

$<100$

$<2.0$

$<0.056$

694

4.0
Unit $\quad \underline{\text { Flag }} \underline{\text { Lab }}$

$\mathrm{pH} \quad 0 \quad$ WA

$\mu \mathrm{S} / \mathrm{cm} \quad 0 \quad$ WA

$\mu g / L \quad$ WA

$\mu g / L \quad 2 \quad$ WA

$\mu g / L \quad 0 \quad$ WA

$\mu g / L \quad 0 \quad$ WA

$\mu g / L \quad$ WA

$\mu g / L \quad 0 \quad W A$

$\mu \mathrm{g} / \mathrm{L} \quad 0 \quad$ WA

$\mu g / L \quad 0 \quad$ WA

$\mu g / L \quad 0 \quad$ WA

$\mu g / L \quad 0 \quad$ WA

$\mu g / L \quad$ WA

$\mu g / L . \quad 0 \quad$ WA

$\mu g / L \quad 0 \quad$ WA

$\mu g / L \quad 0 \quad$ WA

$\mu \mathrm{g} / \mathrm{L} \quad 0 \quad$ WA

$\mu g / L \quad 0 \quad$ WA

$\mu g / L \quad 0$

$\mu g / L \quad 0 \quad W A$

$\mu \mathrm{g} / \mathrm{L} \quad 0$

$\mu g / L \quad 0 \quad W A$

$\mu g / L \quad 0$

$\mu g / L \quad 0 \quad W A$

$\mu g / L \quad 0$

$\mu g / L \quad 0 \quad W A$

$\mu g / L \quad 0 \quad W A$

$\mu \mathrm{g} / \mathrm{L} \quad 0 \quad$ WA

$\mu g / L \quad 0 \quad W A$

$\mu g / L \quad 0 \quad W A$

$\mu g / L \quad 0 \quad W A$

$\mu g / L \quad 0 \quad W A$

$\mu g / L \quad 0 \quad$ WA

$\mu g / L \quad 0 \quad W A$

$\mu g i L \quad$ WA

$\mu \mathrm{g} / \mathrm{L} \quad 0 \quad$ WA

$\mu \mathrm{g} / \mathrm{L} \quad 0 \quad$ WA

$\mu g / L \quad 0 \quad W A$

$\mu g / L \quad 0 \quad$ WA

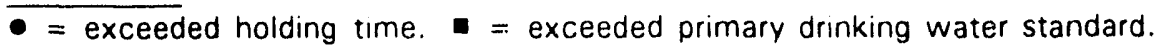


WELL. LFW 35 collected on 05/29/92, laboratory analyses (cont.)

H. $\quad$ Analyte
Mercury
Methoxychlor
Nickel
Nitrate as nitrogen
Potassium
Selenium
Silica
Silver
Sodium
Sulfate
1,1,2,2-Tetrachloroethane
Tetrachloroethylene
Toluene
Total organic carbon
Total organic halogens
Toxaphene
2,4,5-TP (Silvex)
1,1,1-Trichloroethane
1,1,2-Trichloroethane
Trichloroethylene
Trichlorofluoromethane
Vanadium
Gross alpha
Nonvolatile beta
Radium-226
Tritium

\begin{tabular}{llll}
\multicolumn{1}{r}{ Result } & Unit & Flag & Lab \\
$<0.20$ & $\mu \mathrm{g} / \mathrm{L}$ & 0 & WA \\
$<0.56$ & $\mu \mathrm{g} / \mathrm{L}$ & 0 & WA \\
$<3.1$ & $\mu \mathrm{g} / \mathrm{L}$ & 0 & WA \\
1.120 & $\mu \mathrm{g} / \mathrm{L}$ & 0 & WA \\
549 & $\mu \mathrm{g} / \mathrm{L}$ & 0 & WA \\
$<2.0$ & $\mu \mathrm{g} / \mathrm{L}$ & 0 & WA \\
5.800 & $\mu \mathrm{g} / \mathrm{L}$ & 0 & WA \\
$<0.70$ & $\mu \mathrm{g} / \mathrm{L}$ & 0 & WA \\
2.350 & $\mu \mathrm{g} / \mathrm{L}$ & 0 & WA \\
251 & $\mu \mathrm{g} / \mathrm{L}$ & 0 & WA \\
$<5.0$ & $\mu \mathrm{g} / \mathrm{L}$ & 0 & WA \\
$<5.0$ & $\mu \mathrm{g} / \mathrm{L}$ & 0 & WA \\
$<5.0$ & $\mu \mathrm{g} / \mathrm{L}$ & 0 & WA \\
1,960 & $\mu \mathrm{g} / \mathrm{L}$ & 0 & WA \\
$<20$ & $\mu \mathrm{g} / \mathrm{L}$ & 0 & WA \\
$<1.1$ & $\mu \mathrm{g} / \mathrm{L}$ & 0 & WA \\
$<0.56$ & $\mu \mathrm{g} / \mathrm{L}$ & 0 & WA \\
$<5.0$ & $\mu \mathrm{g} / \mathrm{L}$ & 0 & WA \\
$<5.0$ & $\mu \mathrm{g} / \mathrm{L}$ & 0 & WA \\
$<5.0$ & $\mu \mathrm{g} / \mathrm{L}$ & 0 & WA \\
$<5.0$ & $\mu \mathrm{g} / \mathrm{L}$ & 0 & WA \\
$<0.88$ & $\mu \mathrm{g} / \mathrm{L}$ & 0 & WA \\
$<3.0 \mathrm{E}+00$ & $\mathrm{pCi} / \mathrm{L}$ & 0 & CN \\
$<5.0 \mathrm{E}+00$ & $\mathrm{pCi} / \mathrm{L}$ & 0 & CN \\
$9.7 \mathrm{E}-01 \pm 2.5 \mathrm{E}-01$ & $\mathrm{DCi} / \mathrm{L}$ & 0 & CN \\
$<2.0 \mathrm{E}+00$ & $\mathrm{DCi} / \mathrm{mL}$ & 0 & CN
\end{tabular}

\section{WELL LFW 36}

$\begin{array}{llllll}\text { SRS Coord. } & \text { Lat/Longitude } & \text { Screen Zone Elevation } & \text { Top of Casing } & \text { Casing } & \text { Formation } \\ \text { N83535.5 } & 33.284894 \mathrm{o} & 151.3-130.3 \mathrm{ft} \mathrm{msl} & 170.4 \mathrm{ft} \mathrm{msl} & \text { 4" PVC } \\ \text { E45582.3 } & 81.709040^{\circ} \mathrm{W} & & & \end{array}$

\section{MEASUREMENTS CONDUCTED IN THE FIELD}

Sample date: 06/04/92

Depth to water: $24.75 \mathrm{ft}(7.54 \mathrm{~m})$ below TOC

Water elevation: $145.65 \mathrm{ft}(44.39 \mathrm{~m}) \mathrm{msl}$

Sp. conductance: $323 \mu \mathrm{S} / \mathrm{cm}$

Water evacuated before sampling: $50 \mathrm{gal}$

\section{LABORATORY ANALYSES}

$\begin{array}{ll}\text { H } D \quad & \text { Analyte } \\ \text { - } & \text { pH } \\ & \text { Specific conductance } \\ & \text { Aluminum } \\ & \text { Antimony } \\ & \text { Arsenic } \\ & \text { Barium } \\ \text { - Benzene } \\ \text { Bromodichloromethane }\end{array}$

Time: $12: 45$

pH: 6.2

Alkalinity: $94 \mathrm{mg} / \mathrm{L}$

Water temperature: $19.8^{\circ} \mathrm{C}$

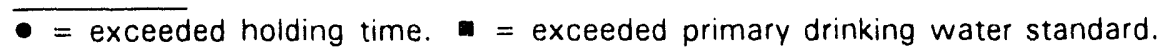

$\begin{array}{llll}\text { Result } & \text { Unit } & \text { Flag } & \text { Lab } \\ 6.2 & & & \text { WH } \\ 264 & \mu \mathrm{S} / \mathrm{cm} & 0 & 1 \\ <15 & \mu \mathrm{g} / \mathrm{L} & 0 & \text { WA } \\ <2.6 & \mu \mathrm{g} / \mathrm{L} & 0 & \text { WA } \\ <2.0 & \mu \mathrm{g} / \mathrm{L} & 0 & \text { WA } \\ <4.0 & \mu \mathrm{g} / \mathrm{L} & 0 & \text { WA } \\ 5.8 & \mu \mathrm{g} / \mathrm{L} & 2 & \text { WA } \\ <5.0 & \mu \mathrm{g} / \mathrm{L} & 0 & \text { WA }\end{array}$


WELL LFW 36 collected on 06/04/92, laboratory analyses (cont.)

H $\underline{\text { Analyte }}$

Bromoform

Bromomethane (Methyl bromide)

Cadmium

Calcium

Carbon tetrachloride

Chloride

Chloride

Chlorobenzene

Chloroethane

Chloroethene (Vinyl chloride)

2-Chloroethyl vinyl ether

Chloroform

Chloromethane (Methyl chloride)

Chromium

Copper

Dibromochloromettiane

1,1-Dichloroethane

1.2-Dichloroethane

cis-1,2-Dichloroethene

1,1-Dichloroethylene

Dichloromethane (Methylene chloride)

2,4-Dichlorophenoxyacetic acid

1,2-Dichloropropane

cis-1,3-Dichloropropene

trans-1,3-Dichloropropene

Endrin

Ethylbenzene

Fluoride

Fluoride

Lead

Lindane

Magnesium

Manganese

Mercury

Methoxychlor

Nickel

Nitrate as nitrogen

Potassium

Selenium

Silica

Silver

Sodium

Sulfate

1,1,2,2-Tetrachloroethane

Tetrachloroethylene

Toluene

Total organic carbon

Total organic halogens

Toxaphene

2,4,5-TP (Silvex)

1,1,1-Trichloroethane

1,1,2-Trichloroethane

Trichloroethylene

Trichlorofluoromethane

Vanadium

Gross alpha

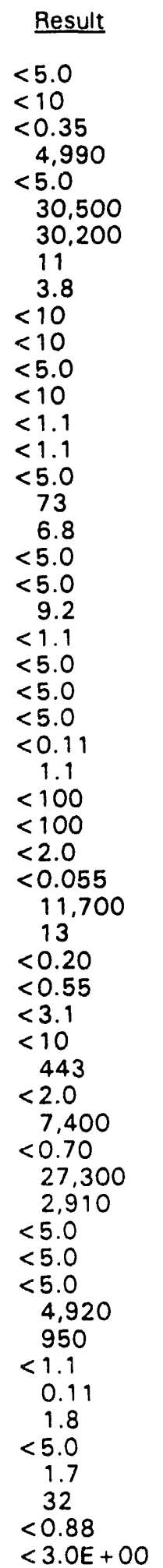

\begin{tabular}{|c|c|c|}
\hline Unit & Flag & $\underline{L a b}$ \\
\hline$\mu g / L$ & 0 & WA \\
\hline$\mu g / L$ & 0 & WA \\
\hline$\mu \mathrm{g} / \mathrm{L}$ & 0 & WA \\
\hline$\mu \mathrm{g} / \mathrm{L}$ & 0 & WA \\
\hline$\mu \mathrm{g} / \mathrm{L}$ & 0 & WA \\
\hline$\mu \mathrm{g} / \mathrm{L}$ & 0 & WA \\
\hline$\mu \mathrm{g} / \mathrm{L}$ & 0 & WA \\
\hline$\mu g / L$ & 2 & WA \\
\hline$\mu \mathrm{g} / \mathrm{L}$ & 0 & WA \\
\hline$\mu \mathrm{g} / \mathrm{L}$ & 0 & WA \\
\hline$\mu g / L$ & 0 & WA \\
\hline$\mu g / L$ & 0 & WA \\
\hline$\mu \mathrm{g} / \mathrm{L}$ & 0 & WA \\
\hline$\mu g / L$ & 0 & WA \\
\hline$\mu g / L$ & 0 & WA \\
\hline$\mu g / L$ & 0 & WA \\
\hline$\mu g / L$ & 2 & WA \\
\hline$\mu \mathrm{g} / \mathrm{L}$ & 2 & WA \\
\hline$\mu \mathrm{g} / \mathrm{L}$ & 0 & WA \\
\hline$\mu g / L$ & 0 & WA \\
\hline$\mu g / L$ & 0 & WA \\
\hline$\mu g / L$ & 0 & WA \\
\hline$\mu g / L$ & 0 & WA \\
\hline$\mu g / L$ & 0 & WA \\
\hline$\mu g / L$ & 0 & WA \\
\hline$\mu g / L$ & 0 & WA \\
\hline$\mu \mathrm{g} / \mathrm{L}$ & 0 & WA \\
\hline$\mu g / L$ & 0 & WA \\
\hline$\mu g / L$ & 0 & WA \\
\hline$\mu g / L$ & 0 & WA \\
\hline$\mu g / L$ & 0 & WA \\
\hline$\mu g / L$ & 0 & WA \\
\hline$\mu \mathrm{g} / \mathrm{L}$ & 0 & W \\
\hline$\mu \mathrm{g} / \mathrm{L}$ & 0 & W \\
\hline$\mu g / L$ & 0 & WA \\
\hline$\mu g / L$ & 0 & WA \\
\hline$\mu g / L$ & 0 & WA \\
\hline$\mu \mathrm{g} / \mathrm{L}$ & 0 & WA \\
\hline$\mu \mathrm{g} / \mathrm{L}$ & 0 & WA \\
\hline$\mu \mathrm{g} / \mathrm{L}$ & 0 & WA \\
\hline$\mu g / L$ & 0 & WA \\
\hline$\mu g / L$ & 0 & WA \\
\hline$\mu \mathrm{g} / \mathrm{L}$ & 0 & WA \\
\hline$\mu g / L$ & 0 & WA \\
\hline$\mu g / L$ & 0 & WA \\
\hline$\mu g / L$ & 0 & WA \\
\hline$\mu g / L$ & 0 & WA \\
\hline$\mu g / L$ & 2 & WA \\
\hline$\mu g / L$ & 0 & W \\
\hline$\mu g / L$ & 0 & W \\
\hline$\mu \mathrm{g} / \mathrm{L}$ & 0 & WA \\
\hline$\mu g / L$ & 0 & WA \\
\hline$\mu g / L$ & 0 & WA \\
\hline$\mu \mathrm{g} / \mathrm{L}$ & 2 & WA \\
\hline$\mu \mathrm{g} / \mathrm{L}$ & 0 & W \\
\hline $\mathrm{pCi} / \mathrm{L}$ & 0 & $\mathrm{Cl}$ \\
\hline
\end{tabular}

$\overline{-=}$ exceeded holding time.

- =xceeded primary drinking water standard.

Sanitary Landfill 
WELL LFW 36 collected on 06/04/92, laboratory analyses (cont.)

$\begin{array}{lllll}\text { H } \underline{D} \text { Analyte } & \underline{\text { Result }} & \underline{\text { Urii }} & \underline{\text { Flag }} & \underline{\text { Lab }} \\ \text { Nonvolatile beta } & <5.0 \mathrm{E}+00 & \mathrm{pCi} / \mathrm{L} & 0 & \mathrm{CN} \\ \text { Radium-226 } & <5.0 \mathrm{E}-01 & \mathrm{pCi} / \mathrm{L} & 0 & \mathrm{CN} \\ \text { Tritium } & 7.8 \mathrm{E}+00 \pm 4.9 \mathrm{E}-01 \mathrm{pCi/mL} & 0 & \mathrm{CN}\end{array}$

\section{WELL LFW 37}

\begin{tabular}{|c|c|c|c|c|}
\hline SRS Coord. & Lat/Longitude & Screen Zone Elevation & Top of Casing & Casing \\
\hline $\begin{array}{l}\text { N83113.2 } \\
\text { E45667.7 }\end{array}$ & $\begin{array}{l}33.284100^{\circ} \mathrm{N} \\
81.707994^{\circ} \mathrm{W}\end{array}$ & $150.8-129.8 \mathrm{ft} \mathrm{msl}$ & 169.9 & 4 \\
\hline
\end{tabular}

\section{MEASUREMENTS CONDUCTED IN THE FIELD}

Sample date: 06/03/92

Depth to water: $27.29 \mathrm{ft}(8.32 \mathrm{~m})$ below TOC

Water elevation: $142.61 \mathrm{ft}(43.47 \mathrm{~m}) \mathrm{ms}$ l

Sp. conductance: $184 \mu \mathrm{S} / \mathrm{cm}$

Water evacuated before sampling: $34 \mathrm{gal}$

\section{LABORATORY ANALYSES}

Time: 1 s:20

pH: 4.7

Alkalinity: $16 \mathrm{mg} / \mathrm{L}$

Water temperature: $19.1^{\circ} \mathrm{C}$

\begin{tabular}{|c|c|c|c|c|c|}
\hline$\underline{D}$ & Analyte & Result & Unit & Flag & $\underline{L a b}$ \\
\hline & $\mathrm{pH}$ & 5.8 & $\mathrm{pH}$ & 0 & WA \\
\hline & $\mathrm{pH}$ & 5.8 & $\mathrm{pH}$ & 0 & WA \\
\hline & Specific conductance & 155 & $\mu \mathrm{S} / \mathrm{cm}$ & 0 & WA \\
\hline & Specific conductance & 157 & $\mu \mathrm{S} / \mathrm{cm}$ & 0 & WA \\
\hline & Aluminum & 16 & $\mu \mathrm{g} / \mathrm{L}$ & 0 & WA \\
\hline a & Antimony & 9.9 & $\mu g / L$ & 2 & WA \\
\hline & Arsenic & $<2.0$ & $\mu g / L$ & 0 & WA \\
\hline$=$ & $\begin{array}{l}\text { Barium } \\
\text { Benzene }\end{array}$ & $\begin{array}{l}18 \\
5.6\end{array}$ & $\mu g / L$ & $\begin{array}{l}0 \\
2\end{array}$ & $\begin{array}{l}\text { WA } \\
\text { WA }\end{array}$ \\
\hline 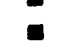 & $\begin{array}{l}\text { Benzene } \\
\text { Benzene }\end{array}$ & $\begin{array}{l}5.6 \\
5.2\end{array}$ & $\mu g / L$ & 2 & WA \\
\hline & Bromodichloromethane & $<5.0$ & $\mu g / L$ & 0 & WA \\
\hline & Bromodichloromethane & $<5.0$ & $\mu g / L$ & 0 & WA \\
\hline & Bromodichloromethane & $<5.0$ & $\mu g / L$ & 0 & WA \\
\hline & Bromoform & $<5.0$ & $\mu \mathrm{g} / \mathrm{L}$ & 0 & WA \\
\hline & Bromoform & $<5.0$ & $\mu \mathrm{g} / \mathrm{L}$ & 0 & WA \\
\hline & Bromoform & $<5.0$ & $\mu g / L$ & 0 & WA \\
\hline & Bromomethane (Methyl bromide) & $<10$ & $\mu \mathrm{g} / \mathrm{L}$ & 0 & WA \\
\hline & Bromomethane (Methyl bromide) & $<10$ & $\mu g / L$ & 0 & WA \\
\hline & Bromomethane (Methyi bromide) & $<10$ & $\mu g / L$ & 0 & WA \\
\hline & Cadmium & 0.42 & $\mu g / L$ & 0 & WA \\
\hline & Calcium & 2,870 & $\mu g / L$ & 0 & WA \\
\hline & Carbon tetrachloride & $<5.0$ & $\mu g / L$ & 0 & WA \\
\hline & Carbon tetrachloride & $<5.0$ & $\mu g / L$ & 0 & WA \\
\hline & Carbon tetrachloride & $<5.0$ & $\mu g / L$ & 0 & WA \\
\hline & Chloride & 29,400 & $\mu g / L$ & 0 & WA \\
\hline & Chlorobenzene & $<5.0$ & $\mu \mathrm{g} / \mathrm{L}$ & 0 & WA \\
\hline & Chlorobenzene & $<5.0$ & $\mu g / L$ & 0 & WA \\
\hline & Chloroethane & $<10$ & $\mu g / L$ & 0 & WA \\
\hline & Chloroethane & $<10$ & $\mu \mathrm{g} / \mathrm{L}$ & 0 & WA \\
\hline & Chloroethane & $<10$ & $\mu \mathrm{g} / \mathrm{L}$ & 0 & WA \\
\hline & Chloroethene (Vinyl chloride) & $<10$ & $\mu g / L$ & 0 & WA \\
\hline
\end{tabular}

- = exceeded holding time. = exceeded primary drinking water standard. 
WELL LFW 37 collected on 06/03/92, laboratory analyses (cont.)

H D Analyte

Chloroethene (Vinyl chloride)

Chloroethene (Vinyl chloride)

2-Chloroethyl vinyl ether

2-Chloroethyl vinyl ether

2-Chloroethyl vinyl ether

Chloroform

Chloroform

Chloroform

Chloromethane (Methyl chloride)

Chloromethane (Methyl chloride)

Chloromethane (Methyl chloride)

Chromium

Copper

Dibromochloromethane

Dibromochloromethane

Dibromochloromethane

1,1-Dichloroethane

1,1-Dichloroethane

1,1-Dichloroethane

- 1,2-Dichloroethane

- 1,2-Dichloroethane

- 1,2-Dichloroethane cis-1,2-Dichloroethene cis-1,2-Dichloroethene cis-1,2-Dichloroethene 1,1-Dichloroethylene 1,1-Dichloroethylene

Dichloromethane (Methylene chloride)

Dichloromethane (Methylene chloride)

Dichloromethane (Methylene chloride)

2,4-Dichlorophenoxyacetic acid

1,2-Dichloropropane

1,2-Dichloropropane

1,2-Dichloropropane

cis-1,3-Dichloropropene

cis-1,3-Dichloropropene

cis-1,3-Dichloropropene

trans-1,3-Dichloropropene

trans-1,3-Dichloropropene

trans-1,3-Dichloropropene

Endrin

Ethylbenzene

Ethylbenzene

Ethylbenzene

Fluoride

Lead

Lindane

Magnesium

Manganese

Mercury

Mercury

Methoxychlor

Nickel

Nitrate as nitrogen

Potassium

Selenium

\section{Result}

$<10$

$<10$

$<10$

$<10$

$<10$

$<5.0$

$<5.0$

$<5.0$

$<10$

$<10$

$<10$

$<1.1$

1.4

$<5.0$

$<5.0$

$<5.0$

98

96

92

7.1

7.8

6.6

$<5.0$

$<5.0$

$<5.0$

1.5

1.6

32

31

27

$<1.1$

$<5.0$

$<5.0$

$<5.0$

$<5.0$

$<5.0$

$<5.0$

$<5.0$

$<5.0$

$<5.0$

$<0.11$

$<5.0$

$<5.0$

$<5.0$

$<100$

$<2.0$

$<0.054$

5,170

0.85

$<0.20$

$<0.20$

$<0.54$

$<3.1$

222

403

$<2.0$

\begin{tabular}{lll} 
Unit & Flag & Lab \\
\hline$\mu g / L$ & 0 & WA \\
$\mu g / L$ & 0 & WA \\
$\mu g / L$ & 0 & WA \\
$\mu g / L$ & 0 & WA \\
$\mu g / L$ & 0 & WA \\
$\mu g / L$ & 0 & WA \\
$\mu g / L$ & 0 & WA \\
$\mu g / L$ & 0 & WA \\
$\mu g / L$ & 0 & WA \\
$\mu g / L$ & 0 & WA \\
$\mu g / L$ & 0 & WA \\
$\mu g / L$ & 0 & WA \\
$\mu g / L$ & 0 & WA \\
$\mu g / L$ & 0 & WA \\
$\mu g / L$ & 0 & WA \\
$\mu g / L$ & 0 & WA \\
$\mu g / L$ & 2 & WA \\
$\mu g / L$ & 2 & WA \\
$\mu g / L$ & 2 & WA \\
$\mu g / L$ & 2 & WA \\
$\mu g / L$ & 2 & WA \\
$\mu g / L$ & 2 & WA \\
$\mu g / L$ & 0 & WA \\
$\mu g / L$ & 0 & WA \\
$\mu g / L$ & 0 & WA \\
$\mu g / L$ & 0 & WA \\
$\mu g / L$ & 0 & WA \\
$\mu g / L$ & 0 & WA \\
$\mu g / L$ & 0 & WA \\
$\mu g / L$ & 0 & WA \\
$\mu g / L$ & 0 & WA \\
$\mu g / L$ & 0 & WA \\
$\mu g / L$ & 0 & WA \\
$\mu g / L$ & 0 & WA \\
$\mu g / L$ & 0 & WA \\
$\mu g / L$ & 0 & WA \\
$\mu g / L$ & 0 & WA \\
$\mu g / L$ & 0 & WA \\
$\mu g / L$ & 0 & WA \\
$\mu g / L$ & 0 & WA \\
$\mu g / L$ & 0 & WA \\
$\mu g / L$ & 0 & WA \\
$\mu g / L$ & 0 & WA \\
$\mu g / L$ & 0 & WA \\
$\mu g / L$ & 0 & WA \\
$\mu g / L$ & 0 & WA \\
$\mu g / L$ & 0 & WA \\
$\mu g / L$ & 0 & WA \\
$\mu g / L$ & 0 & WA \\
$\mu g / L$ & 0 & WA \\
$\mu g / L$ & 0 & WA \\
$\mu g / L$ & 0 & WA \\
$\mu g / L$ & 0 & WA \\
$\mu g / L$ & 0 & WA \\
$\mu g / L$ & 0 & WA \\
$\mu g / L$ & 0 & WA \\
& & \\
\hline & 0 & 0
\end{tabular}

$\overline{- \text { = exceeded holding time. }}$ = exceeded primary drinking water standard. 
WELL LFW 37 collected on 06/03/92, laboratory analyses (cont.)

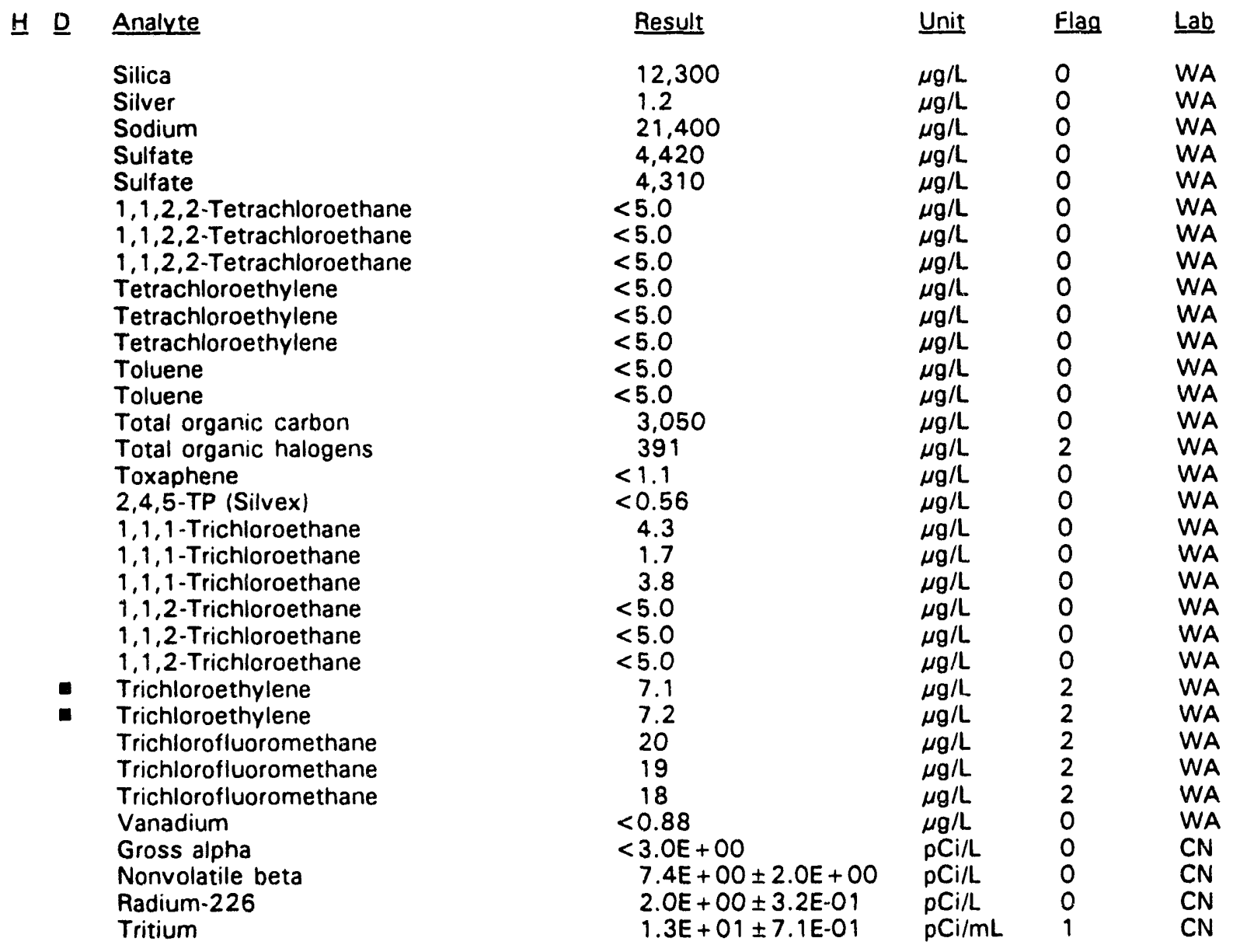

$\overline{- \text { = exceeded holding time. }}$ = exceeded primary drinking water standard. 
WELL LFW 38

$\begin{array}{llllll}\text { SRS Coord. Lat/Longitude } & \text { Screen Zone Elevation } & \text { Top of Casing } & \text { Casing } & \text { Formation } \\ \text { N83172.3 } & 33.284803^{\circ} \mathrm{N} & 151.5-130.5 \mathrm{ft} \mathrm{msl} & 170.3 \mathrm{ft} \mathrm{msl} & 4^{\text {"PVC }} & \text { PV }\end{array}$

E46018.5 $81.707185^{\circ} \mathrm{W} \quad 151.5-130.5 \mathrm{ft} \mathrm{msl} \quad 170.3 \mathrm{ft} \mathrm{msl} \quad 4$ "PVC

\section{MEASUREMENTS CONDUCTED IN THE FIELD}

Sample date: 06/03/92

Depth to water: $27.33 \mathrm{ft}(8.33 \mathrm{~m})$ below TOC Water elevation: $142.97 \mathrm{ft}(43.58 \mathrm{~m} / \mathrm{msl}$

Sp. conductance: $61 \mu \mathrm{S} / \mathrm{cm}$

Water evacuated before sampling: $33 \mathrm{gal}$

\section{LABORATORY ANALYSES}

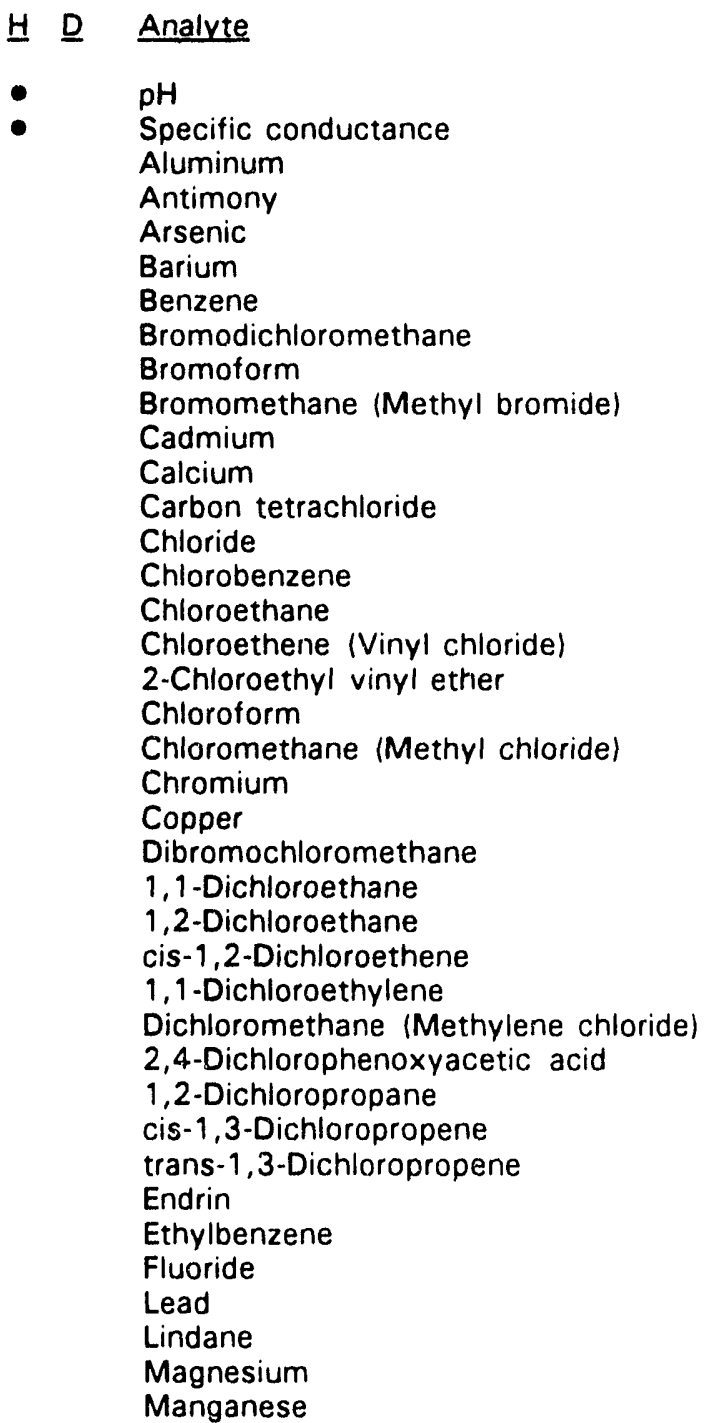

Time: 12:35

pH: 4.0

Alkalinity: $0 \mathrm{mg} / \mathrm{L}$

Water temperature: $20.3^{\circ} \mathrm{C}$

\begin{tabular}{llll} 
Result & Unit & Flag & Lab \\
\hline 5.1 & $\mu \mathrm{pH}$ & 0 & WA \\
53 & $\mu \mathrm{S} / \mathrm{cm}$ & 0 & WA \\
34 & $\mu \mathrm{g} / \mathrm{L}$ & 0 & WA \\
$<2.6$ & $\mu \mathrm{g} / \mathrm{L}$ & 0 & WA \\
$<2.0$ & $\mu \mathrm{g} / \mathrm{L}$ & 0 & WA \\
16 & $\mu \mathrm{L} / \mathrm{L}$ & 0 & WA \\
$<5.0$ & $\mu \mathrm{g} / \mathrm{L}$ & 0 & WA \\
$<5.0$ & $\mu \mathrm{g} / \mathrm{L}$ & 0 & WA \\
$<5.0$ & $\mu \mathrm{g} / \mathrm{L}$ & 0 & WA \\
$<10$ & $\mu \mathrm{g} / \mathrm{L}$ & 0 & WA \\
$<0.35$ & $\mu \mathrm{g} / \mathrm{L}$ & 0 & WA \\
772 & $\mu \mathrm{g} / \mathrm{L}$ & 0 & WA \\
$<5.0$ & $\mu \mathrm{g} / \mathrm{L}$ & 0 & WA \\
15.500 & $\mu \mathrm{g} / \mathrm{L}$ & 0 & WA \\
$<5.0$ & $\mu \mathrm{g} / \mathrm{L}$ & 0 & WA \\
$<10$ & $\mu \mathrm{g} / \mathrm{L}$ & 0 & WA \\
$<10$ & $\mu \mathrm{g} / \mathrm{L}$ & 0 & WA \\
$<10$ & $\mu \mathrm{g} / \mathrm{L}$ & 0 & WA \\
$<5.0$ & $\mu \mathrm{g} / \mathrm{L}$ & 0 & WA \\
$<10$ & $\mu \mathrm{g} / \mathrm{L}$ & 0 & WA \\
$<1.1$ & $\mu \mathrm{g} / \mathrm{L}$ & 0 & WA \\
3.2 & $\mu \mathrm{g} / \mathrm{L}$ & 0 & WA \\
$<5.0$ & $\mu \mathrm{g} / \mathrm{L}$ & 0 & WA \\
58 & $\mu \mathrm{g} / \mathrm{L}$ & 2 & WA \\
3.9 & $\mu \mathrm{g} / \mathrm{L}$ & 1 & WA \\
$<5.0$ & $\mu \mathrm{g} / \mathrm{L}$ & 0 & WA \\
2.9 & $\mu \mathrm{g} / \mathrm{L}$ & 0 & WA \\
56 & $\mu \mathrm{g} / \mathrm{L}$ & 0 & WA \\
$<1.1$ & $\mu \mathrm{g} / \mathrm{L}$ & 0 & WA \\
$<5.0$ & $\mu \mathrm{g} / \mathrm{L}$ & 0 & WA \\
$<5.0$ & $\mu \mathrm{g} / \mathrm{L}$ & 0 & WA \\
$<5.0$ & $\mu \mathrm{g} / \mathrm{L}$ & 0 & WA \\
$<0.11$ & $\mu \mathrm{g} / \mathrm{L}$ & 0 & WA \\
$<5.0$ & $\mu \mathrm{g} / \mathrm{L}$ & 0 & WA \\
$<100$ & $\mu \mathrm{g} / \mathrm{L}$ & 0 & WA \\
$<2.0$ & $\mu \mathrm{g} / \mathrm{L}$ & 0 & WA \\
$<0.056$ & $\mu \mathrm{g} / \mathrm{L}$ & 0 & WA \\
1.060 & $\mu \mathrm{g} / \mathrm{L}$ & 0 & WA \\
4.8 & $\mu \mathrm{g} / \mathrm{L}$ & 0 & WA
\end{tabular}

- = exceeded holding time. = exceeded primary drinking water standard. 
WELL LFW 38 collected on 06/03/92, laboratory analyses (cont.)

$\begin{array}{ll}\text { H. } & \text { Analyte } \\ \text { Mercury } \\ \text { Methoxychlor } \\ \text { Nickel } \\ \text { Nitrate as nitrogen } \\ \text { Potassium } \\ \text { Selenium } \\ \text { Silica } \\ \text { Silver } \\ \text { Sodium } \\ \text { Sulfate } \\ \text { 1,1,2,2-Tetrachloroethane } \\ \text { Tetrachioroethylene } \\ \text { Toluene } \\ \text { Total organic carbon } \\ \text { Total organic halogens } \\ \text { Toxaphene } \\ \text { 2,4,5-TP (Silvex) } \\ \text { 1,1,1-Trichloroethane } \\ \text { 1,1,2-Trichloroethane } \\ \text { Trichloroethylene } \\ \text { Trichlorofluoromethane } \\ \text { Vanadium } \\ \text { Gross alpha } \\ \text { Nonvolatile beta } \\ \text { Radium-226 } \\ \text { Tritium }\end{array}$

$\begin{aligned} & \text { Result } \\ &< 0.20 \\ &< 0.56 \\ &< 3.1 \\ & 153 \\ & 463 \\ &< 2.0 \\ & 11,500 \\ &< 0.70 \\ & 8.200 \\ &< 2,500 \\ &< 5.0 \\ & 1.8 \\ &< 5.0 \\ & 1,080 \\ & 254 \\ &< 1.1 \\ &< 0.56 \\ & 6.7 \\ &<5.0 \\ & 18 \\ & 17 \\ &<0.88 \\ &<3.0 \mathrm{E}+00 \\ &<5.0 \mathrm{E}+00 \\ & 6.3 \mathrm{E}-01 \pm 1.8 \mathrm{E}-01 \\ & 3.8 \mathrm{E}+01 \pm 1.1 \mathrm{E}+00\end{aligned}$

$\begin{array}{lll}\text { Unit } & \text { Flag } & \text { Lab } \\ \mu \mathrm{g} / \mathrm{L} & 0 & \text { WA } \\ \mu \mathrm{g} / \mathrm{L} & 0 & \text { WA } \\ \mu \mathrm{g} / \mathrm{L} & 0 & \text { WA } \\ \mu \mathrm{g} / \mathrm{L} & 0 & \text { WA } \\ \mu \mathrm{g} / \mathrm{L} & 0 & \text { WA } \\ \mu \mathrm{g} / \mathrm{L} & 0 & \text { WA } \\ \mu \mathrm{g} / \mathrm{L} & 0 & \text { WA } \\ \mu \mathrm{g} / \mathrm{L} & 0 & \text { WA } \\ \mu \mathrm{g} / \mathrm{L} & 0 & \text { WA } \\ \mu \mathrm{g} / \mathrm{L} & 0 & \text { WA } \\ \mu \mathrm{g} / \mathrm{L} & 0 & \text { WA } \\ \mu \mathrm{g} / \mathrm{L} & 0 & \text { WA } \\ \mu \mathrm{g} / \mathrm{L} & 0 & \text { WA } \\ \mu \mathrm{g} / \mathrm{L} & 0 & \text { WA } \\ \mu \mathrm{g} / \mathrm{L} & 2 & \text { WA } \\ \mu \mathrm{g} / \mathrm{L} & 0 & \text { WA } \\ \mu \mathrm{g} / \mathrm{L} & 0 & \text { WA } \\ \mu \mathrm{g} / \mathrm{L} & 0 & \text { WA } \\ \mu \mathrm{g} / \mathrm{L} & 0 & \text { WA } \\ \mu \mathrm{g} / \mathrm{L} & 2 & \text { WA } \\ \mu \mathrm{g} / \mathrm{L} & 2 & \text { WA } \\ \mu \mathrm{g} / \mathrm{L} & 0 & \text { WA } \\ \mathrm{pC} \mathrm{i} / \mathrm{L} & 0 & \text { CN } \\ \mathrm{pC} \mathrm{i} / \mathrm{L} & 0 & \text { CN } \\ \mathrm{pCi} / \mathrm{L} & 0 & \text { CN } \\ \mathrm{pC} \mathrm{C} / \mathrm{mL} & 2 & \text { CN }\end{array}$

\section{WELL LFW 39}

$\begin{array}{llllll}\text { SRS Coord. } & \text { Lat/Longitude } & \text { Screen Zone Elevation } & \text { Top of Casing } & \text { Casing } & \text { Formation } \\ \text { N83213.1 } & 33.285220^{\circ} \mathrm{N} & 152.2-131.2 \mathrm{ft} \mathrm{msl} & 171.4 \mathrm{ft} \mathrm{msl} & 4 \text { " PVC } \\ \text { E46218.5 } & 81.706738^{\circ} \mathrm{W} & & & \end{array}$

\section{MEASUREMENTS CONDUCTED IN THE FIELD}

Sample date: 06/03/92

Depth to water: $28.25 \mathrm{ft}(8.61 \mathrm{~m})$ below TOC Water elevation: $143.15 \mathrm{ft}(43.63 \mathrm{~m}) \mathrm{msl}$

Sp. conductance: $31 \mu \mathrm{S} / \mathrm{cm}$

Water evacuated before sampling: $31 \mathrm{gal}$

\section{LABORATORY ANALYSES}

Time: $12: 00$

$\mathrm{pH}: 4.0$

Alkalinity: $0 \mathrm{mg} / \mathrm{L}$

Water temperature: $19.9^{\circ} \mathrm{C}$

\begin{tabular}{|c|c|c|c|c|}
\hline$\underline{H} \quad \underline{D}$ & Analyte & Result & Unit & Flag \\
\hline$\bullet$ & $\begin{array}{l}\mathrm{pH} \\
\text { Specific conductance } \\
\text { Aluminum } \\
\text { Antimony } \\
\text { Arsenic } \\
\text { Barium } \\
\text { Benzene } \\
\text { Bromodichloromethane }\end{array}$ & $\begin{aligned} & 5.1 \\
& 26 \\
& 25 \\
< & 2.6 \\
< & 2.0 \\
5.4 & < \\
< & 5.0 \\
< & 5.0\end{aligned}$ & $\begin{array}{l}\mathrm{pH} \\
\mu \mathrm{S} / \mathrm{cm} \\
\mu \mathrm{g} / \mathrm{L} \\
\mu \mathrm{g} / \mathrm{L} \\
\mu \mathrm{g} / \mathrm{L} \\
\mu \mathrm{g} / \mathrm{L} \\
\mu \mathrm{g} / \mathrm{L} \\
\mu \mathrm{g} / \mathrm{L}\end{array}$ & $\begin{array}{l}0 \\
0 \\
0 \\
0 \\
0 \\
0 \\
0 \\
0\end{array}$ \\
\hline
\end{tabular}

\footnotetext{
- = exceeded holding time. $\mathbf{a}=$ exceeded primary drinking water standard.
} 
WELL LFW 39 collected on 06/03/92, laboratory analyses (cont.)

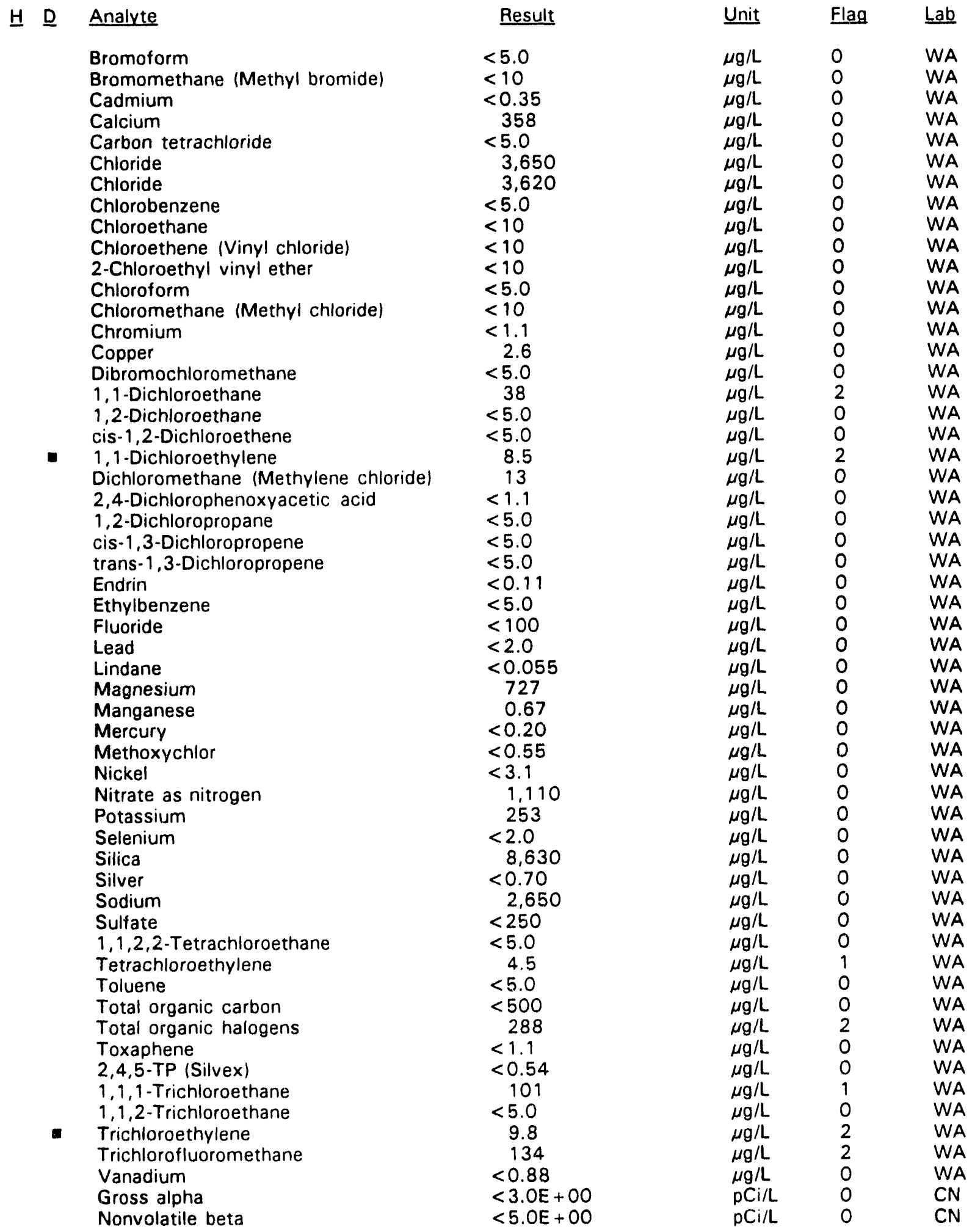

$\overline{0}=$ exceeded holding time. $=$ exceeded primary drinking water standard. 
WELL LFW 39 collected on 06/03/92, laboratory analyses (cont.)

H D Analyte

Radium-226

Tritium
Result

$<5.0 E-01$

$2.2 E+00 \pm 4.2 E-01$
Unit $\quad$ Flag $\underline{\text { Lab }}$

$\mathrm{pCi} / \mathrm{L} \quad \mathrm{O} \quad \mathrm{CN}$

$\mathrm{pCi} / \mathrm{mL} \quad \mathrm{O} \quad \mathrm{CN}$

\section{WELL LFW 40}

$\begin{array}{llllll}\text { SRS Coord. } & \text { Lat/Longitude } & \text { Screen Zone Elevation } & \text { Top of Casing } & \text { Casing } & \text { Formation } \\ \text { N83248.8 } & 33.285587^{\circ} \mathrm{N} & 152.2-131.2 \mathrm{ft} \mathrm{msl} & & 171.0 \mathrm{ft} \mathrm{msl} & \text { 4" PVC } \\ \text { E46395.1 } & 81.706343^{\circ} \mathrm{W} & & & \end{array}$

\section{MEASUREMENTS CONDUCTED IN THE FIELD}

Sample date: 06/03/92

Depth to water: $28.39 \mathrm{ft}(8.65 \mathrm{~m})$ below TOC

Water elevation: $142.61 \mathrm{ft}(43.47 \mathrm{~m}) \mathrm{msl}$

Sp. conductance: $17 \mu \mathrm{S} / \mathrm{cm}$

Water evacuated before sampling: $30 \mathrm{gal}$

\section{LABORATORY ANALYSES}

\begin{tabular}{|c|c|c|}
\hline$\underline{D}$ & nalyte & Result \\
\hline & $\begin{array}{l}\text { pH } \\
\text { Specific conductance } \\
\text { Aluminum } \\
\text { Antimony } \\
\text { Arsenic } \\
\text { Barium } \\
\text { Benzene } \\
\text { Bromodichloromethane } \\
\text { Bromoform } \\
\text { Bromomethane (Methyl bromide) } \\
\text { Cadmium } \\
\text { Calcium } \\
\text { Carbon tetrachloride } \\
\text { Chloride } \\
\text { Chlorobenzene } \\
\text { Chloroethane } \\
\text { Chloroethene (Vinyl chloride) } \\
\text { 2-Chloroethyl vinyl ether } \\
\text { Chloroform } \\
\text { Chloromethane (Methyl chloride) } \\
\text { Chromium } \\
\text { Copper } \\
\text { Dibromochloromethane } \\
\text { 1,1-Dichloroethane } \\
\text { 1,2-Dichloroethane } \\
\text { cis-1,2-Dichloroethene } \\
1,1-\text { Dichloroethylene } \\
\text { Dichloromethane (Methylene chloride) } \\
2,4-\text { Dichlorophenoxyacetic acid } \\
1,2-\text { Dichloropropane } \\
\text { cis-1,3-Dichloropropene } \\
\text { trans-1,3-Dichloropropene }\end{array}$ & $\begin{aligned} & 5.3 \\
& 16 \\
&< 15 \\
& 6.3 \\
&< 2.0 \\
&< 4.0 \\
&<5.0 \\
&<5.0 \\
&<5.0 \\
&<10 \\
&<0.35 \\
& \quad 290 \\
&<5.0 \\
& 2.610 \\
&<5.0 \\
&<10 \\
&<10 \\
&<10 \\
&<5.0 \\
&<10 \\
&<1.1 \\
& 2.8 \\
&<5.0 \\
&<5.0 \\
&<5.0 \\
&<5.0 \\
&<5.0 \\
& \quad 16 \\
&<1.1 \\
&<5.0 \\
&<5.0 \\
&<5.0\end{aligned}$ \\
\hline
\end{tabular}

Time: $11: 25$

$\mathrm{pH}: 4.3$

Alkalinity: $0 \mathrm{mg} / \mathrm{L}$

Water temperature: $19.7^{\circ} \mathrm{C}$

\begin{tabular}{|c|c|c|c|}
\hline Result & Unit & Flag & $\underline{\mathrm{Lab}}$ \\
\hline 5.3 & $\mathrm{pH}$ & 0 & WI \\
\hline 16 & $\mu \mathrm{S} / \mathrm{cm}$ & 0 & W \\
\hline 5 & $\mu \mathrm{g} / \mathrm{L}$ & 0 & $W_{i}$ \\
\hline 6.3 & $\mu \mathrm{g} / \mathrm{L}$ & 2 & $W_{1}$ \\
\hline 2.0 & $\mu \mathrm{g} / \mathrm{L}$ & 0 & $w_{1}$ \\
\hline 1.0 & $\mu \mathrm{g} / \mathrm{L}$ & 0 & WA \\
\hline 5.0 & $\mu \mathrm{g} / \mathrm{L}$ & 0 & WA \\
\hline 5.0 & $\mu g / L$ & 0 & WA \\
\hline 5.0 & $\mu \mathrm{g} / \mathrm{L}$ & 0 & WA \\
\hline 10 & $\mu \mathrm{g} / \mathrm{L}$ & 0 & WA \\
\hline 0.35 & $\mu \mathrm{g} / \mathrm{L}$ & 0 & W \\
\hline 290 & $\mu g / L$ & 0 & W \\
\hline 5.0 & $\mu \mathrm{g} / \mathrm{L}$ & 0 & W \\
\hline 2,610 & $\mu \mathrm{g} / \mathrm{L}$ & 0 & W \\
\hline 5.0 & $\mu g / L$ & 0 & W \\
\hline 10 & $\mu g / L$ & 0 & W \\
\hline 10 & $\mu \mathrm{g} / \mathrm{L}$ & 0 & W \\
\hline 10 & $\mu \mathrm{g} / \mathrm{L}$ & 0 & 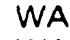 \\
\hline 5.0 & $\mu \mathrm{g} / \mathrm{L}$ & 0 & v \\
\hline 10 & $\mu \mathrm{g} / \mathrm{L}$ & 0 & WA \\
\hline 1.1 & $\mu \mathrm{g} / \mathrm{L}$ & 0 & W \\
\hline 2.8 & $\mu \mathrm{g} / \mathrm{L}$ & 0 & W \\
\hline 5.0 & $\mu \mathrm{g} / \mathrm{L}$ & 0 & W \\
\hline 5.0 & $\mu \mathrm{g} / \mathrm{L}$ & 0 & W \\
\hline 5.0 & $\mu \mathrm{g} / \mathrm{L}$ & 0 & W \\
\hline 0.0 & $\mu g / L$ & 0 & \\
\hline 5.0 & $\mu \mathrm{g} / \mathrm{L}$ & 0 & $W$ \\
\hline 16 & $\mu \mathrm{g} / \mathrm{L}$ & 0 & W \\
\hline 1.1 & $\mu \mathrm{g} / \mathrm{L}$ & 0 & W \\
\hline 5. & $\mu g / L$ & 0 & $w$ \\
\hline & $\mu \mathrm{g} / \mathrm{L}$ & 0 & $w$ \\
\hline 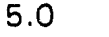 & $\mu \mathrm{g} / \mathrm{L}$ & 0 & \\
\hline
\end{tabular}

- exceeded holding time.

= exceeded primary drinking water standarü.

Sanitary Larıdfill 
WELL LFW 40 collected on 06/03/92, laboratory analyses (cont.)

\begin{tabular}{|c|c|c|c|c|}
\hline Analyte & Result & $\underline{\text { Unit }}$ & Flag & $\underline{L a b}$ \\
\hline Endrin & $<0.11$ & $\mu g / L$ & 0 & WA \\
\hline Ethylbenzene & $<5.0$ & $\mu g / L$ & 0 & WA \\
\hline Fluoride & $<100$ & $\mu \mathrm{g} / \mathrm{L}$ & 0 & WA \\
\hline Lead & $<2.0$ & $\mu \mathrm{g} / \mathrm{L}$ & 0 & WA \\
\hline Lindane & $<0.057$ & $\mu g / L$ & 0 & WA \\
\hline Magnesium & 354 & $\mu g / L$ & 0 & WA \\
\hline Manganese & $<0.35$ & $\mu \mathrm{g} / \mathrm{L}$ & 0 & WA \\
\hline Mercury & $<0.20$ & $\mu \mathrm{g} / \mathrm{L}$ & 0 & WA \\
\hline Methoxychlor & $<0.57$ & $\mu g / L$ & 0 & WA \\
\hline Nickel & $<3.1$ & $\mu g / L$ & 0 & WA \\
\hline Nitrate as nitrogen & 186 & $\mu \mathrm{g} / \mathrm{L}$ & 0 & WA \\
\hline Nitrate as nitrogen & 202 & $\mu g / L$ & 0 & WA \\
\hline Potassium & 350 & $\mu g / L$ & 0 & WA \\
\hline Selenium & $<2.0$ & $\mu \mathrm{g} / \mathrm{L}$ & 0 & WA \\
\hline Silica & 6,780 & $\mu g / L$ & 0 & WA \\
\hline Silver & 1.2 & $\mu \mathrm{g} / \mathrm{L}$ & 0 & WA \\
\hline Sodium & 2,000 & $\mu \mathrm{g} / \mathrm{L}$ & 0 & WA \\
\hline Sulfate & 407 & $\mu \mathrm{g} / \mathrm{L}$ & 0 & WA \\
\hline 1,1,2,2-Tetrachloroethane & $<5.0$ & $\mu \mathrm{g} / \mathrm{L}$ & 0 & WA \\
\hline Tetrachloroethylene & 1.5 & $\mu \mathrm{g} / \mathrm{L}$ & 0 & WA \\
\hline Toluene & $<5.0$ & $\mu g / L$ & 0 & WA \\
\hline Total organic carbon & $<500$ & $\mu g / L$ & 0 & WA \\
\hline Total organic halogens & 52 & $\mu \mathrm{g} / \mathrm{L}$ & 2 & WA \\
\hline Toxaphene & $<1.1$ & $\mu g / L$ & 0 & WA \\
\hline 2,4,5-TP (Silvex) & $<0.55$ & $\mu g / L$ & 0 & WA \\
\hline 1,1,1-Trichloroethane & 6.8 & $\mu \mathrm{g} / \mathrm{L}$ & 0 & WA \\
\hline 1,1,2-Trichloroethane & $<5.0$ & $\mu \mathrm{g} / \mathrm{L}$ & 0 & WA \\
\hline Trichloroethylene & 2.3 & $\mu \mathrm{g} / \mathrm{L}$ & 0 & WA \\
\hline Trichlorofluoromethane & 21 & $\mu g / L$ & 2 & WA \\
\hline Vanadium & $<0.88$ & $\mu \mathrm{g} / \mathrm{L}$ & 0 & WA \\
\hline Gross alpha & $<3 . \mathrm{OE}+\mathrm{OO}$ & $\mathrm{pCi} / \mathrm{L}$ & 0 & $\mathrm{CN}$ \\
\hline Nonvolatile beta & $<5.0 E+00$ & $\mathrm{pCi} / \mathrm{L}$ & 0 & $\mathrm{CN}$ \\
\hline Radium-226 & $<5$. OE-01 & $\mathrm{pCi} / \mathrm{L}$ & 0 & $\mathrm{CN}$ \\
\hline Tritium & $<2.0 E+00$ & $\mathrm{pCi} / \mathrm{mL}$ & 0 & $\mathrm{CN}$ \\
\hline
\end{tabular}

$\overline{0}=$ exceeded holding time. = exceeded primary drinking water standard. 
WELL LFW 41

$\begin{array}{llllll}\text { SRS Coord. } & \text { Lat/Longitude } & \text { Screen Zone Elevation } & \text { Top of Casing } & \text { Casing } & \text { Formation } \\ \text { N83304.9 } & 33.2860899^{\circ} \mathrm{N} & 151.3-130.3 \mathrm{ft} \mathrm{msl} & 170.5 \mathrm{ft} \mathrm{msl} & \text { 4" PVC } \\ \text { E46626.9 } & 81.705841^{\circ} \mathrm{W} & & & \end{array}$

\section{MEASUREMENTS CONDUCTED IN THE FIELD}

Sample date: 06/03/92

Depth to water: $26.44 \mathrm{ft}(8.06 \mathrm{~m})$ below TOC Water elevation: $144.06 \mathrm{ft}(43.91 \mathrm{~m}) \mathrm{ms}$ l

Sp. conductance: $15 \mu \mathrm{S} / \mathrm{cm}$

Water evacuated before sampling: $36 \mathrm{gal}$

\section{LABORATORY ANALYSES}

Time: $10: 45$

$\mathrm{pH}: 4.2$

Alkalinity: $0 \mathrm{mg} / \mathrm{L}$

Water temperature: $19.1^{\circ} \mathrm{C}$

\begin{tabular}{|c|c|c|c|c|c|}
\hline$\underline{H} \underline{D}$ & Analyte & Result & $\underline{\text { Unit }}$ & Flag & $\underline{L a b}$ \\
\hline : & $\begin{array}{l}\text { pH } \\
\text { Specific conductance } \\
\text { Aluminum } \\
\text { Antimony } \\
\text { Arsenic } \\
\text { Barium } \\
\text { Benzene } \\
\text { Bromodichloromethane } \\
\text { Bromoform } \\
\text { Bromomethane (Methyl bromide) } \\
\text { Cadmium } \\
\text { Calcium } \\
\text { Carbon tetrachloride } \\
\text { Chloride } \\
\text { Chlorobenzene } \\
\text { Chloroethane } \\
\text { Chloroethene (Vinyl chloride) } \\
\text { 2-Chloroethyl vinyl ether } \\
\text { Chloroform } \\
\text { Chloromethane (Methyl chloride) } \\
\text { Chromium } \\
\text { Copper } \\
\text { Dibromochloromethane } \\
\text { 1,1-Dichloroethane } \\
\text { 1,2-Dichloroethane } \\
\text { cis-1,2-Dichloroethene } \\
\text { 1,1-Dichloroethylene } \\
\text { Dichloromethane (Methylene chloride) } \\
2,4-\text { Dichlorophenoxyacetic acid } \\
\text { 1,2-Dichloropropane } \\
\text { cis-1,3-Dichloropropene } \\
\text { trans-1,3-Dichloropropene } \\
\text { Endrin } \\
\text { Ethylbenzene } \\
\text { Fluoride } \\
\text { Fluoride } \\
\text { Lead } \\
\text { Lindane } \\
\text { Magnesium }\end{array}$ & $\begin{aligned} & 5.3 \\
& 13 \\
&< 15 \\
& 6.0 \\
&< 2.0 \\
&< 4.0 \\
&< 5.0 \\
&< 5.0 \\
&< 5.0 \\
&< 10 \\
&< 0.35 \\
& 208 \\
&< 5.0 \\
& 2.450 \\
&< 5.0 \\
&< 10 \\
&< 10 \\
&< 10 \\
&<5.0 \\
&<10 \\
&<1.1 \\
&<1.1 \\
&<5.0 \\
&<5.0 \\
&<5.0 \\
&<5.0 \\
&<5.0 \\
&<5.0 \\
&<1.0 \\
&<5.0 \\
&<5.0 \\
&<5.0 \\
&<0.11 \\
&<5.0 \\
&<100 \\
&<100 \\
&<2.0 \\
&<0.054 \\
& \quad 289\end{aligned}$ & $\begin{array}{l}\operatorname{pH} \\
\mu \mathrm{S} / \mathrm{L} \\
\mu \mathrm{g} / \mathrm{L} \\
\mu \mathrm{g} / \mathrm{L} \\
\mu \mathrm{g} / \mathrm{L} \\
\mu \mathrm{g} / \mathrm{L} \\
\mu \mathrm{g} / \mathrm{L} \\
\mu \mathrm{g} / \mathrm{L} \\
\mu \mathrm{g} / \mathrm{L} \\
\mu \mathrm{g} / \mathrm{L} \\
\mu \mathrm{g} / \mathrm{L} \\
\mu \mathrm{g} / \mathrm{L} \\
\mu \mathrm{g} / \mathrm{L} \\
\mu \mathrm{g} / \mathrm{L} \\
\mu \mathrm{g} / \mathrm{L} \\
\mu \mathrm{g} / \mathrm{L} \\
\mu \mathrm{g} / \mathrm{L} \\
\mu \mathrm{g} / \mathrm{L} \\
\mu \mathrm{g} / \mathrm{L} \\
\mu \mathrm{g} / \mathrm{L} \\
\mu \mathrm{g} / \mathrm{L} \\
\mu \mathrm{g} / \mathrm{L} \\
\mu \mathrm{g} / \mathrm{L} \\
\mu \mathrm{g} / \mathrm{L} \\
\mu \mathrm{g} / \mathrm{L} \\
\mu \mathrm{g} / \mathrm{L} \\
\mu \mathrm{g} / \mathrm{L} \\
\mu \mathrm{g} / \mathrm{L} \\
\mu \mathrm{g} / \mathrm{L} \\
\mu \mathrm{g} / \mathrm{L}\end{array}$ & $\begin{array}{l}0 \\
0 \\
0 \\
2 \\
0 \\
0 \\
0 \\
0 \\
0 \\
0 \\
0 \\
0 \\
0 \\
0 \\
0 \\
0 \\
0 \\
0 \\
0 \\
0 \\
0 \\
0 \\
0 \\
0 \\
0 \\
0 \\
0 \\
0 \\
0 \\
0 \\
0 \\
0 \\
0 \\
0 \\
0 \\
0 \\
0 \\
0 \\
0\end{array}$ & $\begin{array}{l}\text { WA } \\
\text { WA } \\
\text { WA } \\
\text { WA } \\
\text { WA } \\
\text { WA } \\
\text { WA } \\
\text { WA } \\
\text { WA } \\
\text { WA } \\
\text { WA } \\
\text { WA } \\
\text { WA } \\
\text { WA } \\
\text { WA } \\
\text { WA } \\
\text { WA } \\
\text { WA } \\
\text { WA } \\
\text { WA } \\
\text { WA } \\
\text { WA } \\
\text { WA } \\
\text { WA } \\
\text { WA } \\
\text { WA } \\
\text { WA } \\
\text { WA } \\
\text { WA } \\
\text { WA } \\
\text { WA } \\
\text { WA } \\
\text { WA } \\
\text { WA } \\
\text { WA } \\
\text { WA } \\
\text { WA } \\
\text { WA } \\
\text { WA }\end{array}$ \\
\hline
\end{tabular}

$\overline{-}=$ exceeded holding time. $\mathbf{a}=$ exceeded primary drinking water standard. 
WELL LFW 41 collected on 06/03/92, laboratory analyses (cont.)

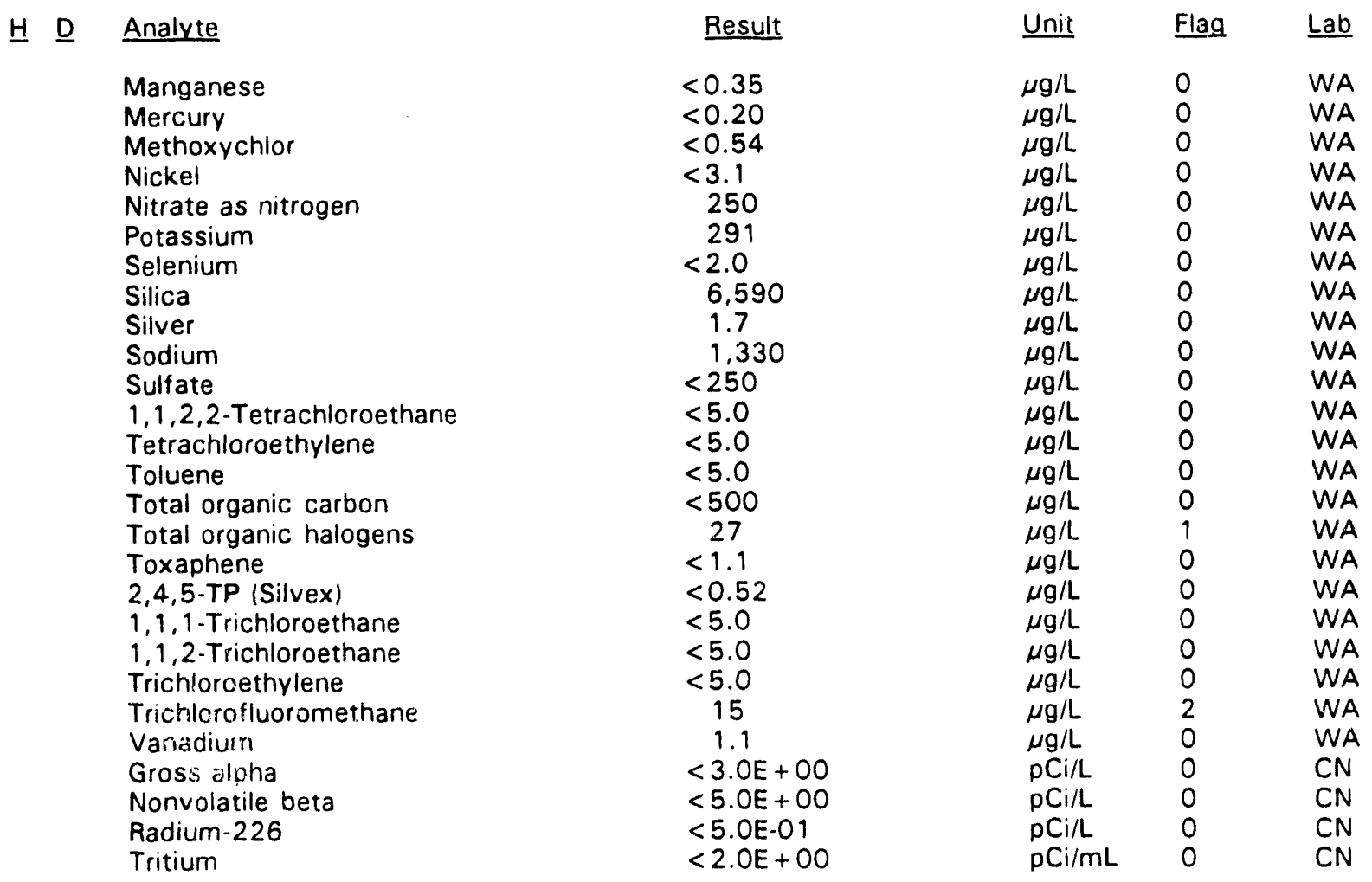

\section{WELL LFW 42}

$\begin{array}{llllll}\text { SRS Coord. } & \text { Lat/Longitude } & \text { Screen Zone Elevation } & \text { Top of Casing } & \text { Casing } & \text { Formation } \\ \text { N83776.2 } & 33.286978^{\circ} \mathrm{N} & 151.2-130.2 \mathrm{ft} \mathrm{msl} & 170.1 \mathrm{ft} \cdot \mathrm{msl} & 4 \text { "PVC } \\ \text { E46532.9 } & 81.707005^{\circ} \mathrm{W} & & & \end{array}$

MEASUREMENTS CONDUCTED IN THE FIELD

Sample date: 06/03/92

Depth to water: $23.65 \mathrm{ft}(7.21 \mathrm{~m})$ below TOC Water elevation: $146.45 \mathrm{ft}(44.64 \mathrm{~m}) \mathrm{msl}$

Sp. conductance: $15 \mu \mathrm{S} / \mathrm{cm}$

Water evacuated before sampling: $43 \mathrm{gal}$

\section{LABORATORY ANALYSES}

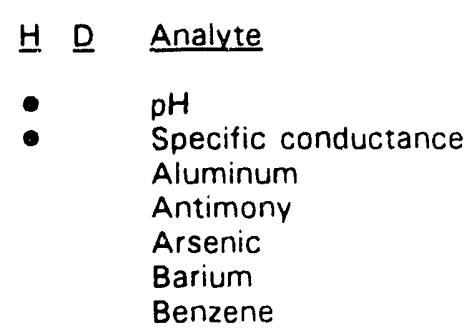

$\begin{aligned} & \text { Result } \\ & 5.4 \\ & 13 \\ < & 15 \\ < & 2.6 \\ < & 2.0 \\ < & 4.0 \\ < & 5.0\end{aligned}$

Time: 10:05

$\mathrm{pH}: 4.3$

Alkalinity: $0 \mathrm{mg} / \mathrm{L}$

Water temperature: $18.3^{\circ} \mathrm{C}$

\section{$c$}

$\begin{array}{lll}\text { Unit } & \text { Flag } & \text { Lab } \\ \mathrm{pH} & 0 & \text { WA } \\ \mu \mathrm{S} / \mathrm{cm} & 0 & \text { WA } \\ \mu \mathrm{g} / \mathrm{L} & 0 & \text { WA } \\ \mu \mathrm{g} / \mathrm{L} & 0 & \text { WA } \\ \mu \mathrm{g} / \mathrm{L} & 0 & \text { WA } \\ \mu \mathrm{g} / \mathrm{L} & 0 & \text { WA } \\ \mu \mathrm{g} / \mathrm{L} & 0 & \text { WA }\end{array}$

- = exceeded holding time. $\mathbf{m}=$ exceeded primary drinking water standard.

Sanitary Landfill 
WELL LFW 42 collected on 06/03/92, laboratory analyses (cont.)

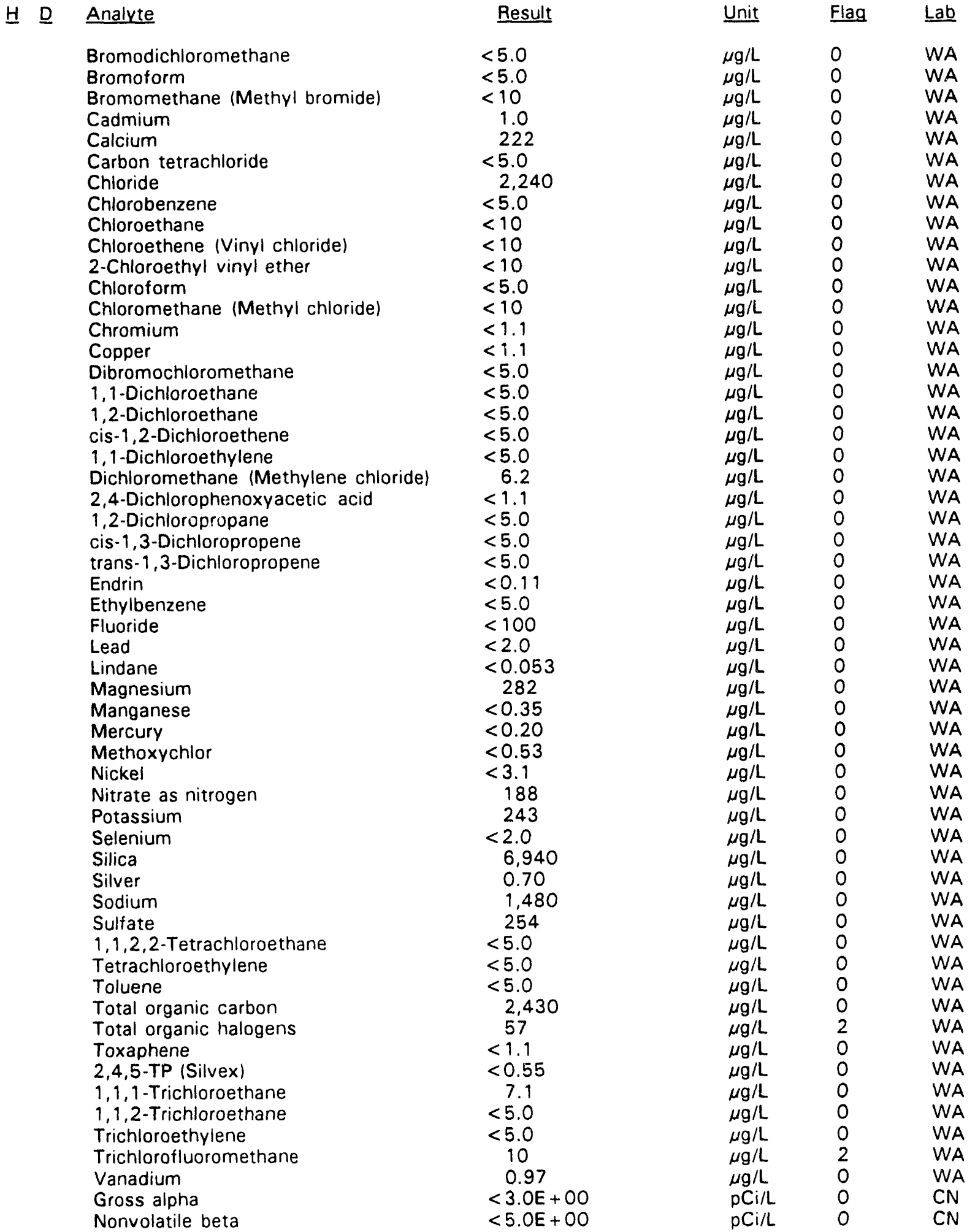

$\overline{- \text { = exceeded holding time. }} \mathbf{-}=$ exceeded primary drinking water standard. 
WELL LFW 42 collected on 06/03/92, laboratory analyses (cont.)

$\begin{array}{clllll}\text { H } & \text { Analyte } & \text { Result } & \text { Unit } & \text { Flag } & \underline{\text { Lab }} \\ \text { Radium-226 } & 8.1 \mathrm{E}-01 \pm 2.1 \mathrm{E}-01 & \mathrm{pCi} / \mathrm{L} & 0 & \mathrm{CN} \\ \text { Tritium } & <2.0 \mathrm{E}+00 & \mathrm{pCi} / \mathrm{mL} & 0 & \mathrm{CN}\end{array}$

\section{WELL. LFW 43B}

\begin{tabular}{|c|c|c|c|c|c|}
\hline SRS Coord. & Lat/Longitude & Screen Zone Elevation & Top of Casing & Casing & Formation \\
\hline $\begin{array}{l}\text { N86459.2 } \\
\text { E45240.5 }\end{array}$ & $\begin{array}{l}33.290800^{\circ} \mathrm{N} \\
81.715622^{\circ} \mathrm{W}\end{array}$ & $100.4-90.4 \mathrm{ft} \mathrm{msl}$ & $203.0 \mathrm{ft} \mathrm{msl}$ & 4" PVC & \\
\hline
\end{tabular}

\section{MEASUREMENTS CONDUCTED IN THE FIELD}

Sample date: 06/04/92

Depth to water: $37.49 \mathrm{ft}(11.43 \mathrm{~m})$ below TOC

Water elevation: $165.51 \mathrm{ft}(50.45 \mathrm{~m}) \mathrm{msl}$

Sp. conductance: $25 \mu \mathrm{S} / \mathrm{cm}$

Water evacuated before sampling: $198 \mathrm{gal}$

\section{LABORATORY ANALYSES}

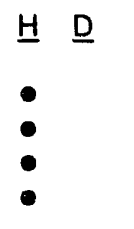

Analyte

$\mathrm{pH}$

$\mathrm{pH}$

Specific conductance

Specific conductance

Arsenic

Arsenic

Barium

Barium

Benzene

Bromodichloromethane

Bromoform

Bromomethane (Methyl bromide)

Cadmium

Cadmium

Calcium

Calcium

Carbon tetrachloride

Chloride

Chlorobenzene

Chloroethane

Chloroethene (Vinyl chloride)

2-Chloroethyl vinyl ether

Chloroform

Chioromethane (Methyl chloride)

Chromium

Chromium

Dibromochloromethane

1,1-Dichloroethane

1,2-Dichloroethane

cis-1,2-Dichloroethene

1,1-Dichloroethylene

Dichloromethane (Methylene chloride)

$\begin{array}{rl} & \text { Result } \\ & 5.6 \\ & 5.6 \\ 22 & 22 \\ 22 & <2.0 \\ < & 2.0 \\ & 6.8 \\ & 7.8 \\ <5.0 \\ <5.0 \\ <5.0 \\ <10 \\ 0.35 \\ 0.60 \\ 1.790 \\ 1.960 \\ <5.0 \\ 2.020 \\ <5.0 \\ <10 \\ <10 \\ <10 \\ <5.0 \\ <10 \\ <10 \\ <1.1 \\ <1.1 \\ <5.0 \\ <5.0 \\ <5.0 \\ <5.0 \\ <5.0 \\ 1.1 \\ <\end{array}$

Time: $11: 15$

$\mathrm{pH}: 5.3$

Alkalinity: $5 \mathrm{mg} / \mathrm{L}$

Water temperature: $18.8^{\circ} \mathrm{C}$ $\mathrm{ab}$

CN 
WELL LFW 43B collected on 06/04/92, laboratory analyses (cont.)

\section{H D Analyte}

2,4-Dichlorophenoxyacetic acid 1,2-Dichloropropane cis-1,3-Dichloropropene trans-1,3-Dichloropropene Endrin

Endrin

Ethylbenzene

Fluoride

Iron

Iron

Lead

Lead

Lindane

Lindane

Magnesium

Magnesium

Manganese

Manganese

Mercury

Methoxychlor

Methoxychlor

Methoxychlor

Nitrate as nitrogen

Phenols

Phenols

Potassium

Potassium

Selenium

Selenium

Silica

Silica

Silver

Silver

Sodium

Sodium

Sulfate

1,1,2,2-Tetrachloroethane

Tetrachloroethylene

Toluene

Total dissolved solids

Total organic carbon

Total organic halogens

Total organic halogens

Total phosphates (as P)

Toxaphene

Toxaphene

Toxaphene

2,4,5-TP (Silvex)

1,1,1-Trichloroethane

1,1,2-Trichloroethane

Trichloroethylene

Trichlorofluoromethane

Gross alpha

Nonvolatile beta

Radium-226

Tritium

\section{Result}

$<1.1$

$<5.0$

$<5.0$

$<5.0$

$<0.11$

$<0.22$

$<5.0$

$<100$

15

12

$<2.0$

$<2.0$

$<0.054$

$<0.11$

282

312

8.6

9.8

$<0.20$

$<0.54$

$<1.1$

$<1.1$

1.130

$<5.0$

$<5.0$

418

361

$<2.0$

$<2.0$

7,850

8,540

$<0.70$

$<0.70$

1,660

1,790

581

$<5.0$

$<5.0$

$<5.0$

62,000

$<500$

153

i. 2.7

65

$<1.1$

$<2.2$

$<2.2$

$<0.55$

$<5.0$

$<5.0$

$<5.0$

$<5.0$

$<3.0 E+00$

$<5.0 \mathrm{E}+00$

6.6E-01 $\pm 2.4 \mathrm{E}-01$

$<2.0 \mathrm{E}+00$

$\overline{-}=$ exceeded holding time.

= exceeded primary drinking water standard.

Sanitary Landfill 
WELL LFW 43C

$\begin{array}{lllllll}\text { SRS Coord. } & \text { Lat/Longitude } & \text { Screen Zone Elevation } & \text { Top of Casing } & \text { Casing } & \text { Formation } \\ \text { N86480.6 } & 33.290838^{\circ} \mathrm{N} & 138.5-128.5 \mathrm{ft} \mathrm{mst} & 202.6 \mathrm{ft} \mathrm{msl} & 4^{\prime \prime} \text { PVC } \\ \text { E45234.9 } & 81.715679{ }^{\circ} \mathrm{W} & & & \end{array}$

\section{MEASUREMENTS CONDUCTED IN THE FIELD}

Sample date: 06/04/92

Depth to water: $36.96 \mathrm{ft}(11.27 \mathrm{~m})$ below TOC Water elevation: $165.64 \mathrm{ft}(50.49 \mathrm{~m}) \mathrm{msl}$

Sp. conductance: $18 \mu \mathrm{S} / \mathrm{cm}$

Water evacuated before sampling: 99 gal

\section{LABORATORY ANALYSES}

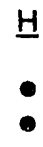

H D Analyte

$\mathrm{pH}$

Specific conductance

Arsenic

Barium

Benzene

Bromodichloromethane

Bromoform

Bromomethane (Methyl bromide)

Cadmium

Calcium

Carbon tetrachloride

Chloride

Chloride

Chlorobenzene

Chloroethane

Chloroethene (Vinyl chloride)

2-Chloroethyl vinyl ether

Chloroform

Chloromethane (Methyl chloride)

Chromium

Dibromochloromethane

1,1-Dichloroethane

1,2-Dichloroethane

cis-1,2-Dichloroethene

1,1-Dichloroethylene

Dichloromethane (Methylene chloride)

2,4-Dichlorophenoxyacetic acid

2,4-Dichlorophenoxyacetic acid

1,2-Dichloropropane

cis-1,3-Dichloropropene

trans-1,3-Dichloropropene

Endrin

Ethylbenzene

Fluoride

Iron

Lead

Lindane

Magnesium

Manganese
Time: $11: 40$

$\mathrm{pH}: 5.0$

Alkalinity: $1 \mathrm{mg} / \mathrm{L}$

Water temperature: $19.4^{\circ} \mathrm{C}$

\begin{tabular}{llll} 
Result & Unit & Flag & Lab \\
\hline 5.5 & $\mu \mathrm{HH}$ & 0 & WA \\
15 & $\mu \mathrm{S} / \mathrm{cm}$ & 0 & WA \\
$<2.0$ & $\mu \mathrm{g} / \mathrm{L}$ & 0 & WA \\
4.5 & $\mu \mathrm{g} / \mathrm{L}$ & 0 & WA \\
$<5.0$ & $\mu \mathrm{g} / \mathrm{L}$ & 0 & WA \\
$<5.0$ & $\mu \mathrm{g} / \mathrm{L}$ & 0 & WA \\
$<5.0$ & $\mu \mathrm{g} / \mathrm{L}$ & 0 & WA \\
$<10$ & $\mu \mathrm{g} / \mathrm{L}$ & 0 & WA \\
0.58 & $\mu \mathrm{g} / \mathrm{L}$ & 0 & WA \\
853 & $\mu \mathrm{g} / \mathrm{L}$ & 0 & WA \\
$<5.0$ & $\mu \mathrm{g} / \mathrm{L}$ & 0 & WA \\
1.730 & $\mu \mathrm{g} / \mathrm{L}$ & 0 & WA \\
1.730 & $\mu \mathrm{g} / \mathrm{L}$ & 0 & WA \\
$<5.0$ & $\mu \mathrm{g} / \mathrm{L}$ & 0 & WA \\
$<10$ & $\mu \mathrm{g} / \mathrm{L}$ & 0 & WA \\
$<10$ & $\mu \mathrm{g} / \mathrm{L}$ & 0 & WA \\
$<10$ & $\mu \mathrm{g} / \mathrm{L}$ & 0 & WA \\
$<5.0$ & $\mu \mathrm{g} / \mathrm{L}$ & 0 & WA \\
$<10$ & $\mu \mathrm{g} / \mathrm{L}$ & 0 & WA \\
$<1.1$ & $\mu \mathrm{g} / \mathrm{L}$ & 0 & WA \\
$<5.0$ & $\mu \mathrm{g} / \mathrm{L}$ & 0 & WA \\
$<5.0$ & $\mu \mathrm{g} / \mathrm{L}$ & 0 & WA \\
$<5.0$ & $\mu \mathrm{g} / \mathrm{L}$ & 0 & WA \\
$<5.0$ & $\mu \mathrm{g} / \mathrm{L}$ & 0 & WA \\
$<5.0$ & $\mu \mathrm{g} / \mathrm{L}$ & 0 & WA \\
$<5.0$ & $\mu \mathrm{g} / \mathrm{L}$ & 0 & WA \\
$<1.1$ & $\mu \mathrm{g} / \mathrm{L}$ & 0 & WA \\
$<2.2$ & $\mu \mathrm{g} / \mathrm{L}$ & 0 & WA \\
$<5.0$ & $\mu \mathrm{g} / \mathrm{L}$ & 0 & WA \\
$<5.0$ & $\mu \mathrm{g} / \mathrm{L}$ & 0 & WA \\
$<5.0$ & $\mu \mathrm{g} / \mathrm{L}$ & 0 & WA \\
$<0.11$ & $\mu \mathrm{g} / \mathrm{L}$ & 0 & WA \\
$<5.0$ & $\mu \mathrm{g} / \mathrm{L}$ & 0 & WA \\
$<100$ & $\mu \mathrm{g} / \mathrm{L}$ & 0 & WA \\
3.6 & $\mu \mathrm{g} / \mathrm{L}$ & 0 & WA \\
$<2.0$ & $\mu \mathrm{g} / \mathrm{L}$ & 0 & WA \\
$<0.054$ & $\mu \mathrm{g} / \mathrm{L}$ & 0 & WA \\
359 & $\mu \mathrm{g} / \mathrm{L}$ & 0 & WA \\
6.3 & $\mu \mathrm{g} / \mathrm{L}$ & 0 & WA \\
& & &
\end{tabular}

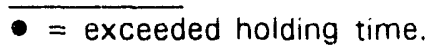

= exceeded primary drinking water standard. 
WELL LFW 43C collected on 06/04/92, laboratory analyses (cont.)

Analyte
Mercury
Methoxychlor
Nitrate as nitrogen
Phenols
Potassium
Selenium
Silica
Silver
Sodium
Sulfate
1,1,2,2-Tetrachloroethane
Tetrachloroethylene
Toluene
Total dissolved solids
Total organic carbon
Total organic halogens
Total phosphates (as P)
Toxaphene
2,4,5-TP (Silvex)
2,4,5-TP (Silvex)
1,1,1-Trichloroethane
1,1,2-Trichloroethane
Trichloroethylene
Trichlorofluoromethane
Gross alpha
Nonvolatile beta
Radium-226
Tritium

Result

$\begin{aligned} &< 0.20 \\ &< 0.54 \\ & 434 \\ &<5.0 \\ & 351 \\ &< 2.0 \\ & 6.480 \\ & 0.78 \\ & 1.020 \\ & 310 \\ &<5.0 \\ &<5.0 \\ &<5.0 \\ & 11.000 \\ &<500 \\ &<20 \\ & 48 \\ &< 1.1 \\ &< 0.55 \\ &< 1.1 \\ &<5.0 \\ &<5.0 \\ &<5.0 \\ &<5.0 \\ & 4.8 E+00 \pm 1.5 E+00 \\ &<5.0 E+00 \\ &<5.0 E-01 \\ &<2.0 E+00 \\ &<\end{aligned}$

\begin{tabular}{|c|c|}
\hline Unit & Flag \\
\hline$\mu \mathrm{g} / \mathrm{L}$ & 0 \\
\hline$\mu g / L$ & 0 \\
\hline$\mu \mathrm{g} / \mathrm{L}$ & 0 \\
\hline$\mu \mathrm{g} / \mathrm{L}$ & 0 \\
\hline$\mu g / L$ & 0 \\
\hline$\mu \mathrm{g} / \mathrm{L}$ & 0 \\
\hline$\mu g / L$ & 0 \\
\hline$\mu g / L$ & 0 \\
\hline$\mu g / L$ & 0 \\
\hline$\mu g / L$ & 0 \\
\hline$\mu g / L$ & 0 \\
\hline$\mu \mathrm{g} / \mathrm{L}$ & 0 \\
\hline$\mu \mathrm{g} / \mathrm{L}$ & 0 \\
\hline$\mu \mathrm{g} / \mathrm{L}$ & 0 \\
\hline$\mu \mathrm{g} / \mathrm{L}$ & 0 \\
\hline$\mu g / L$ & 0 \\
\hline$\mu \mathrm{g} / \mathrm{L}$ & 0 \\
\hline$\mu g / L$ & 0 \\
\hline$\mu g / L$ & 0 \\
\hline$\mu \mathrm{g} / \mathrm{L}$ & 0 \\
\hline$\mu \mathrm{g} / \mathrm{L}$ & 0 \\
\hline$\mu g / L$ & 0 \\
\hline$\mu \mathrm{g} / \mathrm{L}$ & 0 \\
\hline$\mu \mathrm{g} / \mathrm{L}$ & 0 \\
\hline $\mathrm{pCi} / \mathrm{L}$ & 0 \\
\hline $\mathrm{pCi} / \mathrm{L}$ & 0 \\
\hline $\begin{array}{c}\mathrm{Ci} / \mathrm{L} \\
\mathrm{Ci} / \mathrm{ml}\end{array}$ & $\begin{array}{l}0 \\
0\end{array}$ \\
\hline & \\
\hline
\end{tabular}

WELL LFW 43D

$\begin{array}{llllll}\text { SRS Coord. } & \text { Lat/Longitude } & \text { Screen Zone Elevation } & \text { Top of Casing } & \text { Casing } & \text { Formation } \\ \text { N86443.2 } & 33.290771^{\circ} \mathrm{N} & 170.9-150.9 \mathrm{ft} \mathrm{msl} & 202.9 \mathrm{ft} \mathrm{msl} & \text { A" PVC } & \\ \text { E45244.5 } & 81.715581^{\circ} \mathrm{W} & 17.9 & & \end{array}$

MEASUREMENTS CONDUCTED IN THE FIELD

Sample date: 06/04/92

Depth to water: $36.86 \mathrm{ft}(11.24 \mathrm{~m})$ below TOC

Water elevation: $166.04 \mathrm{ft}(50.61 \mathrm{~m}) \mathrm{ms}$ l

Sp. conductance: $19 \mu \mathrm{S} / \mathrm{cm}$

Water evacuated before sampling: $41 \mathrm{gal}$
Time: $10: 50$

pH: 4.9

Alkalinity: $0 \mathrm{mg} / \mathrm{L}$

Water temperature: $19.3^{\circ} \mathrm{C}$

\section{LABORATORY ANALYSES}

H D Analyte

:

$\mathrm{pH}$
Specific conductance

Arsenic

Barium

Benzene

Bromodichloromethane

$\begin{aligned} & \text { Resu } \\ & 5.3 \\ & 19 \\ &< \\ & 2.0 \\ & 10 \\ &<5.0 \\ &<5.0\end{aligned}$

$\begin{array}{llll}\text { Unit } & \text { Flag } & \text { Lab } \\ \mathrm{pH} & 0 & \text { WA } \\ \mu \mathrm{S} / \mathrm{cm} & 0 & \text { WA } \\ \mu \mathrm{g} / \mathrm{L} & 0 & \text { WA } \\ \mu \mathrm{g} / \mathrm{L} & 0 & \text { WA } \\ \mu \mathrm{g} / \mathrm{L} & 0 & \text { WA } \\ \mu \mathrm{g} / \mathrm{L} & 0 & \text { WA }\end{array}$

- = exceeded holding time.

- = exceeded primary drinking water standard.

Sanitary Landfill 
WELL LFW 43D collected on 06/04/92, laboratory analyses (cont.)

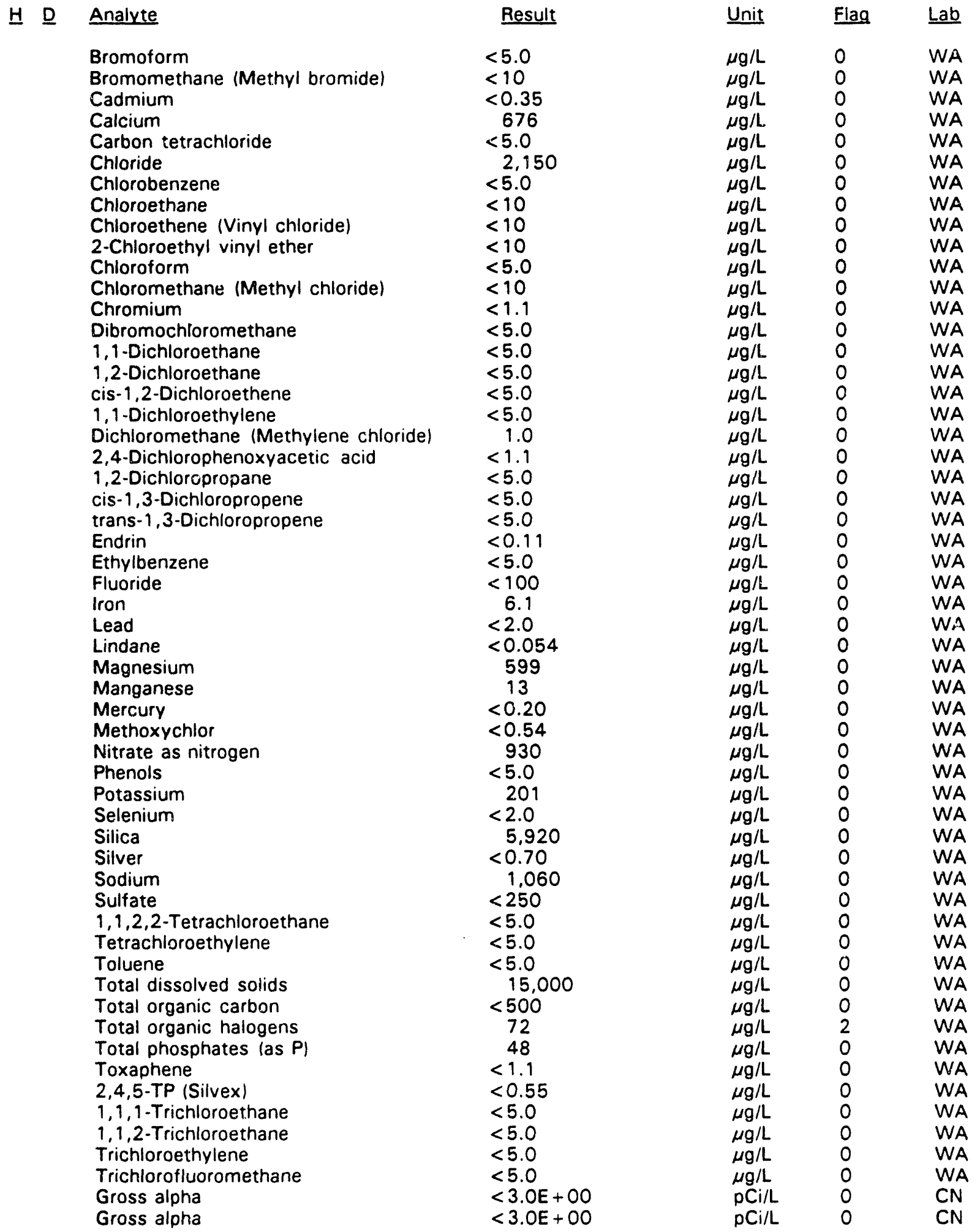

- exceeded hoiding time. = exceeded primary diinking vater standard. 
WELL LFW 43D collected on 06/04/92, laboratory analyses (cont.)

H D Analyte

Nonvolatile beta

Nonvolatile beta

Radium-226

Tritium

Tritium
Result

$$
\begin{aligned}
& <5.0 E+00 \\
& <5.0 E+00 \\
& <5.0 E-01 \\
& <2.0 E+00 \\
& <2.0 E+00
\end{aligned}
$$

Unit $\quad \underline{\text { Flag }} \quad \underline{\text { Lab }}$

$\mathrm{pCi} / \mathrm{L} \quad \mathrm{O} \quad \mathrm{CN}$

$\mathrm{pCi} / \mathrm{L} \quad 0 \quad \mathrm{CN}$

\begin{tabular}{|c|c|c|c|c|c|}
\hline SRS Coord. & Lat/Longitude & Screen Zone Elevation & Top of Casing & Casing & Formation \\
\hline $\begin{array}{l}\text { N84524.4 } \\
\text { E45022.6 }\end{array}$ & $\begin{array}{l}33.286167^{\circ} \mathrm{N} \\
81.712435^{\circ} \mathrm{W}\end{array}$ & $\mathrm{msl}$ & 170 & PVC & \\
\hline
\end{tabular}

$\mathrm{pCi} / \mathrm{L} \quad \mathrm{CN}$

$\mathrm{pCi} / \mathrm{mL} \quad \mathrm{C} \quad \mathrm{CN}$

$\mathrm{pCi} / \mathrm{mL} \quad 0 \quad \mathrm{CN}$

\section{WELL LFW 44D}

MEASUREMENTS CONDUCTED IN THE FIELD

Sample date: 06/04/92

Depth to water: $15.95 \mathrm{ft}(4.86 \mathrm{~m})$ below TOC Water elevation: $154.35 \mathrm{ft}(47.05 \mathrm{~m}) \mathrm{msl}$

Sp. conductance: $21 \mu \mathrm{S} / \mathrm{cm}$

Water evacuated before sampling: $39 \mathrm{gal}$

\section{LABORATORY ANALYSES}

Time: $14: 45$

pH: 4.7

Alkalinity: $0 \mathrm{mg} / \mathrm{L}$

Water temperature: $19.4^{\circ} \mathrm{C}$

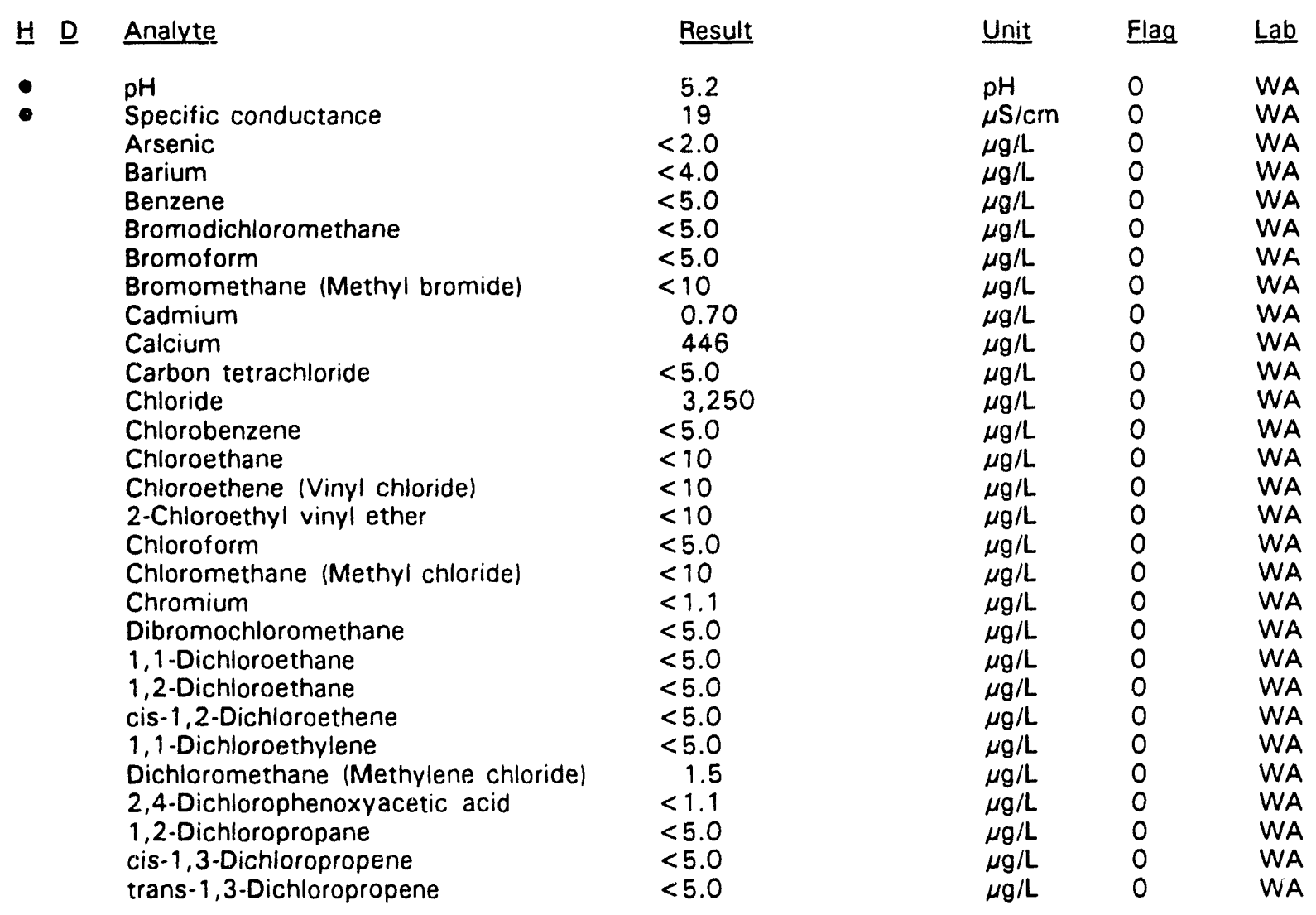

$\overline{- \text { = exceeded holding time. }} \mathbf{-}=$ exceeded primary drinking water standard. 
WELL LFW 44D collected on 06/04/92, laboratory analyses (cont.)

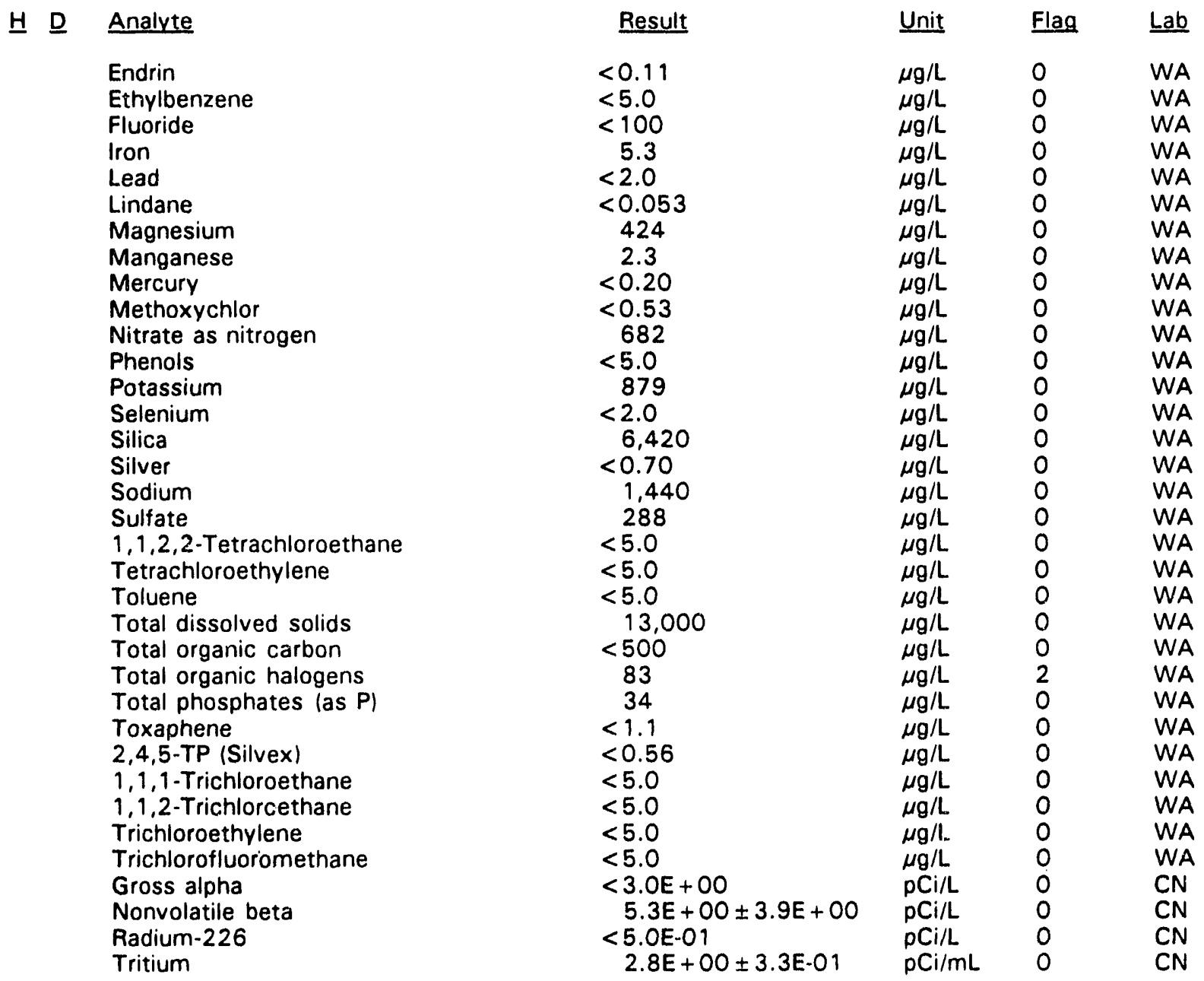

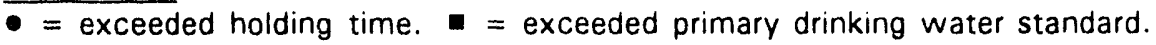


WELL LFW 45D

$\begin{array}{llllll}\text { SRS Coord. } & \text { Lat/Longitude } & \text { Screen Zone Elevation } & \text { Top of Casing } & \text { Casing } & \text { Formation } \\ \text { N84217.8 } & 33.285684^{\circ} \mathrm{N} & 154.7-134.7 \mathrm{ft} \mathrm{msl} & 166.3 \mathrm{ft} \mathrm{msl} & \text { 4" PVC } \\ \text { E45142.0 } & 81.711525^{\circ} \mathrm{W} & & & \end{array}$

MEASUREMENTS CONDUCTED IN THE FIELD

Sample date: 06/05/92

Depth to water: $14.53 \mathrm{ft}(4.43 \mathrm{~m})$ below TOC Water elevation: $151.77 \mathrm{ft}(46.26 \mathrm{~m}) \mathrm{msl}$

Sp. conductance: $19 \mu \mathrm{S} / \mathrm{cm}$

Water evacuated before sampling: 45 gal

\section{LABORATORY ANALYSES}

H D Anaiyte

pH
pH
pH
pH
pH
pH
Specific conductance
Specific conductance
Specific conductance
Specific conductance
Specific conductance
Specific conductance
Arsenic
Arsenic
Arsenic
Arsenic
Arsenic
Barium
Barium
Barium
Barium
Barium
Benzene
Benzene
Benzene
Benzene
Benzene
Bromodichloromethane
Bromodichloromethane
Bromodichloromethane
Bromodichloromethane
Bromodichloromethane
Bromodichloromethane
Bromoform
Bromoform
Bromoform
Bromoform
Bromoform
Bromoform

?

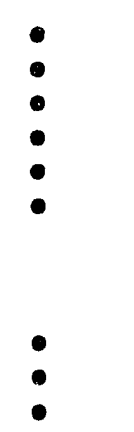

Time: $10: 00$

pH: 4.4

Alkalinity: $0 \mathrm{mg} / \mathrm{L}$

Water temperature: $18.9^{\circ} \mathrm{C}$ 
WELL LFW 45D collected on 06/05/92, laboratory analyses (cont.)

\section{H D Analyte}

Bromomethane (Methyl bromide)

Bromomethane (Methyl bromide)

Bromomethane (Methyl bromide)

Bromomethane (Methyl bromide)

Bromomethane (Methyl bromide)

Bromomethane (Methyl bromide)

Cadmium

Cadmium

Cadmium

Cadmium

Cadmium

Calcium

Calcium

Calcium

Calcium

Calcium

Carbon tetrachloride

Carbon tetrachloride

Carbon tetrachloride

Carbon tetrachloride

Carbon tetrachloride

Carbon tetrachloride

Chloride

Chloride

Chloride

Chloride

Chloride

Chlorobenzene

Chlorobenzene

Chlorobenzene

Chlorobenzene

Chlorobenzene

Chloroethane

Chloroethane

Chloroethane

Chloroethane

Chloroethane

Chloroethane

Chloroethene (Vinyl chloride)

Chloroethene (Vinyl chloride)

Chloroethene (Vinyl chloride)

Chloroethene (Vinyl chloride)

Chloroethene (Vinyl chloride)

Chloroethene (Vinyl chloride)

2-Chloroethyl vinyl ether

2-Chloroethyl vinyl ether

2-Chloroethyl vinyl ether

2-Chloroethyl vinyi ether

2-Chioroethyl vinyl ether

2-Chloroethyl vinyl ether

Chloroform

Chloroform

Chloroform

Chloroform

Chloroform

Chloroform

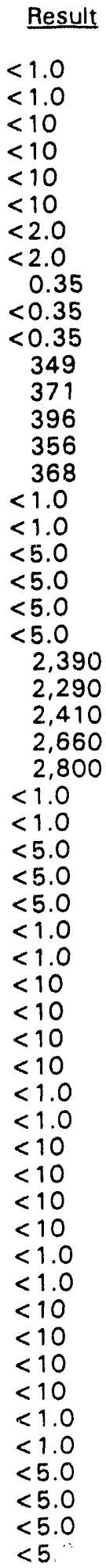

\begin{tabular}{|c|c|c|}
\hline Unit & Flag & $\underline{L a b}$ \\
\hline$\mu \mathrm{g} / \mathrm{L}$ & 0 & $\mathrm{GE}$ \\
\hline$\mu \mathrm{g} / \mathrm{L}$ & 0 & GE \\
\hline$\mu \mathrm{g} / \mathrm{L}$ & 0 & WA \\
\hline$\mu g / L$ & 0 & WA \\
\hline$\mu \mathrm{g} / \mathrm{L}$ & 0 & WA \\
\hline$\mu g / L$ & 0 & WA \\
\hline$\mu \mathrm{g} / \mathrm{L}$ & 0 & GE \\
\hline$\mu \mathrm{g} / \mathrm{L}$ & 0 & GE \\
\hline$\mu \mathrm{g} / \mathrm{L}$ & 0 & WA \\
\hline$\mu \mathrm{g} / \mathrm{L}$ & 0 & WA \\
\hline$\mu \mathrm{g} / \mathrm{L}$ & 0 & WA \\
\hline$\mu \mathrm{g} / \mathrm{L}$ & 0 & GE \\
\hline$\mu g / L$ & 0 & GE \\
\hline$\mu \mathrm{g} / \mathrm{L}$ & 0 & WA \\
\hline$\mu \mathrm{g} / \mathrm{L}$ & 0 & WA \\
\hline$\mu \mathrm{g} / \mathrm{L}$ & 0 & WA \\
\hline$\mu g / L$ & 0 & GE \\
\hline$\mu \mathrm{g} / \mathrm{L}$ & 0 & GE \\
\hline$\mu \mathrm{g} / \mathrm{L}$ & 0 & WA \\
\hline$\mu \mathrm{g} / \mathrm{L}$ & 0 & WA \\
\hline$\mu \mathrm{g} / \mathrm{L}$ & 0 & WA \\
\hline$\mu \mathrm{g} / \mathrm{L}$ & 0 & WA \\
\hline$\mu g / L$ & 0 & GE \\
\hline$\mu \mathrm{g} / \mathrm{L}$ & 0 & $\mathrm{GE}$ \\
\hline$\mu g / L$ & 0 & GE \\
\hline$\mu g / L$ & 0 & WA \\
\hline$\mu g / L$ & 0 & WA \\
\hline$\mu g / L$ & 0 & GE \\
\hline$\mu \mathrm{g} / \mathrm{L}$ & 0 & GE \\
\hline$\mu \mathrm{g} / \mathrm{L}$ & 0 & WA \\
\hline$\mu \mathrm{g} / \mathrm{L}$ & 0 & WA \\
\hline$\mu \mathrm{g} / \mathrm{L}$ & 0 & WA \\
\hline$\mu \mathrm{g} / \mathrm{L}$ & 0 & GE \\
\hline$\mu g / L$ & 0 & GE \\
\hline$\mu g / L$ & 0 & WA \\
\hline$\mu g / L$ & 0 & WA \\
\hline$\mu g / L$ & 0 & WA \\
\hline$\mu \mathrm{g} / \mathrm{L}$ & 0 & WA \\
\hline$\mu g / L$ & 0 & GE \\
\hline$\mu \mathrm{g} / \mathrm{L}$ & 0 & GE \\
\hline$\mu g / L$ & 0 & WA \\
\hline$\mu g / L$ & 0 & WA \\
\hline$\mu g / L$ & 0 & WA \\
\hline$\mu \mathrm{g} / \mathrm{L}$ & 0 & WA \\
\hline$\mu \mathrm{g} / \mathrm{L}$ & 0 & GE \\
\hline$\mu \mathrm{g} / \mathrm{L}$ & 0 & GE \\
\hline$\mu g / L$ & 0 & WA \\
\hline$\mu g / L$ & 0 & WA \\
\hline$\mu g / L$ & 0 & WA \\
\hline$\mu \mathrm{g} / \mathrm{L}$ & 0 & WA \\
\hline$\mu \mathrm{g} / \mathrm{L}$ & 0 & GE \\
\hline$\mu \mathrm{g} / \mathrm{L}$ & 0 & GE \\
\hline$\mu \mathrm{g} / \mathrm{L}$ & 0 & WA \\
\hline$\mu g / L$ & 0 & WA \\
\hline$\mu \mathrm{g} / \mathrm{L}$ & 0 & WA \\
\hline$\mu \mathrm{g} / \mathrm{L}$ & 0 & \\
\hline
\end{tabular}

$\overline{- \text { = exceeded holding time. }}$ = exceeded primary drinking water standard. 
WELL LFW 45D collected on 06/05/92, laboratory analyses (cont.)

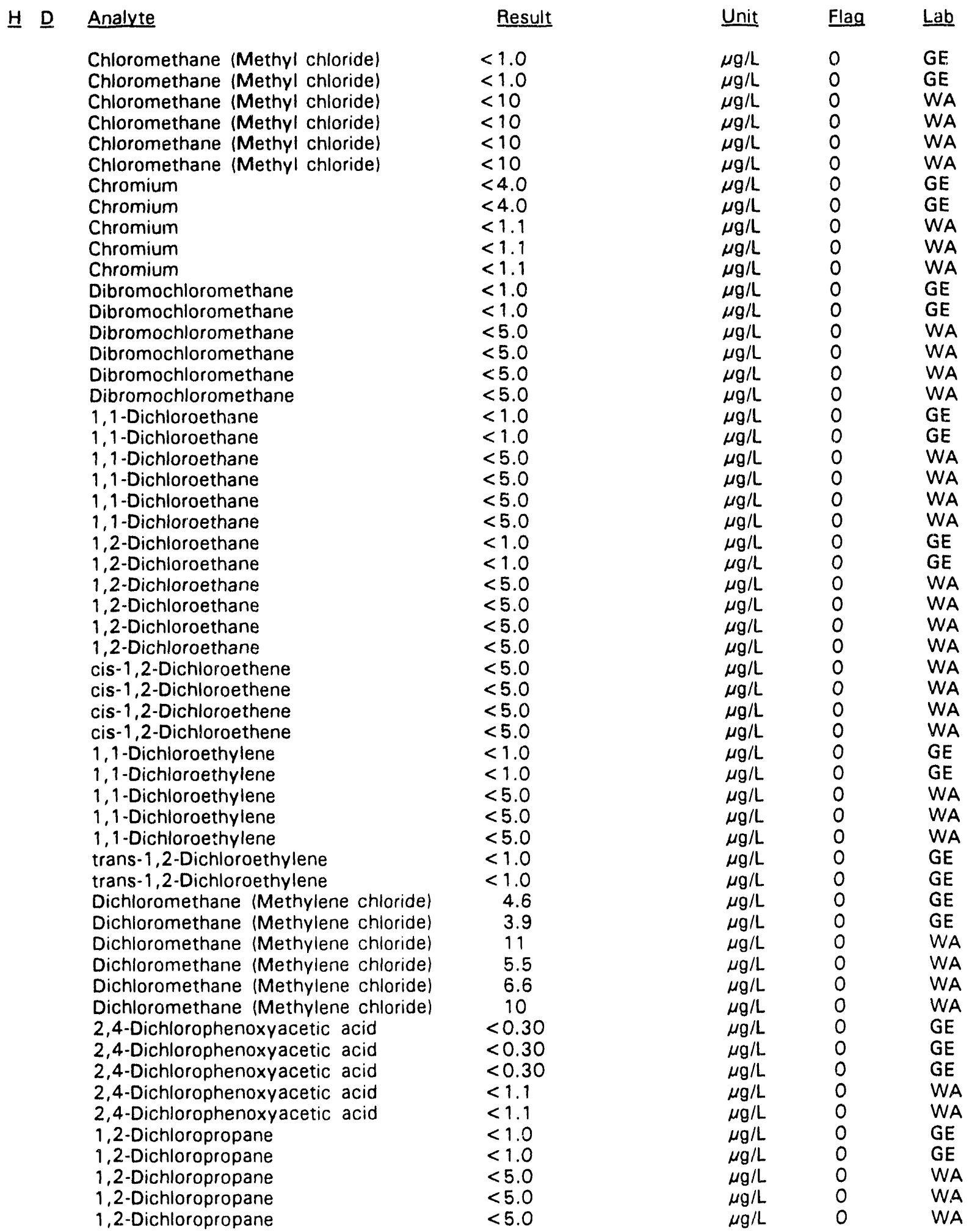

$\overline{- \text { = exceeded holding time. }}$ - = exceeded primary drinking water standard. 
WELL LFW 45D collected on 06/05/92, laboratory analyses (cont.)

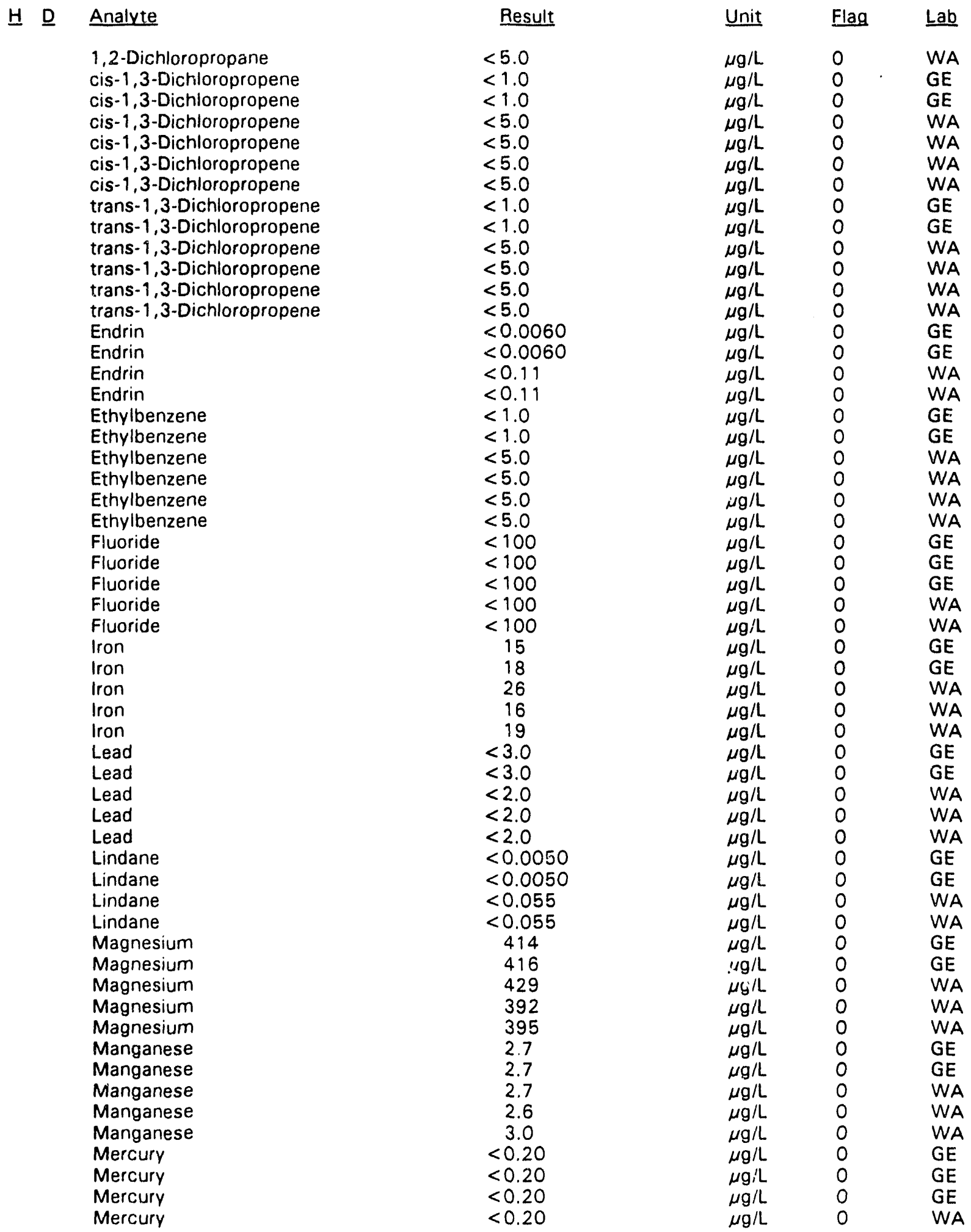

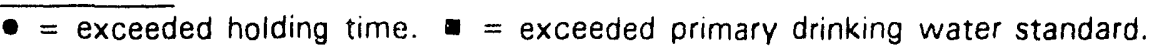


WELL LFW 45D collected on 06/05/92, laboratory analyses (cont.)

\begin{tabular}{|c|c|c|c|c|}
\hline Analyte & Result & Unit & Flag & Lab \\
\hline Mercury & $<0.20$ & $\mu g / L$ & 0 & WA \\
\hline Methoxychlor & $<0.50$ & $\mu \mathrm{g} / \mathrm{L}$ & 0 & GE \\
\hline Methoxychlor & $<0.50$ & $\mu \mathrm{g} / \mathrm{L}$ & 0 & $\mathrm{GE}$ \\
\hline Methoxychlor & $<0.55$ & $\mu \mathrm{g} / \mathrm{L}$ & 0 & WA \\
\hline Methoxychlor & $<0.55$ & $\mu \mathrm{g} / \mathrm{L}$ & 0 & WA \\
\hline Nitrate as nitrogen & 100 & $\mu \mathrm{g} / \mathrm{L}$ & 0 & GE \\
\hline Nitrate as nitrogen & 100 & $\mu g / L$ & 0 & GE \\
\hline Nitrate as nitrogen & 325 & $\mu \mathrm{g} / \mathrm{L}$ & 0 & WA \\
\hline Nitrate as nitrogen & 335 & $\mu \mathrm{g} / \mathrm{L}$ & 0 & WA \\
\hline Nitrate as nitrogen & 291 & $\mu g / L$ & 0 & WA \\
\hline Phenols & $<5.0$ & $\mu \mathrm{g} / \mathrm{L}$ & 0 & GE \\
\hline Phenols & $<5.0$ & $\mu \mathrm{g} / \mathrm{L}$ & 0 & GE \\
\hline Phenols & $<5.0$ & $\mu g / L$ & 0 & WA \\
\hline Phenols & $<5.0$ & $\mu \mathrm{g} / \mathrm{L}$ & 0 & WA \\
\hline Phenols & $<5.0$ & $\mu g / L$ & 0 & WA \\
\hline Potassium & 556 & $\mu \mathrm{g} / \mathrm{L}$ & 0 & GE \\
\hline Potassium & 597 & $\mu \mathrm{g} / \mathrm{L}$ & 0 & $\mathrm{GE}$ \\
\hline Potassium & 569 & $\mu \mathrm{g} / \mathrm{L}$ & 0 & WA \\
\hline Potassium & 569 & $\mu g / L$ & 0 & WA \\
\hline Potassium & 452 & $\mu \mathrm{g} / \mathrm{L}$ & 0 & WA \\
\hline Selenium & $<2.0$ & $\mu g / L$ & 0 & GE \\
\hline Selenium & $<2.0$ & $\mu g / L$ & 0 & GE \\
\hline Selenium & $<2.0$ & $\mu g / L$ & 0 & WA \\
\hline Selenium & $<2.0$ & $\mu \mathrm{g} / \mathrm{L}$ & 0 & WA \\
\hline Selenium & $<2.0$ & $\mu \mathrm{g} / \mathrm{L}$ & 0 & WA \\
\hline Silica & 6,630 & $\mu \mathrm{g} / \mathrm{L}$ & 0 & GE \\
\hline Silica & 6,660 & $\mu \mathrm{g} / \mathrm{L}$ & 0 & GE \\
\hline Silica & 5,470 & $\mu \mathrm{g} / \mathrm{L}$ & 0 & WA \\
\hline Silica & 5,230 & $\mu \mathrm{g} / \mathrm{L}$ & 0 & WA \\
\hline Silica & 5,210 & $\mu \mathrm{g} / \mathrm{L}$ & 0 & WA \\
\hline Silver & $<2.0$ & $\mu \mathrm{g} / \mathrm{L}$ & 0 & GE \\
\hline Silver & $<2.0$ & $\mu \mathrm{g} / \mathrm{L}$ & 0 & GE \\
\hline Silver & $<0.70$ & $\mu \mathrm{g} / \mathrm{L}$ & 0 & WA \\
\hline Silver & $<0.70$ & $\mu \mathrm{g} / \mathrm{L}$ & 0 & WA \\
\hline Silver & $<0.70$ & $\mu \mathrm{g} / \mathrm{L}$ & 0 & WA \\
\hline Sodium & 1,210 & $\mu g / L$ & 0 & GE \\
\hline Sodium & 1,220 & $\mu \mathrm{g} / \mathrm{L}$ & 0 & GE \\
\hline Sodium & 1,290 & $\mu \mathrm{g} / \mathrm{L}$ & 0 & WA \\
\hline Sodium & 1,230 & $\mu \mathrm{g} / \mathrm{L}$ & 0 & WA \\
\hline Sodium & 1,210 & $\mu \mathrm{g} / \mathrm{L}$ & 0 & WA \\
\hline Sulfate & $<1,000$ & $\mu g / L$ & 0 & GE \\
\hline Sulfate & $<1,000$ & $\mu g / L$ & 0 & GE \\
\hline Sulfate & $<1,000$ & $\mu \mathrm{g} / \mathrm{L}$ & 0 & GE \\
\hline Sulfate & 484 & $\mu \mathrm{g} / \mathrm{L}$ & 0 & WA \\
\hline Sulfate & 464 & $\mu \mathrm{g} / \mathrm{L}$ & 0 & WA \\
\hline Sulfate & 780 & $\mu g / L$ & 0 & WA \\
\hline $1,1,2,2 \cdot$ Tetrachloroethane & $<1.0$ & $\mu \mathrm{g} / \mathrm{L}$ & 0 & GE \\
\hline $1,1,2,2$-Tetrachloroethane & $<1.0$ & $\mu \mathrm{g} / \mathrm{L}$ & 0 & GE \\
\hline $1,1,2,2$-Tetrachloroethane & $<5.0$ & $\mu \mathrm{g} / \mathrm{L}$ & 0 & WA \\
\hline $1,1,2,2$-Tetrachloroethane & $<5.0$ & $\mu \mathrm{g} / \mathrm{L}$ & 0 & WA \\
\hline $1,1,2,2$-Tetrachloroethane & $<5.0$ & $\mu \mathrm{g} / \mathrm{L}$ & 0 & WA \\
\hline $1,1,2,2$-Tetrachloroethane & $<5.0$ & $\mu \mathrm{g} / \mathrm{L}$ & 0 & WA \\
\hline Tetrachloroethylene & $<1.0$ & $\mu \mathrm{g} / \mathrm{L}$ & 0 & GE \\
\hline Tetrachloroethylene & 2.9 & $\mu g / L$ & 1 & GE \\
\hline Tetrachloroethylene & $<5.0$ & $\mu \mathrm{g} / \mathrm{L}$ & 0 & WA \\
\hline Tetrachloroethylene & $<5.0$ & $\mu \mathrm{g} / \mathrm{L}$ & 0 & WA \\
\hline
\end{tabular}

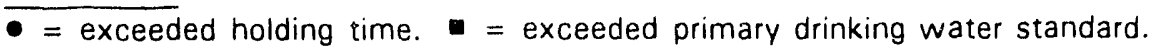


WELL LFW 45D collected on 06/05/92, laboratory analyses (cont.)

H D Analyte

Tetrachloroethylene

Tetrachloroethylene

Toluene

Toluene

Toluene

Toluene

Toluene

Total dissolved solids

Total dissolved solids

Total dissolved solids

Total dissolved solids

Total dissolved solids

Total organic carbon

Total organic carbon

Total organic carbon

Total organic carbon

Total organic halogens

Total organic halogens

Total organic halogens

Total organic halogens

Total phosphates (as P

Total phosphates (as P)

Total phosphates (as P)

Total phosphates (as P)

Toxaphene

Toxaphene

Toxaphene

Toxaphene

2,4,5-TP (Silvex)

2,4,5-TP (Silvex)

2,4,5-TP (Silvex)

2,4,5-TP (Silvex)

2,4,5-TP (Silvex)

$1,1,1$-Trichloroethane

1,1,1-Trichloroethane

1,1,1-Trichloroethane

1,1,1-Trichloroethane

1,1,1-Trichloroethane

1,1,1-Trichloroethane

1,1,2-Trichloroethane

1,1,2-Trichloroethane

1,1,2-Trichloroethane

1,1,2-Trichloroethane

$1,1,2$-Trichloroethane

1,1,2-Trichloroethane

Trichloroethylene

Trichloroethylene

Trichloroethylene

Trichloroethylene

Trichloroethylene

Trichlorofluoromethane

Trichlorofluoromethane

Trichlorufluoromethane

Trichlorofluoromethane

Trichlorofluoromethane

Trichlorofluoromethane
Result

$<5.0$

$<5.0$

$<1.0$

$<1.0$

$<5.0$

$<5.0$

$<5.0$

7,000

8,000

15,000

11,000

13,000

$<1,000$

$<1,000$

$<500$

$<500$

$<5.0$

5.2

37

$<10$

$<50$

$<50$

62

$<20$

$<0.24$

$<0.24$

$<1.1$

$<1.1$

$<0.090$

$<0.090$

$<0.090$

$<0.54$

$<0.55$

$<1.0$

$<1.0$

$<5.0$

$<5.0$

$<5.0$

$<5.0$

$<1.0$

$<1.0$

$<5.0$

$<5.0$

$<5.0$

$<5.0$

$<1.0$

1.5

$<5.0$

$<5.0$

$<5.0$

$<1.0$

$<1.0$

$<5.0$

$<5.0$

$<5.0$

$<5.0$
Unit Flag Lab

$\mu g / L \quad 0 \quad$ WA

$\mu g / L \quad 0 \quad$ WA

$\mu g / L \quad 0 \quad$ GE

$\mu g / L \quad 0 \quad$ GE

$\mu g / L \quad O \quad W A$

$\mu g / L \quad 0 \quad$ WA

$\mu g / L \quad 0 \quad$ WA

$\mu g / L \quad 0 \quad$ GE

$\mu \mathrm{g} / \mathrm{L} \quad \mathrm{O} \quad \mathrm{GE}$

$\mu \mathrm{g} / \mathrm{L} \quad 0 \quad$ WA

WA

$\mu \mathrm{g} / \mathrm{L} \quad 0 \quad$ WA

$\mu \mathrm{g} / \mathrm{L} \quad \mathrm{O} \quad \mathrm{GE}$

$\mu \mathrm{g} / \mathrm{L} \quad 0 \quad \mathrm{GE}$

$\mu \mathrm{g} / \mathrm{L} \quad 0 \quad$ WA

$\mu \mathrm{g} / \mathrm{L} \quad 0 \quad$ WA

$\mu g / L \quad 0 \quad$ GE

$\mu g / L \quad 0 \quad$ GE

$\mu \mathrm{g} / \mathrm{L} \quad 1 \quad$ WA

$\mu g / L \quad O \quad W A$

$\mu \mathrm{g} / \mathrm{L} \quad \mathrm{O} \quad \mathrm{GE}$

$\mu g / L \quad 0 \quad$ GE

$\mu g / L \quad 0 \quad$ WA

$\mu g / L \quad 0 \quad$ WA

$\mu g / L \quad 0 \quad$ GE

$\mu g / L \quad 0 \quad$ GE

$\mu g / L \quad 0 \quad$ WA

$\mu g / L \quad 0 \quad$ WA

$\mu \mathrm{g} / \mathrm{L} \quad 0 \quad$ GE

$\mu g / L \quad 0 \quad G E$

$\mu g / L \quad 0 \quad$ GE

$\mu \mathrm{g} / \mathrm{L} \quad 0 \quad$ WA

$\mu g / L \quad 0 \quad$ WA

$\mu g / L \quad 0 \quad$ GE

$\mu g / L \quad 0 \quad$ GE

$\mu \mathrm{g} / \mathrm{L} \quad 0 \quad$ WA

$\mu g / L \quad$ WA

$\mu g / L \quad 0 \quad W A$

$\mu \mathrm{g} / \mathrm{L} \quad 0 \quad$ WA

$\mu \mathrm{g} / \mathrm{L} . \quad 0 \quad \mathrm{GE}$

$\mu \mathrm{g} / \mathrm{L} \quad \mathrm{O} \quad \mathrm{GE}$

$\mu g / L \quad O \quad W A$

$\mu g / L \quad 0 \quad$ WA

$\mu g / L \quad 0 \quad W A$

$\mu \mathrm{g} / \mathrm{L} \quad \mathrm{O} \quad$ WA

$\mu g / L$

$\mu g / L$

$\mu g / L$

$\mu g / L$

$\mu g / L$

$\mu \mathrm{g} / \mathrm{L}$

$\mu g / L$

$\mu \mathrm{g} / \mathrm{L}$

$\mu \mathrm{g} / \mathrm{L}$

$\mu \mathrm{g} / \mathrm{L}$

$\mu \mathrm{g} / \mathrm{L}$

$$
\text { GE }
$$

GE

WA

WA

WA

GE

$G E$

WA

WA

WA

WA $\overline{0}=$ exceeded holding time.

Sanitary Landfil! 
WELL LFW 45D collected on 06/05/92, laboratory analyses (cont.)

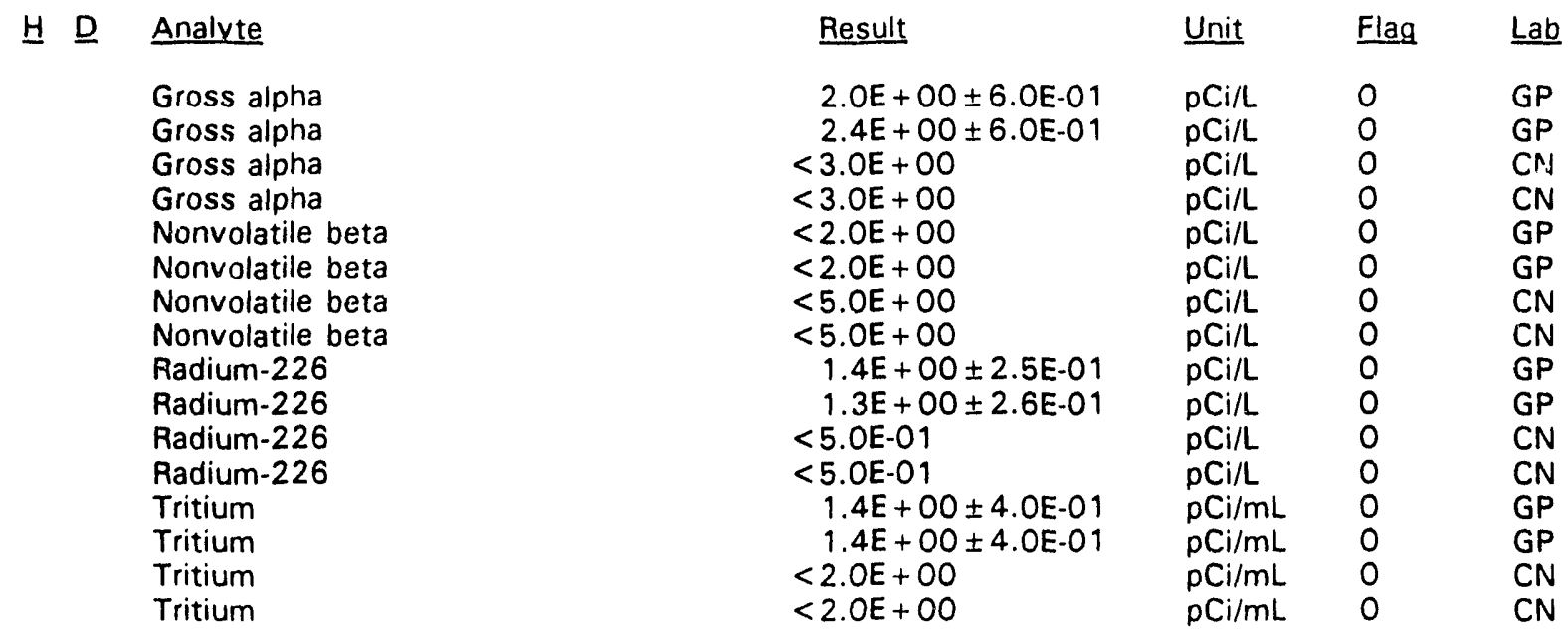

\section{WELL LFW 46D}

\begin{tabular}{|c|c|c|c|c|c|}
\hline SRS Coord. & Lat/Longitude & Screen Zone Elevation & Top of Casing & Casing & Formation \\
\hline $\begin{array}{l}\text { N84054.0 } \\
E 45162.8\end{array}$ & $\begin{array}{l}33.285356^{\circ} \mathrm{N} \\
81.711152^{\circ} \mathrm{W}\end{array}$ & 157 & $\mathrm{msl}$ & VC & \\
\hline
\end{tabular}

MEASUREMENTS CONDUCTED IN THE FIELD

Sample date: 06/04/92

Depth to water: $14.59 \mathrm{ft}(4.45 \mathrm{~m})$ below TOC

Water elevation: $150.51 \mathrm{ft}(45.88 \mathrm{~m}) \mathrm{msl}$

Sp. conductance: $110 \mu \mathrm{S} / \mathrm{cm}$

Water evacuated before sampling: $35 \mathrm{gal}$

\section{LABORATORY ANALYSES}

H D Analyte

$\mathrm{pH}$

Specific conductance

Arsenic

Barium

Benzene

Bromodichloromethane

Bromoform

Bromomethane (Methyl bromide)

Cadmium

Calcium

Carbon tetrachloride

Chloride

Chlorobenzene

Chloroethane

Chloroethene (Vinyl chloride)

2-Chloroethyl vinyl ether

Chloroform

Chloromethane (Methyl chloride)
Time: $15: 25$

pH: 5.3

Alkalinity: $4 \mathrm{mg} / \mathrm{L}$

Water temperature: $18.9^{\circ} \mathrm{C}$ ab
GP
GP
$C N$
$C N$
GP
GP
$C N$
$C N$
GP
GP
$C N$
$C N$
GP
GP
$C N$
$C N$ 
WELL LFW 46D collected on 06/04/92, laboratory analyses (cont.)

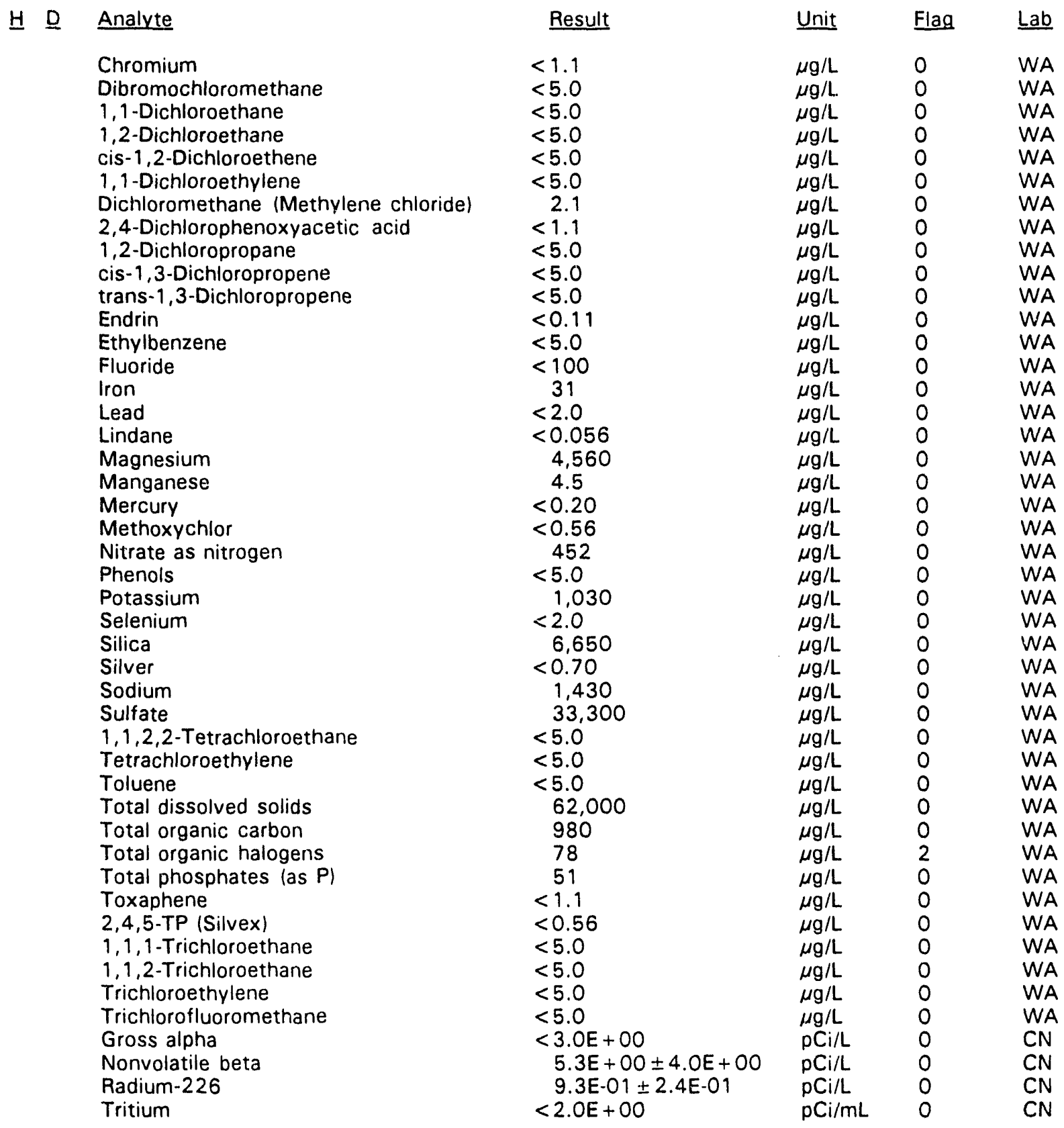

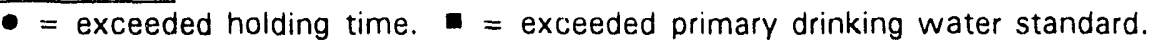


WELL LFW 47C

$\begin{array}{llllll}\text { SRS Coord. Lat/Longitude } & \text { Screen Zone Elevation } & \text { Top of Casing } & \text { Casing } & \text { Formation } \\ \text { N83843.9 } & 33.284917^{\circ} \mathrm{N} & 115.8-105.7 \mathrm{ft} \mathrm{msl} & 161.4 \mathrm{ft} \mathrm{msl} & \text { 4" PVC } \\ \text { E45178.6 } & 81.710702^{\circ} \mathrm{W} & & & \end{array}$

\section{MEASUREMENTS CONDUCTED IN THE FIELD}

Sample date: 06/04/92

Depth to water: $13.16 \mathrm{ft}(4.01 \mathrm{~m})$ below TOC Water elevation: $148.24 \mathrm{ft}(45.18 \mathrm{~m}) \mathrm{msl}$

Sp. conductance: $64 \mu \mathrm{S} / \mathrm{cm}$

Water evacuated before sampling: $112 \mathrm{gal}$

\section{LABORATORY ANALYSES}

\section{H}

:

\section{$\mathrm{pH}$}

Specific conductance

Arsenic

Barium

Benzene

Bromodichloromethane

Bromoform

Bromomethane (Methyl bromide)

Cadmium

Calcium

Carbon tetrachloride

Chloride

Chlorobenzene

Chloroethane

- Chloroethene (Vinyl chloride)

2-Chloroethyl vinyl ether

Chloroform

Chloromethane (Methyl chloride)

Chromium

Dibromochloromethane

1,1-Dichloroethane

1,2-Dichloroethane

cis-1,2-Dichloroethene

1,1-Dichloroethylene

Dichloromethane (Methylene chloride)

2,4-Dichlorophenoxyacetic acid

1,2-Dichloropropane

cis-1,3-Dichloropropene

trans-1,3-Dichloropropene

Endrin

Ethylbenzene

Fluoride

Iron

Lead

Lindane

Magnesium

Manganese

Mercury

Methoxychlor

\section{Result}

\section{8}

5.8
56

$<2.0$

$<4.0$

$<5.0$

$<5.0$

$<5.0$

$<10$

0.60

1,520

$<5.0$

2.250

$<5.0$

$<10$

7.7

$<10$

$<5.0$

$<10$

$<1.1$

$<5.0$

2.0

$<5.0$

$<5.0$

$<5.0$

2.1

$<1.1$

$<5.0$

$<5.0$

$<5.0$

$<0.11$

$<5.0$

$<100$

5.2

$<2.0$

$<0.053$

3,100

3.8

$<0.20$

$<0.53$
Time: $16: 25$

pH: 5.5

Alkalinity: $10 \mathrm{mg} / \mathrm{L}$

Water temperature: $19.3^{\circ} \mathrm{C}$ 
WELL LFW 47C collected on 06/04/92, laboratory analyses (cont.)

\begin{tabular}{|c|c|c|c|c|c|}
\hline \multirow[t]{26}{*}{$\underline{H} \quad \underline{D}$} & Analyte & Result & Unit & Flag & $\underline{\text { Lab }}$ \\
\hline & Nitrate as nitrogen & 543 & $\mu \mathrm{g} / \mathrm{L}$ & 0 & WA \\
\hline & Phenols & $<5.0$ & $\mu g / L$ & 0 & WA \\
\hline & Potassium & 788 & $\mu g / L$ & 0 & WA \\
\hline & Selenium & $<2.0$ & $\mu g / L$ & 0 & WA \\
\hline & Silica & 7,570 & $\mu \mathrm{g} / \mathrm{L}$ & 0 & WA \\
\hline & Silver & $<0.70$ & $\mu \mathrm{g} / \mathrm{L}$ & 0 & WA \\
\hline & Sodium & 4,470 & $\mu \mathrm{g} / \mathrm{L}$ & 0 & WA \\
\hline & Sulfate & 290 & $\mu \mathrm{g} / \mathrm{L}$ & 0 & WA \\
\hline & $1,1,2,2$-Tetrachloroethane & $<5.0$ & $\mu \mathrm{g} / \mathrm{L}$ & 0 & WA \\
\hline & Tetrachloroethylene & $<5.0$ & $\mu g / L$ & 0 & WA \\
\hline & Toluene & $<5.0$ & $\mu \mathrm{g} / \mathrm{L}$ & 0 & WA \\
\hline & Total dissolved solids & 32,000 & $\mu \mathrm{g} / \mathrm{L}$ & 0 & WA \\
\hline & Total organic carbon & 877 & $\mu \mathrm{g} / \mathrm{L}$ & 0 & WA \\
\hline & Total organic halogens & 107 & $\mu \mathrm{g} / \mathrm{L}$ & 2 & WA \\
\hline & Total phosphates (as P) & 22 & $\mu \mathrm{g} / \mathrm{L}$ & 0 & WA \\
\hline & Toxaphene & $<1.1$ & $\mu \mathrm{g} / \mathrm{L}$ & 0 & WA \\
\hline & 2,4,5-TP (Silvex) & $<0.56$ & $\mu g / L$ & 0 & WA \\
\hline & $1,1,1$-Trichloroethane & $<5.0$ & $\mu \mathrm{g} / \mathrm{L}$ & 0 & WA \\
\hline & 1,1,2-Trichloroethane & $<5.0$ & $\mu g / L$ & 0 & WA \\
\hline & Trichloroethylene & $<5.0$ & $\mu g / L$ & 0 & WA \\
\hline & Trichlor ofluoromethane & $<5.0$ & $\mu \mathrm{g} / \mathrm{L}$ & 0 & WA \\
\hline & Gross alpha & $<3.0 E+00$ & $\mathrm{pCi} / \mathrm{L}$ & 0 & $\mathrm{CN}$ \\
\hline & Nonvolatile beta & $<5.0 E+00$ & $\mathrm{pCi} / \mathrm{L}$ & 0 & $\mathrm{CN}$ \\
\hline & Radium-226 & $9.9 \mathrm{E}-01 \pm 2.2 \mathrm{E}-01$ & $\mathrm{pCi} / \mathrm{L}$ & 0 & $\mathrm{CN}$ \\
\hline & Tritium & $3.4 E+00 \pm 3.5 E-01$ & $\mathrm{pCi} / \mathrm{mL}$ & 0 & $\mathrm{CN}$ \\
\hline
\end{tabular}

\section{WELL LFW 47D}

$\begin{array}{llllll}\text { SRS Coord. } & \text { Lat/Longitude } & \text { Screen Zone Elevation } & \text { Top of Casing } & \text { Casing } & \text { Formation } \\ \text { N83859.3 } & 33.284933^{\circ} \mathrm{N} & 154.7-134.9 \mathrm{ft} \mathrm{msl} & & 161.7 \mathrm{ft} \mathrm{msl} & \text { 4" PVC } \\ \text { E45167.9 } & 81.710760^{\circ} \mathrm{W} & & & \end{array}$

MEASUREMENTS CONDUCTED IN THE FIELD

Sample date: 06/04/92

Depth to water: $12.91 \mathrm{ft}(3.94 \mathrm{~m})$ below TOC

Water elevation: $148.79 \mathrm{ft}(45.35 \mathrm{~m} / \mathrm{msl}$

Sp. conductance: $29 \mu \mathrm{S} / \mathrm{cm}$

Water evacuated before sampling: $36 \mathrm{gal}$

\section{LABORATORY ANALYSES}

브 Analyte

$\begin{array}{ll}\text { - } & \mathrm{pH} \\ \text { Specific conductance }\end{array}$

Arsenic

Barium

Benzene

Bromodichloromethane

Bromoform

Bromomethane (Methyl bromide)

Cadmium
Time: 16:05

$\mathrm{pH}: 5.2$

Alkalinity: $1 \mathrm{mg} / \mathrm{L}$

Water temperature: $19.5^{\circ} \mathrm{C}$ 
WELL LFW 470 collected on 06/04/92, laboratory analyses (cont.)

H D Analyte

Calcium

Carbon tetrachloride

Chloride

Chlorobenzene

Chloroethane

Chloroethene (Vinyl chloride)

2-Chloroethyl vinyl ether

Chloroform

Chloromethane (Methyl chloride)

Chromium

Dibromochloromethane

1,1-Dichloroethane

1,2-Dichloroethane

cis-1,2-Dichloroethene

1,1-Dichloroethylene

Dichloromethane (Methylene chloride)

2,4-Dichlorophenoxyacetic acid

1,2-Dichloropropane

cis-1,3-Dichloropropene

trans-1,3-Dichloropropene

Endrin

Ethylbenzene

Fiuuride

Fluoride

Iron

Lead

Lindane

Magnesium

Manganese

Mercury

Methoxychlor

Nitrate as nitrogen

Phenols

Potassium

Selenium

Silica

Silver

Sodium

Sulfate

1,1,2,2-Tetrachloroethane

Tetrachloroethylene

Toluene

Total dissolved solids

Total organic carbon

Total organic halogens

Total phosphates (as P)

Toxaphene

2,4,5-TP (Silvex)

1,1,1-Trichloroethane

1,1,2-Trichloroethane

Trichloroethylene

Trichlorofluoromethane

Gross alpha

Nonvolatile beta

Radium-226

Tritium

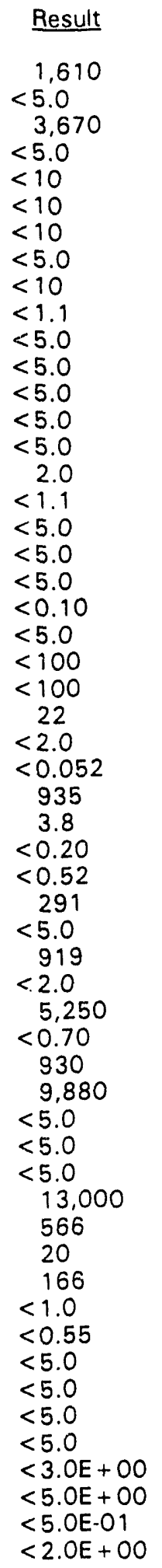

\begin{tabular}{|c|c|c|}
\hline Unit & Flag & $\underline{L a b}$ \\
\hline$\mu g / L$ & 0 & WA \\
\hline$\mu g / L$ & 0 & WA \\
\hline$\mu g / L$ & 0 & WA \\
\hline$\mu \mathrm{g} / \mathrm{L}$ & 0 & WA \\
\hline$\mu \mathrm{g} / \mathrm{L}$ & 0 & WA \\
\hline$\mu \mathrm{g} / \mathrm{L}$ & 0 & WA \\
\hline$\mu \mathrm{g} / \mathrm{L}$ & 0 & WA \\
\hline$\mu \mathrm{g} / \mathrm{L}$ & 0 & WA \\
\hline$\mu g / L$ & 0 & WA \\
\hline$\mu g / L$ & 0 & WA \\
\hline$\mu \mathrm{g} / \mathrm{L}$ & 0 & WA \\
\hline$\mu \mathrm{g} / \mathrm{L}$ & 0 & WA \\
\hline$\mu \mathrm{g} / \mathrm{L}$ & 0 & WA \\
\hline$\mu g / L$ & 0 & WA \\
\hline$\mu g / L$ & 0 & WA \\
\hline$\mu \mathrm{g} / \mathrm{L}$ & 0 & WA \\
\hline$\mu \mathrm{g} / \mathrm{L}$ & 0 & WA \\
\hline$\mu g / L$ & 0 & WA \\
\hline$\mu g / L$ & 0 & WA \\
\hline$\mu \mathrm{g} / \mathrm{L}$ & 0 & WA \\
\hline$\mu g / L$ & 0 & WA \\
\hline$\mu \mathrm{g} / \mathrm{L}$ & 0 & WA \\
\hline$\mu g / L$ & 0 & WA \\
\hline$\mu \mathrm{g} / \mathrm{L}$ & 0 & WA \\
\hline$\mu \mathrm{g} / \mathrm{L}$ & 0 & WA \\
\hline$\mu g / L$ & 0 & WA \\
\hline$\mu \mathrm{g} / \mathrm{L}$ & 0 & WA \\
\hline$\mu \mathrm{g} / \mathrm{L}$ & 0 & WA \\
\hline$\mu g / L$ & 0 & WA \\
\hline$\mu \mathrm{g} / \mathrm{L}$ & 0 & WA \\
\hline$\mu \mathrm{g} / \mathrm{L}$ & 0 & WA \\
\hline$\mu \mathrm{g} / \mathrm{L}$ & 0 & WA \\
\hline$\mu g / L$ & 0 & WA \\
\hline$\mu g / L$ & 0 & WA \\
\hline$\mu \mathrm{g} / \mathrm{L}$ & 0 & WA \\
\hline$\mu \mathrm{g} / \mathrm{L}$ & 0 & WA \\
\hline$\mu \mathrm{g} / \mathrm{L}$ & 0 & WA \\
\hline$\mu \mathrm{g} / \mathrm{L}$ & 0 & WA \\
\hline$\mu \mathrm{g} / \mathrm{L}$ & 0 & WA \\
\hline$\mu \mathrm{g} / \mathrm{L}$ & 0 & WA \\
\hline$\mu \mathrm{g} / \mathrm{L}$ & 0 & WA \\
\hline$\mu \mathrm{g} / \mathrm{L}$ & 0 & WA \\
\hline$\mu \mathrm{g} / \mathrm{L}$ & 0 & WA \\
\hline$\mu \mathrm{g} / \mathrm{L}$ & 0 & WA \\
\hline$\mu \mathrm{g} / \mathrm{L}$ & 0 & WA \\
\hline$\mu \mathrm{g} / \mathrm{L}$ & 0 & WA \\
\hline$\mu \mathrm{g} / \mathrm{L}$ & 0 & WA \\
\hline$\mu \mathrm{g} / \mathrm{L}$ & 0 & WA \\
\hline$\mu \mathrm{g} / \mathrm{L}$ & 0 & WA \\
\hline$\mu \mathrm{g} / \mathrm{L}$ & 0 & WA \\
\hline$\mu \mathrm{g} / \mathrm{L}$ & 0 & WA \\
\hline$\mu \mathrm{g} / \mathrm{L}$ & 0 & WA \\
\hline pCi/L & 0 & $\mathrm{CN}$ \\
\hline $\mathrm{pCi} / \mathrm{L}$ & 0 & $\mathrm{CN}$ \\
\hline $\mathrm{pCi} / \mathrm{L}$ & 0 & $\mathrm{CN}$ \\
\hline $\mathrm{pCi} /$ & 0 & $\mathrm{CN}$ \\
\hline
\end{tabular}

- = exceeded holding time.

- = exceeded primary drinking water standard. 
WELL LFW 48C

\begin{tabular}{|c|c|c|c|c|c|}
\hline SRS Coord. & Lat/Longitude & Screen Zone Elevation & Top of Casing & $\underline{\text { Casing }}$ & Formation \\
\hline $\begin{array}{l}\text { N83877.0 } \\
\text { E45430.3 }\end{array}$ & $\begin{array}{l}33.285401^{\circ} \mathrm{N} \\
81.710103^{\circ} \mathrm{W}\end{array}$ & $118.2-10$ & $169.3 \mathrm{ft} \mathrm{msl}$ & 4" PVC & \\
\hline
\end{tabular}

MEASUREMENTS CONDUCTED IN THE FIELD

Sample date: 06/04/92

Depth to water: $21.04 \mathrm{ft}(6.41 \mathrm{~m})$ below TOC

Water elevation: $148.26 \mathrm{ft}(45.19 \mathrm{~m}) \mathrm{msl}$

Sp. conductance: $27 \mu \mathrm{S} / \mathrm{cm}$

Water evacuated before sampling: $105 \mathrm{gal}$

\section{LABORATORY ANALYSES}

H D Analyte

$\mathrm{pH}$

Specific conductance

Arsenic

Barium

Benzene

Bromodichloromethane

Bromoform

Bromomethane (Methyl bromide)

Cadmium

Calcium

Carbon tetrachloride

Chloride

Chlorobenzene

Chloroethane

Chloroethene (Vinyl chloride)

2-Chloroethyl vinyl ether

Chloroform

Chloromethane (Methyl chloride)

Chromium

Dibromochloromethane

1,1-Dichloroethane

1,2-Dichloroethane

cis-1,2-Dichloroethene

1,1-Dichloroethylene

Dichloromethane (Methylene chloride)

2,4-Dichlorophenoxyacetic acid

1,2-Dichloropropane

cis-1,3-Dichloropropene

trans-1,3-Dichloropropene

Endrin

Ethylbenzene

Fluoride

Iron

Lead

Lindane

Magnesium

Manganese

Mercury

Methoxychlor
Result

5.6

19
$<2.0$

8.7

$<5.0$

$<5.0$

$<5.0$

$<10$

$<0.35$

872

$<5.0$

2,410

$<5.0$

$<10$

$<10$

$<10$

$<5.0$

$<10$

$<1.1$

$<5.0$

$<5.0$

$<5.0$

$<5.0$

$<5.0$

6.5

$<1.1$

$<5.0$

$<5.0$

$<5.0$

$<0.11$

$<5.0$

$<100$

$<1.9$

$<2.0$

$<0.054$

452

15

0.79

$<0.54$
Time: $13: 40$

$\mathrm{pH}: 5.3$

Alkalinity: $2 \mathrm{mg} / \mathrm{L}$

Water temperature: $19.2{ }^{\circ} \mathrm{C}$

\begin{tabular}{llll} 
Unit & Flag & Lab \\
\hline $\mathrm{pH}$ & 0 & WA \\
$\mu \mathrm{S} / \mathrm{cm}$ & 0 & WA \\
$\mu \mathrm{g} / \mathrm{L}$ & 0 & WA \\
$\mu \mathrm{g} / \mathrm{L}$ & 0 & WA \\
$\mu \mathrm{g} / \mathrm{L}$ & 0 & WA \\
$\mu \mathrm{g} / \mathrm{L}$ & 0 & WA \\
$\mu \mathrm{g} / \mathrm{L}$ & 0 & WA \\
$\mu \mathrm{g} / \mathrm{L}$ & 0 & WA \\
$\mu \mathrm{g} / \mathrm{L}$ & 0 & WA \\
$\mu \mathrm{g} / \mathrm{L}$ & 0 & WA \\
$\mu \mathrm{g} / \mathrm{L}$ & 0 & WA \\
$\mu \mathrm{g} / \mathrm{L}$ & 0 & WA \\
$\mu \mathrm{g} / \mathrm{L}$ & 0 & WA \\
$\mu \mathrm{g} / \mathrm{L}$ & 0 & WA \\
$\mu \mathrm{g} / \mathrm{L}$ & 0 & WA \\
$\mu \mathrm{g} / \mathrm{L}$ & 0 & WA \\
$\mu \mathrm{g} / \mathrm{L}$ & 0 & WA \\
$\mu \mathrm{g} / \mathrm{L}$ & 0 & WA \\
$\mu \mathrm{g} / \mathrm{L}$ & 0 & WA \\
$\mu \mathrm{g} / \mathrm{L}$ & 0 & WA \\
$\mu \mathrm{g} / \mathrm{L}$ & 0 & WA \\
$\mu \mathrm{g} / \mathrm{L}$ & 0 & WA \\
$\mu \mathrm{g} / \mathrm{L}$ & 0 & WA \\
$\mu \mathrm{g} / \mathrm{L}$ & 0 & WA \\
$\mu \mathrm{g} / \mathrm{L}$ & 0 & WA \\
$\mu \mathrm{g} / \mathrm{L}$ & 0 & WA \\
$\mu \mathrm{g} / \mathrm{L}$ & 0 & WA \\
$\mu \mathrm{g} / \mathrm{L}$ & 0 & WA \\
$\mu \mathrm{g} / \mathrm{L}$ & 0 & WA \\
$\mu \mathrm{g} / \mathrm{L}$ & 0 & WA \\
$\mu \mathrm{g} / \mathrm{L}$ & 0 & WA \\
$\mu \mathrm{g} / \mathrm{L}$ & 0 & WA \\
$\mu \mathrm{g} / \mathrm{L}$ & 0 & WA \\
$\mu \mathrm{g} / \mathrm{L}$ & 0 & WA \\
$\mu \mathrm{g} / \mathrm{L}$ & 0 & W!A \\
$\mu \mathrm{g} / \mathrm{L}$ & 0 & WA \\
$\mu \mathrm{g} / \mathrm{L}$ & 0 & WA \\
$\mu \mathrm{g} / \mathrm{L}$ & 0 & WA \\
$\mu \mathrm{g} / \mathrm{L}$ & 0 & WA \\
& & 0 &
\end{tabular}

- = exceeded riolding time.

- =xceeded primary drinking water standard. 
WELL LFW 48C collected on 06/04/92, laboratory analyses (cont.)

$\begin{array}{ll}\text { H. } & \text { Analyte } \\ \text { Nitrate as nitrogen } \\ \text { Phenols } \\ \text { Potassium } \\ \text { Selenium } \\ \text { Silica } \\ \text { Silver } \\ \text { Sodium } \\ \text { Sulfate } \\ \text { 1,1,2,2-Tetrachloroethane } \\ \text { Tetrachloroethylene } \\ \text { Toluene } \\ \text { Total dissolved solids } \\ \text { Total dissolved solids } \\ \text { Total organic carbon } \\ \text { Total organic halogens } \\ \text { Total phosphates las P) } \\ \text { Toxaphene } \\ \text { 2,4,5-TP (Silvex) } \\ \text { 1,1,1-Trichloroethane } \\ \text { 1,1,2-Trichloroethane } \\ \text { Trichloroethylene } \\ \text { Trichlorofluorornethane } \\ \text { Gross alpha } \\ \text { Nonvolatile beta } \\ \text { Radium-226 } \\ \text { Tritium }\end{array}$

$\begin{aligned} & \text { Result } \\ & 825 \\ &< 5.0 \\ & 618 \\ &< 2.0 \\ & 6,900 \\ & 0.78 \\ & 1.810 \\ & 539 \\ &< 5.0 \\ &< 5.0 \\ &< 5.0 \\ & 14.000 \\ & 15.000 \\ &< 500 \\ & 51 \\ & 41 \\ &< 1.1 \\ &<0.55 \\ &<5.0 \\ &<5.0 \\ &<5.0 \\ &<5.0 \\ &<3.0 \mathrm{E}+00 \\ &<5.0 \mathrm{E}+00 \\ &<5.0 \mathrm{E}-01 \\ & 2.6 \mathrm{E}+00 \pm 3.2 \mathrm{E}-01\end{aligned}$

$\begin{array}{lll}\text { Unit } & \text { Flag } & \text { Lab } \\ \mu \mathrm{g} / \mathrm{L} & 0 & \text { WA } \\ \mu \mathrm{g} / \mathrm{L} & 0 & \text { WA } \\ \mu \mathrm{g} / \mathrm{L} & 0 & \text { WA } \\ \mu \mathrm{g} / \mathrm{L} & 0 & \text { WA } \\ \mu \mathrm{g} / \mathrm{L} & 0 & \text { WA } \\ \mu \mathrm{g} / \mathrm{L} & 0 & \text { WA } \\ \mu \mathrm{g} / \mathrm{L} & 0 & \text { WA } \\ \mu \mathrm{g} / \mathrm{L} & 0 & \text { WA } \\ \mu \mathrm{g} / \mathrm{L} & 0 & \text { WA } \\ \mu \mathrm{g} / \mathrm{L} & 0 & \text { WA } \\ \mu \mathrm{g} / \mathrm{L} & 0 & \text { WA } \\ \mu \mathrm{g} / \mathrm{L} & 0 & \text { WA } \\ \mu \mathrm{g} / \mathrm{L} & 0 & \text { WA } \\ \mu \mathrm{g} / \mathrm{L} & 0 & \text { WA } \\ \mu \mathrm{g} / \mathrm{L} & 2 & \text { WA } \\ \mu \mathrm{g} / \mathrm{L} & 0 & \text { WA } \\ \mu \mathrm{g} / \mathrm{L} & 0 & \text { WA } \\ \mu \mathrm{g} / \mathrm{L} & 0 & \text { WA } \\ \mu \mathrm{g} / \mathrm{L} & 0 & \text { WA } \\ \mu \mathrm{g} / \mathrm{L} & 0 & \text { WA } \\ \mu \mathrm{g} / \mathrm{L} & 0 & \text { WA } \\ \mu \mathrm{g} / \mathrm{L} & 0 & \text { WA } \\ \mathrm{pC \textrm {i } / \mathrm { L }} & 0 & \text { CN } \\ \mathrm{pCi} / \mathrm{L} & 0 & \text { CN } \\ \mathrm{pCi} / \mathrm{L} & 0 & \text { CN } \\ \mathrm{pC \textrm {C } / \mathrm { mL }} & 0 & \text { CN }\end{array}$

\section{WELL. LFW 48D}

$\begin{array}{llllll}\text { SRS Coord. } & \text { Lat/Longitude } & \text { Screen Zone Elevation } & \text { Top of Casing } & \text { Casing } & \text { Formation } \\ \text { N83877.5 } & 33.285424^{\circ} \mathrm{N} & 155.0-134.9 \mathrm{ft} \mathrm{msl} & & 169.5 \mathrm{ft} \mathrm{msl} & 4 \text { " PVC } \\ \text { E45443.7 } & 81.710069^{\circ} \mathrm{W} & & & \end{array}$

\section{MEASUREMENTS CONDUCTED IN THE FIELD}

Sample date: 04/28/92

Depth to water: $20.68 \mathrm{ft}(6.30 \mathrm{~m})$ below TOC Water elevation: $148.82 \mathrm{ft}(45.36 \mathrm{~m}) \mathrm{msl}$

Sp. conductance: $426 \mu \mathrm{S} / \mathrm{cm}$

Water evacuated before sampling: $56 \mathrm{gal}$

\section{LABORATORY ANALYSES}

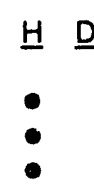

\section{Analyte}

$\mathrm{pH}$

Specific conductance

Specific conductance

Arsenic

Barium

Benzene

Bromodichloromethane

Brornoform

\begin{tabular}{l} 
Result \\
\hline 6.4 \\
277 \\
279 \\
11 \\
5.1 \\
4.3 \\
$<5.0$ \\
$<5.0$
\end{tabular}

Time: $10: 00$

$\mathrm{pH}: 6.9$

Alkalinity: $157 \mathrm{mg} / \mathrm{L}$

Water temperature: $17.6^{\circ} \mathrm{C}$
WA

WA

WA

WA

WA

WA

WA

WA

WA

WA

WA

WA

WA

WA

CN

$\mathrm{CN}$

$\mathrm{CN}$ 
WELL LFW 48D collected on 04/28/92, laboratory analyses (cont.)

H D Analyte

Bromomethane (Methyl bromide)

Cadmium

Calcium

Carbon tetrachloride

Chloride

Chlorobenzene

Chloroethane

- Chloroethene (Vinyl chloride)

2-Chloroethyl vinyl ether

Chloroform

Chloromethane (Methyl chloride)

Chromium

Dibromochloromethane

1,1-Dichloroethane

1,2-Dichloroethane

1,1-Dichloroethylene

trans-1,2-Dichloroethylene

Dichloromethane (Methylene chloride)

2,4-Dichlorophenoxyacetic acid

2,4-Dichlorophenoxyacetic acid

1,2-Dichloropropane

cis-1,3-Dichloropropene

trans-1,3-Dichloropropene

Endrin

Ethylbenzene

Fluoride

Fluoride

Iron

Lead

Lindane

Magnesium

Manganese

Mercury

Mercury

Methoxychlor

Nitrate as nitrogen

Phenols

Phenols

Potassium

Selenium

Silica

Silver

Sodium

Sulfate

$1,1,2,2$-Tetrachloroethane

Tetrachloroethylene

Toluene

Total dissolved solids

Total organic carbon

Total organic carbon

Total organic halogens

Total phosphates (as P)

Total phosphates (as P)

Toxaphene

2,4,5-TP (Siivex)

$2,4,5$-TP (Silvex)
Result

$<10$

$<0.35$

7,880

$<5.0$

25,500

29

$<10$

104

$<10$

$<5.0$

$<10$

$<1.1$

$<5.0$

49

1.9

$<5.0$

$<5.0$

7.2

$<1.1$

$<2.2$

$<5.0$

$<5.0$

$<5.0$

$<0.11$

20

254

254

48,800

$<2.0$

$<0.056$

16,700

18

$<0.20$

$<0.20$

$<0.56$

146

9.5

10

1,400

$<2.0$

5,620

$<0.70$

20,300

336

$<5.0$

$<5.0$

6.0

131,000

14,200

14,000

144

45

39

$<1.1$

$<0.53$

$<1.1$

\begin{tabular}{|c|c|c|}
\hline Unit & Flag & $\underline{\text { Lab }}$ \\
\hline$\mu \mathrm{g} / \mathrm{L}$ & 0 & WA \\
\hline$\mu g / L$ & 0 & WA \\
\hline$\mu g / L$ & 0 & WA \\
\hline$\mu \mathrm{g} / \mathrm{L}$ & 0 & WA \\
\hline$\mu \mathrm{g} / \mathrm{L}$ & 0 & WA \\
\hline$\mu g / L$ & 2 & WA \\
\hline$\mu g / L$ & 0 & WA \\
\hline$\mu \mathrm{g} / \mathrm{L}$ & 2 & WA \\
\hline$\mu \mathrm{g} / \mathrm{L}$ & 0 & WA \\
\hline$\mu \mathrm{g} / \mathrm{L}$ & 0 & WA \\
\hline$\mu \mathrm{g} / \mathrm{L}$ & 0 & WA \\
\hline$\mu g / L$ & 0 & WA \\
\hline$\mu g / L$ & 0 & WA \\
\hline$\mu \mathrm{g} / \mathrm{L}$ & 2 & WA \\
\hline$\mu g / L$ & 0 & WA \\
\hline$\mu g / L$ & 0 & WA \\
\hline$\mu \mathrm{g} / \mathrm{L}$ & 0 & WA \\
\hline$\mu \mathrm{g} / \mathrm{L}$ & 0 & WA \\
\hline$\mu g / L$ & 0 & WA \\
\hline$\mu \mathrm{g} / \mathrm{L}$ & 0 & WA \\
\hline$\mu g / L$ & 0 & WA \\
\hline$\mu \mathrm{g} / \mathrm{L}$ & 0 & WA \\
\hline$\mu \mathrm{g} / \mathrm{L}$ & 0 & WA \\
\hline$\mu \mathrm{g} / \mathrm{L}$ & 0 & WA \\
\hline$\mu g / L$ & 0 & WA \\
\hline$\mu g / L$ & 0 & WA \\
\hline$\mu g / L$ & 0 & WA \\
\hline$\mu \mathrm{g} / \mathrm{L}$ & 2 & WA \\
\hline$\mu g / L$ & 0 & WA \\
\hline$\mu g / L$ & 0 & WA \\
\hline$\mu \mathrm{g} / \mathrm{L}$ & 0 & WA \\
\hline$\mu g / L$ & 0 & WA \\
\hline$\mu g / L$ & 0 & WA \\
\hline$\mu \mathrm{g} / \mathrm{L}$ & 0 & WA \\
\hline$\mu \mathrm{g} / \mathrm{L}$ & 0 & WA \\
\hline$\mu g / L$ & 0 & WA \\
\hline$\mu g / L$ & 0 & WA \\
\hline$\mu \mathrm{g} / \mathrm{L}$ & 0 & WA \\
\hline$\mu \mathrm{g} / \mathrm{L}$ & 0 & WA \\
\hline$\mu \mathrm{g} / \mathrm{L}$ & 0 & WA \\
\hline$\mu \mathrm{g} / \mathrm{L}$ & 0 & WA \\
\hline$\mu g / L$ & 0 & WA \\
\hline$\mu g / L$ & 0 & WA \\
\hline$\mu \mathrm{g} / \mathrm{L}$ & 0 & WA \\
\hline$\mu g / L$ & 0 & WA \\
\hline$\mu g / L$ & 0 & WA \\
\hline$\mu g / L$ & 0 & WA \\
\hline$\mu g / L$ & 0 & WA \\
\hline$\mu g / L$ & 2 & WA \\
\hline$\mu \mathrm{g} / \mathrm{L}$ & 2 & WA \\
\hline$\mu g / L$ & 2 & WA \\
\hline$\mu \mathrm{g} / \mathrm{L}$ & 0 & WA \\
\hline$\mu g / L$ & 0 & WA \\
\hline$\mu \mathrm{g} / \mathrm{L}$ & 0 & WA \\
\hline$\mu g / L$ & 0 & WA \\
\hline$\mu \mathrm{g} / \mathrm{L}$ & 0 & WA \\
\hline
\end{tabular}

- exceeded holding time. = exceeded primary drinking water standard. 
WELL LFW 48D collected on 04/28/92, laboratory analyses (cont.)

$\underline{H}$ D $\quad$ Analyte

1,1,1-Trichloroethane

1,1,2-Trichloroethane

Trichloroethylene

Trichlorofluoromethane

Gross alpha

Nonvolatile beta

Radium-226

Tritium

$\begin{aligned} & \text { Result } \\ &< 5.0 \\ &< 5.0 \\ &<5.0 \\ &<5.0 \\ &<3.0 E+00 \\ &<5.0 E+00 \\ & 9.8 E-01 \pm 9.8 E-02 \\ & 6.2 E+00 \pm 9.7 E-01\end{aligned}$

$\underline{\text { Unit }}$

Flag

$\underline{\text { Lab }}$

$\mu \mathrm{g} / \mathrm{L} \quad 0 \quad$ WA

$\mu g / L \quad 0 \quad$ WA

$\mu g / L \quad 0 \quad$ WA

$\mu g / L \quad 0 \quad$ WA

$\mathrm{pCi} / \mathrm{L} \quad 0 \quad \mathrm{CN}$

$\mathrm{pCi} / \mathrm{L} \quad 0 \quad \mathrm{CN}$

$\mathrm{pCi} / \mathrm{L} \quad 0 \quad \mathrm{CN}$

$\mathrm{pCi} / \mathrm{mL} \quad \mathrm{O} \quad \mathrm{CN}$

\section{WELL LFW 55C}

\begin{tabular}{|c|c|c|c|c|c|}
\hline SRS Coord. & Lat/Longitude & Screen Zone Elevation & Top of Casing & Casing & Formation \\
\hline $\begin{array}{l}\text { N83613.2 } \\
\text { E45205.9 }\end{array}$ & $\begin{array}{l}33.284451{ }^{\circ} \mathrm{N} \\
81.710181{ }^{\circ} \mathrm{W}\end{array}$ & $104.1-94.1 \mathrm{ft} \mathrm{msl}$ & $156.4 \mathrm{ft} \mathrm{msl}$ & 4" PVC & \\
\hline
\end{tabular}

\section{MEASUREMENTS CONDUCTED IN THE FIELD}

Sample date: 06/08/92

Depth to water: $10.08 \mathrm{ft}(3.07 \mathrm{~m})$ ' below TOC

Water elevation: $146.32 \mathrm{ft}(44.60 \mathrm{~m}) \mathrm{msl}$

Sp. conductance: $30 \mu \mathrm{S} / \mathrm{cm}$

Water evacuated before sampling: $138 \mathrm{gal}$

\section{LABORATORY ANALYSES}

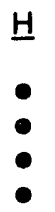

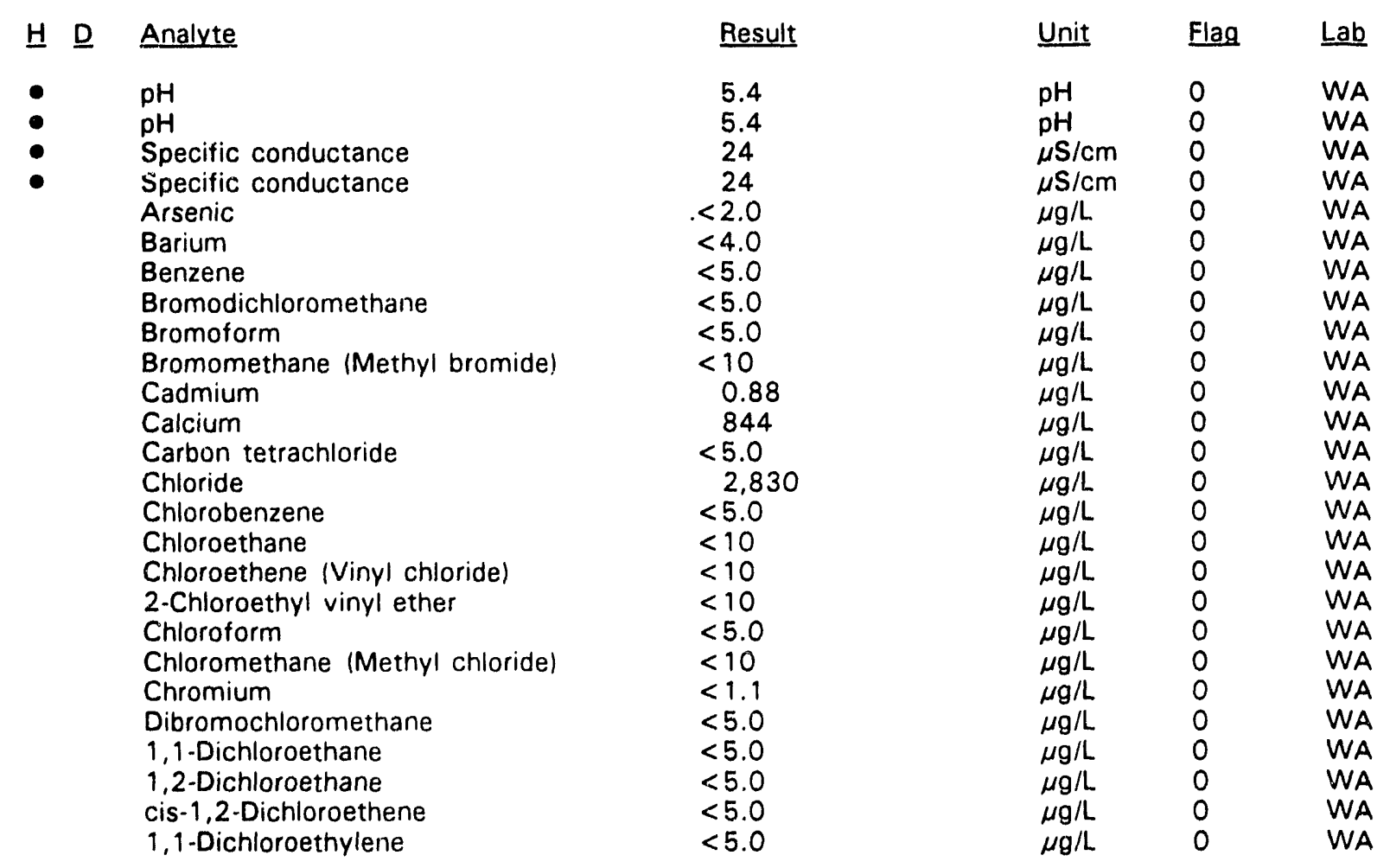

Time: $14: 25$

$\mathrm{pH}: 4.8$

Alkalinity: $1 \mathrm{mg} / \mathrm{L}$

Water temperature: $19.5^{\circ} \mathrm{C}$

$\overline{-=\text { exceeded holding time. }}$ - = exceeded primary drinking water standard. 
WELL LFW 55C collected on 06/08/92, laboratory analyses (cont.)

H D

Dichloromethane (Methylene chloride)

2,4-Dichlorophenoxyacetic acid

2,4-Dichlorophenoxyacetic acid

1,2-Dichloropropane

cis-1,3-Dichloropropene

trans-1,3-Dichloropropene

Endrin

Ethylbenzene

Fluoride

Iron

Lead

Lindane

Magnesium

Manganese

Mercury

Methoxychlor

Nitrate as nitrogen

Phenols

Phenols

Potassium

Selenium

Silica

Silver

Sodium

Sulfate

Sulfate

1,1,2,2-Tetrachloroethane

Tetrachloroethylene

Toluene

Total dissolved solids

Total organic carbon

Total organic halogens

Total phosphates (as P)

Toxaphene

2,4,5-TP (Silvex)

2,4,5-TP (Silvex)

1,1,1-Trichloroethane

1,1,2-Trichloroethane

Trichloroethylene

Trichlorofluoromethane

Gross alpha

Nonvolatile beta

Radium-226

Tritium

\section{Result}

13

$<1.1$

$<2.2$

$<5.0$

$<5.0$

$<5.0$

$<0.11$

$<5.0$

$<100$

$<1.9$

$<2.0$

$<0.056$

934

5.6

$<0.20$

$<0.56$

1,280

$<5.0$

$<5.0$

539

$<2.0$

7,510

$<0.70$

1.950

551

554

$<5.0$

$<5.0$

$<5.0$

20,000

$<500$

27

22

$<1.1$

$<0.55$

$<1.1$

$<5.0$

$<5.0$

$<5.0$

$<5.0$

$<3.0 E+00$

$<5.0 E+00$

$<5.0$ E-0 1

$<2.0 E+00$

\begin{tabular}{|c|c|c|}
\hline Unit & Flag & $\underline{L a b}$ \\
\hline$\mu g / L$ & 0 & WA \\
\hline$\mu \mathrm{g} / \mathrm{L}$ & 0 & WA \\
\hline$\mu \mathrm{g} / \mathrm{L}$ & 0 & WA \\
\hline$\mu g / L$ & 0 & WA \\
\hline$\mu \mathrm{g} / \mathrm{L}$ & 0 & WA \\
\hline$\mu g / L$ & 0 & WA \\
\hline$\mu g / L$ & 0 & WA \\
\hline$\mu \mathrm{g} / \mathrm{L}$ & 0 & WA \\
\hline$\mu \mathrm{g} / \mathrm{L}$ & 0 & WA \\
\hline$\mu g / L$ & 0 & WA \\
\hline$\mu \mathrm{g} / \mathrm{L}$ & 0 & WA \\
\hline$\mu \mathrm{g} / \mathrm{L}$ & 0 & WA \\
\hline$\mu \mathrm{g} / \mathrm{L}$ & 0 & WA \\
\hline$\mu \mathrm{g} / \mathrm{L}$ & 0 & WA \\
\hline$\mu g / L$ & 0 & WA \\
\hline$\mu \mathrm{g} / \mathrm{L}$ & 0 & WA \\
\hline$\mu \mathrm{g} / \mathrm{L}$ & 0 & WA \\
\hline$\mu \mathrm{g} / \mathrm{L}$ & 0 & WA \\
\hline$\mu g / L$ & 0 & WA \\
\hline$\mu \mathrm{g} / \mathrm{L}$ & 0 & WA \\
\hline$\mu \mathrm{g} / \mathrm{L}$ & 0 & WA \\
\hline$\mu \mathrm{g} / \mathrm{L}$ & 0 & WA \\
\hline$\mu \mathrm{g} / \mathrm{L}$ & 0 & WA \\
\hline$\mu \mathrm{g} / \mathrm{L}$ & 0 & WA \\
\hline$\mu \mathrm{g} / \mathrm{L}$ & 0 & WA \\
\hline$\mu g / L$ & 0 & WA \\
\hline$\mu \mathrm{g} / \mathrm{L}$ & 0 & WA \\
\hline$\mu \mathrm{g} / \mathrm{L}$ & 0 & WA \\
\hline$\mu \mathrm{g} / \mathrm{L}$ & 0 & WA \\
\hline$\mu \mathrm{g} / \mathrm{L}$ & 0 & WA \\
\hline$\mu \mathrm{g} / \mathrm{L}$ & 0 & WA \\
\hline$\mu \mathrm{g} / \mathrm{L}$ & 1 & WA \\
\hline$\mu g / L$ & 0 & WA \\
\hline$\mu \mathrm{g} / \mathrm{L}$ & 0 & WA \\
\hline$\mu \mathrm{g} / \mathrm{L}$ & 0 & WA \\
\hline$\mu g / L$ & 0 & WA \\
\hline$\mu \mathrm{g} / \mathrm{L}$ & 0 & WA \\
\hline$\mu \mathrm{g} / \mathrm{L}$ & 0 & WA \\
\hline$\mu g / L$ & 0 & WA \\
\hline$\mu \mathrm{g} / \mathrm{L}$ & 0 & WA \\
\hline $\mathrm{pCi} / \mathrm{L}$ & 0 & $\mathrm{CN}$ \\
\hline $\mathrm{pCi} / \mathrm{L}$ & 0 & $\mathrm{CN}$ \\
\hline $\mathrm{pCi} / \mathrm{L}$ & 0 & $\mathrm{CN}$ \\
\hline $\mathrm{pCi} / \mathrm{mL}$ & 0 & $\mathrm{CN}$ \\
\hline
\end{tabular}

C

- = exceeded holding time. $=$ exceeded primary drinking water standard. 
WELL LFW 55D

\begin{tabular}{|c|c|c|c|c|}
\hline SRS Coord. & Lat/Longitude & Screen Zone Elevation & Top of Casing & $\underline{\text { Casing }}$ \\
\hline $\begin{array}{l}\text { N83601.3 } \\
\text { E45189.3 }\end{array}$ & $\begin{array}{l}33.284398^{\circ} \mathrm{N} \\
81.710202^{\circ} \mathrm{W}\end{array}$ & $141.4-121.2 \mathrm{ft} \mathrm{msl}$ & $\mathrm{msl}$ & PVC \\
\hline
\end{tabular}

MEASUREMENTS CONDUCTED IN THE FIELD

Sample date: 06/08/92

Dep th to water: $9.05 \mathrm{ft}(2.76 \mathrm{~m})$ below TOC

Water elevation: $146.35 \mathrm{ft}(44.61 \mathrm{~m}) \mathrm{msl}$

Sp. conductance: $29 \mu \mathrm{S} / \mathrm{cm}$

Water evacuated before sampling: 66 gal

Time: $14: 05$

pH: 4.6

Alkalinity: $1 \mathrm{mg} / \mathrm{L}$

Water temperature: $18.3^{\circ} \mathrm{C}$

\section{LABORATORY ANALYSES}

H D Analyte

\begin{tabular}{|c|c|}
\hline 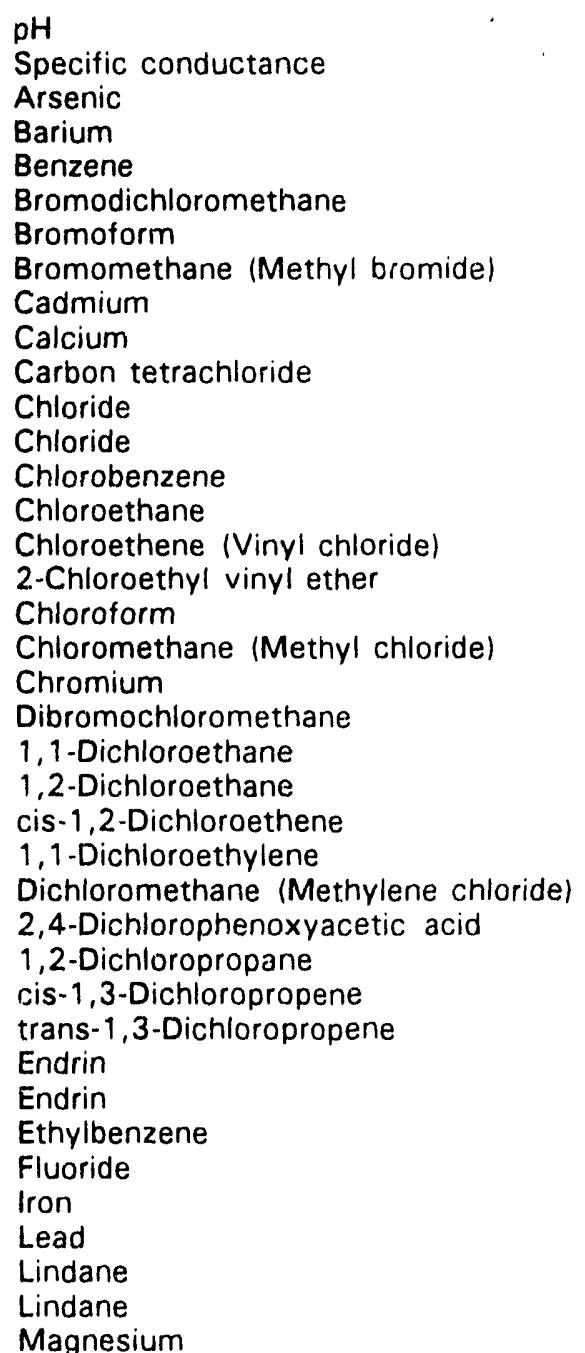 & $\begin{aligned} & 5.4 \\
& 25 \\
&< 2.0 \\
& 8.6 \\
&< 5.0 \\
&< 5.0 \\
&< 5.0 \\
&< 10 \\
& 0.75 \\
& 1.150 \\
&< 5.0 \\
& 2.710 \\
& 2.740 \\
&< 5.0 \\
&< 10 \\
&< 10 \\
&< 10 \\
&< 5.0 \\
&< 10 \\
&< 1.1 \\
&< 5.0 \\
&<5.0 \\
&<5.0 \\
&<5.0 \\
&<5.0 \\
& 9.4 \\
&< 1.1 \\
&<5.0 \\
&<5.0 \\
&<5.0 \\
&<0.11 \\
&<0.43 \\
&<5.0 \\
&<100 \\
&<1.9 \\
&<2.0 \\
&<0.054 \\
&<0.22 \\
& 938\end{aligned}$ \\
\hline
\end{tabular}

\begin{tabular}{|c|c|c|}
\hline Urit & Flag & $\underline{L a b}$ \\
\hline $\mathrm{pH}$ & 0 & WA \\
\hline$\mu \mathrm{S} / \mathrm{cm}$ & 0 & WA \\
\hline$\mu \mathrm{g} / \mathrm{L}$ & 0 & WA \\
\hline$\mu g / L$ & 0 & WA \\
\hline$\mu \mathrm{g} / \mathrm{L}$ & 0 & WA \\
\hline$\mu \mathrm{g} / \mathrm{L}$ & 0 & WA \\
\hline$\mu \mathrm{g} / \mathrm{L}$ & 0 & WA \\
\hline$\mu \mathrm{g} / \mathrm{L}$ & 0 & WA \\
\hline$\mu \mathrm{g} / \mathrm{L}$ & 0 & WA \\
\hline$\mu \mathrm{g} / \mathrm{L}$ & 0 & WA \\
\hline$\mu \mathrm{g} / \mathrm{L}$ & 0 & WA \\
\hline$\mu g / L$ & 0 & WA \\
\hline$\mu \mathrm{g} / \mathrm{L}$ & 0 & WA \\
\hline$\mu \mathrm{g} / \mathrm{L}$ & 0 & WA \\
\hline$\mu \mathrm{g} / \mathrm{L}$ & 0 & WA \\
\hline$\mu \mathrm{g} / \mathrm{L}$ & 0 & WA \\
\hline$\mu \mathrm{g} / \mathrm{L}$ & 0 & WA \\
\hline$\mu \mathrm{g} / \mathrm{L}$ & 0 & WA \\
\hline$\mu \mathrm{g} / \mathrm{L}$ & 0 & WA \\
\hline$\mu g / L$ & 0 & WA \\
\hline$\mu \mathrm{g} / \mathrm{L}$ & 0 & WA \\
\hline$\mu \mathrm{g} / \mathrm{L}$ & 0 & WA \\
\hline$\mu \mathrm{g} / \mathrm{L}$ & 0 & WA \\
\hline$\mu \mathrm{g} / \mathrm{L}$ & 0 & WA \\
\hline$\mu \mathrm{g} / \mathrm{L}$ & 0 & WA \\
\hline$\mu \mathrm{g} / \mathrm{L}$ & 0 & WA \\
\hline$\mu \mathrm{g} / \mathrm{L}$ & 0 & WA \\
\hline$\mu \mathrm{g} / \mathrm{L}$ & 0 & WA \\
\hline$\mu \mathrm{g} / \mathrm{L}$ & 0 & WA \\
\hline$\mu \mathrm{g} / \mathrm{L}$ & 0 & WA \\
\hline$\mu \mathrm{g} / \mathrm{L}$ & 0 & WA \\
\hline$\mu \mathrm{g} / \mathrm{L}$ & 0 & WA \\
\hline$\mu \mathrm{g} / \mathrm{L}$ & 0 & WA \\
\hline$\mu \mathrm{g} / \mathrm{L}$ & 0 & WA \\
\hline$\mu \mathrm{J} / \mathrm{L}$ & 0 & WA \\
\hline$\mu \mathrm{g} / \mathrm{L}$ & 0 & WA \\
\hline$\mu g / L$ & 0 & WA \\
\hline$\mu \mathrm{g} / \mathrm{L}$ & 0 & WA \\
\hline & 0 & $W$ \\
\hline
\end{tabular}

$\overline{0=\text { exceeded holding time. }} \boldsymbol{\square}=$ exceeded primary drinking water standard. 
WELL LFW 55D collected on 06/08/92, laboratory analyses (cont.)

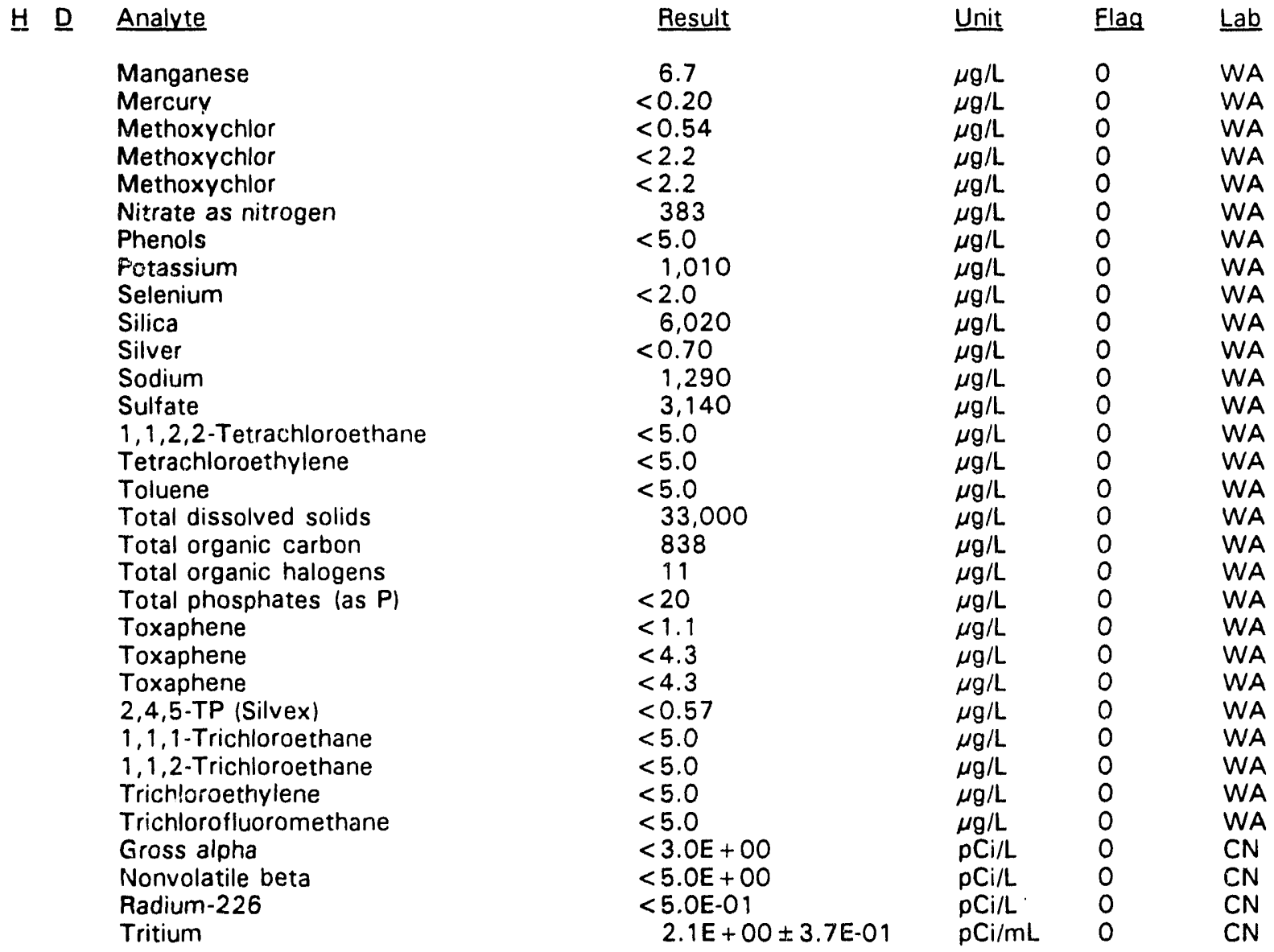

- = exceeded holding time. = exceeded primary drinking water standard. 
WELL LFW 56D

\begin{tabular}{|c|c|c|c|c|}
\hline SRS Coord. & Lat/Longitude & Screen Zone Elevation & Top of Casing & Casing \\
\hline $\begin{array}{l}\text { N83398.0 } \\
\text { E45306.6 }\end{array}$ & $\begin{array}{l}33.284140^{\circ} \mathrm{N} \\
81.709498^{\circ} \mathrm{W}\end{array}$ & $151.4-131.3 \mathrm{ft} \mathrm{msl}$ & $158.1 \mathrm{ft} \mathrm{msl}$ & 4" PVC \\
\hline
\end{tabular}

MEASUREMENTS CONDUCTED IN THE FIELD

Sample date: 06/08/92

Depth to water: $13.20 \mathrm{ft}(4.02 \mathrm{~m})$ below TOC

Water elevation: $144.90 \mathrm{ft}(44.17 \mathrm{~m} / \mathrm{msl}$

Sp. conductance: $23 \mu \mathrm{S} / \mathrm{cm}$

Water evacuated before sampling: $36 \mathrm{gal}$

Time: $14: 55$

$\mathrm{pH}: 4.3$

Alkalinity: $0 \mathrm{mg} / \mathrm{L}$

Water temperature: $18.7^{\circ} \mathrm{C}$

\section{LABORATORY ANALYSES}

\begin{tabular}{|c|c|c|c|c|c|}
\hline$\underline{H} \underline{D}$ & Analyte & Result & $\underline{\text { Unit }}$ & Flag & $\underline{\text { Lab }}$ \\
\hline$\bullet$ & $\mathrm{pH}$ & 5.3 & $\mathrm{pH}$ & 0 & WA \\
\hline & $\begin{array}{l}\text { Specific conductance } \\
\text { Arsenic }\end{array}$ & & $\mu \mathrm{S} / \mathrm{cm}$ & $\begin{array}{l}0 \\
0\end{array}$ & $\begin{array}{l}\text { WA } \\
\text { WA }\end{array}$ \\
\hline & $\begin{array}{l}\text { Arsenic } \\
\text { Barium }\end{array}$ & $\begin{array}{r}<2.0 \\
4.3\end{array}$ & $\begin{array}{l}\mu \mathrm{g} / \mathrm{L} \\
\mu \mathrm{g} / \mathrm{L}\end{array}$ & $\begin{array}{l}0 \\
0\end{array}$ & $\begin{array}{l}\text { WA } \\
\text { WA }\end{array}$ \\
\hline & Benzene & $<5.0$ & $\mu \mathrm{g} / \mathrm{L}$ & 0 & WA \\
\hline & Bromodichloromethane & $<5.0$ & $\mu \mathrm{g} / \mathrm{L}$ & 0 & WA \\
\hline & Bromoform & $<5.0$ & $\mu \mathrm{g} / \mathrm{L}$ & 0 & WA \\
\hline & Bromomethane (Methyl bromide) & $<10$ & $\mu \mathrm{g} / \mathrm{L}$ & 0 & WA \\
\hline & Cadmium & $<0.35$ & $\mu \mathrm{g} / \mathrm{L}$ & 0 & WA \\
\hline & Calcium & 479 & $\mu \mathrm{g} / \mathrm{L}$ & 0 & \\
\hline & Carbon tetrachloride & $<5.0$ & $\mu \mathrm{g} / \mathrm{L}$ & 0 & WA \\
\hline & Chloride & 3,570 & $\mu g / L$ & 0 & WA \\
\hline & Chlorobenzene & $<5.0$ & $\mu \mathrm{g} / \mathrm{L}$ & 0 & \\
\hline & Chloroethane & $<10$ & $\mu \mathrm{g} / \mathrm{L}$ & 0 & WA \\
\hline & Chloroethene (Vinyl chloride) & $<10$ & $\mu \mathrm{g} / \mathrm{L}$ & 0 & WA \\
\hline & 2-Chloroethyl vinyl ether & $<10$ & $\mu \mathrm{g} / \mathrm{L}$ & 0 & WA \\
\hline & Chloroform & $<5.0$ & $\mu \mathrm{g} / \mathrm{L}$ & 0 & WA \\
\hline & Chloromethane (Methyl chloride) & $<10$ & $\mu \mathrm{g} / \mathrm{L}$ & 0 & WA \\
\hline & Chromiım & $<4.1$ & $\mu \mathrm{g} / \mathrm{L}$ & 0 & WA \\
\hline & Dibromochloromethane & $<5.0$ & $\mu \mathrm{g} / \mathrm{L}$ & 0 & WA \\
\hline & 1,1-Dichloroethane & 1.7 & $\mu \mathrm{g} / \mathrm{L}$ & 0 & WA \\
\hline & 1,2-Dichloroethane & $<5.0$ & $\mu \mathrm{g} / \mathrm{L}$ & 0 & WA \\
\hline & cis-1,2-Dichloroethene & $<5.0$ & $\mu \mathrm{g} / \mathrm{L}$ & 0 & WA \\
\hline & 1.1-Dichloroethylene & $<5.0$ & $\mu \mathrm{g} / \mathrm{L}$ & 0 & WA \\
\hline & Dichloromethane (Methylene chloride) & 6.2 & $\mu \mathrm{g} / \mathrm{L}$ & 0 & WA \\
\hline & 2,4-Dichlorophenoxyacetic acid & $<1.1$ & $\mu \mathrm{g} / \mathrm{L}$ & 0 & WA \\
\hline & 1,2-Dichloropropane & $<5.0$ & $\mu \mathrm{g} / \mathrm{L}$ & 0 & WA \\
\hline & cis-1,3-Dichloropropene & $<5.0$ & $\mu \mathrm{g} / \mathrm{L}$ & 0 & WA \\
\hline & trans-1,3-Dichloropropene & $<5.0$ & $\mu \mathrm{g} / \mathrm{L}$ & 0 & WA \\
\hline & Endrin & $<0.11$ & $\mu \mathrm{g} / \mathrm{L}$ & 0 & WA \\
\hline & Ethylbenzene & $<5.0$ & $\mu \mathrm{g} / \mathrm{L}$ & 0 & WA \\
\hline & Fluoride & $<100$ & $\mu \mathrm{g} / \mathrm{L}$ & 0 & WA \\
\hline & Iron & 2.1 & $\mu \mathrm{g} / \mathrm{L}$ & 0 & WA \\
\hline & Lead & $<2.0$ & $\mu \mathrm{g} / \mathrm{L}$ & 0 & w \\
\hline & Lindane & $<0.054$ & $\mu \mathrm{g} / \mathrm{L}$ & 0 & WA \\
\hline & Magnesium & 4.38 & $\mu \mathrm{g} / \mathrm{L}$ & 0 & WA \\
\hline & Manganese & 1.8 & $\mu \mathrm{g} / \mathrm{L}$ & 0 & WA \\
\hline & Mercury & $<0.20$ & $\mu \mathrm{g} / \mathrm{L}$ & 0 & WA \\
\hline & Mercury & $<0.20$ & $\mu \mathrm{g} / \mathrm{L}$ & 0 & A \\
\hline
\end{tabular}

- = exceeded holding time. $=$ exceeded primary drinking water standard. 
WELL LFW 56D collected on 06/08/92, laboratory analyses (cont.)

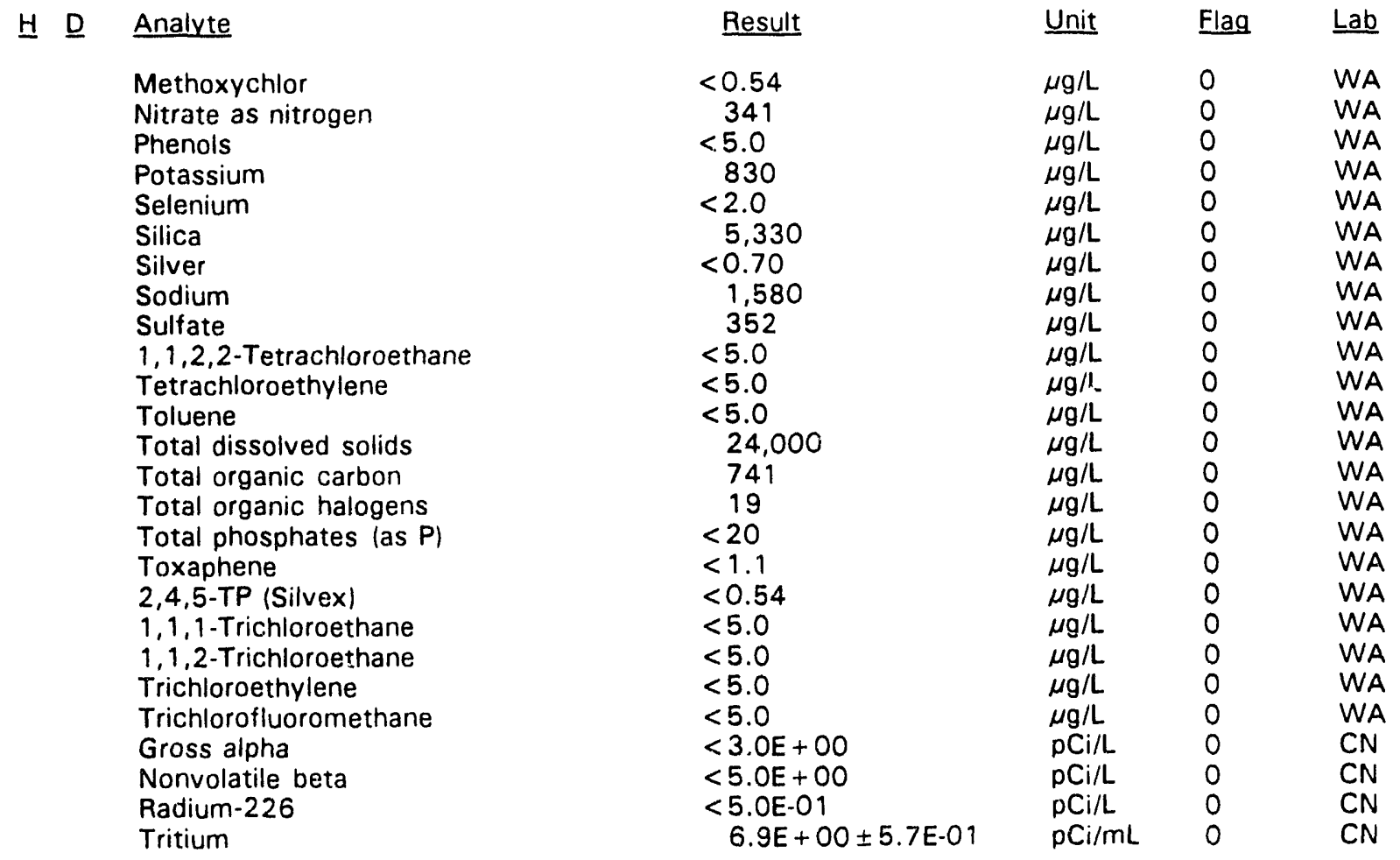

\section{WELL LFW 57B}

$\begin{array}{llllll}\text { SRS Coord. } & \text { Lat/Longitude } & \text { Screen Zone Elevation } & \text { Top of Casing } & \text { Casing } & \text { Formation } \\ \text { N83196.7 } & 33.283914^{\circ} \mathrm{N} & 78.4-58.4 \mathrm{ft} \mathrm{msl} & 165.4 \mathrm{ft} \mathrm{msl} & \text { 4" PVC } \\ \text { E45440.6 } & 81.708754^{\circ} \mathrm{W} & & & \end{array}$

\section{MEASUREMENTS CONDUCTED IN THE FIELD}

Sample date: 06/08/92

Depth to water: $22.14 \mathrm{ft}(6.75 \mathrm{~m})$ below TOC Water elevation: $143.26 \mathrm{ft}(43.67 \mathrm{~m}) \mathrm{msl}$

Sp. conductance: $65 \mu \mathrm{S} / \mathrm{cm}$

Water evacuated before sampling: $196 \mathrm{gal}$

\section{LABORATORY ANALYSES}

\section{H D Analyte}

$\mathrm{pH}$

Specific conductance

Arsenic

Barium

Benzene

Bromodichloromethane

Bromoform

Bromomethane (Methyl bromide)
Result

$$
4.3
$$$$
52
$$$$
<2.0
$$

$<5.0$

$<5.0$

$<5.0$

$<10$
Time: $13: 30$

pH: 3.7

Alkalinity: $0 \mathrm{mg} / \mathrm{L}$

Water temperature: $19.5^{\circ} \mathrm{C}$

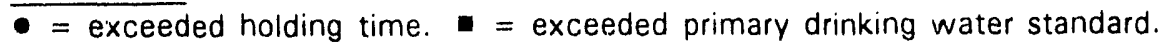

$\begin{array}{lll}\text { Unit } & \text { Flag } & \text { Lab } \\ \mathrm{pH} & 0 & \text { WA } \\ \mu \mathrm{S} / \mathrm{cm} & 0 & \text { WA } \\ \mu \mathrm{g} / \mathrm{L} & 0 & \text { WA } \\ \mu \mathrm{g} / \mathrm{L} & 0 & \text { WA } \\ \mu \mathrm{g} / \mathrm{L} & 0 & \text { WA } \\ \mu \mathrm{g} / \mathrm{L} & 0 & \text { WA } \\ \mu \mathrm{g} / \mathrm{L} & 0 & \text { WA } \\ \mu \mathrm{g} / \mathrm{L} & 0 & \text { WA }\end{array}$


WELL LFW 57B collected on 06/08/92, laboratory analyses (cont.)

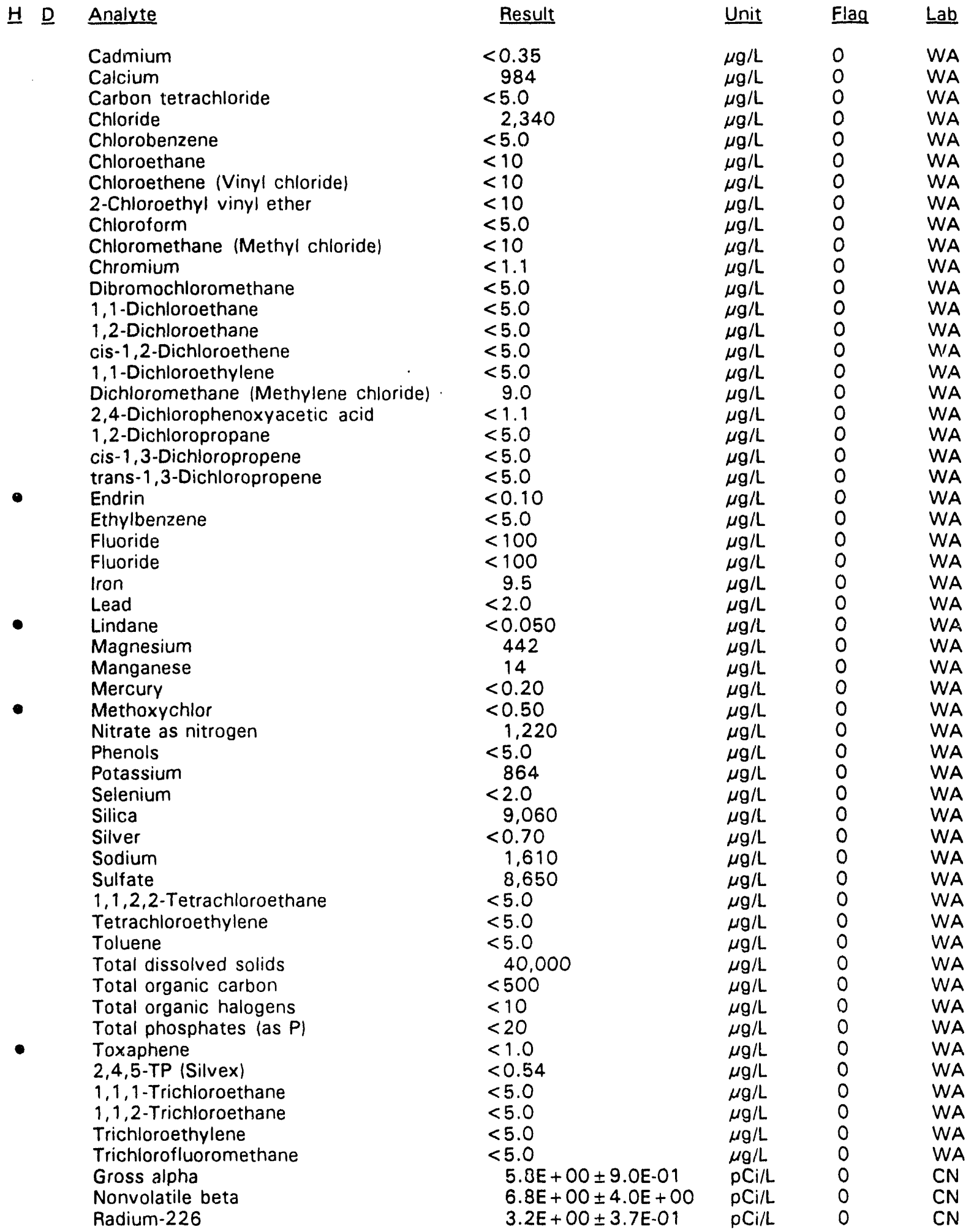

$\overline{-=\text { exceeded holding time. }}$ = exceeded primary drinking water standard. 
WELL LFW 57B collected on 06/08/92, laboratory analyses (cont.)

브 $\underline{\text { Analyte }} \begin{array}{lllll}\text { Tritium } & \underline{\text { Result }} & \text { Unit } & \underline{\text { Flag }} & \underline{\mathrm{Lab}} \\ & <2.0 \mathrm{E}+00 & \mathrm{pCi} / \mathrm{mL} & 0 & \mathrm{CN}\end{array}$

\section{WELL LFW 57C}

$\begin{array}{llllll}\text { SRS Coord. } & \text { Lat/Longitude } & \text { Screen Zone Elevation } & \text { Top of Casing } & \text { Casing } & \text { Formation } \\ \text { N83200.1 } & 33.283873^{\circ} ! ! & 117.9-107.8 \mathrm{ft} \mathrm{msl} & 165.0 \mathrm{ft} \mathrm{msi} & \text { 4" PVC } \\ \text { E45411.1 } & 81.708838^{\circ} \mathrm{W} & & & \end{array}$

MEASUREMENTS CONDUCTED IN THE FIELD

Sample date: 06/08/92

Depth to water: $21.66 \mathrm{ft}(6.60 \mathrm{~m})$ below TOC Water elevation: $143.34 \mathrm{ft}(43.69 \mathrm{~m}) \mathrm{msl}$ Sp. conductance: $31 \mu \mathrm{S} / \mathrm{cm}$

Water evacuated before sampling: $93 \mathrm{gal}$

\section{LABORATORY ANALYSES}

H D Analyte

- $\quad \mathrm{pH}$

SH

Arsenic

Barium

Benzene

Bromodichloromethane

Bromoform

Bromomethane (Methyl bromide)

Cadmium

Calcium

Carbon tetrachloride

Chloride

Chlorobenzene

Chloroethane

Chloroethene (Vinyl chloride)

2-Chloroethyl vinyl ether

Chloroform

Chloromethane (Methyl chloride)

Chromium

Dibromochloromethane

1,1-Dichloroethane

1,2-Dichloroethane

cis-1,2-Dichloroethene

1,1-Dichloroethylene

Dichloromethane (Methylene chloride)

2.4-Dichlorophenoxyacetic acid

1,2-Dichloropropane

cis-1,3-Dich oropropene

trans-1,3-Dichloropropene

Endrin

Ethylbenzene

Fluoride

Iron
Time: 13:00

$\mathrm{pH}: 4.8$

Alkalinity: $0 \mathrm{mg} / \mathrm{L}$

Water temperature: $19.6^{\circ} \mathrm{C}$

$\begin{array}{llll}\text { Result } & \text { Unit } & \text { Flag } & \text { Lab } \\ 5.2 & \mu H & 0 & \text { WA } \\ 25 & \mu \mathrm{S} / \mathrm{cm} & 0 & \text { WA } \\ <2.0 & \mu \mathrm{g} / \mathrm{L} & 0 & \text { WA } \\ 5.8 & \mu \mathrm{g} / \mathrm{L} & 0 & \text { WA } \\ <5.0 & \mu \mathrm{g} / \mathrm{L} & 0 & \text { WA } \\ <5.0 & \mu \mathrm{g} / \mathrm{L} & 0 & \text { WA } \\ <5.0 & \mu \mathrm{g} / \mathrm{L} & 0 & \text { WA } \\ <10 & \mu \mathrm{g} / \mathrm{L} & 0 & \text { WA } \\ 0.75 & \mu \mathrm{g} / \mathrm{L} & 0 & \text { WA } \\ 1.210 & \mu \mathrm{g} / \mathrm{L} & 0 & \text { WA } \\ <5.0 & \mu \mathrm{g} / \mathrm{L} & 0 & \text { WA } \\ 2.570 & \mu \mathrm{g} / \mathrm{L} & 0 & \text { WA } \\ <5.0 & \mu \mathrm{g} / \mathrm{L} & 0 & \text { WA } \\ <10 & \mu \mathrm{g} / \mathrm{L} & 0 & \text { WA } \\ <10 & \mu \mathrm{g} / \mathrm{L} & 0 & \text { WA } \\ <10 & \mu \mathrm{g} / \mathrm{L} & 0 & \text { WA } \\ <5.0 & \mu \mathrm{g} / \mathrm{L} & 0 & \text { WA } \\ <10 & \mu \mathrm{g} / \mathrm{L} & 0 & \text { WA } \\ <1.1 & \mu \mathrm{g} / \mathrm{L} & 0 & \text { WA } \\ <5.0 & \mu \mathrm{g} / \mathrm{L} & 0 & \text { WA } \\ <5.0 & \mu \mathrm{g} / \mathrm{L} & 0 & \text { WA } \\ <5.0 & \mu \mathrm{g} / \mathrm{L} & 0 & \text { WA } \\ <5.0 & \mu \mathrm{g} / \mathrm{L} & 0 & \text { WA } \\ <5.0 & \mu \mathrm{g} / \mathrm{L} & 0 & \text { WA } \\ 12 & \mu \mathrm{g} / \mathrm{L} & 0 & \text { WA } \\ <1.1 & \mu \mathrm{g} / \mathrm{L} & 0 & \text { WA } \\ <5.0 & \mu \mathrm{g} / \mathrm{L} & 0 & \text { WA } \\ <5.0 & \mu \mathrm{g} / \mathrm{L} & 0 & \text { WA } \\ <5.0 & \mu \mathrm{g} / \mathrm{L} & 0 & \text { WA } \\ <0.11 & \mu \mathrm{g} / \mathrm{L} & 0 & \text { WA } \\ <5.0 & \mu \mathrm{g} / \mathrm{L} & 0 & \text { WA } \\ <100 & \mu \mathrm{g} / \mathrm{L} & 0 & \text { WA } \\ <1.9 & \mu \mathrm{g} / \mathrm{L} & 0 & \text { WA } \\ & & & \\ & & 0 & 0\end{array}$

- exceeded holding time.

= exceeded primary drinking water standard. 
WELL LFW 57C collected on 06/08/92, laboratory analyses (cont.)

H $\underline{D}$ Analyte

Lead
Lindane
Magnesium
Manganese
Mercury
Methoxychlor
Nitrate as nitrogen
Phenols
Potassium
Selenium
Silica
Silver
Sodium
Sulfate
1,1,2,2-Tetrachloroethane
Tetrachloroethylene
Toluene
Total dissolved solids
Total organic carbon
Total organic halogens
Total phosphates (as P)
Toxaphene
2,4,5-TP (Silvex)
$1,1,1-$ Trichloroethane
1,1,2-Trichloroethane
Trichloroethylene
Trichlorofluoromethane
Gross alpha
Nonvolatile beta
Radium-226
Tritium

$\begin{array}{rl} & \text { Result } \\ < & 2.0 \\ < & 0.054 \\ & 915 \\ & 5.8 \\ < & 0.20 \\ < & 0.54 \\ & 1.420 \\ < & 5.0 \\ & 619 \\ < & 2.0 \\ & 7.800 \\ < & 0.70 \\ & 1.900 \\ & 650 \\ <5.0 \\ <5.0 \\ <5.0 \\ & 35.000 \\ <500 & 18 \\ & 23 \\ <1.1 \\ <0.55 \\ <5.0 \\ <5.0 \\ <5.0 \\ <5.0 \\ <3.0 E+00 \\ <5.0 E+00 \\ & 7.0 \mathrm{E}-01 \pm 1.9 \mathrm{E}-01 \\ <2.0 \mathrm{E}+00 \\ \end{array}$

\begin{tabular}{|c|c|c|}
\hline Unit & Flag & Lab \\
\hline$\mu \mathrm{g} / \mathrm{L}$ & 0 & WA \\
\hline$\mu \mathrm{g} / \mathrm{L}$ & 0 & WA \\
\hline$\mu \mathrm{g} / \mathrm{L}$ & 0 & WA \\
\hline$\mu \mathrm{g} / \mathrm{L}$ & 0 & WA \\
\hline$\mu \mathrm{g} / \mathrm{L}$ & 0 & WA \\
\hline$\mu g / L$ & 0 & WA \\
\hline$\mu \mathrm{g} / \mathrm{L}$ & 0 & WA \\
\hline$\mu g / L$ & 0 & WA \\
\hline$\mu \mathrm{g} / \mathrm{L}$ & 0 & WA \\
\hline$\mu \mathrm{g} / \mathrm{L}$ & 0 & WA \\
\hline$\mu \mathrm{g} / \mathrm{L}$ & 0 & WA \\
\hline$\mu \mathrm{g} / \mathrm{L}$ & 0 & WA \\
\hline$\mu \mathrm{g} / \mathrm{L}$ & 0 & WA \\
\hline$\mu \mathrm{g} / \mathrm{L}$ & 0 & WA \\
\hline$\mu \mathrm{g} / \mathrm{L}$ & 0 & WA \\
\hline$\mu g / L$ & 0 & WA \\
\hline$\mu \mathrm{g} / \mathrm{L}$ & 0 & WA \\
\hline$\mu \mathrm{g} / \mathrm{L}$ & 0 & WA \\
\hline$\mu g / L$ & 0 & WA \\
\hline$\mu \mathrm{g} / \mathrm{L}$ & 0 & WA \\
\hline$\mu \mathrm{g} / \mathrm{L}$ & 0 & WA \\
\hline$\mu \mathrm{g} / \mathrm{L}$ & 0 & WA \\
\hline$\mu \mathrm{g} / \mathrm{L}$ & 0 & WA \\
\hline$\mu g / L$ & 0 & WA \\
\hline$\mu g / L$ & 0 & WA \\
\hline$\mu \mathrm{g} / \mathrm{L}$ & 0 & WA \\
\hline$\mu \mathrm{g} / \mathrm{L}$ & 0 & WA \\
\hline $\mathrm{pCi} / \mathrm{L}$ & 0 & $\mathrm{CN}$ \\
\hline $\mathrm{pCi} / \mathrm{L}$ & 0 & $\mathrm{CN}$ \\
\hline $\mathrm{pCi} / \mathrm{L}$ & 0 & $\mathrm{CN}$ \\
\hline $\mathrm{pCi} / \mathrm{mL}$ & 0 & $\mathrm{CN}$ \\
\hline
\end{tabular}

\section{WELL LFW 57D}

\begin{tabular}{|c|c|c|c|c|c|}
\hline SRS Coord. & Lat/Longitude & Screen Zone Elevation & Top nf Casing & Casing & Formation \\
\hline $\begin{array}{l}\text { N83190.2 } \\
E 45417.4\end{array}$ & $\begin{array}{l}33.283861^{\circ} \mathrm{N} \\
81.708802^{\circ} \mathrm{W}\end{array}$ & $150.4-130.6 \mathrm{ft} \mathrm{msl}$ & $164.8 \mathrm{ft} \mathrm{msl}$ & 4" PVC & \\
\hline
\end{tabular}

\section{MEASUREMENTS CONDUCTED IN THE FIELD}

Sample date: 06/08/92

Depth to water: $21.45 \mathrm{ft}(6.54 \mathrm{~m})$ below TOC Water elevation: $143.35 \mathrm{ft}(43.69 \mathrm{~m}) \mathrm{msl}$ Sp. conductance: $117 \mu \mathrm{S} / \mathrm{cm}$

Water evacuated before sampling: 41 gal

\section{LABORATORY ANALYSES}

Time: $12: 40$

$\mathrm{pH}: 5.5$

Alkalinity: $16 \mathrm{mg} / \mathrm{L}$

Water temperature: $18.1^{\circ} \mathrm{C}$

$\begin{array}{llllll}\text { H } & \text { Analyte } & \text { Result } & \text { Unit } & \underline{\text { Flag }} & \underline{\text { Lab }} \\ \text { - } & \mathrm{pH} & 6.1 & \mathrm{pH} & 0 & \text { WA } \\ \text { - } & \text { Specific conductance } & 139 & \mu \mathrm{S} / \mathrm{cm} & 0 & \text { WA } \\ \text { Arsenic } & <2.0 & \mu \mathrm{g} / \mathrm{L} & 0 & \text { WA }\end{array}$

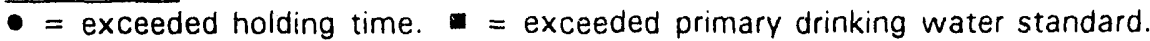

Sanitary Landfill 
WELL LFW 57D collected on 06/08/92, laboratory analyses (cont.)

H D Analyte

Barium

Benzene

Bromodichloromethane

Bromoform

Bromomethane (Methyl bromide)

Cadmium

Calcium

Carbon tetrachloride

Chloride

Chlorobenzene

Chloroethane

Chloroethene (Vinyl chloride)

2-Chloroethyl vinyl ether

Chloroform

Chloromethane (Methyl chloride)

Chromium

Dibromochloromethane

1,1-Dichloroethane

1,2-Dichloroethane

cis-1,2-Dichloroethene

1,1-Dichloroethylene

Dichloromethane (Methylene chloride)

2,4-Dichlorophenoxyacetic acid

1,2-Dichloropropane

cis-1,3-Dichloropropene

trans-1,3-Dichloropropene

Endrin

Ethylbenzene

Fluoride

Iron

Lead

Lindane

Magnesium

Manganese

Mercury

Methoxychlor

Nitrate as nitrogen

Phenols

Potassium

Selenium

Silica

Silver

Sodium

Sulfate

1,1,2,2-Tetrachloroethane

Tetrachloroethylene

Toluene

Total dissolved solids

Total organic carbon

Total organic halogens

Total phosphates (as P)

Toxaphene

2,4,5-TP (Silvex)

$1,1,1$-Trichloroethane

1,1,2-Trichloroethane

Trichloroethylene

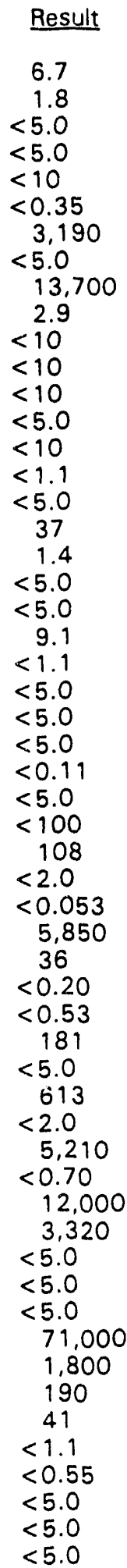

\begin{tabular}{|c|c|c|}
\hline Unit & Flag & $\underline{L a b}$ \\
\hline$\mu \mathrm{g} / \mathrm{L}$ & 0 & WA \\
\hline$\mu \mathrm{g} / \mathrm{L}$ & 0 & WA \\
\hline$\mu \mathrm{g} / \mathrm{L}$ & 0 & WA \\
\hline$\mu g / L$ & 0 & WA \\
\hline$\mu g / L$ & 0 & WA \\
\hline$\mu \mathrm{g} / \mathrm{L}$ & 0 & WA \\
\hline$\mu \mathrm{g} / \mathrm{L}$ & 0 & WA \\
\hline$\mu \mathrm{g} / \mathrm{L}$ & 0 & WA \\
\hline$\mu g / L$ & 0 & WA \\
\hline$\mu \mathrm{g} / \mathrm{L}$ & 0 & WA \\
\hline$\mu \mathrm{g} / \mathrm{L}$ & 0 & WA \\
\hline$\mu g / L$ & 0 & WA \\
\hline$\mu g / L$ & 0 & WA \\
\hline$\mu \mathrm{g} / \mathrm{L}$ & 0 & WA \\
\hline$\mu \mathrm{g} / \mathrm{L}$ & 0 & WA \\
\hline$\mu \mathrm{g} / \mathrm{L}$ & 0 & WA \\
\hline$\mu g / L$ & 0 & WA \\
\hline$\mu \mathrm{g} / \mathrm{L}$ & 2 & WA \\
\hline$\mu \mathrm{g} / \mathrm{L}$ & 0 & WA \\
\hline$\mu \mathrm{g} / \mathrm{L}$ & 0 & WA \\
\hline$\mu \mathrm{g} / \mathrm{L}$ & 0 & WA \\
\hline$\mu g / L$ & 0 & WA \\
\hline$\mu \mathrm{g} / \mathrm{L}$ & 0 & WA \\
\hline$\mu \mathrm{g} / \mathrm{L}$ & 0 & WA \\
\hline$\mu \mathrm{g} / \mathrm{L}$ & 0 & WA \\
\hline$\mu \mathrm{g} / \mathrm{L}$ & 0 & WA \\
\hline$\mu \mathrm{g} / \mathrm{L}$ & 0 & WA \\
\hline$\mu g / L$ & 0 & WA \\
\hline$\mu g / L$ & 0 & WA \\
\hline$\mu \mathrm{g} / \mathrm{L}$ & 0 & WA \\
\hline$\mu \mathrm{g} / \mathrm{L}$ & 0 & WA \\
\hline$\mu \mathrm{g} / \mathrm{L}$ & 0 & WA \\
\hline$\mu \mathrm{g} / \mathrm{L}$ & 0 & WA \\
\hline$\mu \mathrm{g} / \mathrm{L}$ & 1 & WA \\
\hline$\mu \mathrm{g} / \mathrm{L}$ & 0 & WA \\
\hline$\mu \mathrm{g} / \mathrm{L}$ & 0 & WA \\
\hline$\mu \mathrm{g} / \mathrm{L}$ & 0 & WA \\
\hline$\mu \mathrm{g} / \mathrm{L}$ & 0 & WA \\
\hline$\mu \mathrm{g} / \mathrm{L}$ & 0 & WA \\
\hline$\mu g / L$ & 0 & $W A$ \\
\hline$\mu \mathrm{g} / \mathrm{L}$ & 0 & WA \\
\hline$\mu \mathrm{g} / \mathrm{L}$ & 0 & WA \\
\hline$\mu \mathrm{g} / \mathrm{L}$ & 0 & WA \\
\hline$\mu \mathrm{g} / \mathrm{L}$ & 0 & WA \\
\hline$\mu \mathrm{g} / \mathrm{L}$ & 0 & WA \\
\hline$\mu \mathrm{g} / \mathrm{L}$ & 0 & WA \\
\hline$\mu \mathrm{g} / \mathrm{L}$ & 0 & WA \\
\hline$\mu \mathrm{g} / \mathrm{L}$ & 0 & WA \\
\hline$\mu \mathrm{g} / \mathrm{L}$ & 0 & WA \\
\hline$\mu \mathrm{g} / \mathrm{L}$ & 2 & WA \\
\hline$\mu \mathrm{g} / \mathrm{L}$ & 0 & WA \\
\hline$\mu \mathrm{g} / \mathrm{L}$ & 0 & WA \\
\hline$\mu \mathrm{g} / \mathrm{L}$ & 0 & WA \\
\hline$\mu \mathrm{g} / \mathrm{L}$ & 0 & WA \\
\hline$\mu g / L$ & 0 & WA \\
\hline$\mu \mathrm{g} / \mathrm{L}$ & 0 & WA \\
\hline
\end{tabular}

$\overline{-~=~ e x c e e d e d ~ h o l d i n g ~ t i m e . ~}$

- exceeded primary drinking water standard. 
WELL LFW 57D collected on 06/08/92, laboratory analyses (cont.)

$\begin{array}{lllll}\text { H } \underline{\mathrm{D}} \text { Analyte } & \underline{\text { Result }} & \underline{\text { Unit }} & \underline{\text { Flag }} & \underline{\mathrm{Lab}} \\ \text { Trichlorofluoromethane } & <5.0 & \mu \mathrm{g} / \mathrm{L} & 0 & \text { WA } \\ \text { Gross alpha } & <3.0 \mathrm{E}+00 & \mathrm{pCi} / \mathrm{L} & 0 & \mathrm{CN} \\ \text { Nonvolatile beta } & <5.0 \mathrm{E}+00 & \mathrm{pCi} / \mathrm{L} & 0 & \mathrm{CN} \\ \text { Radium-226 } & <5.0 \mathrm{E}-01 & \mathrm{pCi} / \mathrm{L} & 0 & \mathrm{CN} \\ \text { Tritium } & 4.2 \mathrm{E}+00 \pm 4.7 \mathrm{E}-01 & \mathrm{pCi} / \mathrm{mL} & 0 & \mathrm{CN}\end{array}$

WELL LFW 58D

$\begin{array}{llllll}\text { SRS Coord. } & \text { Lat/Longitude } & \text { Screen Zone Elevation } & \text { Top of Casing } & \text { Casing } & \text { Formation } \\ \text { N82940.6 } & 33.283771^{\circ} \mathrm{N} & 147.6-127.5 \mathrm{ft} \mathrm{msl} & 167.6 \mathrm{ft} \mathrm{msl} & \text { 4" PVC } \\ \text { E45700.2 } & 81.707573^{\circ} \mathrm{W} & & & \end{array}$

\section{MEASUREMENTS CONDUCTED IN THE FIELD}

Sample date: 06/08/92

Depth to water: 26.25 ft $(8.00 \mathrm{~m})$ below TOC

Water elevation: $141.35 \mathrm{ft}(43.08 \mathrm{~m}) \mathrm{msl}$

Sp. conductance: $72 \mu \mathrm{S} / \mathrm{cm}$

Water evacuated before sampling: $36 \mathrm{gal}$

\section{LABORATORY ANALYSES}

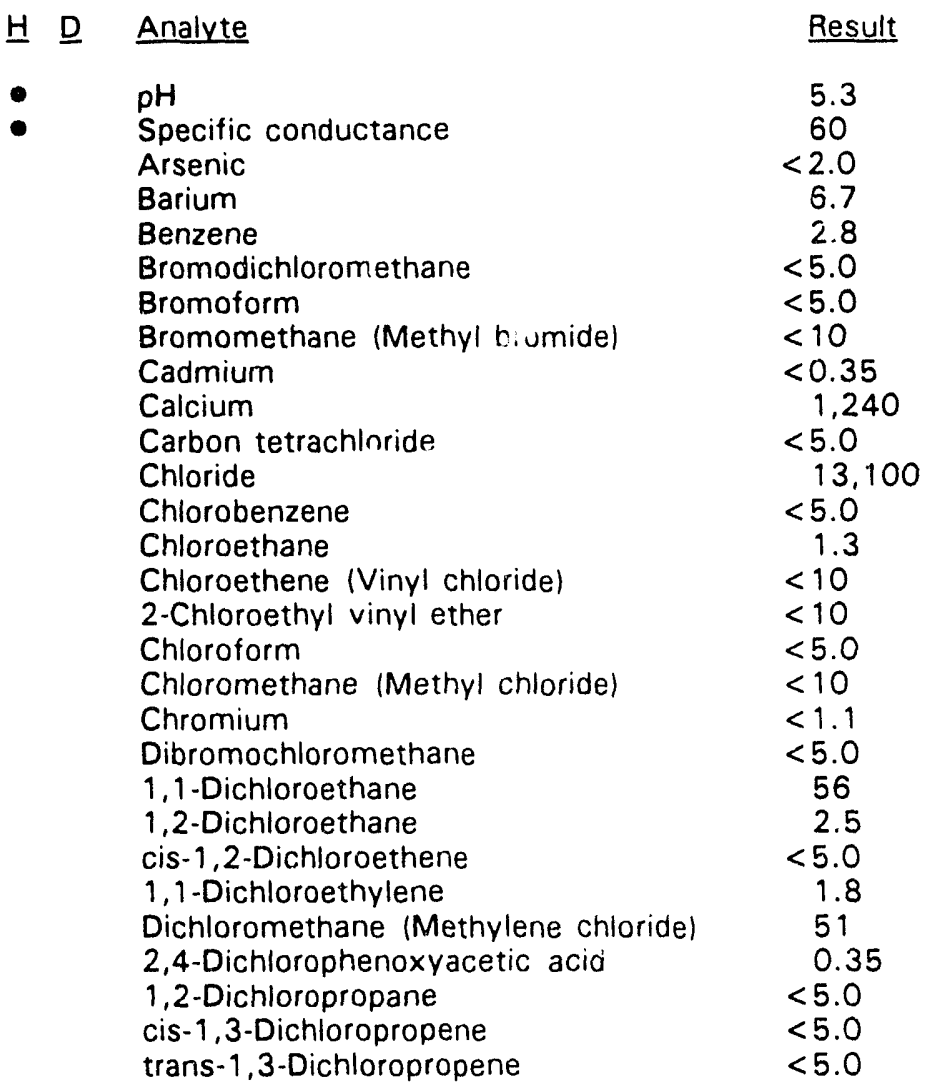

Time: $12: 00$

$\mathrm{pH}: 4.4$

Alkalinity: $0 \mathrm{mg} / \mathrm{L}$

Water temperature: $19.3^{\circ} \mathrm{C}$ 
WELL LFW 58D collected on 06/08/92, laboratory analyses (cont.)

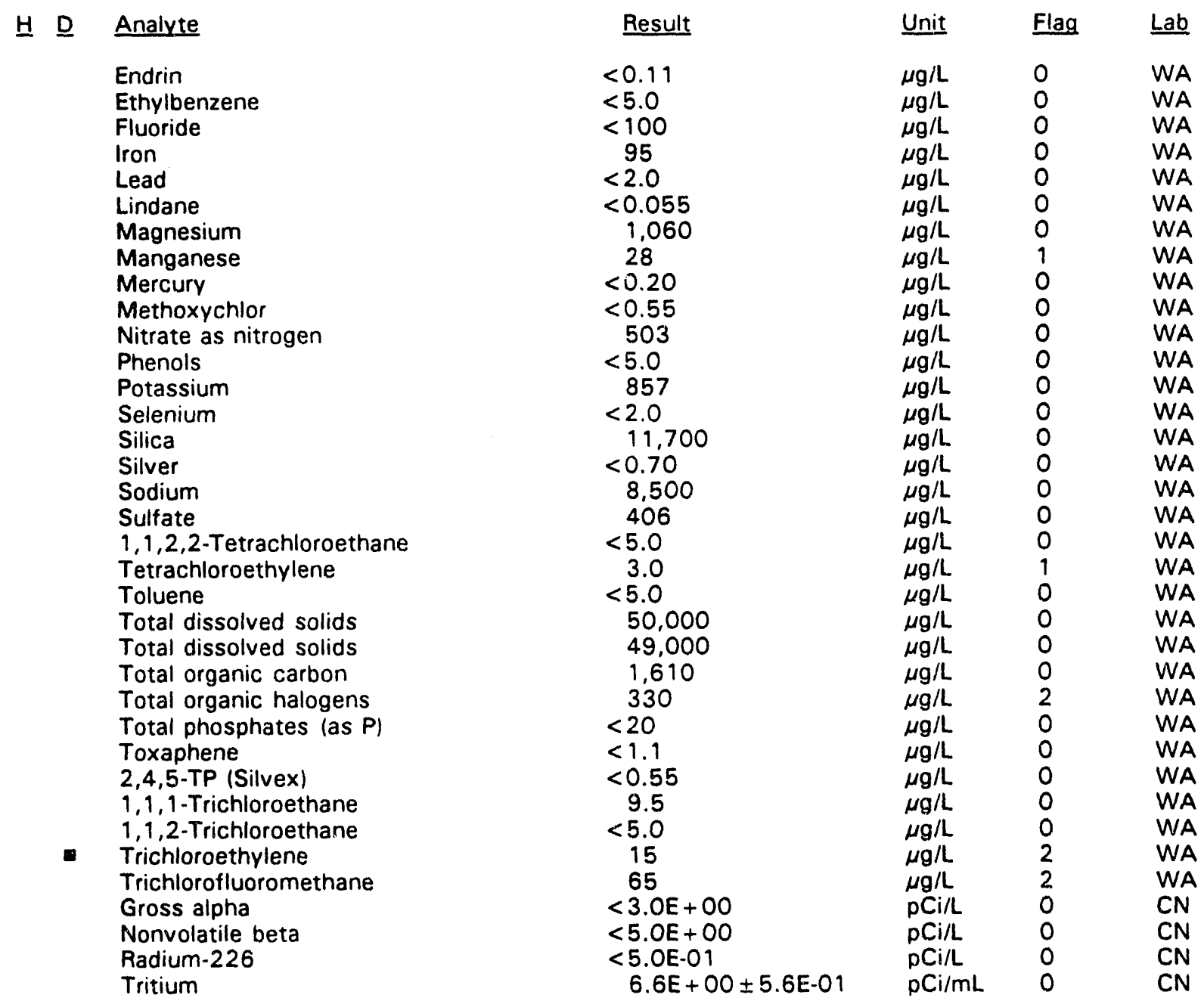

- = exceeded holding time. = exceeded primary drinking water standard. 
WELL LFW 59B

$\begin{array}{llllll}\text { SRS Coord. } & \text { Lat/Longitude } & \text { Screen Zone Elevation } & \text { Top of Casing } & \text { Casing } & \text { Formation } \\ \text { N83027.1 } & 33.284529^{\circ} \mathrm{N} & 76.0-66.0 \mathrm{ft} \mathrm{msl} & & 168.1 \mathrm{ft} \mathrm{msl} & 4 \text { " PVC } \\ \text { E46047.4 } & 81.706827^{\circ} \mathrm{W} & & & \end{array}$

\section{MEASUREMENTS CONDUCTED IN THE FIELD}

Sample date: 06/05/92

Depth to water: $25.84 \mathrm{ft}(7.88 \mathrm{~m})$ below TOC

Water elevation: $142.26 \mathrm{ft}(43.36 \mathrm{~m}) \mathrm{ms}$ l

Sp. conductance: $56 \mu \mathrm{S} / \mathrm{cm}$

Water evacuated before sampling: $201 \mathrm{gal}$

Time: $15: 00$

pH: 3.9

Alkalinity: $0 \mathrm{mg} / \mathrm{L}$

Water temperature: $20.3^{\circ} \mathrm{C}$

\section{LABORATORY ANALYSES}

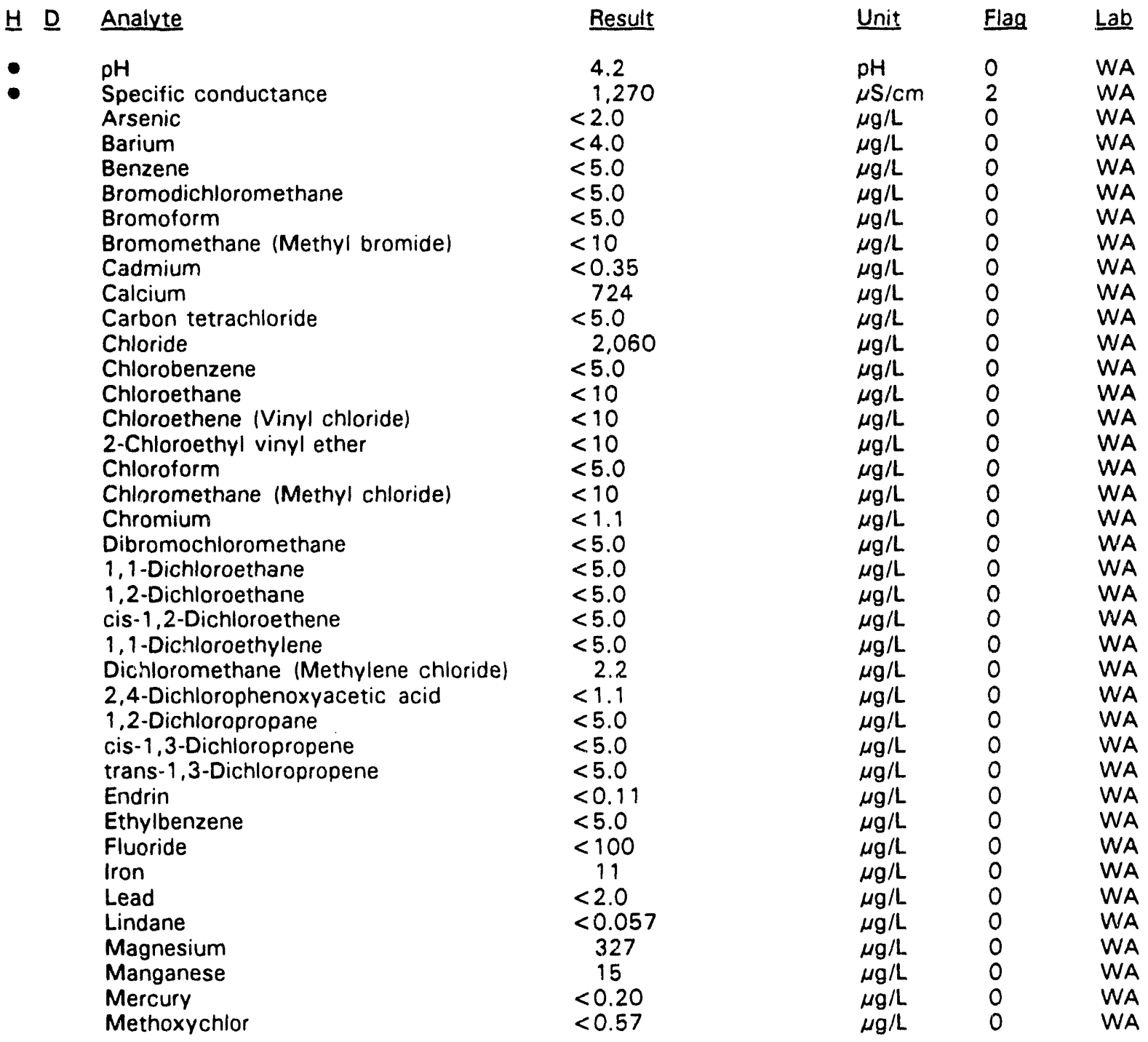

$\overline{0}$ = exceeded holding time. $\mathbf{a}=$ exceeded primary drinking water standard. 
WELL LFW 59B collected on 06/05/92, laboratory analyses (cont.)

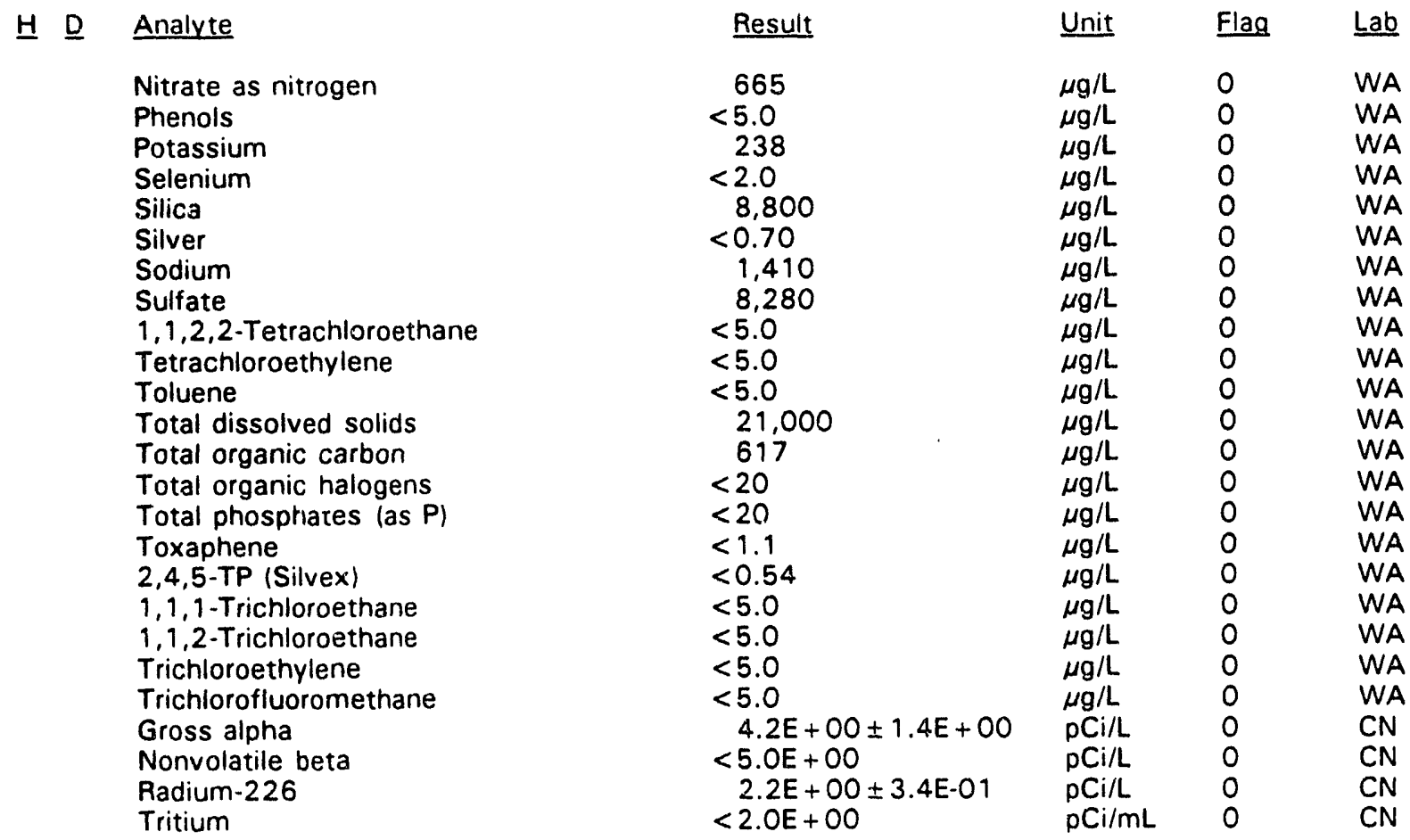

\section{WELL LFW 59C}

$\begin{array}{llllll}\text { SRS Coord } & \text { Lat/Longitude } & \text { Screen Zone Elevation } & \text { Top of Casing } & \text { Casing } & \text { Formation } \\ \text { N83011.0 } & 33.284501^{\circ} \mathrm{N} & 110.3-100.3 \mathrm{ft} \mathrm{msl} & & 167.3 \mathrm{ft} \mathrm{msl} & \text { 4" PVC } \\ \text { E46052.0 } & 81.706784^{\circ} \mathrm{W} & & & \end{array}$

\section{MEASUREMENTS CONDUCTED IN THE FIELD}

Sample date: 06/05/92

Depth to water: $24.86 \mathrm{ft}(7.58 \mathrm{~m})$ below TOC

Water elevation: $142.44 \mathrm{ft}(43.42 \mathrm{~m}) \mathrm{ms}$ l

Sp. conductance: $41 \mu \mathrm{S} / \mathrm{cm}$

Water evacuated before sampling: $112 \mathrm{gal}$

\section{LABORATORY ANALYSES}

Time: $14: 10$

$\mathrm{pH}: 4.4$

Alkalinity: $0 \mathrm{mg} / \mathrm{L}$

Water temperature: $20.0^{\circ} \mathrm{C}$

\begin{tabular}{|c|c|c|c|c|c|}
\hline H $\underline{D}$ & Analyte & Result & Unit & Flag & $\underline{\text { Lab }}$ \\
\hline & $\begin{array}{l}\mathrm{pH} \\
\text { Specific conductance } \\
\text { Arsenic } \\
\text { Barium } \\
\text { Benzene } \\
\text { Bromodichloromethane } \\
\text { Bromoform } \\
\text { Bromomethane (Methyl bromide) } \\
\text { Cadmium }\end{array}$ & $\begin{aligned} & 4.8 \\
& 36 \\
< & 2.0 \\
& 7.2 \\
< & 5.0 \\
< & 5.0 \\
< & 5.0 \\
< & 10 \\
< & 0.35\end{aligned}$ & $\begin{array}{l}\mathrm{pH} \\
\mu \mathrm{S} / \mathrm{cm} \\
\mu \mathrm{g} / \mathrm{L} \\
\mu \mathrm{g} / \mathrm{L} \\
\mu \mathrm{g} / \mathrm{L} \\
\mu \mathrm{g} / \mathrm{L} \\
\mu \mathrm{g} / \mathrm{L} \\
\mu \mathrm{g} / \mathrm{L} \\
\mu \mathrm{g} / \mathrm{L}\end{array}$ & $\begin{array}{l}0 \\
0 \\
0 \\
0 \\
0 \\
0 \\
0 \\
0 \\
0\end{array}$ & $\begin{array}{l}\text { WA } \\
\text { WA } \\
\text { WA } \\
\text { WA } \\
\text { WA } \\
\text { WA } \\
\text { WA } \\
\text { WA } \\
\text { WA }\end{array}$ \\
\hline
\end{tabular}

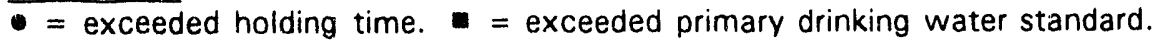


WELL LFW 59C collected on 06/05/92, labora analyses (cont.)

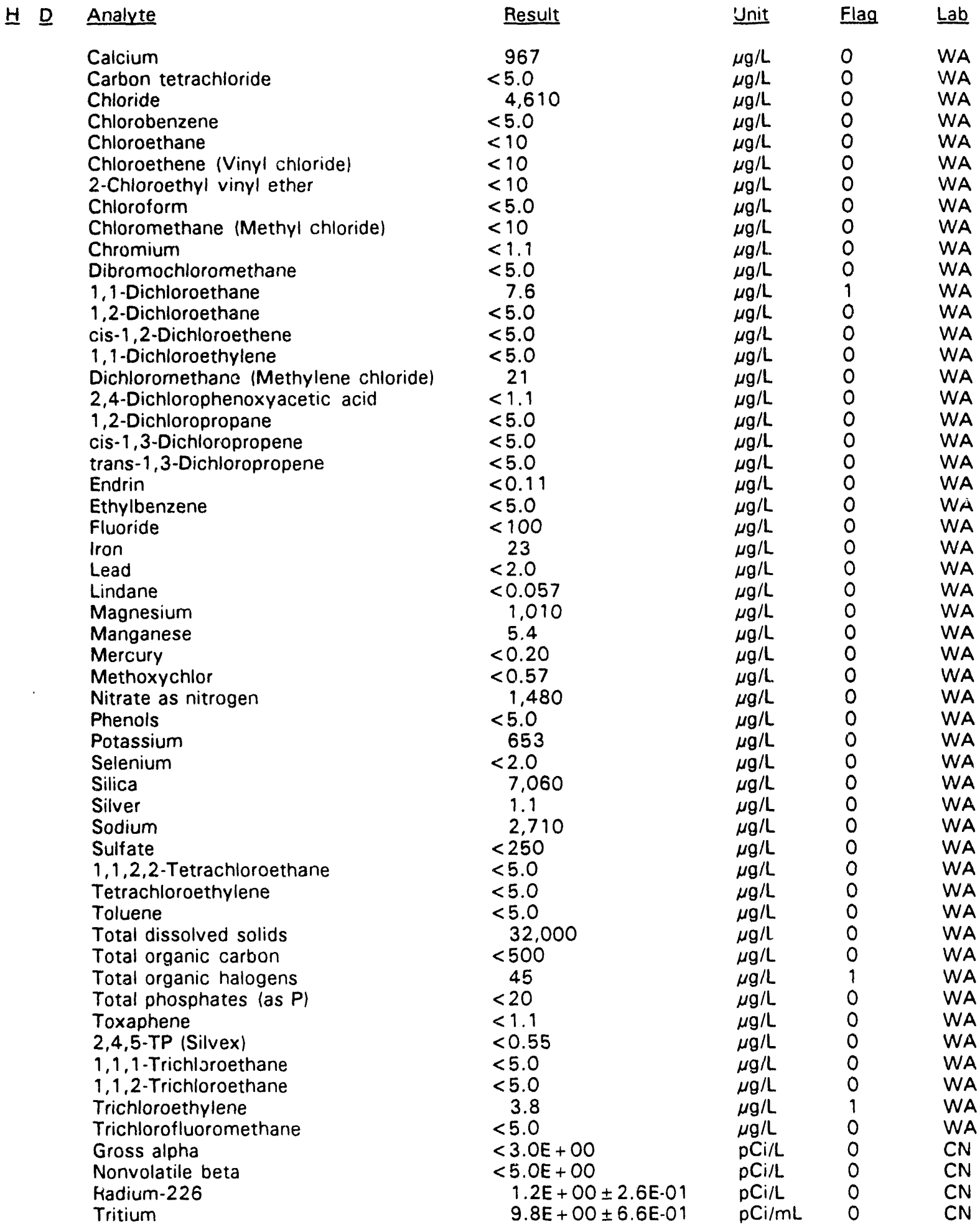

$\overline{0}=$ exceeded holding time. = exceeded primary drinking water standard. 
WELL LFW 59D

$\begin{array}{llllll}\text { SRS Coord. } & \text { Lat/Longitude } & \text { Screen Zone Elevation } & \text { Top of Casing } & \text { Casing } & \text { Formation } \\ \text { N83000.1 } & 33.284484 & \mathrm{o} & 149.3-129.3 \mathrm{ft} \mathrm{msl} & 167.6 \mathrm{ft} \mathrm{msl} & 4 \text { " PVC } \\ \text { E46056.1 } & 81.706752^{\circ} \mathrm{W} & & & \end{array}$

MEASUREMENTS CONDUCTED IN THE FIELD

Sample date: 06/05/92

Depth to water: $25.48 \mathrm{ft}(7.77 \mathrm{~m})$ below TOC Water elevation: $142.12 \mathrm{ft}(43.32 \mathrm{~m}) \mathrm{msl}$

Sp. conductance: $37 \mu \mathrm{S} / \mathrm{cm}$

Water evacuated before sampling: $34 \mathrm{gal}$

\section{LABORATORY ANALYSES}

\begin{tabular}{lc}
\hline D & Result \\
Analyte & \\
pH & 5.7 \\
Specific conductance & 45 \\
Arsenic & $<2.0$ \\
Barium & 6.8 \\
Benzene & 1.1 \\
Bromodichloromethane & $<5.0$ \\
Bromoform & $<5.0$ \\
Bromomethane (Methyl bromide) & $<10$ \\
Cadmium & 0.55 \\
Calcium & 872 \\
Carbon tetrachloride & $<5.0$ \\
Chloride & 8.190 \\
Chlorobenzene & $<5.0$ \\
Chloroethane & $<10$ \\
Chloroethene (Vinyl chloride) & $<10$ \\
2-Chloroethyl vinyl ether & $<10$ \\
Chloroform & $<5.0$ \\
Chloromethane (Methyl chloride) & $<10$ \\
Chromium & $<1.1$ \\
Dibromochloromethane & $<5.0$ \\
1.1-Dichloroethane & 35 \\
1,2-Dichloroethane & 1.6 \\
cis-1,2-Dichloroethene & $<5.0$ \\
1.1-Dichloroethylene & 1.3 \\
Dichloromethane (Methylene chloride) & 42 \\
2,4-Dichlorophenoxyacetic acid & $<1.1$ \\
1.2-Dichloropropane & $<5.0$ \\
cis-1,3-Dichloropropene & $<5.0$ \\
trans-1,3-Dichloropropene & $<5.0$ \\
Endrin & $<0.11$ \\
Ethylbenzene & $<5.0$ \\
Fluoride & $<100$ \\
Iron & 39 \\
Lead & 2.8 \\
Lindane & $<0.057$ \\
Magnesium & 866 \\
Manganese & 17 \\
Mercury & $<0.20$ \\
Methoxychlor & \\
& $<0.57$
\end{tabular}

Time: $14: 25$

$\mathrm{pH}: 4.6$

Alkalinity: $0 \mathrm{mg} / \mathrm{L}$

Water temperature: $19.4^{\circ} \mathrm{C}$ 
WELL LFW 59D collected on 06/05/92, laboratory analyses (cont.)

$\begin{array}{ll}\text { H. } & \text { Analyte } \\ \text { Nitrate as nitrogen } \\ \text { Phenols } \\ \text { Potassium } \\ \text { Selenium } \\ \text { Silica } \\ \text { Silver } \\ \text { Sodium } \\ \text { Sulfate } \\ \text { 1,1,2,2-Tetrachloroethane } \\ \text { Tetrachloroethylene } \\ \text { Toluene } \\ \text { Total dissolved solids } \\ \text { Totai organic carbon } \\ \text { Totai organic halogens } \\ \text { Total phosphates las P) } \\ \text { Toxaphene } \\ \text { 2,4,5-TP (Silvex) } \\ \text { 1,1,1-Trichloroethane } \\ \text { 1,1,2-Trichloroethane } \\ \text { Trichloroethylene } \\ \text { Trichlorofluoromethane } \\ \text { Gross alpha } \\ \text { Nonvolatile beta } \\ \text { Radium-226 } \\ \text { Radium-226 } \\ \text { Tritium }\end{array}$

$\begin{aligned} & \text { Result } \\ & 213 \\ &< 5.0 \\ & 357 \\ &< 2.0 \\ & 8.410 \\ &< 0.70 \\ & 3.620 \\ &< 250 \\ &<5.0 \\ &<5.0 \\ &<5.0 \\ & 30,000 \\ & 607 \\ & 209 \\ &<20 \\ &<1.1 \\ &<0.56 \\ & 3.7 \\ &<5.0 \\ & 14 \\ &<5.0 \\ &<3.0 \mathrm{E}+00 \\ &<5.0 \mathrm{E}+00 \\ &<5.0 \mathrm{E}-01 \\ &<5.0 \mathrm{E}-01 \\ & 8.1 \mathrm{E}+00 \pm 6.1 \mathrm{E}-01\end{aligned}$

$\begin{array}{lll}\text { Unit } & \text { Flag } & \text { Lab } \\ \mu \mathrm{g} / \mathrm{L} & 0 & \text { WA } \\ \mu \mathrm{g} / \mathrm{L} & 0 & \text { WA } \\ \mu \mathrm{g} / \mathrm{L} & 0 & \text { WA } \\ \mu \mathrm{g} / \mathrm{L} & 0 & \text { WA } \\ \mu \mathrm{g} / \mathrm{L} & 0 & \text { WA } \\ \mu \mathrm{g} / \mathrm{L} & 0 & \text { WA } \\ \mu \mathrm{g} / \mathrm{L} & 0 & \text { WA } \\ \mu \mathrm{g} / \mathrm{L} & 0 & \text { WA } \\ \mu \mathrm{g} / \mathrm{L} & 0 & \text { WA } \\ \mu \mathrm{g} / \mathrm{L} & 0 & \text { WA } \\ \mu \mathrm{g} / \mathrm{L} & 0 & \text { WA } \\ \mu \mathrm{g} / \mathrm{L} & 0 & \text { WA } \\ \mu \mathrm{g} / \mathrm{L} & 0 & \text { WA } \\ \mu \mathrm{g} / \mathrm{L} & 2 & \text { WA } \\ \mu \mathrm{g} / \mathrm{L} & 0 & \text { WA } \\ \mu \mathrm{g} / \mathrm{L} & 0 & \text { WA } \\ \mu \mathrm{g} / \mathrm{L} & 0 & \text { WA } \\ \mu \mathrm{g} / \mathrm{L} & 0 & \text { WA } \\ \mu \mathrm{g} / \mathrm{L} & 0 & \text { WA } \\ \mu \mathrm{g} / \mathrm{L} & 2 & \text { WA } \\ \mu \mathrm{g} / \mathrm{L} & 0 & \text { WA } \\ \mathrm{pCi} / \mathrm{L} & 0 & \mathrm{CN} \\ \mathrm{pCi} / \mathrm{L} & 0 & \mathrm{CN} \\ \mathrm{pCi} / \mathrm{L} & 0 & \mathrm{CN} \\ \mathrm{pCi} / \mathrm{L} & 0 & \mathrm{CN} \\ \mathrm{pC} \mathrm{Ci} / \mathrm{mL} & 0 & \mathrm{CN}\end{array}$

WA

WA

WA

WA WA

WA WA

WA WA

WA WA

WA WA

WA

WA

CN

$\mathrm{CN}$

CN

\section{WELL LFW 60D}

\begin{tabular}{|c|c|c|c|c|c|}
\hline SRS Coord. & Lat/Longitude & Screen Zone Elevation & Top of Casing & Casing & Formation \\
\hline $\begin{array}{l}\text { N82531.5 } \\
E 45722.3\end{array}$ & $\begin{array}{l}33.282903^{\circ} \mathrm{N} \\
81.706720^{\circ} \mathrm{W}\end{array}$ & $143.8-123.8 \mathrm{ft} \mathrm{msl}$ & $157.1 \mathrm{ft} \mathrm{msl}$ & 4" PVC & \\
\hline
\end{tabular}

\section{MEASUREMENTS CONDUCTED IN THE FIELD}

Sample date: 06/05/92

Depth to water: $19.14 \mathrm{ft}\langle 5.83 \mathrm{~m}\rangle$ below TOC Water elevation: $137.96 \mathrm{ft}(42.05 \mathrm{~m}) \mathrm{msl}$

Sp. conductance: $20 \mu \mathrm{S} / \mathrm{cm}$

Water evacuated before sampling: $11 \mathrm{gal}$

The well went dry during purging.

\section{LABORATORY ANALYSES}

H $\underline{\text { Analyte }}$

-

$\mathrm{pH}$
Specific conductance

Arsenic

Barium

Benzene

Bromodichloromethane

Bromoform

Bromomethane (Methyl bromide)
Result

$\begin{aligned} & 4.8 \\ & 19 \\ < & 2.0 \\ < & 4.0 \\ < & 5.0 \\ < & 5.0 \\ < & 5.0 \\ < & 10\end{aligned}$

Time: $11: 10$

$\mathrm{pH}: 4.7$

Alkalinity: $7 \mathrm{mg} / \mathrm{L}$

Water temperature: $19.2^{\circ} \mathrm{C}$ 
WELL LFW 60D collected on 06/05/92, laboratory analyses (cont.)

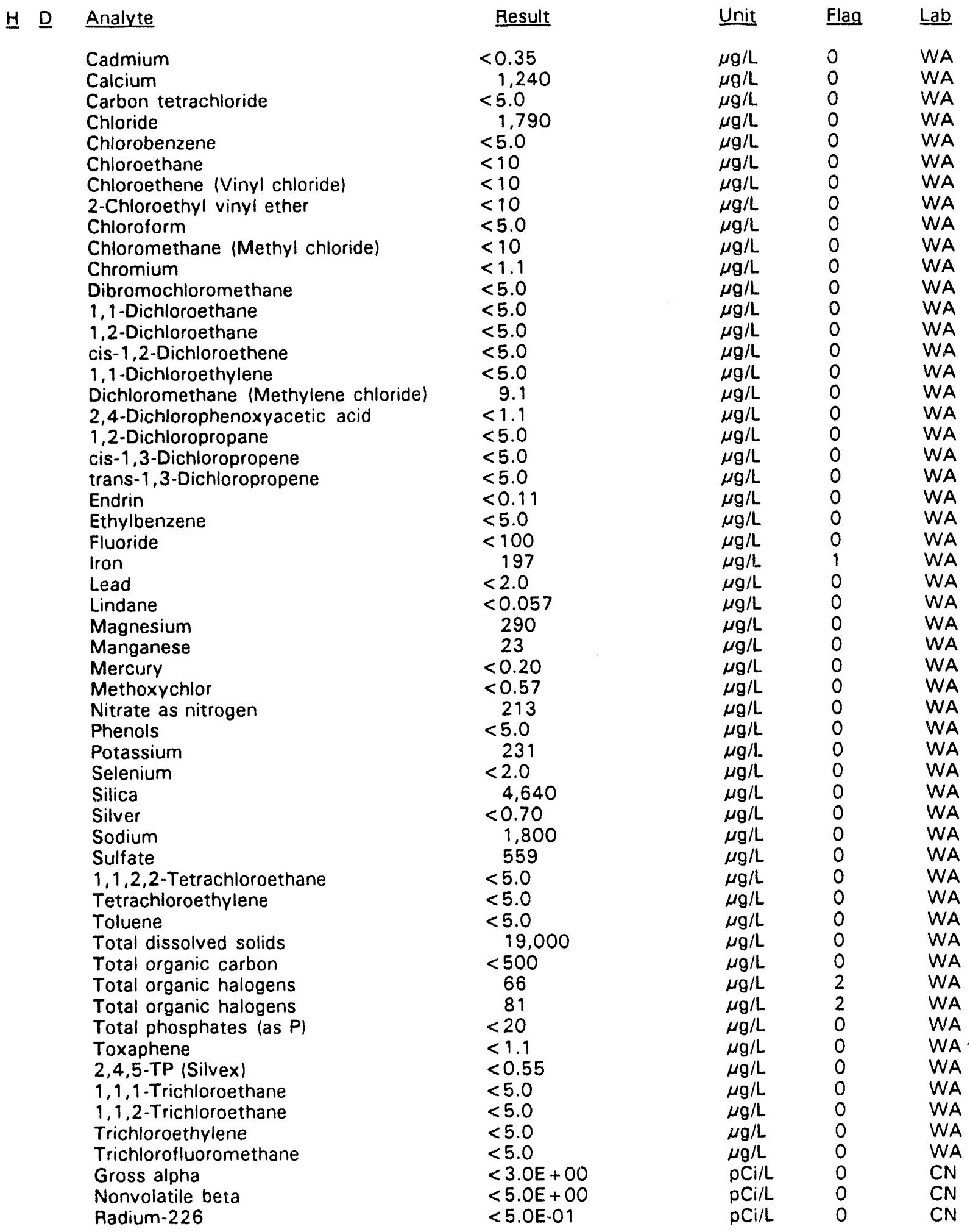

- = exceeded holding time. $\square=$ exceeded primary drinking water standard. 
WELL LFW 60D coliecteư on 06/05/92, laboratory analyses (cont.)

\begin{tabular}{|c|c|c|c|}
\hline Analyte & Result & Unit & Flag \\
\hline Tritium & $<2.0 E+00$ & $\mathrm{pCi} / \mathrm{mL}$ & 0 \\
\hline
\end{tabular}

WELL LFW 61C

$\begin{array}{llllll}\text { SRS Coord. } & \text { Lat/Longitude } & \text { Screen Zone Elevation } & \text { Top of Casing } & \text { Casing } & \text { Formation } \\ \text { N83084.4 } & 33.285378^{\circ} \mathrm{N} & 121.1-111.0 \mathrm{ft} \mathrm{msl} & 168.3 \mathrm{ft} \mathrm{msl} & 4^{\text {" PVC }} \\ \text { E46489.6 } & 81.705774^{\circ} \mathrm{W} & & & \end{array}$

MEASUREMENTS CONDUCTED IN THE FIELD

Sample date: 04/28/92

Depth to water: $26.95 \mathrm{ft}(8.21 \mathrm{~m})$ below TOC

Water elevation: $141.35 \mathrm{ft}(43.08 \mathrm{~m}) \mathrm{msl}$

Sp. conductance: $39 \mu \mathrm{S} / \mathrm{cm}$

Water evacuated before sampling: $80 \mathrm{gal}$

\section{LABORATORY ANALYSES}

H D Analyte

$\mathrm{pH}$

Specific conductance

Arsenic

Barium

Benzene

Bromodichloromethane

Bromoform

Bromomethane (Methyl bromide)

Cadmium

Calcium

Carbon tetrachloride

Chiloride

Chloride

Chlorobenzene

Chloroethane

Chloroethene (Vinyl chloride)

2-Chloroethyl vinul ether

Chloroform

Chloromethane (Methyl chloride)

Chromium

Dibromochloromethane

1,1-Dichloroethane

1,2-Dichloroethane

1,1-Dichloroethylene

trans-1,2-Dichloroethylene

Dichloromethane (Methylene chloride)

2,4-Dichlorophenoxyacetic acid

1,2-Dichloropropane

cis-1,3-Dichloropropene

trans-1,3-Dichloropropene

Endrin

Endrin

Ethylbenzene
Time: $10: 50$

$\mathrm{pH}: 5.0$

Alkalinity: $0 \mathrm{mg} / \mathrm{L}$

Water temperature: $18.4^{\circ} \mathrm{C}$

\begin{tabular}{llll} 
Result & Unit & Flag & Lab \\
\hline & & & \\
5.2 & $\mathrm{pH}$ & 0 & WA \\
34 & $\mu \mathrm{S} / \mathrm{cm}$ & 0 & WA \\
$<2.0$ & $\mu \mathrm{g} / \mathrm{L}$ & 0 & WA \\
7.5 & $\mu \mathrm{g} / \mathrm{L}$ & 0 & WA \\
$<5.0$ & $\mu \mathrm{g} / \mathrm{L}$ & 0 & WA \\
$<5.0$ & $\mu \mathrm{g} / \mathrm{L}$ & 0 & WA \\
$<5.0$ & $\mu \mathrm{g} / \mathrm{L}$ & 0 & WA \\
$<10$ & $\mu \mathrm{g} / \mathrm{L}$ & 0 & WA \\
0.47 & $\mu \mathrm{g} / \mathrm{L}$ & 0 & WA \\
982 & $\mu \mathrm{g} / \mathrm{L}$ & 0 & WA \\
$<5.0$ & $\mu \mathrm{g} / \mathrm{L}$ & 0 & WA \\
6.280 & $\mu \mathrm{g} / \mathrm{L}$ & 0 & WA \\
6.310 & $\mu \mathrm{g} / \mathrm{L}$ & 0 & WA \\
$<5.0$ & $\mu \mathrm{g} / \mathrm{L}$ & 0 & WA \\
17 & $\mu \mathrm{g} / \mathrm{L}$ & 2 & WA \\
$<10$ & $\mu \mathrm{g} / \mathrm{L}$ & 0 & WA \\
$<10$ & $\mu \mathrm{g} / \mathrm{L}$ & 0 & WA \\
$<5.0$ & $\mu \mathrm{g} / \mathrm{L}$ & 0 & WA \\
$<10$ & $\mu \mathrm{g} / \mathrm{L}$ & 0 & WA \\
$<1.1$ & $\mu \mathrm{g} / \mathrm{L}$ & 0 & WA \\
$<5.0$ & $\mu \mathrm{g} / \mathrm{L}$ & 0 & WA \\
32 & $\mu \mathrm{g} / \mathrm{L}$ & 2 & WA \\
$<5.0$ & $\mu \mathrm{g} / \mathrm{L}$ & 0 & WA \\
3.3 & $\mu \mathrm{g} / \mathrm{L}$ & 0 & WA \\
$<5.0$ & $\mu \mathrm{g} / \mathrm{L}$ & 0 & WA \\
173 & $\mu \mathrm{g} / \mathrm{L}$ & 0 & WA \\
$<1.1$ & $\mu \mathrm{g} / \mathrm{L}$ & 0 & WA \\
$<5.0$ & $\mu \mathrm{g} / \mathrm{L}$ & 0 & WA \\
$<5.0$ & $\mu \mathrm{g} / \mathrm{L}$ & 0 & WA \\
$<5.0$ & $\mu \mathrm{g} / \mathrm{L}$ & 0 & WA \\
$<0.11$ & $\mu \mathrm{g} / \mathrm{L}$ & 0 & WA \\
$<0.22$ & $\mu \mathrm{g} / \mathrm{L}$ & 0 & WA \\
18 & $\mu \mathrm{g} / \mathrm{L}$ & 0 & WA \\
& & & \\
\hline & & 0 & 0
\end{tabular}

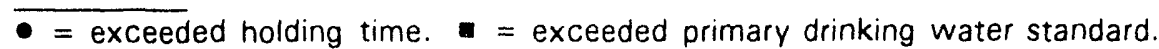


WELL LFW $61 \mathrm{C}$ collected on 04/28/92, laboratory analyses (cont.)

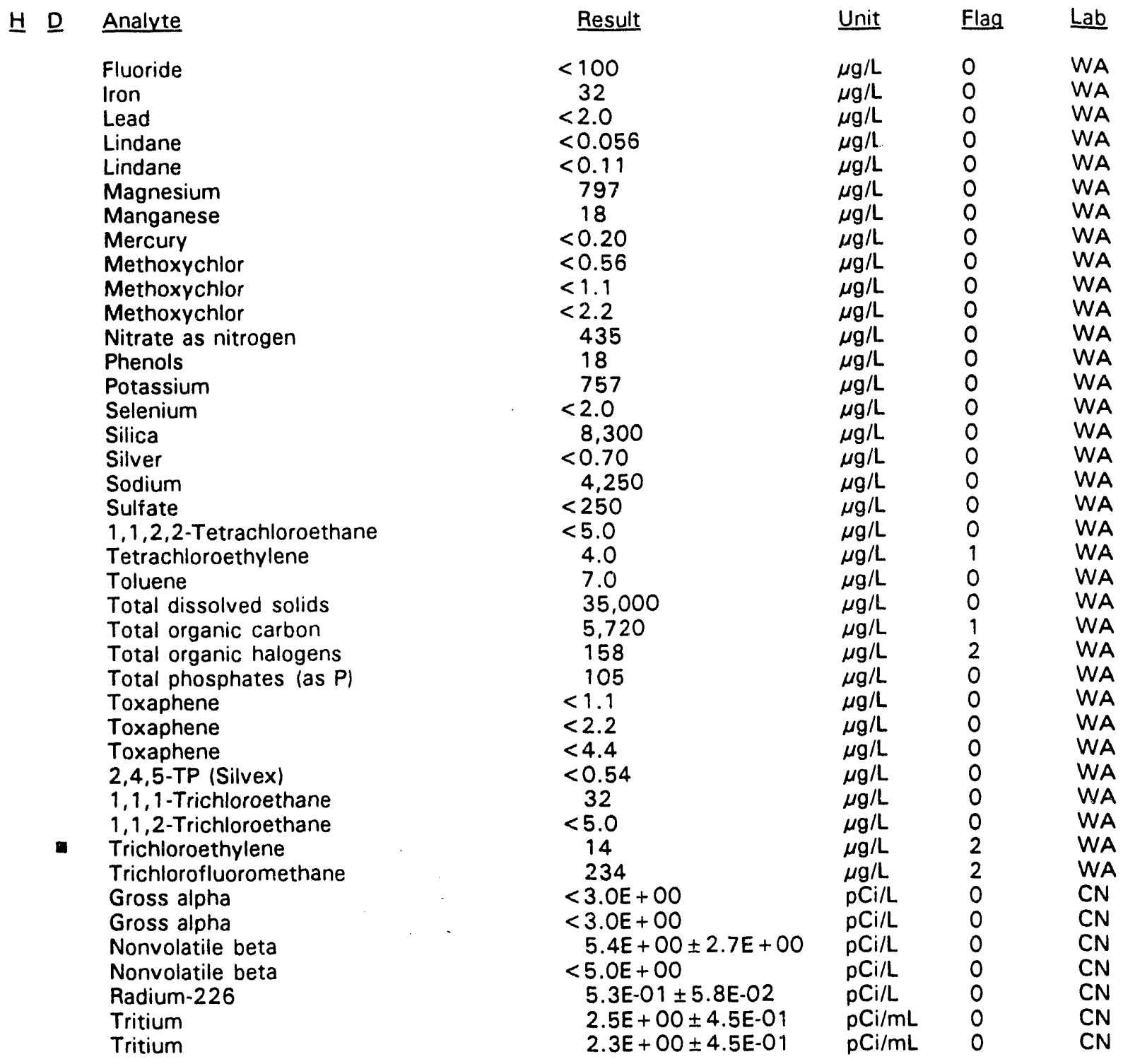

$\overline{-=\text { exceeded holding time. }}$ = exceeded primary drinking water standard. 
WELL LFW 61D

$\begin{array}{llllll}\text { SRS Coord. } & \text { Lat/Longitude } & \text { Screen Zone Elevation } & \text { Top of Casing } & \text { Casing } & \text { Formation } \\ \text { N83089.1 } & 33.285358{ }^{\circ} \mathrm{N} & 150.4-130.3 \mathrm{ft} \mathrm{msl} & 168.3 \mathrm{ft} \mathrm{msl} & \text { 4" PVC } \\ \text { E46471.1 } & 81.705832^{\circ} \mathrm{W} & & & \end{array}$

\section{MEASUREMENTS C.ONDUCTED IN THE FIELD}

Sample date: 04/28/92

Depth to water: $25.32 \mathrm{ft}(7.72 \mathrm{~m})$ below TOC

Water elevation: $142.98 \mathrm{ft}(43.58 \mathrm{~m}) \mathrm{msl}$

Sp. conductance: $20 \mu \mathrm{S} / \mathrm{cm}$

Water evacuated before sampling: 33 gal

\section{LABORATORY ANALYSES}

H D Analyte

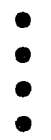

-

Specific conductance
Specific conductance

Specific conductance

Arsenic

Arsenic

Arsenic

Arsenic

Barium

Barium

Barium

Barium

- Benzene

Benzene

Benzene

Benzene

Benzene

- Bromodichloromethane

- Bromodichloromethane

Bromodichloromethane

Bromodichloromethane

Bromodichloromethane

Bromodichloromethane

- Bromoform

- Bromoform

Bromoform

Bromoform

Bromoform

Bromoform

- Bromomethane (Methyl bromide)

- Bromomethane (Methyl bromide)

Bromomethane (Methyl bromide)

Bromomethane (Methyl bromide)

Bromomethane (Methyl bromide)
Result

5.2

5.1

5.1

5.1

20

20

17

17

$<2.0$

$<2.0$

$<2.0$

$<2.0$

$<3.0$

$<3.0$

$<4.0$

$<4.0$

$<1.0$

$<1.0$

$<5.0$

$<5.0$

$<5.0$

$<1.0$

$<1.0$

$<5.0$

$<5.0$

$<5.0$

$<5.0$

$<1.0$

$<1.0$

$<5.0$

$<5.0$

$<5.0$

$<5.0$

$<1.0$

$<1.0$

$<10$

$<10$

$<10$
Time: 11:35

$\mathrm{pH}: 5.0$

Alkalinity: $0 \mathrm{mg} / \mathrm{h}$

Water temperature: $18.1^{\circ} \mathrm{C}$
Unit Flag Lab

pH $\quad 0 \quad$ GE

$\mathrm{pH} \quad \mathrm{O} \quad \mathrm{GE}$

pH $\quad 0$ WA

pH $\quad 0$ WA

$0 \quad C N$

$\mu \mathrm{S} / \mathrm{cm} \quad 0 \quad \mathrm{GE}$

$\mu \mathrm{S} / \mathrm{cm} \quad 0 \quad \mathrm{GE}$

$\mu S / \mathrm{cm} \quad 0 \quad W A$

$\mu \mathrm{S} / \mathrm{cm} \quad 0 \quad W A$

$\mu g / L \quad 0 \quad G E$

$\mu \mathrm{g} / \mathrm{L} \quad \mathrm{O} \quad \mathrm{GE}$

$\mu g / L \quad 0 \quad$ WA

$\mu g / L \quad 0 \quad$ WA

$\mu g / L \quad 0 \quad$ GE

$\mu g / L \quad 0 \quad$ GE

$\mu g / L \quad 0 \quad$ WA

$\mu g / L \quad 0 \quad$ WA

$\mu g / L \quad O \quad G E$

$\mu g / L \quad 0 \quad$ GE

$\mu g / L \quad 0 \quad W A$

$\mu g / L \quad 0 \quad$ WA

$\mu g / L \quad 0 \quad$ WA

$\mu g / L \quad 0 \quad$ GE

$\mu g / L \quad O \quad G E$

$\mu g / L \quad 0 \quad$ WA

$\mu g / L \quad 0 \quad W A$

$\mu g / L \quad 0 \quad$ WA

$\mu g / L \quad O \quad W A$

$\mu g / L \quad 0 \quad$ GE

$\mu g / L \quad O \quad G E$

$\mu g / L \quad 0 \quad$ WA

$\mu g / L \quad 0 \quad$ WA

$\mu g / L \quad 0 \quad$ WA

$\mu \mathrm{g} / \mathrm{L} \quad \mathrm{O} \quad$ WA

$\mu g / L \quad 0 \quad$ GE

$\mu \mathrm{g} / \mathrm{L} \quad 0 \quad \mathrm{GE}$

$\mu g / L \quad 0 \quad$ WA

$\mu \mathrm{g} / \mathrm{L} \quad \mathrm{WA}$

$\mu g / L \quad 0 \quad$ WA

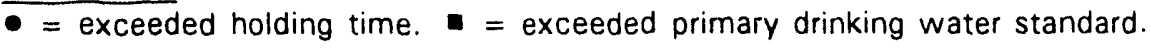


WELL LFW 610 collected on 04/28/92, laboratory analyses (cont.)

Analyte

Cadmium

$\begin{aligned} & \text { Result } \\ &< 10 \\ &< 2.0 \\ &< 2.0 \\ &< 0.35 \\ &< 0.35 \\ & 332 \\ & 331 \\ & 347 \\ & 343 \\ &<1.0 \\ &<1.0 \\ &<5.0 \\ &<5.0 \\ &<5.0 \\ &<5.0 \\ & 2.060 \\ & 2.180 \\ & 2.750 \\ & 2.950 \\ &<1.0\end{aligned}$

Unit Flag Lab

Cadmium

$\mu \mathrm{g} / \mathrm{L}$

$\mu \mathrm{g} / \mathrm{L}$

Cadmium

Cadmium

$\mu g / L$

Calcium

$\mu \mathrm{g} / \mathrm{L}$

Calcium

$\mu g / L$

Calcium

$\mu g / L$

$\mu g / L$

Calcium

$\mu \mathrm{g} / \mathrm{L}$

- Carbon tetrachloride

$\mu \mathrm{g} / \mathrm{L}$

Carbon tetrachloride

Carbon tetrachloride

Carbon tetrachloride

Carbon tetrachloride

Chloride

Chloride

Chloride

Chloride

- Chlorobenzene

Chlorobenzene

Chlorobenzene

Chlorobenzene

$<1.0$

$<5.0$

$<5.0$

$<5.0$

$<1.0$

Chloroethane

$<1.0$

$<10$

Chloroethane

$<10$

$<10$

Chloroethane

$<10$

$<1.0$

$<1.0$

$<10$

$<10$

$<10$

$<10$

$<1.0$

$<1.0$

$<10$

$<10$

$<10$

$<10$

$<1.0$

$<1.0$

$<5.0$

$<5.0$

$<5.0$

$<5.0$

$<1.0$

$<1.0$

$<10$

$<10$

$<10$

$<10$

$<4.0$

Chloromethane (Methyl chloride)

$<4.0$

$\mu g / L$

$\mu g / L$

$\mu g / L$

$\mu g / L$

$\mu g / L$

$\mu g / L$

$\mu \mathrm{g} / \mathrm{L}$

$\mu g / L$

$\mu \mathrm{g} / \mathrm{L}$

$\mu \mathrm{g} / \mathrm{L}$

$\mu g / L$

$\mu g / L$

$\mu \mathrm{g} / \mathrm{L}$

$\mu g / L$

$\mu g / L$

$\mu g / L$

$\mu \mathrm{g} / \mathrm{L}$

$\mu g / L$

$\mu \mathrm{g} / \mathrm{L}$

$\mu \mathrm{g} / \mathrm{L}$

$\mu g / L$

$\mu g / L$

$\mu g / L$

$\mu \mathrm{g} / \mathrm{L}$

$\mu g / L$

$\mu g / L$

$\mu \mathrm{g} / \mathrm{L}$

$\mu \mathrm{g} / \mathrm{L}$

$\mu g / L$

$\mu \mathrm{g} / \mathrm{L}$

$\mu \mathrm{g} / \mathrm{L}$

$\mu \mathrm{g} / \mathrm{L}$

$\mu \mathrm{g} / L$

$\mu g / L$

$\mu g / L$

$\mu g / L$

$\mu g / L$

$\mu g / L$

$\mu \mathrm{g} / \mathrm{L}$

$\mu g / L$

$\mu \mathrm{g} / \mathrm{L}$

$\mu g / L$

$\mu \mathrm{g} / \mathrm{L}$

$\mu \mathrm{g} / \mathrm{L}$

$\mu \mathrm{g} / \mathrm{L}$

Chromium

$\mu \mathrm{g} / \mathrm{L}$

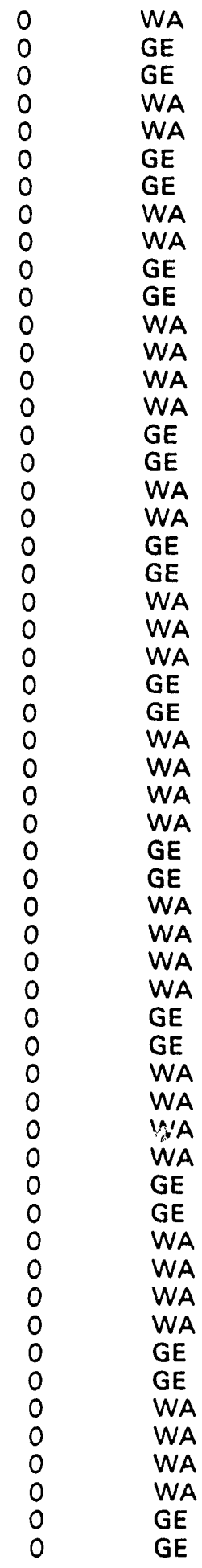

- = exceeded holding time. = exceeded primary drinking water standard. 
WELL LFW 61D collected on 04/28/92, laboratory analyses (cont.)

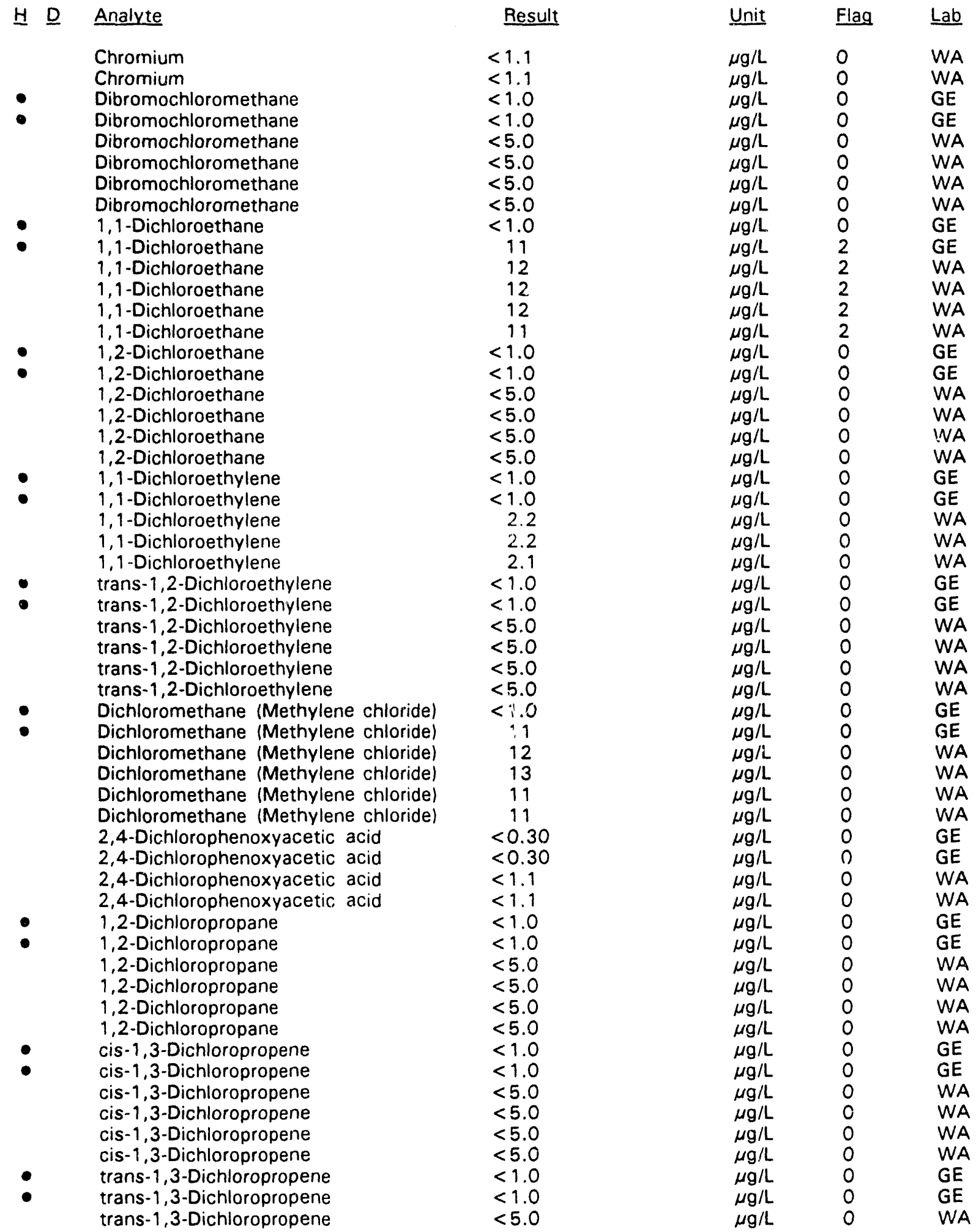

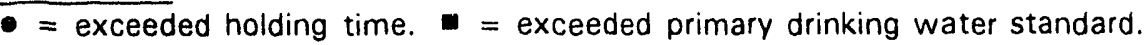


WELL LFW 610 collected on 04/28/92, laboratory analyses (cont.)

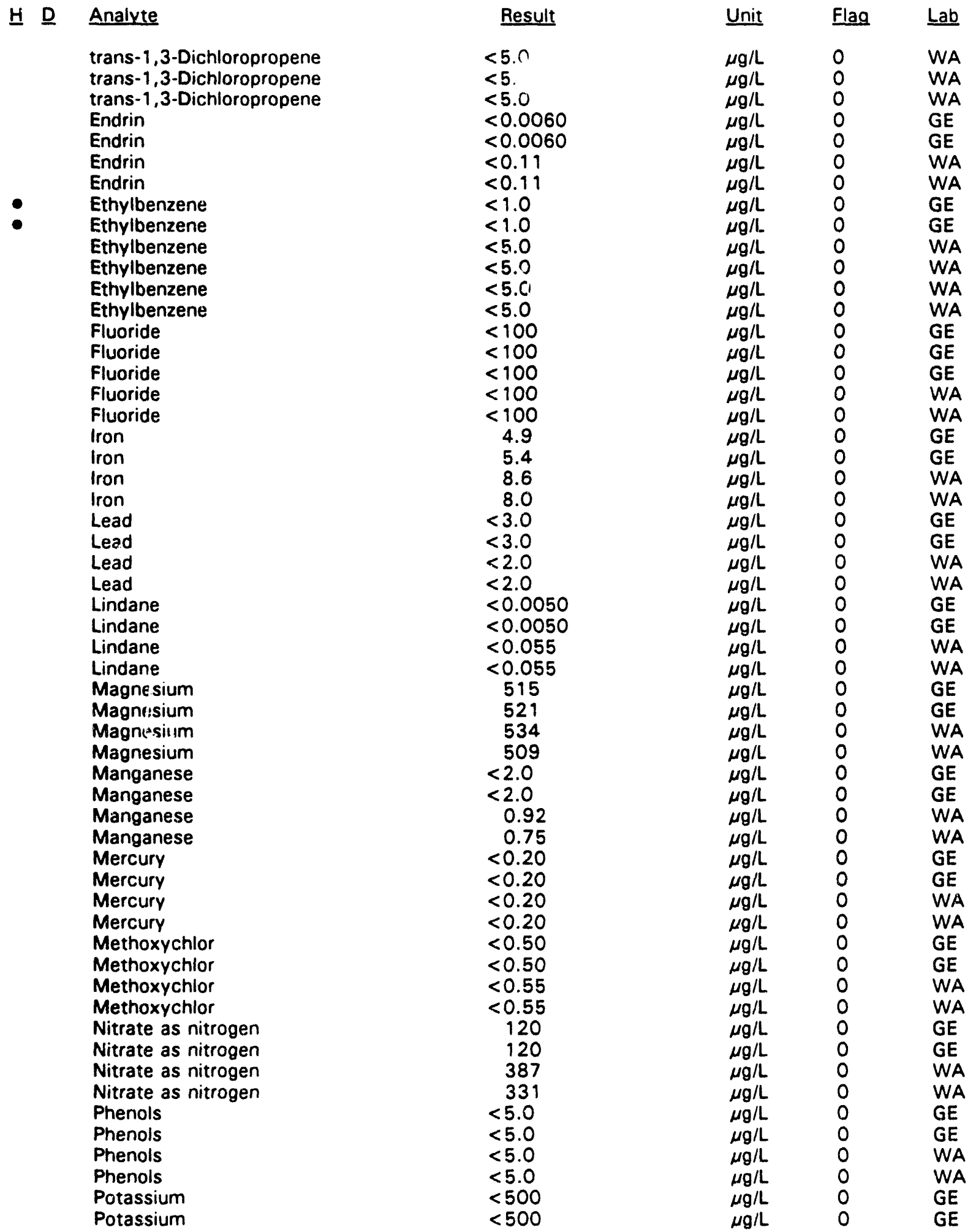

- = exceeded holding time. $\quad$ = exceeded primary drinking water standard. 
WELL LFW 61D collected on 04/28/92, laboratory analyses (cont.)

\begin{tabular}{|c|c|c|c|c|c|}
\hline \multirow[t]{2}{*}{$\underline{H} \underline{D}$} & Analyte & Result & Unit & Flag & $\underline{\text { Lab }}$ \\
\hline & $\begin{array}{l}\text { Potassium } \\
\text { Potassium } \\
\text { Selenium } \\
\text { Selenium } \\
\text { Selenium } \\
\text { Selenium } \\
\text { Silica } \\
\text { Silica } \\
\text { Silica } \\
\text { Silica } \\
\text { Silver } \\
\text { Silver } \\
\text { Silver } \\
\text { Silver } \\
\text { Sodium } \\
\text { Sodium } \\
\text { Sodium } \\
\text { Sodium } \\
\text { Sulfate } \\
\text { Sulfate } \\
\text { Sulfate } \\
\text { Sulfate } \\
\text { 1,1,2,2-Tetrachloroethane } \\
\text { 1,1,2,2-Tetrachloroethane } \\
\text { 1,1,2,2-Tetrachloroethane } \\
\text { 1,1,2,2-Tetrachloroethane } \\
1,1,2,2-\text { Tetrachloroethane } \\
\text { 1,1,2,2-Tetrachloroethane } \\
\text { Tetrachloroethylene } \\
\text { Tetrachloroethylene } \\
\text { Tetrachloroethyiene } \\
\text { Tetrachloroethylene } \\
\text { Tetrachloroethylene } \\
\text { Tetrachloroethylene } \\
\text { Toluene } \\
\text { Toluene } \\
\text { Toluene } \\
\text { Toluene } \\
\text { Toluene } \\
\text { Total dissolved solids } \\
\text { Total dissolved solids } \\
\text { Total dissolved solids } \\
\text { Total dissolved solids } \\
\text { Total organic carbon } \\
\text { Total organic carbon } \\
\text { Total organic carbon } \\
\text { Total organic carbon } \\
\text { Total organic halogens } \\
\text { Total organic halogens } \\
\text { Total organic halogens } \\
\text { Total organic halogens } \\
\text { Total organic halogens } \\
\text { Total phosphates las P) } \\
\text { Total phosphates las P) } \\
\text { Total phosphates las P) } \\
\text { Total phosphates las P) }\end{array}$ & 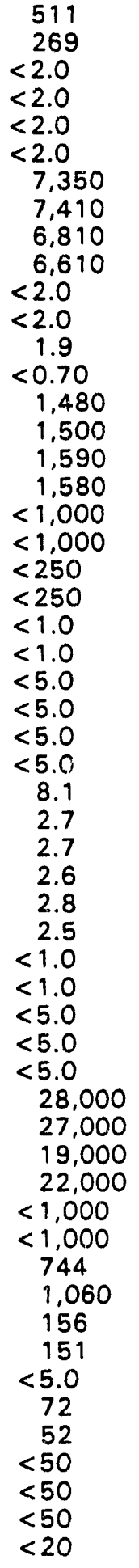 & $\begin{array}{l}\mu g / L \\
\mu g / L \\
\mu g / L \\
\mu g / L \\
\mu g / L \\
\mu g / L \\
\mu g / L \\
\mu g / L \\
\mu g / L \\
\mu g / L \\
\mu g / L \\
\mu g / L \\
\mu g / L \\
\mu g / L \\
\mu g / L \\
\mu g / L \\
\mu g / L \\
\mu g / L \\
\mu g / L \\
\mu g / L \\
\mu g / L \\
\mu g / L \\
\mu g / L \\
\mu g / L \\
\mu g / L \\
\mu g / L \\
\mu g / L \\
\mu g / L \\
\mu g / L \\
\mu g / L \\
\mu g / L \\
\mu g / L \\
\mu g / L \\
\mu g / L \\
\mu g / L \\
\mu g / L \\
\mu g / L \\
\mu g / L \\
\mu g / L \\
\mu g / L \\
\mu g / L \\
\mu g / L \\
\mu g / L \\
\mu g / L \\
\mu g / L \\
\mu g / L \\
\mu g / L \\
\mu g / L \\
\mu g / L \\
\mu g / L \\
\mu g / L \\
\mu g / L \\
\mu g / L \\
\mu g / L \\
\mu g / L \\
\mu g / L\end{array}$ & $\begin{array}{l}0 \\
0 \\
0 \\
0 \\
0 \\
0 \\
0 \\
0 \\
0 \\
0 \\
0 \\
0 \\
0 \\
0 \\
0 \\
0 \\
0 \\
0 \\
0 \\
0 \\
0 \\
0 \\
0 \\
0 \\
0 \\
0 \\
0 \\
0 \\
2 \\
1 \\
1 \\
1 \\
1 \\
1 \\
0 \\
0 \\
0 \\
0 \\
0 \\
0 \\
0 \\
0 \\
0 \\
0 \\
0 \\
0 \\
0 \\
2 \\
2 \\
0 \\
2 \\
2 \\
0 \\
0 \\
0 \\
0\end{array}$ & 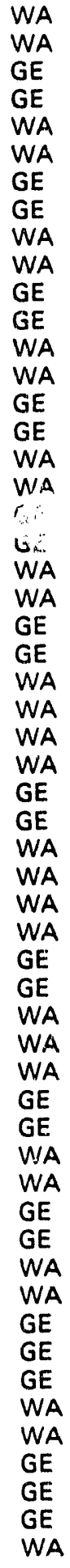 \\
\hline
\end{tabular}

\footnotetext{
$\overline{- \text { exceeded holding time. }}$ = exceeded primary drinking water standard.
} 
WELL LFW 610 collected on $04 / 28 / 92$, laboratory analyses (cont.)

H D Analyte

Total phosphates (as P)

Toxaphene

Toxaphene

Toxaphene

Toxaphene

2,4,5-TP (Silvex)

2,4,5-TP (Silvex)

2,4,5-TP (Silvex)

2,4,5-TP (Silvex)

- 1,1,1-Trichloroethane

$1,1,1$.Trichloroethane

1,1,1-Trichloroethane

1,1,1-Trichloroethane

$1,1,1$-Trichloroethane

$1,1,1$-Trichloroethane

1,1,2-Trichloroethane

1,1,2-Trichloroethane

1,1,2-Trichloroethane

1,1,2-Trichloroethane

1,1,2-Trichloroethane

1,1,2-Trichloroethane

- Trichloroethylene

Trichloroethylene

- Trichloroethylene

Trichloroethylene

Trichloroethylene

- Trichlorofluoromethane

Trichlorofluoromethane

Trichlorofluoromethane

Trichlorofluoromethane

Trichlorofluoromethane

Trichlorofluoromethane

Gross alpha

Gross alpha

Gross alpha

Gross alpha

Nonvolatile beta

Nonvolatile beta

Nonvolatile beta

Nonvolatile beta

Radium-226

Radium-226

Radium-226

Radium-226

Tritium

Tritium

Tritium

Tritium

\section{Result}

43

$<0.24$

$<0.24$

$<1.1$

$<1.1$

$<0.090$

$<0.090$

$<0.53$

$<0.54$

$<1.0$

22

24

22

24

21

$<1.0$

$<1.0$

$<5.0$

$<5.0$

$<5.0$

$<5.0$

4.9

4.7

5.3

4.8

4.8

$<1.0$

49

119

112

121

100

$2.0 E+00 \pm 4.0 E-01$

$<2.0 \mathrm{E}+00$

$<3 . \mathrm{OE}+00$

$<3.0 E+00$

$3.3 E+00 \pm 5.0 E-01$

$<2.0 E+00$

$<5.0 \mathrm{E}+00$

$5.5 E+00 \pm 2.7 E+00$

6.OE-O $1 \pm 1.0 \mathrm{E}-01$

6.OE-01 1 1.OE-O 1

5.1E-01 $\pm 6.9 \mathrm{E}-02$

6.3E-01 $\pm 7.9 \mathrm{E}-02$

1.7E + $00 \pm 4.0 \mathrm{E}-01$

2. $1 E+00 \pm 4.0 E-01$

$2.6 \mathrm{E}+00 \pm 4.5 \mathrm{E}-01$

$2.5 \mathrm{E}+00 \pm 4.5 \mathrm{E}-01$
Unit

Flag Lab

$\mu \mathrm{g} / \mathrm{L}$

$\mu g / L$

$\mu g / L$

$\mu g / L$

$\mu g / L$

$\mu g / L$

$\mu \mathrm{g} / \mathrm{L}$

$\mu \mathrm{g} / \mathrm{L}$

$\mu g / L$

$\mu g / L$

$\mu g / L$

$\mu g / L$

$\mu \mathrm{g} / \mathrm{L}$

$\mu g / L$

$\mu \mathrm{g} / \mathrm{L}$

$\mu g / L$

$\mu \mathrm{g} / \mathrm{L}$

$\mu g / L$

$\mu \mathrm{g} / \mathrm{L}$

$\mu g / L$

$\mu g / L$

$\mu \mathrm{g} / \mathrm{L}$

$\mu g / L$

$\mu \mathrm{g} / \mathrm{L}$

$\mu g / L$

$\mu g / L$

$\mu g / L$

$\mu g / L$

$\mu g / L$

$\mu g / L$

$\mu \mathrm{g} / \mathrm{L}$

$\mu \mathrm{g} / \mathrm{L}$

pCi/L

pCi/L

$\mathrm{pCi} / \mathrm{L}$

pCi/L

pCi/L

pCi/L

pCi/L

pCi/L

$\mathrm{pCi} / \mathrm{L}$

$\mathrm{pCi} / \mathrm{L}$

pCi/L

$\mathrm{pCi} / \mathrm{L}$

$\mathrm{pCi} / \mathrm{mL}$

$\mathrm{pCi} / \mathrm{mL}$

$\mathrm{pCi} / \mathrm{mL}$

$\mathrm{pCi} / \mathrm{mL}$

$\begin{array}{ll}0 & \text { WA } \\ 0 & \text { GE } \\ 0 & \text { GE } \\ 0 & \text { WA } \\ 0 & \text { WA } \\ 0 & \text { GE } \\ 0 & \text { GE } \\ 0 & \text { WA } \\ 0 & \text { WA } \\ 0 & \text { GE } \\ 0 & \text { GE } \\ 0 & \text { WA } \\ 0 & \text { WA } \\ 0 & \text { WA } \\ 0 & \text { WA } \\ 0 & \text { GE } \\ 0 & \text { GE } \\ 0 & \text { WA } \\ 0 & \text { WA } \\ 0 & \text { WA } \\ 0 & \text { WA } \\ 1 & \text { GE } \\ 1 & \text { GE } \\ 2 & \text { WA } \\ 1 & \text { WA } \\ 1 & \text { WA } \\ 0 & \text { GE } \\ 2 & \text { GE } \\ 2 & \text { WA } \\ 2 & \text { WA } \\ 2 & \text { WA } \\ 2 & \text { WA } \\ 0 & \text { GP } \\ 0 & \text { GP } \\ 0 & \text { CN } \\ 0 & \text { CN } \\ 0 & \text { GP } \\ 0 & \text { GP } \\ 0 & \text { CN } \\ 0 & \text { CN } \\ 0 & \text { TE } \\ 0 & \text { TE } \\ 0 & \text { CN } \\ 0 & \text { CN } \\ 0 & \text { GP } \\ 0 & \text { GP } \\ 0 & \text { CN } \\ 0 & \text { CN }\end{array}$

$\overline{0}$ = exceeded holding time.

= exceeded primary drinking water standard.

Sanitary Landfill 
WELL LFW 62B

\begin{tabular}{|c|c|c|c|c|c|}
\hline SRS Coord. & Lat/Longitude & Screen Zone Elevation & Top of Casing & Casing & Formation \\
\hline $\begin{array}{l}\text { N83001.2 } \\
\text { E45915.5 }\end{array}$ & $\begin{array}{l}33.284257^{\circ} \mathrm{N} \\
81.707124^{\circ} \mathrm{W}\end{array}$ & $72.8-62.8 \mathrm{ft} \mathrm{msl}$ & $164.9 \mathrm{ft} \mathrm{msl}$ & 4" PVC & \\
\hline
\end{tabular}

MEASUREMENTS CONDUCTED IN THE FIELD

Sample date: 06/05/92

Depth to water: $23.21 \mathrm{ft}(7.07 \mathrm{~m})$ below TOC Water elevation: $141.69 \mathrm{ft}(43.19 \mathrm{~m}) \mathrm{msl}$

Sp. conductance: $41 \mu \mathrm{S} / \mathrm{cm}$

Water evacuated before sampling: $208 \mathrm{gal}$

\section{LABORATORY ANALYSES}

Time: $12: 40$

pH: 4.7

Alkalinity: $0 \mathrm{mg} / \mathrm{L}$

Water temperature: $20.8^{\circ} \mathrm{C}$

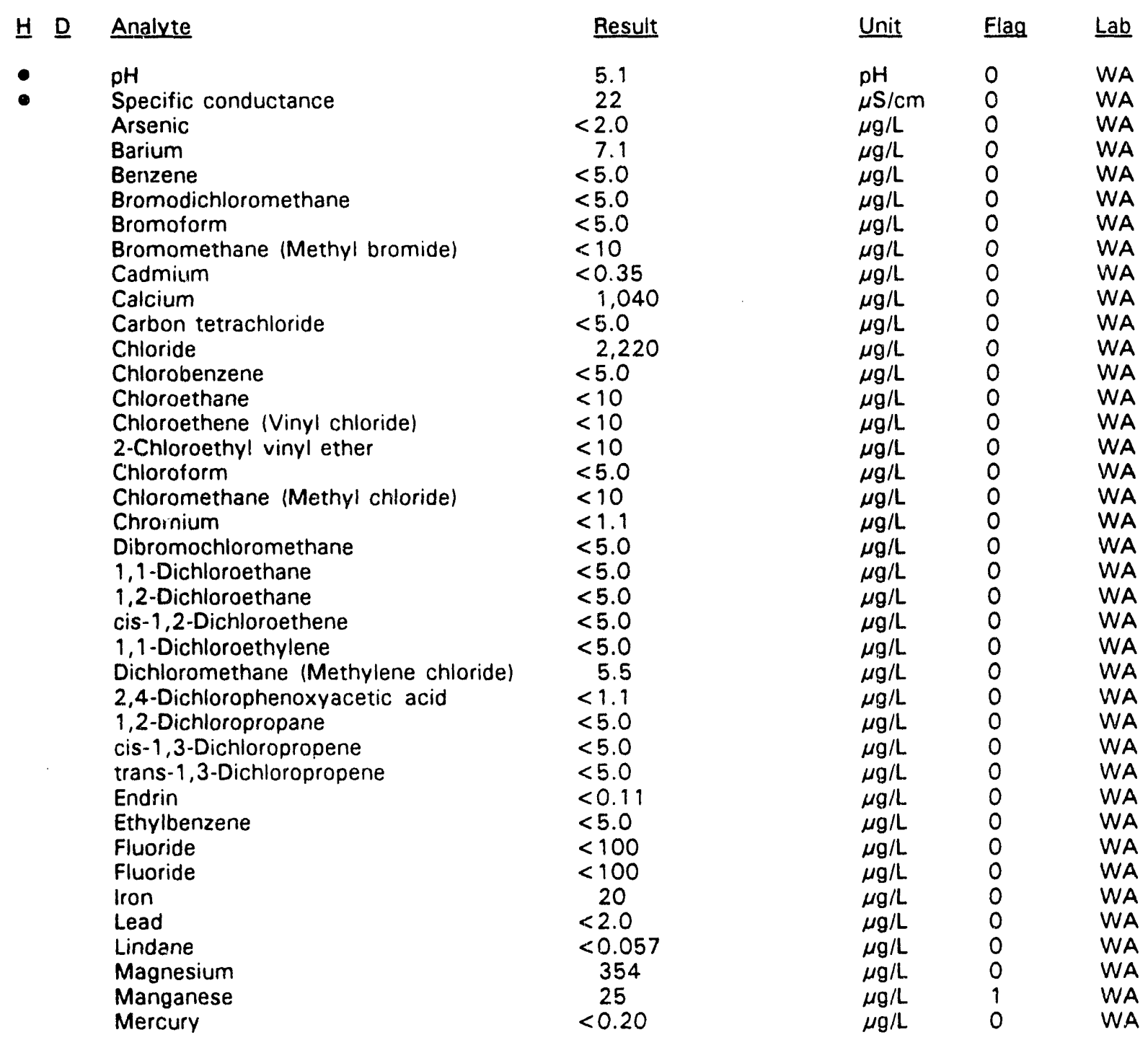

\footnotetext{
- = exceeded holding time. = exceeded primary drinking water standard.
} 
WELL LFW 62B collected on 06/05/92, laboratory analyses (cont.)

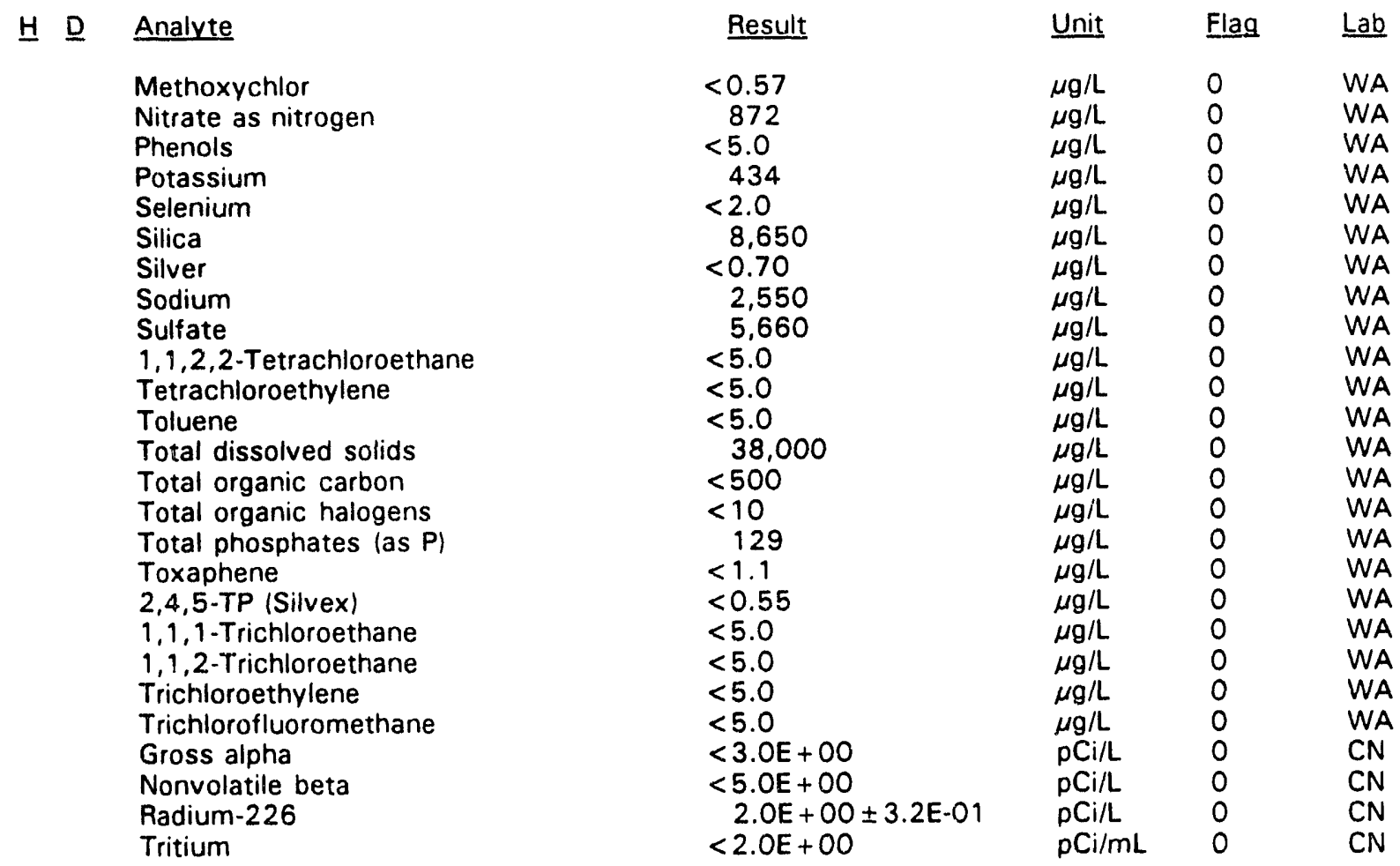

\section{WELL LFW 62C}

\begin{tabular}{|c|c|c|c|c|}
\hline SRS Coord. & Lat/Longitude & Screen Zone Elevation & Top of Casing & Casing \\
\hline $\begin{array}{l}\text { N83012.7 } \\
\text { E45906.7 }\end{array}$ & $\begin{array}{l}33.2842688^{\circ} \mathrm{N} \\
81.707169^{\circ} \mathrm{W}\end{array}$ & $118.4-108.4 \mathrm{ft} \mathrm{msl}$ & $165.5 \mathrm{ft} \mathrm{msl}$ & 4" PVC \\
\hline
\end{tabular}

\section{MEASUREMENTS CONDUCTED IN THE FIELD}

Sample date: 06/05/92

Depth to water: $23.43 \mathrm{ft}(7.14 \mathrm{~m})$ below TOC

Water elevation: $142.07 \mathrm{ft}(43.30 \mathrm{~m}) \mathrm{msl}$

Sp. conductance: $31 \mu \mathrm{S} / \mathrm{cm}$

Water evacuated before sampling: $89 \mathrm{gal}$

\section{LABORATORY ANALYSES}

$\begin{array}{ll}\text { - } D \quad \text { Analyte } & \text { Result } \\ \text { - } & \text { pH } \\ \text { Specific conductance } & 5.3 \\ \text { Arsenic } & 27 \\ \text { Barium } & <2.0 \\ \text { Benzene } & 7.2 \\ \text { Bromodichloromethane } & <5.0 \\ \text { Bromoform } & <5.0 \\ & <5.0\end{array}$

Time: $13: 10$

$\mathrm{pH}: 4.6$

Alkalinity: $0 \mathrm{mg} / \mathrm{L}$

Water temperature: $20.2^{\circ} \mathrm{C}$
WA
WA
WA
WA
WA
WA
WA
WA
WA
WA
WA
WA
WA
WA
WA
WA
WA
WA
WA
WA
WA
WA
CN
CN
CN
CN 
WELL LFW 62C collected on 06/05/92, laboratory analyses (cont.)

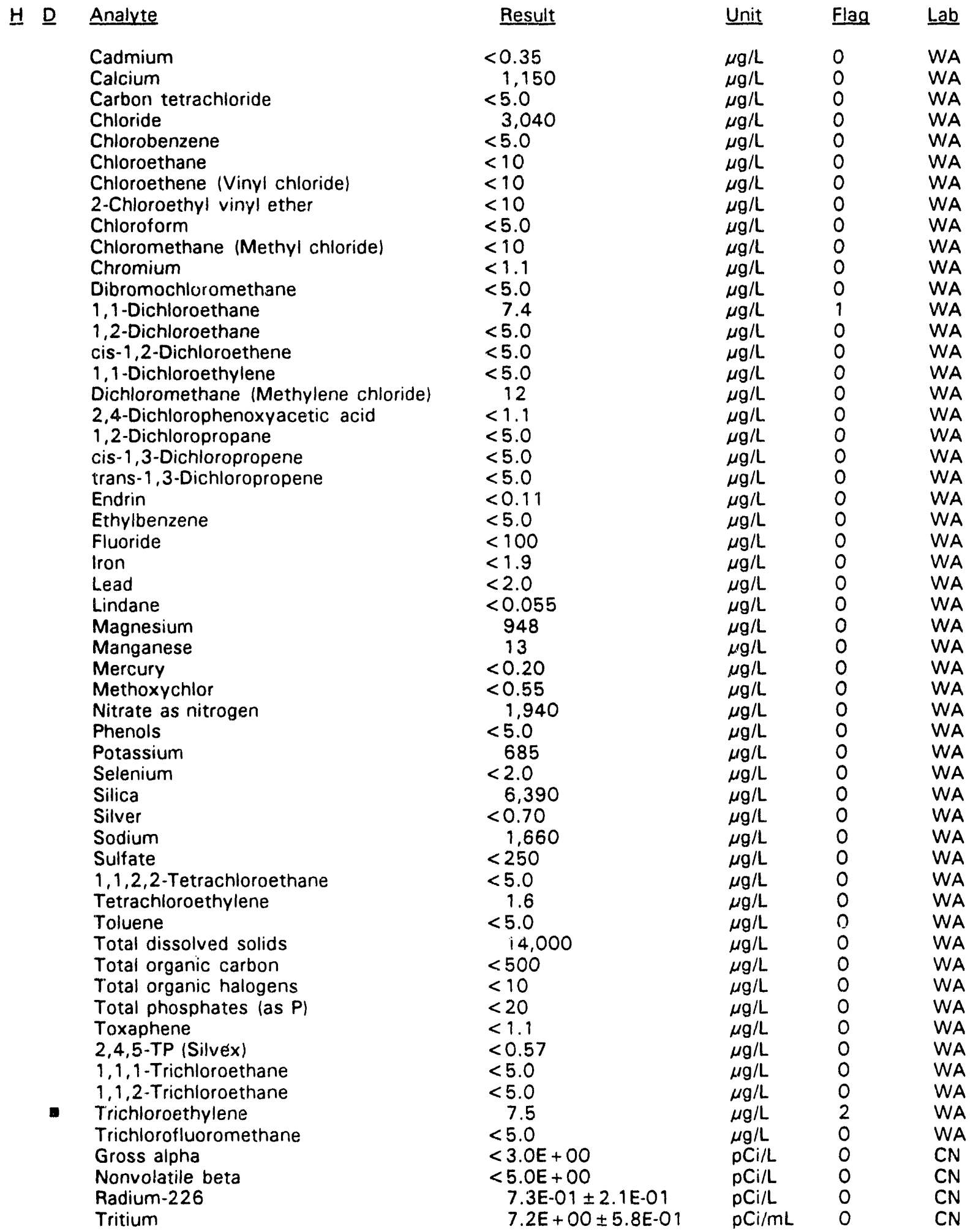

$\overline{-}=$ exceeded holding time. $=$ exceeded primary drinking water standard. 
WELL LFW 62D

$\begin{array}{llllll}\text { SRS Coord. } & \text { Lat/Longitude } & \text { Screen Zone Elevation } & \text { Top of Casing } & \text { Casing } & \text { Formation } \\ \text { N82991.6 } & 33.284247^{\circ} \mathrm{N} & 147.6-127.6 \mathrm{ft} \mathrm{msl} & 164.8 \mathrm{ft} \mathrm{msl} & 4^{\text {" PVC }} \\ \text { E45922.9 } & 81.707086^{\circ} \mathrm{W} & & & \end{array}$

\section{MEASUREMENTS CONDUCTED IN THE FIELD}

Sample date: 06/05/92

Depth to water: $22.51 \mathrm{ft}(6.86 \mathrm{~m})$ below TOC

Water elevation: $142.29 \mathrm{ft}(43.37 \mathrm{~m}) \mathrm{msl}$

Sp. conductance: $39 \mu \mathrm{S} / \mathrm{cm}$

Water evacuated before sampling: 10 gai

The well went dry during purging.

\section{LABORATORY ANALYSES}

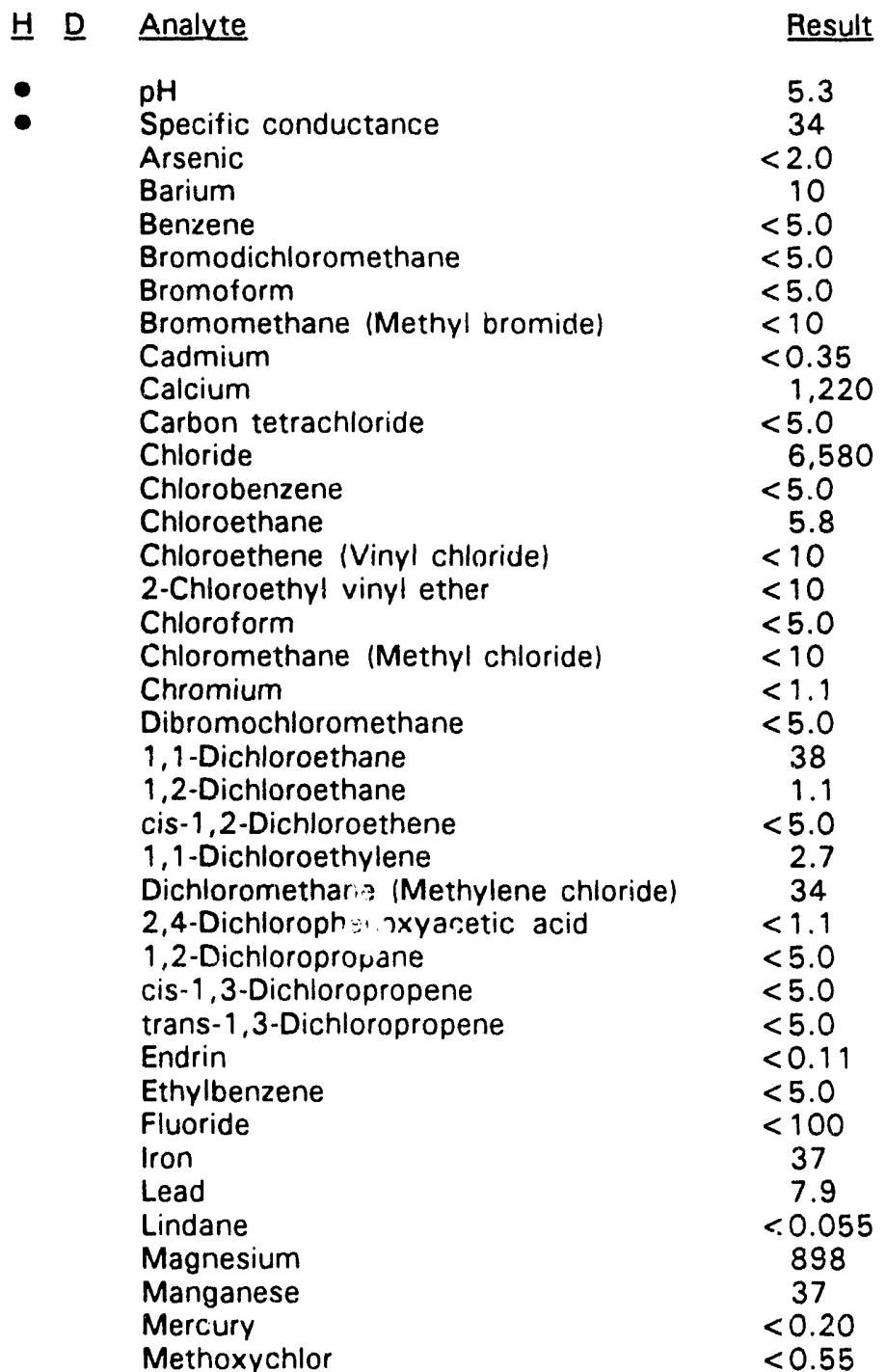

Time: $12: 20$

$\mathrm{pH}: 3.8$

Alkalinity: $1 \mathrm{mg} / \mathrm{L}$

Water temperature: $19.8^{\circ} \mathrm{C}$ 
WELL LFW 62D collected on 06/05/92, laboratory analyses (cont.)

$\begin{array}{ll}\text { H. } & \text { Analyte } \\ \text { Nitrate as nitrogen } \\ \text { Phenols } \\ \text { Potassium } \\ \text { Selenium } \\ \text { Silica } \\ \text { Silver } \\ \text { Sodium } \\ \text { Sulfate } \\ \text { 1,1,2,2-Tetrachloroethane } \\ \text { Tetrachloroethylene } \\ \text { Toluene } \\ \text { Total dissolved solids } \\ \text { Total organic carbon } \\ \text { Total organic halogens } \\ \text { Total phosphates (as P) } \\ \text { Toxaphene } \\ \text { 2,4,5-TP (Silvex) } \\ \text { 1,1,1-Trichloroethane } \\ \text { 1,1,2-Trichloroethane } \\ \text { Trichloroethylene } \\ \text { Trichlorofluoromethane } \\ \text { Gross alpha } \\ \text { Nonvolatile beta } \\ \text { Radium-226 } \\ \text { Tritium } \\ \text { Tritium }\end{array}$

$\begin{aligned} & \text { Result } \\ & 367 \\ &<5.0 \\ & 603 \\ &<2.0 \\ & 8.710 \\ & 1.2 \\ & 3,560 \\ & 529 \\ &<5.0 \\ & 3.1 \\ &<5.0 \\ & 35,000 \\ & 865 \\ & 202 \\ & 123 \\ &<1.1 \\ &<0.54 \\ & 26 \\ &<5.0 \\ & 11 \\ & 89 \\ &<3.0 E+00 \\ &<5.0 E+00 \\ & 6.7 E-01 \pm 2.4 E-01 \\ & 5.0 E+00 \pm 5.0 E-01 \\ & 4.4 E+00 \pm 4.8 E-01\end{aligned}$

$\begin{array}{llll}\text { Unit } & \text { Flag } & \text { Lab } \\ \mu \mathrm{g} / \mathrm{L} & 0 & \text { WA } \\ \mu \mathrm{g} / \mathrm{L} & 0 & \text { WA } \\ \mu \mathrm{g} / \mathrm{L} & 0 & \text { WA } \\ \mu \mathrm{g} / \mathrm{L} & 0 & \text { WA } \\ \mu \mathrm{g} / \mathrm{L} & 0 & \text { WA } \\ \mu \mathrm{g} / \mathrm{L} & 0 & \text { WA } \\ \mu \mathrm{g} / \mathrm{L} & 0 & \text { WA } \\ \mu \mathrm{g} / \mathrm{L} & 0 & \text { WA } \\ \mu \mathrm{g} / \mathrm{L} & 0 & \text { WA } \\ \mu \mathrm{g} / \mathrm{L} & 1 & \text { WA } \\ \mu \mathrm{g} / \mathrm{L} & 0 & \text { WA } \\ \mu \mathrm{g} / \mathrm{L} & 0 & \text { WA } \\ \mu \mathrm{g} / \mathrm{L} & 0 & \text { WA } \\ \mu \mathrm{g} / \mathrm{L} & 2 & \text { WA } \\ \mu \mathrm{g} / \mathrm{L} & 0 & \text { WA } \\ \mu \mathrm{g} / \mathrm{L} & 0 & \text { WA } \\ \mu \mathrm{g} / \mathrm{L} & 0 & \text { WA } \\ \mu \mathrm{g} / \mathrm{L} & 0 & \text { WA } \\ \mu \mathrm{g} / \mathrm{L} & 0 & \text { WA } \\ \mu \mathrm{g} / \mathrm{L} & 2 & \text { WA } \\ \mu \mathrm{g} / \mathrm{L} & 2 & \text { WA } \\ \mathrm{pCi} / \mathrm{L} & 0 & \text { CN } \\ \mathrm{pCi} / \mathrm{L} & 0 & \text { CN } \\ \mathrm{pCi} / \mathrm{L} & 0 & \text { CN } \\ \mathrm{pCi} / \mathrm{mL} & 0 & \text { CN } \\ \mathrm{pCi} / \mathrm{mL} & 0 & \text { CN }\end{array}$

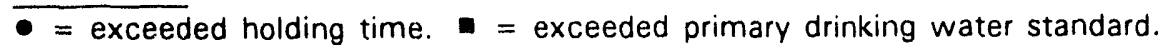



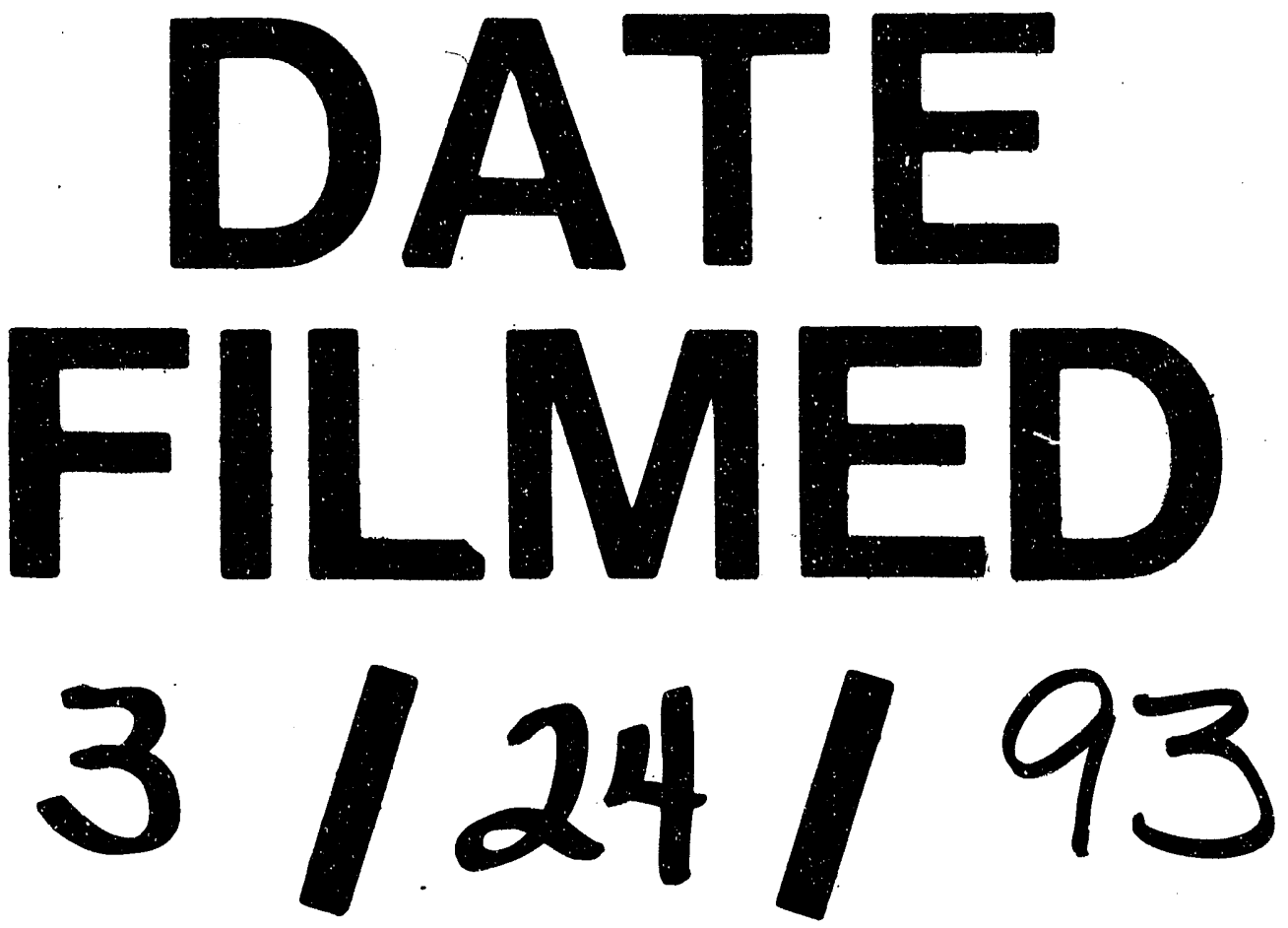